\title{
Filtering of species specific song parameters via interneurons in a bush cricket's brain
}

\author{
DISSERTATION \\ for the award of the degree \\ "Doctor rerum naturalium" (Dr. rer. nat.) \\ Division of Mathematics and Natural Sciences \\ of the Georg-August-Universität Göttingen
}

\author{
submitted by \\ Tim Daniel Ostrowski \\ from Leer (Loga)
}

Göttingen 2009 


\section{Members of the Thesis Committee}

Prof. Dr. Andreas Stumpner (first reviewer)

Department of Neurobiology, Göttingen University

Prof. Dr. Norbert Elsner (second reviewer)

Department of Neurobiology, Göttingen University

Prof. Dr. Tobias Moser

Department of Otolaryngology, Göttingen University Medical School

Date of the oral examination: 17.12.2009 


\section{Declaration}

I hereby declare that the thesis:

„Filtering of species specific song parameters via interneurons in a bush cricket's brain"

has been written independently and with no other sources and aids than quoted.

Tim Daniel Ostrowski 



\section{Table of contents}

1 INTRODUCTION

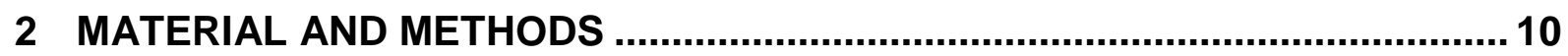

2.1 Laboratory breeding of Ancistrura nigrovittata...................................... 10

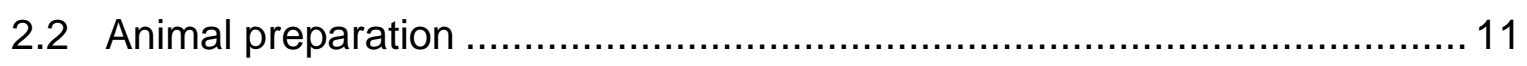

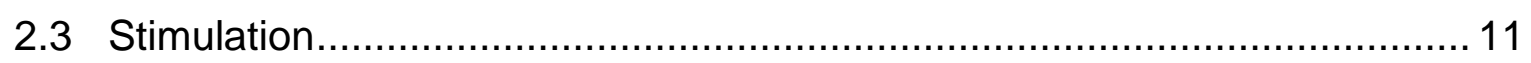

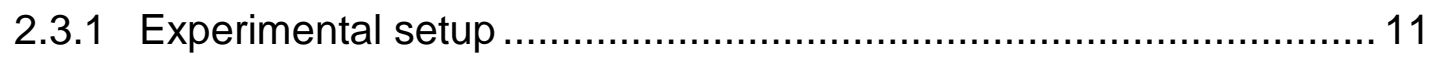

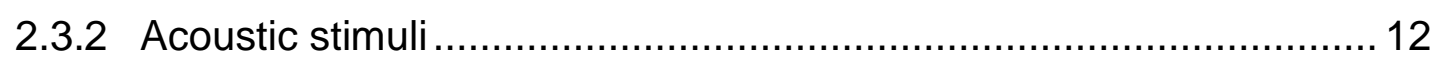

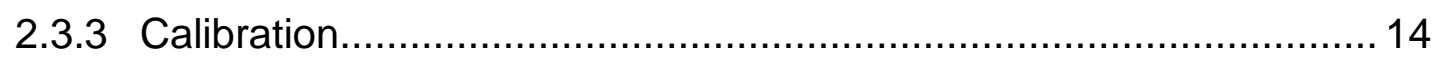

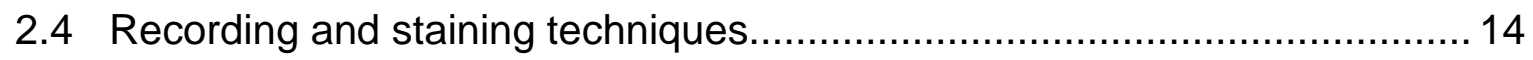

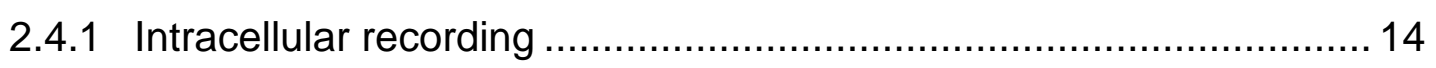

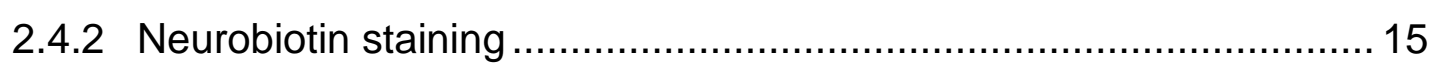

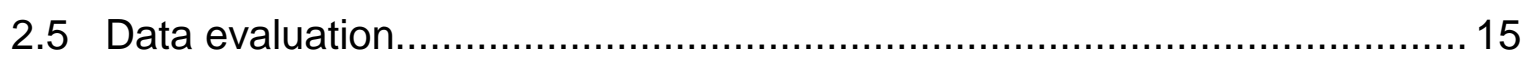

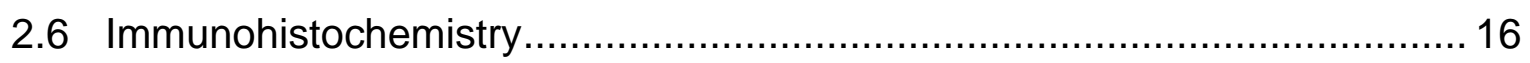

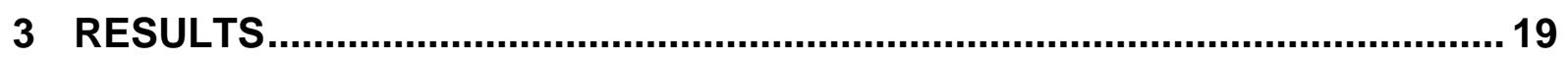

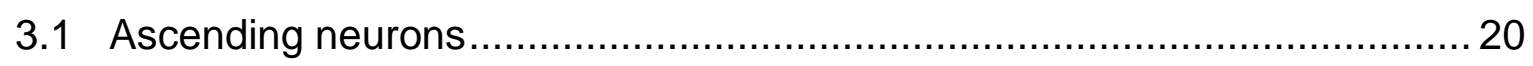

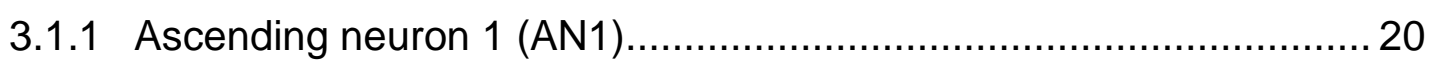

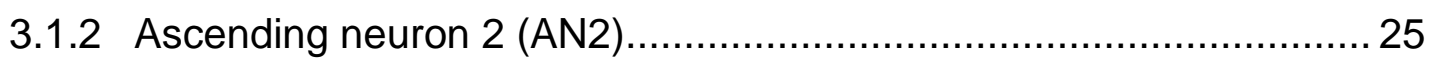

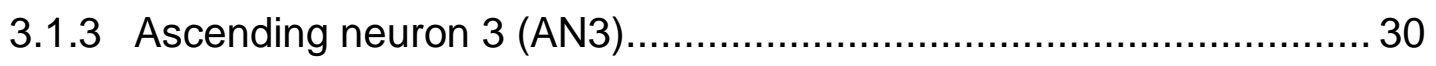

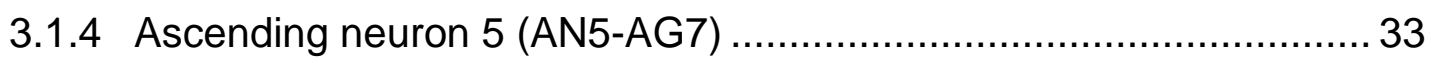

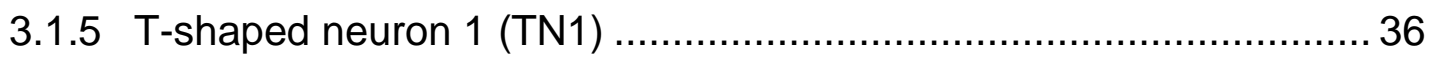

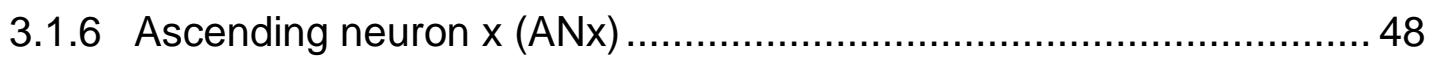

3.1.7 Ascending vibratory neurons ................................................... 51

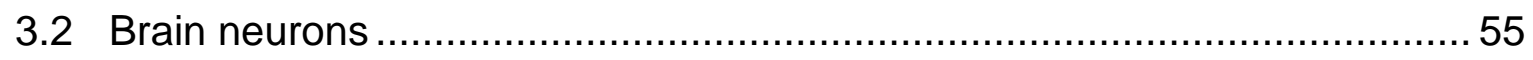

3.2.1 Local brain neuron 1 (LBN1) .................................................... 55

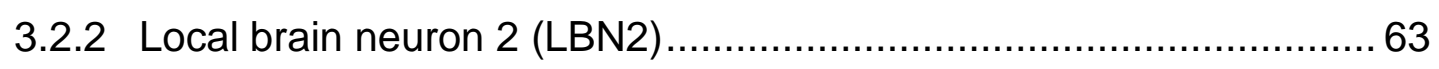


3.2.3 Local brain neuron 3 (LBN3) …….......................................... 70

3.2.4 Local brain neuron 4 (LBN4) ………........................................

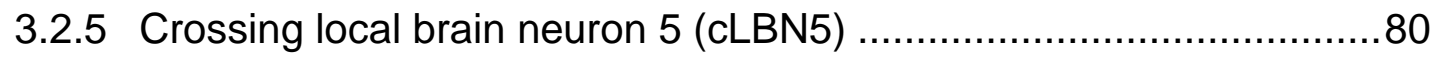

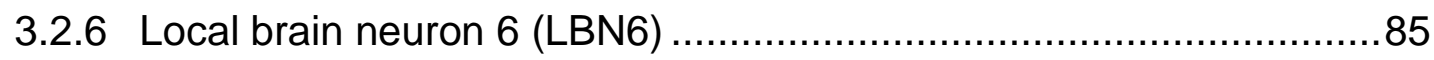

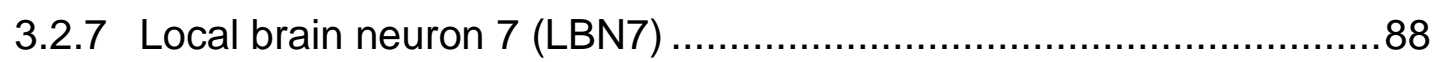

3.2.8 Crossing local brain neuron 8 (cLBN8) .......................................95

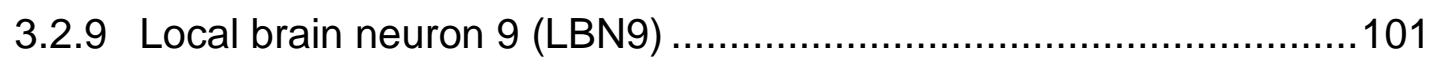

3.2.10 Local brain neuron 10 (LBN10) ................................................ 105

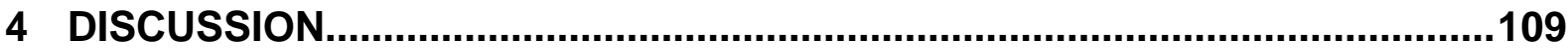

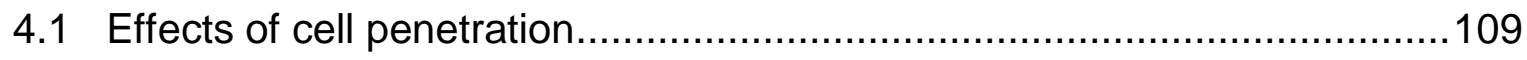

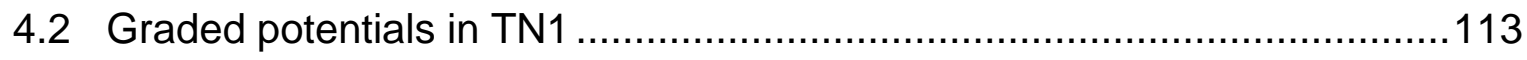

4.3 Possible function of the identified auditory brain neurons .........................116

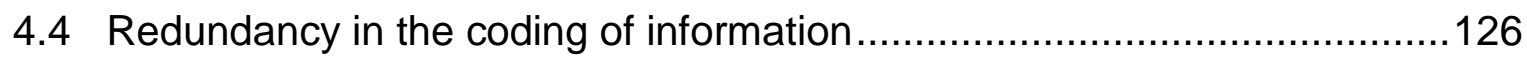

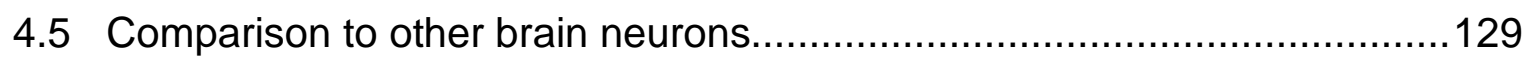

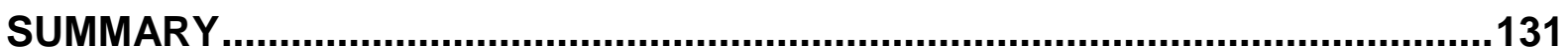

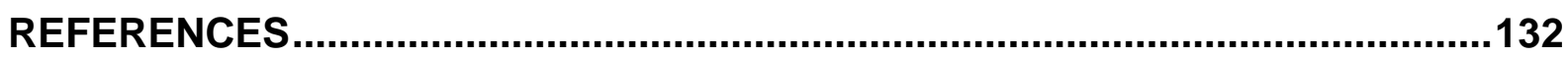

APPENDIX

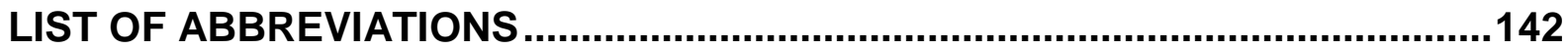

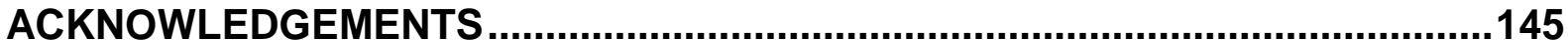

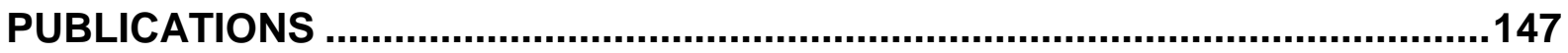

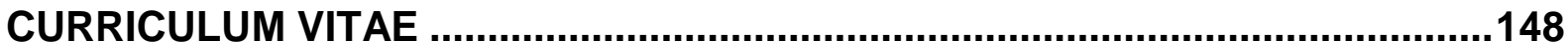




\section{Introduction}

Intraspecific communication within various animal species has been the subject of research since many years (e.g. Bradbury \& Vehrencamp 1998). Beyond the descriptive analysis of behaviour one tries to understand the neuronal mechanisms that cause the complexity of social interactions. These mechanisms may be processes of recognition, identification and the release of an appropriate response. In order to identify the function of the underlying neuronal networks, a model system with a reduced complexity can be used that exhibits inherited and stereotyped behaviour. Additionally, the system needs to be easily accessible and a behavioural response should clearly define the parameters of recognition. The acoustic communication in insects may serve as such a model. Due to the conserved principles of information processing within nervous networks one may transfer some of the findings obtained in "simple" networks for a better understanding of complex ones.

Acoustic communication in orthoptera. The research in acoustic communication has experienced special attention in the context of pair formation and courtship in orthopterans (e.g. Alexander 1967; Ewing 1984; Gerhardt \& Huber 2002). In ensiferans, airborne signals are generated by rubbing both forewings against each other (Simmons 1988; Heller 1990). Male crickets produce a calling song that causes positive phonotaxis in the mute conspecific female and thus, drastically increases the probability of encounter (Otte 1992). In many singing grasshopper species, the song of the male is answered by the female, which then results in mutual positive phonotaxis (Helversen \& Helversen 1983). This can also be observed in certain bush crickets species where, for example, males produce a more or less complex calling song that is answered by the female with a single short click (Heller 1990; Stumpner \& Meyer 2001; Tauber et al. 2001; review: Bailey 2003). However, acoustic signals with transmission of the signal through the environment to the receiver - often involves (i) the danger of being detected by predators (Cade 1984; Sakaluk \& Belwood 1984), (ii) a potentially low signal to noise ratio for long distance calls (Michelsen 1978; Forrest 1994), (iii) attraction of heterospecific females possibly leading to hybridisation (Harrison 1986; Vedenina \& Helversen 2003), while its main function is to ensure a correct "understanding" by the conspecific female (Stabel et al. 1989; Doherty 1991). The latter is achieved by a close behavioural tuning of the 
partner to the species specific song that leads to a positive phonotactic response. The identification of potential predators, on the other hand, should elicit the opposite behaviour (Moiseff et al. 1978; Nolan \& Hoy 1986). The song of a species clearly defines the singer as conspecific and may also transmit secondary informations as, for example, the constitutional health of the singer (e.g. Ryder \& Siva-Jothy 2000). Nevertheless, "understanding" the song structure and identification as being conspecific requires a neuronal network that is capable of extracting the species specific parameters. Many behavioural and neurophysiological studies have been performed in orthopteran species to shed light on the potential mechanisms behind the recognition process (Pollack \& Hoy 1979; Schildberger 1984a; Schul 1998), whereby decisive steps of pattern recognition seem to be made within the brain (Ronacher et al. 1986; Bauer \& Helversen 1987, Pires \& Hoy 1992).

A system that has the vantage of being relatively easy accessible and where the parameters of song recognition are clearly defined, is found in the duetting bush cricket Ancistrura nigrovittata (Brunner von Wattenwyl, 1878). Extensive knowledge about the behavioural responses and neuronal processing of auditory information on the first level of the central nervous system (CNS) has been gathered in the last 20 years (Heller \& Helversen 1986; Dobler et al. 1994a, b; Stumpner 1997, 1999a; Stumpner \& Molina 2006). Completely missing, so far, is knowledge about processing of auditory information in the brain - the location where most likely the final steps of recognition take place.

Behaviour of Ancistrura nigrovittata. For mate finding males of the southerneuropean bush cricket Ancistrura nigrovittata (Phaneropterinae, Barbitistini) produce a calling song to which motivated conspecific females respond (Figure 1). An analysis of the relevant temporal and spectral parameters of the duet was the main subject in the work of Dobler et al. (1994a, b) and partly of Heller \& Helversen (1986). With the detailed knowledge about the important elements in the song of $A$. nigrovittata for the phonotactic behaviour a comparison to the activity of auditory neurons can be made.

The male calling song comprises a sequence of verses whereby each verse consists of a series of 5 to 9 syllables ("syllable group"; pulse/pause duration: approximately $7 / 22 \mathrm{~ms}$ ) and a single syllable - the so called "trigger syllable" - that follows the syllable group with a constant latency of ca. $400 \mathrm{~ms}$. Both elements of the male song were thoroughly tested by variations of the temporal structure in behavioural experiments with female A. nigrovittata (Dobler et al. 1994a). A 
presentation of an artificial song elicited the highest responses of females at the natural values. The syllable group, for example, which has to contain at least 5 syllables, plays a particularly important role in the recognition process. Models of the male calling song with increased or decreased pulse durations cause a successively reduced response in females. The same holds true for variations of the pause duration between pulses. The final trigger syllable, on the other hand, plays a crucial role in the release of the female answer. Highest behavioural responses were found at natural values only, when the latency of the trigger pulse to the syllable group was varied. The response of the female, however, consists of a short tick (ca. $1 \mathrm{~ms}$ ) that follows the onset of the trigger syllable with a constant latency of about $35 \mathrm{~ms}$ (Heller \& Helversen 1986). The latency of the female reply to the male trigger pulse seems to be important for the male's recognition process. It has been shown that males possess a certain time-window, in which the female reply has to occur to be recognised by the male (Heller \& Helversen 1986; Zimmermann et al. 1989). Preliminary data of behavioural tests with male A. nigrovittata also suggests the existence of such a time-window. If the latency of the female reply exceeds approximately $70 \mathrm{~ms}$, the number of male responses decrease (Pierre Gras personal communication).

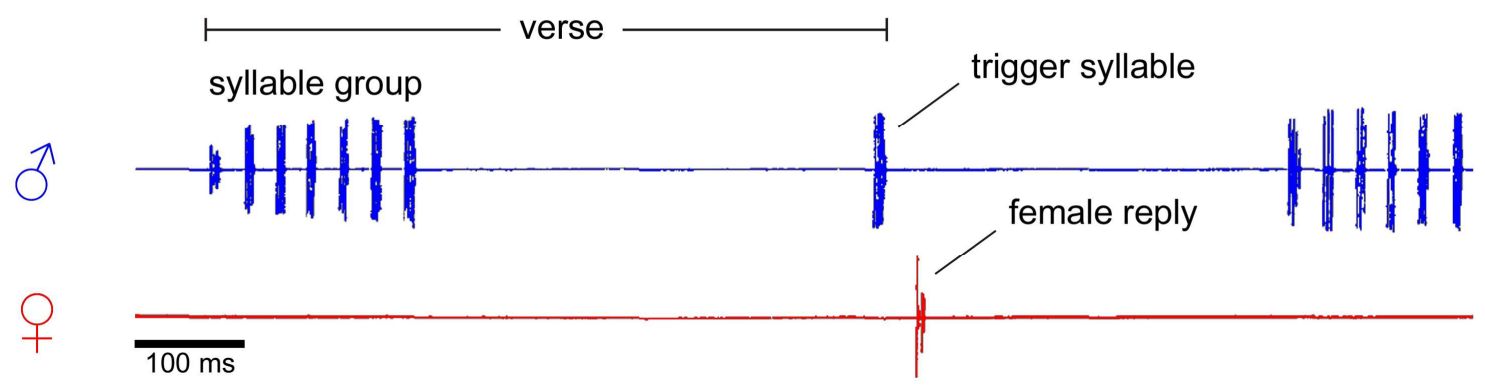

Figure 1: Oscillogram of the male song (blue) and the female reply (red) in A. nigrovittata (modified after Dobler et al. 1994a).

Besides the temporal composition, the duet of A. nigrovittata, furthermore, comprises a sex specific carrier frequency difference which is uncommon among other duetting bush crickets that have been examined (Nickle 1976; Heller \& Helversen 1986; Stumpner \& Meyer 2001). The male calling song has its maximum energy peaking between $12-16 \mathrm{kHz}$, whereas the female reply's energy peaks between $28-30 \mathrm{kHz}$. This difference in song spectra serves as a parameter (in addition to the temporal structure) for recognition of the opposite sex in this species. Behavioural responses of males and females clearly showed a narrow tuning to the carrier frequency of the opposite song (Dobler et al. 1994b). For the female 
behaviour to artificial male calls, also a dependence on intensity was determined. The best response activity was found at approximately $20 \mathrm{~dB}$ above the behavioural threshold that decreases with a further increase of intensity (Dobler et al. 1994b). Non-invasive behavioural experiments can, therefore, reveal the decisive parameters of a species specific song and may also allow hypothesis about certain underlying neuronal mechanisms (Helversen \& Helversen 1995; Helversen \& Helversen 1998; Bush \& Schul 2006). A more precise identification of the neuronal mechanisms, however, can be achieved with intracellular recordings of the participating nerve cells.

Sound gathering apparatus of $A$. nigrovittata. Is behavioural tuning to the song of the conspecific partner just a passive by-product of the sound gathering structures? In general, these structures do partly contribute to the process of recognition due to their biophysical properties. A fundamental discovery for the mammalian ear was the spatial mapping of carrier frequencies along the basilar membrane, which is also known as "tonotopy" (Békésy 1960). This tonotopy bases on a sound evoked displacement of the basilar membrane at a carrier frequency specific area. Thus, a discrimination of different carrier frequencies is made possible and the filtered information can then be transmitted via afferent nerve cells (sensory neurons) to the central nervous system (for a review see: Robles \& Ruggero 2001).

Also in hearing insects such a tonotopy - in some aspects strikingly similar to mammals - can be found (e.g. Michelsen 1971; Oldfield 1982; Oldfield et al. 1986; Stölting \& Stumpner 1998). The crista acustica of $A$. nigrovittata comprises about 35 to 37 receptor cells that are aligned along parts of the acoustic trachea. This trachea forwards most of the airborne sound from the spiracle to the tympanum, which is situated near the "knee" of the foreleg (Heinrich et al. 1993; Michelsen et al. 1994; Stumpner 1996 - Figure 2 left). In the crista acustica the size of the cap cells and scolopidia gradually decrease in distal direction, alongside with the tuning to higher carrier frequencies of the primary afferents (Schwabe 1906, Oldfield 1982; Lin et al. 1993). Thus, each receptor represents a more or less narrow frequency band (Figure 2 right), whereas a dependence of spike activity on temporal patterns could not be shown (Schul 1997). The compartmentalised information about carrier frequency can then be forwarded via the auditory nerve to the CNS (Römer 1983). 
crista acustica

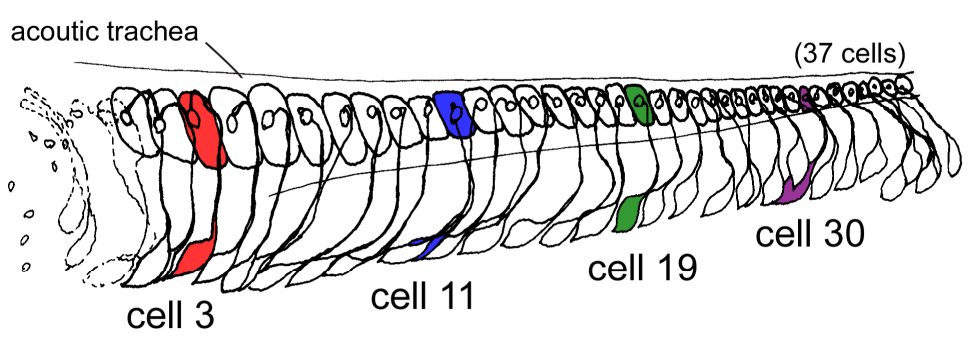

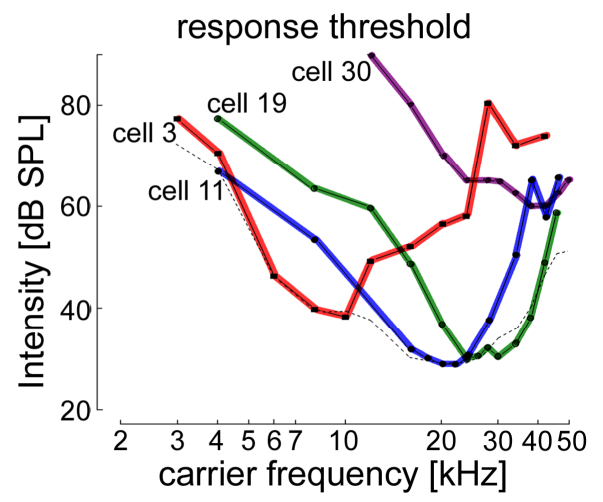

Figure 2: Left: Crista acustica of $A$. nigrovittata. Right: Frequency tuning curves of the receptor cells that are marked correspondingly in the crista acustica (left). Dotted line indicates the overall hearing threshold. Modified after Stumpner (2001).

Central neuronal organisation of A. nigrovittata. At what point of the specimen's nervous system may the mechanisms, responsible for the recognition of the conspecific partner, be situated? First of all, tonotopic organisation of carrier frequency can also be found in the auditory neuropil of the prothoracic ganglion - the "first station" of central auditory processing (Oldfield 1983; Römer 1983; Stumpner 1996). Here, the acoustic information of afferents is picked up by auditory interneurons that exhibit manifold physiological and morphological characteristics (e.g. Römer 1987; Römer et al. 1988; Stumpner 2001; Stumpner \& Helversen 2001; Stumpner \& Molina 2006).

The omega neuron, the hemiganglionic cells (SN) and some dorsal-unpairedmedian (DUM) neurons are local auditory interneurons in A. nigrovittata (Stumpner 2001; Molina \& Stumpner 2005). DUM neurons are described in numerous studies, whereby most of them do not respond to sound (Gras et al. 1990). Little information is available about DUM neurons that are activated by sound. They seem to have a direct synaptic connection to afferents (but additionally receive frequency specific inhibition). Their main function is thought to be the inhibition of other auditory interneurons (Stumpner 2001, 2002; Stritih \& Stumpner 2009; personal communication: A. Stumpner). At least two SN's can be found in A. nigrovittata with a broad frequency tuning. Their potential contribution to song recognition, though, is completely obscure (Stumpner 2001). Many studies concentrated on the physiology and morphology of the omega neuron (ON1) (in crickets: Popov et al. 1978; Atkins \& Pollack 1986; in bush crickets: Römer \& Krusch 2000; Molina \& Stumpner 2005), which obviously is homologous in bush crickets and crickets (Zhantiev and Korsunovskaya 1983). For crickets also an additional omega neuron (ON2) is known that has not been found in bush crickets (Wohlers \& Huber 1982; Watson \& Hardt 
1996). The main function of the broadly tuned ON1 is seen in the inhibition of contralateral neurons and therefore, to play a major role in the processing of directional cues (Selverston et al. 1985; Stumpner et al. 1995; Römer \& Krusch 2000), which has also been demonstrated for $A$. nigrovittata (Molina \& Stumpner 2005). Furthermore, in crickets a participation in the analysis of temporal parameters is suggested by a few authors (Wiese \& Eilts 1985; Nabatiyan et al. 2003). Certainly, not all behavioural responses regarding the localisation and recognition may be explained by response properties of the local omega neuron alone (Atkins et al. 1984).

Other interneuron types possess dendritic arborisations in the prothorax and an axon that descends to the abdominal ganglia or an axon that ascends to the brain or both (Stumpner 1995; Stumpner \& Molina 2006). In A. nigrovittata some descending neurons (DNs) are known, that seem to be broadly tuned but most are responsive to vibrations. The potential contribution in the processing of conspecific signals is unknown (Stumpner 2001; Stritih \& Stumpner 2009). Descending neurons have also been described for crickets with similar physiological properties (Wohlers \& Huber 1982; Atkins \& Pollack 1987a, b). Two interneurons are known in A. nigrovittata, namely T-shaped neuron 1 and 3 (TN1 and TN3), that have two axons - one descending to the abdominal ganglia and one ascending to the brain (Stumpner 1999a, 2001). TN3 may only play a role in thoracic processing, since it produces strong excitatory postsynaptic potentials (EPSPs) whereas only occasionally spikes are elicited (Stumpner \& Molina 2006). TN1, on the other hand, may play a profound role in the processing of conspecific signals, since its spiking thresholds especially for ultrasonic frequencies are low (for carrier frequencies above $20 \mathrm{kHz}$ close to the minimal threshold obtained from various primary afferents) (Stumpner 1999a; Stumpner \& Molina 2006). TN1 responds well to the complete duet (with a strong coupling to the temporal pattern), whereas strongest responses are found to the female answer (Stumpner \& Molina 2006). Additionally to a descending axon, TN1 exhibits an axon ascending to the auditory neuropil of the brain with dense arborisations indicating its main region of axonal output (Figure 3). Putative homologues were found in several ensiferan species (Wohlers \& Huber 1985; Römer et al. 1988; Stritih \& Stumpner 2009; Triblehorn \& Schul 2009). The interneuron AN5AG7 (also referred to as "AN5") of $A$. nigrovittata has a unique morphology among the auditory neurons of bush crickets and crickets, since no homologues were found so far (Stumpner 1999a). The soma of AN5 is located in the seventh abdominal ganglion, whereas its major dendritic input is situated in the auditory neuropil of the 
prothorax closely overlapping with afferents that are tuned to ultrasonic frequencies (Figure 3). The axonal output of AN5 is found in the auditory neuropil in the lateral protocerebrum of the brain. Its spiking response is highest to the female song frequency (ca. $28 \mathrm{kHz}$ ), which mirrors the overlap with the receptors. In addition, AN5 receives strong inhibition at frequencies below $24 \mathrm{kHz}$. These findings strongly suggest AN5 to be involved in the analysis of the female reply (Stumpner 1999b).

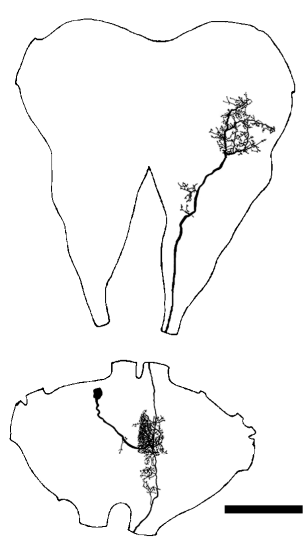

TN1
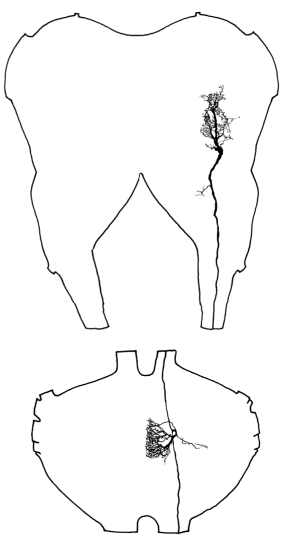

AN5-AG7
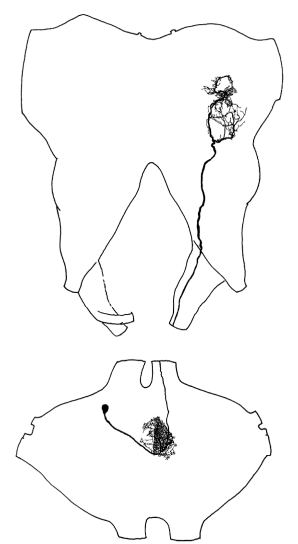

AN1
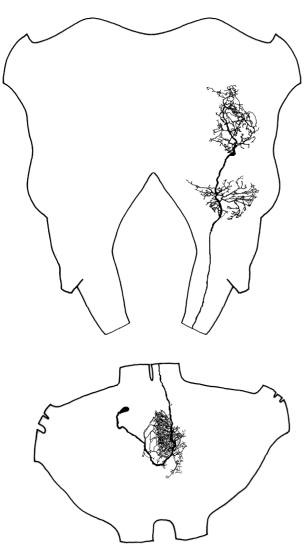

AN2
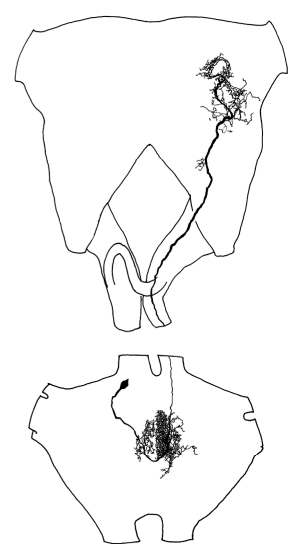

AN3

Figure 3: Morphology of all auditory interneurons described so far that possess an axon ascending in the lateral protocerebrum of the brain of A. nigrovittata (except AN4). Bar represents $200 \mu \mathrm{m}$. Drawings from A. Stumpner (AN2) and modified after Stumpner 1997 (AN1), 1999a (TN1 and AN5AG7), Stumpner \& Molina 2006 (AN3).

Auditory interneurons in A. nigrovittata that exhibit an axon ascending to the brain only, are ascending neuron 1, 2, 3 and 4 (AN1-4) (Stumpner \& Molina 2006). Among these neurons, AN1 (Figure 3) exhibits response characteristics that suggest a critical involvement in male song processing (Stumpner 1997). With highest spike activity between 12 and $16 \mathrm{kHz}$ it is narrowly tuned to the male song frequency. This tuning is, to a minor degree, due to an overlap with primary afferents that process a similar frequency range. The main factor for frequency selectivity, however, is the inhibition that AN1 receives at carrier frequencies below and above $15 \mathrm{kHz}$. This selectivity of frequency vanishes mainly (but not exclusively) when picrotoxin, a $\gamma$-aminobuturic acid-A $\left(\mathrm{GABA}_{\mathrm{A}}\right)$ channel blocker, is applied (Stumpner 1998) (a substance known to remove all inhibition in the invertebrate CNS - Robbins \& van der Kloot 1958). Then, AN1's tuning is as broadly banded as found in the omega neuron (Stumpner 2002). Little dependence on temporal pattern is found in the response of AN1, although a good coupling to the male calling song is found (Stumpner 1997; Stumpner \& Molina 2006). AN2 is an ascending neuron that exhibits, additionally to the common arborisation sites, dense presumably postsynaptic ramifications in the 
deutocerebrum of the brain (Figure 3). Therefore, it is most likely that AN2 receives a secondary input (besides the auditory input in the prothoracic ganglion) in the brain that cannot be recorded in the thorax. Thoracic recordings revealed a lowest threshold of AN2's response for frequencies close to the male song frequency (ca. $14-16 \mathrm{kHz}$ - not as low as found for AN1) and strong frequency dependent inhibition (Stumpner 2001; Stumpner \& Molina 2006). The third ascending neuron in A. nigrovittata, namely AN3, exhibits tonic response characteristics (similar as found for receptors) to all stimulus pattern for a broad range of frequencies. AN3 is discussed to function as a "reference channel" for other neurons that signals the arrival of airborne sound with no specific selectivity to carrier frequency or temporal pattern. Its morphology is similar to that of AN1 (Figure 3), although more dendritic ramifications are found on the soma-ipsilateral side, which is reflected in a bilateral excitation of AN3 (Stumpner \& Molina 2006). The response of AN3, therefore, shows only little directional dependence. AN4 has lowest response thresholds between 20 and $35 \mathrm{kHz}$ ( $50 \mathrm{~dB} \mathrm{SPL})$ and can be clearly distinguished from other ascending neurons by its morphology (not displayed in Figure 3, but see Stumpner \& Molina 2006). Its soma is located posteriorly and only few arborisations are found in the auditory neuropil in the lateral protocerebrum of the brain (Stumpner 2001; Stumpner \& Molina 2006). For most of the ascending neurons described above, also homologues were found in other bush crickets (Römer et al. 1988; Sickmann 1996; Stumpner 2002) as well as in crickets (Wohlers \& Huber 1982; Hennig 1988).

The data obtained from thoracic recordings in the past reveal filter properties of auditory neurons that show correlations to some, but not all of the behavioural responses found in A. nigrovittata. Therefore, a filtering of species specific song parameters, which shows a more closely correlation to the behaviour, can be expected on subsequent levels of auditory processing.

No auditory interneuron has been identified in the brain of any bush cricket so far. In Ancistrura nigrovittata nothing is known about the processing of conspecific signals in the brain. Thus, the present work will concentrate on the "second station" of auditory processing in the CNS of A. nigrovittata. The following major questions will be addressed:

1. What is the information arriving in the brain? Can the response properties of ascending neurons, described from thoracic recordings in the dendritic area, be verified when the recordings are performed from the axonal terminals in the 
brain? Are there more ascending neurons, not described from thoracic recordings so far?

2. What types of local auditory neurons are found in the brain of $A$. nigrovittata? Are they obvious homologous to brain neurons described in crickets?

3. What types of auditory processing are found in the brain with special emphasis on filtering of carrier frequency of male song and female reply and on conspecific temporal parameters? Are there mechanisms of processing not identified in the thorax? 


\section{Material and Methods}

\subsection{Laboratory breeding of Ancistrura nigrovittata}

Egg storage. The eggs for the laboratory breeding were obtained from wild type animals (caught in the surrounding area of Thessaloniki, Greece) or the F1generation (reared in the laboratory, Göttingen) of Ancistrura nigrovittata (Brunner von Wattenwyl, 1878). Fine sand or Vermiculite (Deutsche Vermiculite Dämmstoff $\mathrm{GmbH}$ ) in small containers on the floor of the rearing cages was used as substrate for egg deposition. The eggs were stored in petri dishes with Vermiculite until hatching. To prevent the eggs from drying out it is of particular importance to keep the soil under constant moisture. For embryo development the eggs were kept at $25^{\circ} \mathrm{C}$ for one to four month until entering the diapa use. When the eye spot was clearly seen through the egg sheath, using bright light from below and a binocular stereoscope for optical control, the eggs (ca. 30 eggs per petri dish) were stored at approximately $5^{\circ} \mathrm{C}$ for six month. Subsequently, the eggs were kept at ${ }^{\circ} \mathrm{C}$, where the hatching started (in the petri dish) not later than 2 weeks.

Rearing of juvenile stages. The hatchlings were transferred from the petri dish to a small cage $(15 \times 12 \times 12 \mathrm{~cm}$ covered with plastic mesh) in groups of 12 individuals at maximum. Small cages were used to increase the chance for the animals of finding food, which consisted of leafs of Rubus idaeus, Prunus spinosa, Cornus sanguinea, Rosa canina, Carpinus betulus and Rubus fruticosus. The latter was mostly fed during the winter time. A full nutrient concentrate for crickets (Nekton) served as additional food source. The temperature was maintained at 21 to $23^{\circ} \mathrm{C}$. Further heat and light apart from natural daylight was partially applied with a normal light bulb and a heating lamp (Osram siccatherm, 250 watt) at a distance of ca. $60 \mathrm{~cm}$ to the cage. By the time the animals reached the fourth or fifth juvenile stage ( 5 stages in total) a bigger cage $(53 \times 22 \times 30 \mathrm{~cm})$ was used for rearing groups of 25 to 30 individuals. Animals used in the experiment had to be adult for at least 2 weeks and the front legs (bearing the ears) were inspected to be undamaged. 


\subsection{Animal preparation}

The experimental animal was briefly anaesthetised with $\mathrm{CO}_{2}$ (ca. 2 min.) before fixing its ventral side to a plastic holder using a wax-colophonium mixture (2:1). The head capsule was bent with its mandibles touching the thorax and fixed to the holder by its antennae and genae. This provides a nearly horizontal brain position. Hind and middle legs were fixed to the plastic holder, while the tarsi of the front legs were attached to wires in a normal standing position.

In order to expose the brain the vertex and epicranium of the head capsule had to be removed first. The muscles were extracted by cutting the lateral sclerites, where the muscles are attached to. To ensure stable recording conditions in the head capsule, the gastrointestinal tract was constricted with a twine and then stretched until the tension minimised all disturbing movements. The brain was stabilised with a pretzel-like bended steel needle (minutien pin) from below. A small crump of collagenase (Sigma) was liquefied on a piece of cellulose and laid on the air exposed brain tissue for approximately 18 seconds. To remove remains of collagenase from the brain several washes with a saline solution (see Appendix and Fielden 1960) followed. This method simplifies the penetration of the brain sheath. From now on the brain was covered with saline during the whole course of the experiment. To reduce the disturbance by potential trachea or gastrointestinal tract movement a small metal ring (bended minutien pin held by a micromanipulator) was gently placed on top of the brain.

The experiments comply with the "Principles of animal care", publication No. 8623, revised, 1985 of the National Institute of Health, and also with the current laws of Germany.

\subsection{Stimulation}

\subsubsection{Experimental setup}

The prepared animal was placed with the plastic holder on a defined spot in an anechoic chamber. To exclude disturbing electric fields during experiments the chamber was also designed as a Faraday cage. Two dynamic loudspeakers (Dynaudio DF 21, 2-50 kHz) were set on both sides of the preparation at a distance of $37 \mathrm{~cm}$. The acoustic stimuli consisted of sinusoidal tones and white noise 
generated with a computer-controlled stimulator (Lang et al. 1993). This program also controlled carrier frequency, intensity and pattern of the stimuli and routed these via an amplifier to the speakers. The brain was magnified with a binocular (Wild M5A) and illuminated with an 85 watt cold-light source (Philips, Type 13194).

\subsubsection{Acoustic stimuli}

Search-program. In order to identify neurons that process auditory stimuli white noise was continuously presented at $70 \mathrm{~dB}$ SPL (pulse dur.: $50 \mathrm{~ms}$; pause dur.: $400 \mathrm{~ms}$ ) and $90 \mathrm{~dB}$ SPL (pulse dur.: $20 \mathrm{~ms}$; pause dur.: $400 \mathrm{~ms}$ ). In the late experiments, an artificial duet (male song at $70 \mathrm{~dB}$ SPL plus female answer at $60 \mathrm{~dB}$ SPL - see below for stimulus paradigm) was added to the search program.

Stimuli with varying intensity and carrier frequency. The categorisation of neurons due to their physiological properties is mainly based on a program that consisted of stimuli at 4 different frequencies $(8,16,28$ and $38 \mathrm{kHz})$. The pulses (dur.: $50 \mathrm{~ms}$; rise/fall time: $2 \mathrm{~ms}$; pause: $250 \mathrm{~ms}$ ) of each frequency increased in amplitude from 30 to $90 \mathrm{~dB}$ SPL in steps of $10 \mathrm{~dB}$ and were repeated 5 times at all intensities. In the late experiments, pulses presented at $00 \mathrm{~dB}$ SPL were added prior to each intensity series to the program to acquire information about the level of the neuron's spontaneous activity immediately before each frequency tested. In the following, this acoustical program will be referred to as "standard stimuli".

In another acoustic program an artificial male calling song was presented at 8 , 16,28 and $38 \mathrm{kHz}$. It consisted of a series of 8 pulses (pulse dur.: $7 \mathrm{~ms}$; rise/fall time: $0.5 \mathrm{~ms}$; pause dur.: $22 \mathrm{~ms}$ ) that were followed by a single trigger pulse (pulse dur.: $7 \mathrm{~ms}$; rise/fall time: $0.5 \mathrm{~ms}$ ) after a constant pause of $328 \mathrm{~ms}$ (also see Figure 1 for the natural male calling song). The intensity was increased in steps of $10 \mathrm{~dB}$ between 30 and $90 \mathrm{~dB}$ SPL (each intensity step was repeated three times; pause between cycles was ca. $278 \mathrm{~ms}$ ).

Temporal-programs. In order to test a neuron's responses to variations of temporal patterns, stimuli were presented that were either varied in pulse duration or pause duration while keeping the unchanged parameter at natural values. For this purpose white noise (70 dB SPL) was used, which was also the case in the behavioural experiments performed by Dobler et al. (1994a). The overall pulse group was kept at a constant value of ca. $210 \mathrm{~ms}$ (natural duration of the male song pulse 
series that has been tested to be behaviourally most effective - see Dobler et al. 1994a; each cycle was repeated five times and separated by pauses of $300 \mathrm{~ms}$ duration). Consequently, with varying pulse/pause durations also the number of pulses presented varied. The pulse duration varied between 2 and $25 \mathrm{~ms}$ and the presentation was as follows (constant pause dur. of $22 \mathrm{~ms}$; rise/fall time of the pulses: $0.5 \mathrm{~ms}$; randomised order; pulse dur./number of pulses): 02/9, 04/9, 07/8, $10 / 7,15 / 6$ and $25 / 5$. The stimulus paradigm with variations in pause duration was composed as follows (constant pulse duration of $7 \mathrm{~ms}$; rise/fall time: $0.5 \mathrm{~ms}$; randomised order; pause dur./number of pulses): 02/23, 05/18, 10/13, 15/10, 22/8, $35 / 6$ and $61 / 4$. To standardise the effect of adaptation, a $70 \mathrm{~dB}$ SPL white noise pulse $(500 \mathrm{~ms}$ duration) was given prior $(550 \mathrm{~ms})$ to the first stimulus cycle. Furthermore, the first pulse/pause pattern (not evaluated in the later processing) was repeated at the end of the paradigm.

Stimuli with variations in an artificial duet. Another program aimed to measure the carrier frequency dependence of the auditory neuron's response to the female reply in a conspecific duet. For this purpose an artificial duet was presented. The duet consisted of the male calling song (same stimulus pattern as described above; $70 \mathrm{~dB} \mathrm{SPL}$ ) plus the female answer pulse ( $3 \mathrm{~ms}$ pulse duration; rise/fall time: $0.5 \mathrm{~ms}$; $60 \mathrm{~dB}$ SPL) after a constant pause of $35 \mathrm{~ms}$ (corresponding to a distance between the duetting partners of approximately $1.5 \mathrm{~m}$ - see chapter 2.5 for the distance measurements). The carrier frequency was only varied for the female reply pulse (12, $16,20,24,28,34,38$ and $42 \mathrm{kHz}$ ) and each duet cycle was repeated 5 times at each carrier frequency (pause between cycles: $300 \mathrm{~ms}$ ). To standardise the effect of adaptation also the white noise pulse in the beginning as well as the repetition of the first stimulus cycles at the end of the paradigm was presented (see above).

In another program an artificial duet (male song at $16 \mathrm{kHz}$ and female reply at $28 \mathrm{kHz}$ carrier frequency) was presented and the pause between the female reply and the male trigger pulse was varied $(5,15,25,35,45,65$ and $85 \mathrm{~ms}$ pause duration). Each duet cycle was repeated 5 times for every pause duration tested (pause between cycles: $300 \mathrm{~ms}$ ). To standardise the effect of adaptation also the white noise pulse in the beginning as well as the repetition of the first stimulus cycles at the end of the paradigm was presented (see above). 
Stimuli to test a neuron's response to vibrations. A rough test to determine a neuron's response to vibrational stimuli was done by slightly scraping against the edge of a metal plate at the experimental setup. This stimulus did not evoke any response in a purely "auditory" neuron.

\subsubsection{Calibration}

The sound pressure (below also referred to as intensity) of each carrier frequency was regularly calibrated (dB SPL (RMS) re $2^{*} 10^{-5} \mathrm{~Pa}$ ) with a Brüel \& Kjaer amplifier (2610) and a condenser microphone (Brüel \& Kjaer: 1/2 inch 4133 or $1 / 4$ inch 4135). The microphone was placed at the position of the preparation and a constant sinusoidal tone was repeatedly measured, giving an accuracy of $+/-2 \mathrm{~dB}$.

\subsection{Recording and staining techniques}

\subsubsection{Intracellular recording}

Thick-walled borosilicate capillaries with inner filaments (OD/ID $=1.0 / 0.58 \mathrm{~mm}$ and $76 \mathrm{~mm}$ length, 1B100F-3, World Precision Instruments) were used for intracellular or quasi-intracellular recordings (Mcllwain \& Creutzfeld 1967). The tips were produced with a Flaming/Brown micropipette puller (Model P-97, Sutter) and had resistances of 80-150 $\mathrm{M} \Omega$ when filled with a 3 molar potassium acetate solution. Two different microelectrode holders were used. A silver wire holder (custom made) was used during most of the experiments. In a few experiments a half-cell pellet holder (silver/silver chloride, MEH3S10, World Precision Instruments) was used for the metal/liquid coupling. The holder was attached to a micromanipulator in the anechoic chamber that allowed free movement along 3 axes. A grounded $\mathrm{Ag} / \mathrm{AgCl}$ wire was immersed into the hemolymph of the abdomen and was used as the reference electrode. The recordings were amplified tenfold by a DC amplifier (custom made) and stored on a digital recorder (PC204A, SONY) together with the envelope of the stimulus.

The search for auditory neurons concentrated on the auditory neuropil in the lateral protocerebrum of the brain. All recordings were performed at room temperature and monitored during the experiment with a $20 \mathrm{MHz}$ oscilloscope (Gould DSO 1604). 


\subsubsection{Neurobiotin staining}

In order to stain a nerve cell during an intracellular recording, the tip of the capillary was backfilled with $5 \%$ neurobiotin (Vector laboratories) in 1 molar potassium acetate (Kita \& Armstrong 1991). The shaft was filled with a 3 molar potassium acetate solution. The neurobiotin was then applied to the neuron with a constant positive current of ca. $0.5-1.0 \mathrm{nA}$. Subsequent to the experiment the nervous system was dissected, fixed in 4\% paraformaldehyde (dissolved in PBS) and the neurobiotin tracer was coupled to Cy3-conjugated streptavidin (Jackson Immuno Research laboratories) or fluorescein streptavidin (FITC, Vector laboratories) according to a modified scheme by Mesce et al. 1993 (see Appendix for the exact procedure). Then, the brain was dehydrated with successive changes in ethanol/aqua dest., cleared with methylsalicylate (Merck) and analysed with a conventional fluorescence microscope (Leica, DM-RB) or a confocal scanning microscope (Leica, DM-RE TCS SP2). Drawings of the cells were either made using a LEICA microscope (Dialux 20) and a drawing tube, or a fluorescence microscope (Zeiss Axioskop) equipped with a Spot CCD camera (Intas).

\subsection{Data evaluation}

In this study more than 350 auditory neurons were intracellularly recorded and over 100 of them were clearly classified according to their morphological and/or physiological properties. The stored physiological data of all neurons was digitised with TurboLAB (Stemmer TurboLAB 4.0) via an analog-digital converter (DT 2821) at $10 \mathrm{kHz}$. The evaluation of the exact spike timing and the averaging of original recordings (to reduce the signal-to-noise ratio) were achieved using the NEUROLAB program (Hedwig \& Knepper 1992). Spikes were counted with programs (custom made) implemented in TurboPascal in a window of e.g. 15 to $150 \mathrm{~ms}$ (intervals between spikes being not longer than $50 \mathrm{~ms}$ ) after the onset of the stimulus for $50 \mathrm{~ms}$ pulses. This window was enlarged to $400 \mathrm{~ms}$ for the models of the male song structure or to $700 \mathrm{~ms}$ for the male song at varying carrier frequencies.

Data are generally given as mean plus standard error (mean $\pm \mathrm{SE}$ ), if they are averages of the means of several individuals (at least 3 individuals). Otherwise, data will be given as mean and standard deviation (mean $\pm S D$ ). The number of specimen is always given as "N", whereas single values are indicated as " $n$ ". 
The box plots show the medians (black bar; values indicated in the figure), the upper and lower quartiles (H-spread) and the whiskers (1.5 times the $\mathrm{H}$-spread or the minima and maxima of the respective values). Statistics were performed using a freeware program (http://faculty.vassar.edu/lowry/VassarStats.html). The threshold value for a tuning curve was defined as the intensity of sound of the respective frequency that elicited one spike above the spontaneous activity level in three out of five stimuli (also see Stumpner 1997). The values were calculated by interpolating from responses to succeeding intensity steps. If in intensity scans spiking occurred at least to 4 out of 5 stimuli with short latency and low variance (standard deviation lower than $25 \%$ of the mean) without the threshold criterion (number of spikes) being reached at this or the next higher intensity step, a threshold value was nevertheless extrapolated from this and the preceding intensity step if possible. This occurred only in AN1 and especially at ultrasonic frequencies, since responses remained weak due to combined excitatory and inhibitory influence. If no identifiable response was elicited up to the highest intensity tested (90 dB SPL), the threshold value was set to $100 \mathrm{~dB}$ SPL.

The distance reference for the duetting partners (light blue bar in Figure 28B) expressed by the latency to the male trigger in correlation to the spikes of TN1 was calculated by adding: (i) the medium response latency of females (25 ms, minus $0.57 \mathrm{~ms}$ for the spacing of the speaker with which females were stimulated - Dobler et al. 1994a), and (ii) a sound delay caused by the distance - (distance/velocity of sound value has to be doubled, since the sound of the trigger pulse propagates to the female and the reply back to the male).

The data was post-processed using EXCEL (Microsoft), CorelDRAW X4, Corel PHOTO PAINT X4 and Adobe Photoshop (7.0.1).

\subsection{Immunohistochemistry}

Dissected brains were fixed with $4 \%$ paraformaldehyde dissolved in phosphate buffer for 2 hours at room temperature, embedded in albumin-gelatine and postfixed in $4 \%$ paraformaldehyde overnight at $4^{\circ} \mathrm{C}$. Frontal se ctions $(=$ horizontal in respect to the neuro axis) of $40 \mu \mathrm{m}$ thickness were cut with a vibrating blade microtome (VT 1000 S, Leica). The tissue sections were permeabilised in PBS (see Appendix) containing $1 \%$ Triton $\mathrm{X}-100$ (Sigma) for 2 days at $8^{\circ} \mathrm{C}$. Nonspecific binding of the antibody was blocked in $2 \%$ normal goat serum (GE-Healthcare) and $3 \%$ bovine 
serum albumin (MPI Biomedical) dissolved in PBS-1\% Triton for 2 hours at room temperature. For neuronal staining, anti- HRP serum (rabbit a-HRP; Sigma) was applied to the sections at a dilution of $1: 500$ at $8^{\circ} \mathrm{C}$ for 2 days. After washing several times with PBS, a Cy2-coupled secondary antibody (Cy2 goat a-rabbit IgG, Rockland) at a dilution of 1:300 was used to visualise immunoreactivity. For nuclear staining, tissue sections were incubated for 30 minutes at room temperature with $100 \mu \mathrm{g} / \mathrm{mL}$ 4'-6-diamino-2-phenylindole (DAPI; Sigma) dissolved in PBS. Following several washes in PBS and transfer to PBS with glycerol (1:1), fluorescence was analysed with a conventional fluorescence microscope (Zeiss Axioskop) equipped with a Spot CCD camera (Intas). 


\section{Results}

All intracellular recordings of auditory neurons were carried out in the lateral protocerebrum of the brain, where the axons of ascending neurons have their major arborisations. For the anatomical presentation of the lateral protocerebrum and other prominent neuropils horizontal sections of a brain of Ancistrura nigrovittata were prepared and subsequently labelled with anti-HRP (anti-horseradish peroxidase) serum and the nuclear marker DAPI (4'-6-diamino-2-phenylindole) (Figure 4). AntiHRP is a specific neuronal surface marker (Jan \& Jan 1982) that stains cell bodies and neurites of neurons (green fluorescence). DAPI, on the other hand, binds to the DNA of cell bodies (blue fluorescence; Wilson et al. 1990). Therefore, the combination of both markers can be used to distinguish between somata of neurons in the cortex and their neurites that form the distinct neuropil regions. For comparison also a schematic overview of all important brain compartments is shown (strongly modified from Müller et al. 1997). The auditory neuropil in the lateral protocerebrum is indicated by a white/black dashed circle. All morphological descriptions in the brain will be given as it compares to the neuronal axis of the central nervous system.

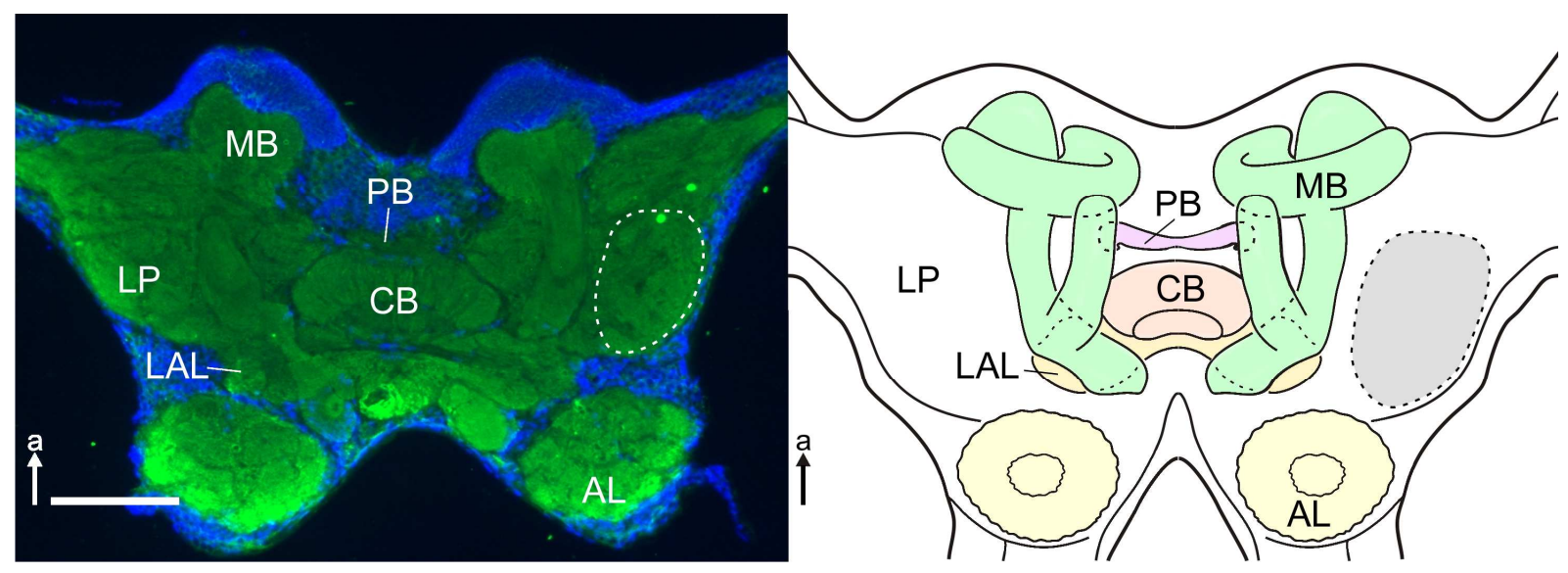

Figure 4: Left: Horizontal section through the brain of Ancistrura nigrovittata labelled with anti-HRP serum (green) and DAPI nuclear stain (blue) showing most of the important neuropils. Bar represents $250 \mu \mathrm{m}$. Right: Schematic drawing of the neuropils seen in the fluorescent staining (strongly modified after Müller et al. 1997). White dashed line (left) and grey area (right) indicate the auditory neuropil. $A L=$ antennal lobe, $C B=$ central body, $L A L=$ lateral accessory lobe, $L P=$ lateral protocerebrum, $\mathrm{MB}=$ mushroom body, $\mathrm{PB}=$ protocerebral bridge, $\mathrm{a}=$ anterior .

In the first part of this chapter axonal recordings of ascending neurons will be compared to recordings collected near the spike generating zone in the thorax (thoracic recordings were mainly performed by Andreas Stumpner) and differences as well as similarities concerning the physiology will be shown. In addition, 
recordings of a yet unidentified ascending neuron will be presented. In the second part of this work the morphology and the physiology of various auditory interneurons will be demonstrated for the first time in the brain of a bush cricket. The response properties of individual brain neurons will then be compared with behavioural data collected in the past (mainly by Susanne Dobler).

\subsection{Ascending neurons}

\subsubsection{Ascending neuron 1 (AN1)}

AN1, with its comparatively thick axon and dense axonic arborisations, can be easily recorded in the lateral protocerebrum of the brain. Distinct physiological properties allow a clear classification already during the experiments. These properties consist of action potentials with axonic appearance and a strong phasictonic response to low amplitude $16 \mathrm{kHz}$ stimuli.

Confirmation of morphology. Apart from the analysis of a possible change of a neuron's physiology, when recorded in or close to the dendritic input region, also a confirmation of morphology was performed for some ascending neurons. In Figure 5 a fluorescent (Cy3) labelled axon of AN1 in the brain is displayed (Figure 5 left) and compared to a drawing of axonal arborisations obtained from a staining performed in the thorax by A. Stumpner (Figure 5 right). Typical features of this interneuron can be recognised. These are: (i) the restriction of all axonal arborisations to the lateral protocerebrum (unlike all other ascending interneurons except AN4) (ii) the two major collaterals - a caudal and an anterior branch (marked by arrowheads in Figure 5 left) (iii) the characteristic "circle" formed by the finer collaterals of the anterior branch (also compare to Stumpner 1997 and Figure 3 of this study). The comparative analysis of these AN1-stainings obtained in different individuals (and different penetration sites - thorax vs. brain) reveals uniform branching patterns in congruent regions of the protocerebrum. 

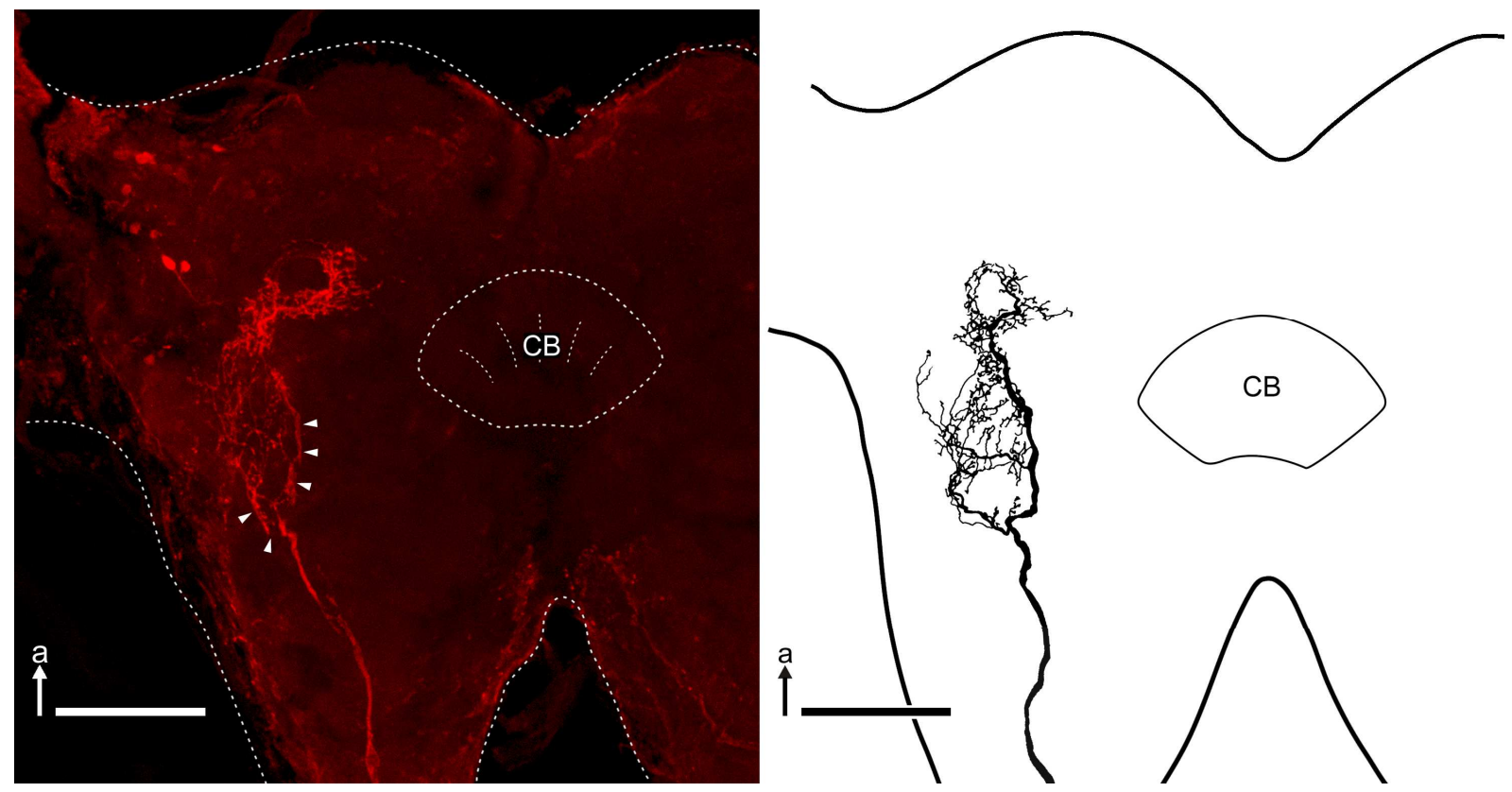

Figure 5: Morphology of two AN1s in the brain of $A$. nigrovittata in relation to the central body (CB). Bar represents $200 \mu \mathrm{m}$. Left: Cy3-staining of AN1 recorded in the brain. Arrowheads mark the caudal and anterior collaterals (due to the subsequent developing procedure of the Cy3-staining parts of the collaterals are damaged). Right: Drawing of AN1's brain arborisations from a staining done in the thorax by $\mathrm{A}$. Stumpner. $\mathrm{a}=$ anterior.

Intensity dependence of AN1's response at various carrier frequencies. Recordings of AN1 in the brain show spikes without any graded potentials indicating a penetration far away from the spike generating zone (Figure 6A). When a complete duet (including the female reply) of $A$. nigrovittata is presented, spiking activity can only be observed to the male calling song. Stimulation of AN1 with 50 ms pulses for 8, 16, 28 and $38 \mathrm{kHz}$ at increasing intensities (see chapter 2.3 .2 for stimulus parameters; in the following referred to as "standard stimuli") reveal strongest responses for 8 (70 - $80 \mathrm{~dB}$ SPL) and $16 \mathrm{kHz}$ (50 dB SPL) (Figure 6B). In contrast, ultrasonic frequencies evoke the lowest response. When comparing the mean spike counts recorded in the thorax (Figure 6B left) and in the brain (Figure 6B right) a close match of spike number for the respective frequency and intensity is found. One major difference becomes evident, though, at the highest intensities tested (80 and $90 \mathrm{~dB}$ SPL) - the responses of AN1 in the brain are relatively lower at all frequencies. This is especially obvious at ultrasonic frequencies. When looking at single AN1 responses at $16 \mathrm{kHz}$, one can see great variability at high intensities in thoracic recordings (Figure $6 \mathrm{C}$ ). This variability in spiking activity results in a higher mean activity that differs from the mean response of AN1 recorded in the brain. 
A
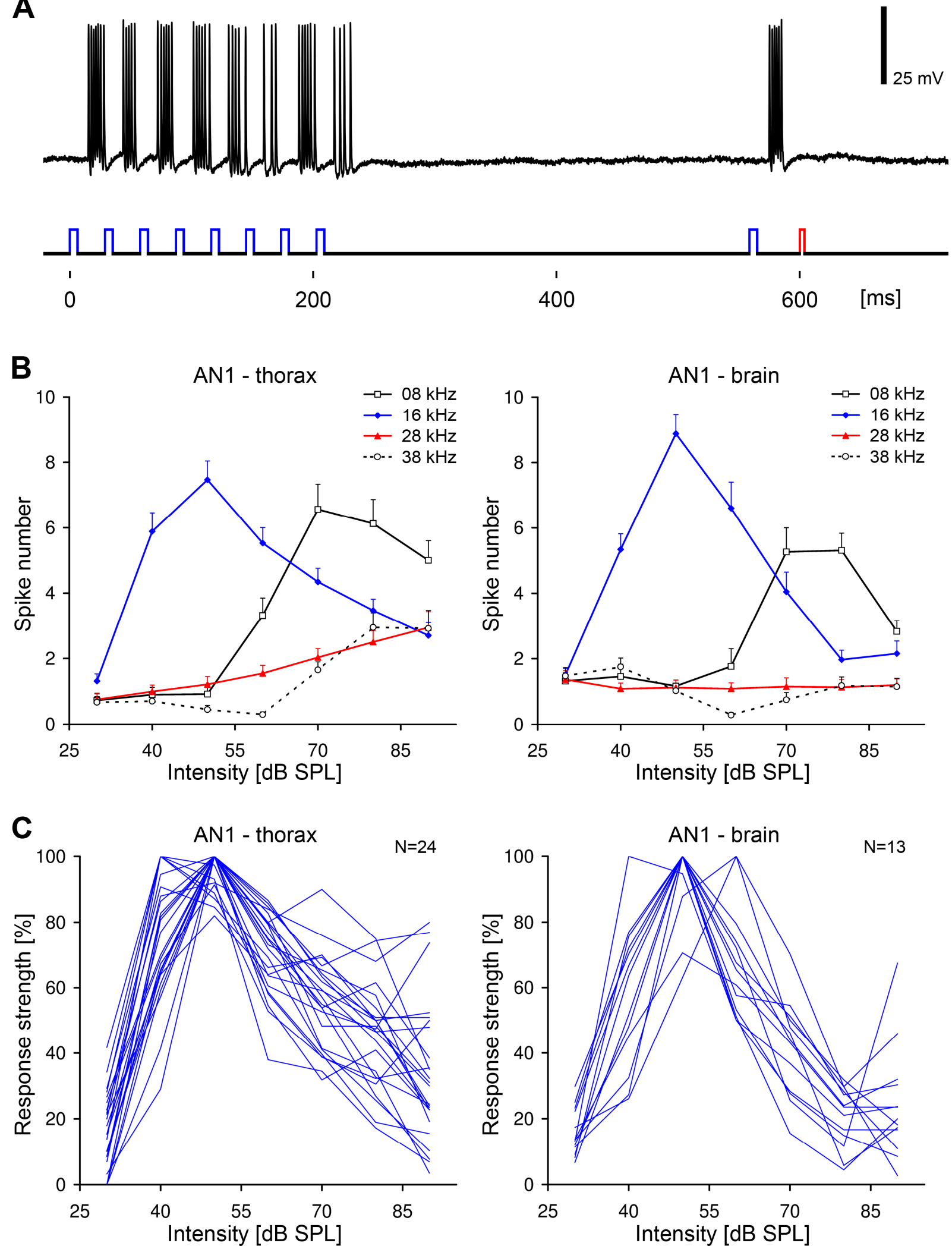

Figure 6: Response of AN1 to acoustic stimuli. Blue colouration indicates the carrier frequency of the male song $(16 \mathrm{kHz})$ and red the carrier of the female reply $(28 \mathrm{kHz})$ (prothoracic data modified from Stumpner \& Molina 2006). A: Example recording of a response to an artificial duet of $A$. nigrovittata (male song at $70 \mathrm{~dB}$ SPL; female answer at $60 \mathrm{~dB}$ SPL; see chapter 2.3.2 for stimulus paradigm). B: Intensity dependent response of AN1 recorded in the prothorax (left: $\mathrm{N}=21-24$; means and SE) and in the brain (right: $\mathrm{N}=13$; means and $\mathrm{SE}$ ) at $8,16,28$ and $38 \mathrm{kHz}$ (pulse dur.: $50 \mathrm{~ms}$; "standard stimuli"). C: Normalised response for different individuals recorded at $16 \mathrm{kHz}$ only (pulse dur.: $50 \mathrm{~ms}$ ). 
A comparison of the female behaviour and the relative spike numbers of AN1 to $16 \mathrm{kHz}$ at increasing intensity is shown in Figure 7. The spike numbers closely correlate with the behaviour although minor differences are found. For 30 and $40 \mathrm{~dB}$ SPL AN1's activity is higher than the behavioural data. Since female behaviour has not been tested at 70 - $90 \mathrm{~dB}$ SPL (see red dashed line in Figure 7), a comparison to AN1's response at these intensities can not be drawn.

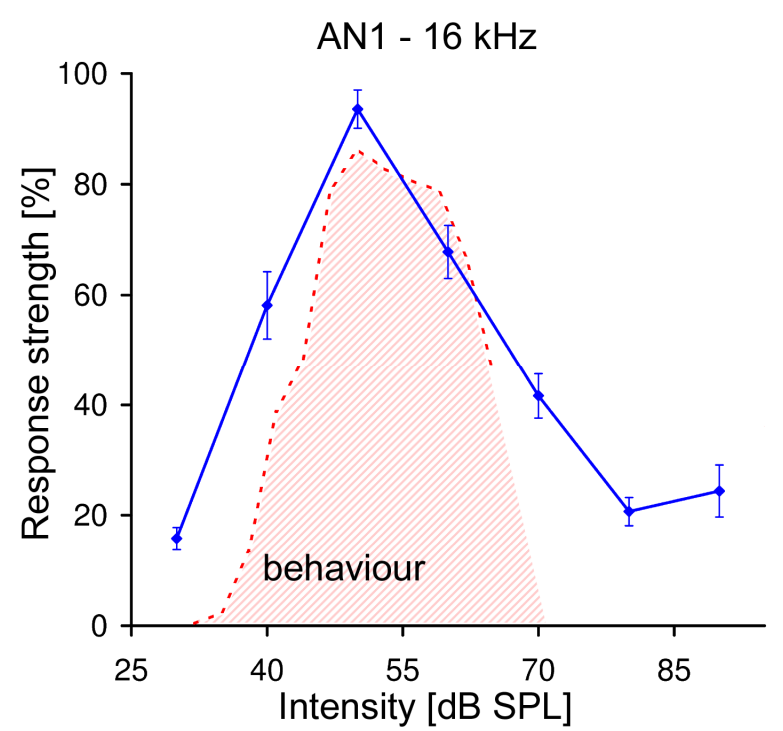

Figure 7: Intensity response function of AN1 (relative spike numbers; brain recording; $\mathrm{N}=13$; means and SE) to $16 \mathrm{kHz}$ stimuli. Red shaded area indicates the female behaviour $(\mathrm{N}=5)$ to the respective carrier frequency (modified from Dobler et al. 1994b).

Responses of AN1 to varying temporal parameters. Simplified male model songs were presented to identify possible filter mechanism of AN1 for relevant song parameters. In Figure 8A thoracic and brain recordings are compared for varying pause durations. At all parameters tested thoracic recordings show higher spike numbers, which are most likely due to depolarisation by the penetration. But the response strength is widely similar to brain recordings with the clearest difference at very short pause durations $(2 \mathrm{~ms})$. The strongest activity was recorded for $10 \mathrm{~ms}$ pauses with optimum type character. The specificity of the female's response, however, cannot be explained by the spiking activity of AN1. A comparison of AN1's responses to different pulse durations is displayed in Figure 8B. Besides an approximately $20 \%$ higher spike number in the thoracic recording than in brain recordings, a large difference is found at very short pulse durations (2 ms). AN1 shows a comparably low response to short pulses, like e.g. the female answer pulse (marked as a red data point in Figure 8B right). Hence, AN1 exhibits filter characteristics that exclude very short pulses (when recorded in the brain) and its spiking strength correlates closely with the female behaviour up to pulse durations of 
$10 \mathrm{~ms}$. This filtering characteristic in AN1 completely vanishes when penetrated close to the spike initiating zone.
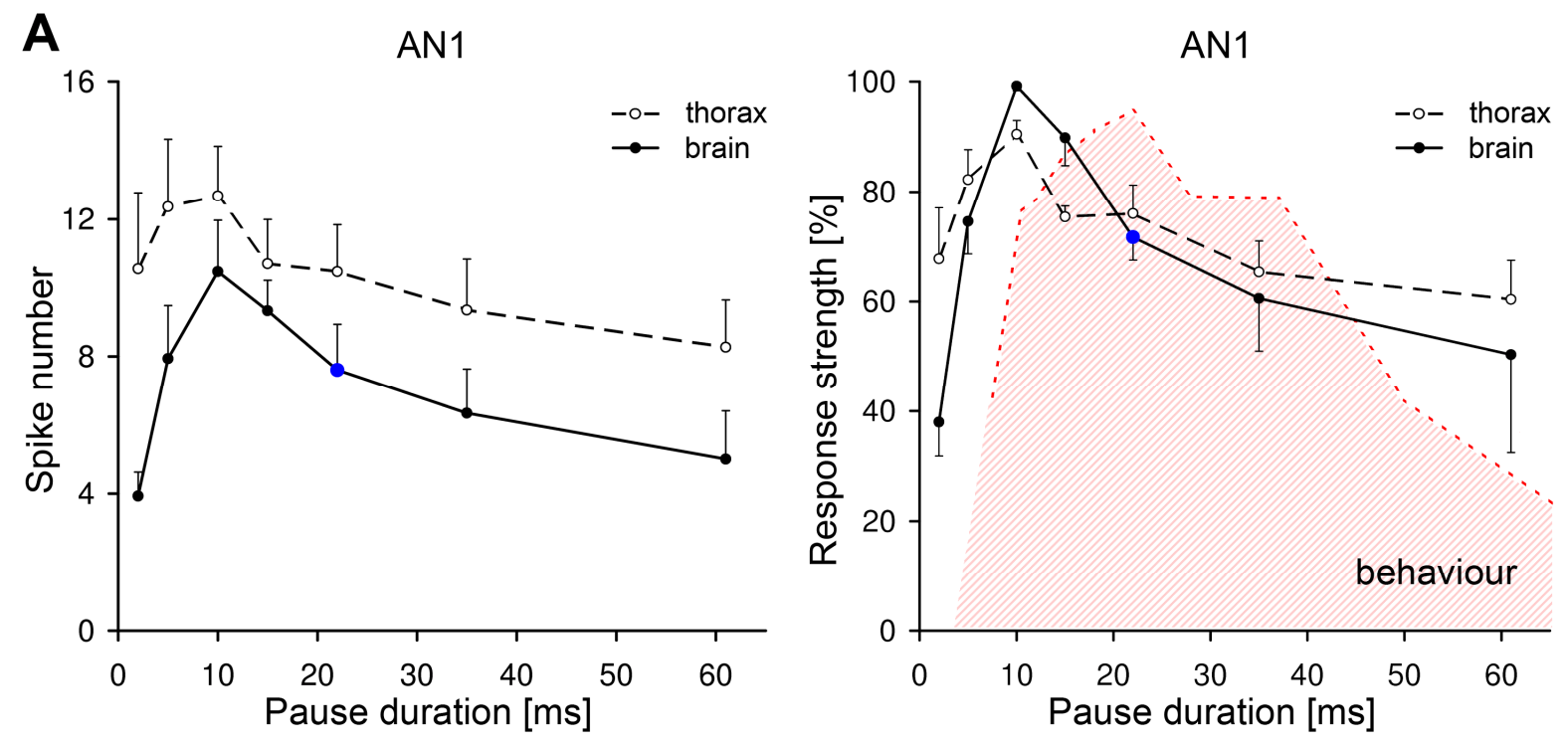

B

AN1
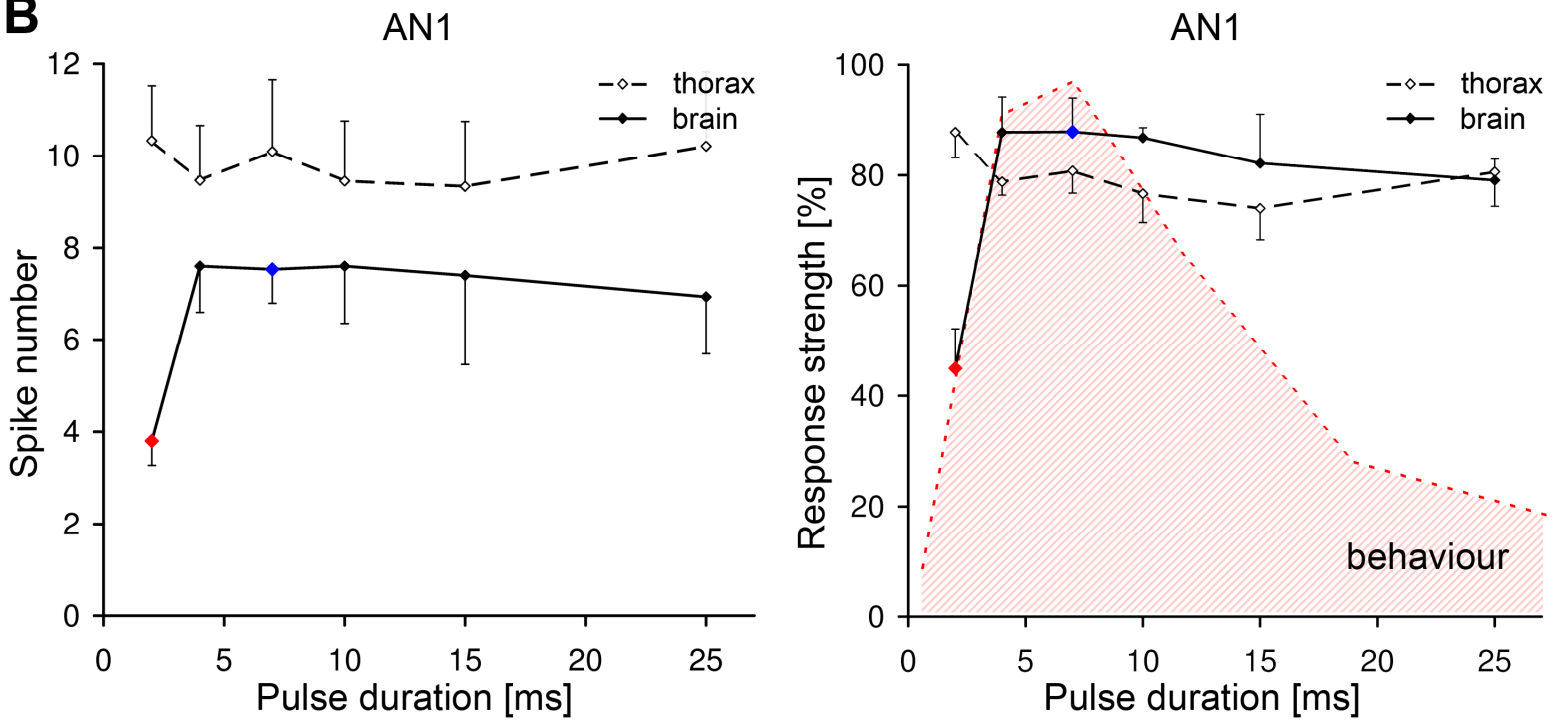

Figure 8: Mean response of AN1 compared in thoracic (dashed lines; $\mathrm{N}=12$; data modified from Stumpner \& Molina 2006) and brain (solid lines; $\mathrm{N}=3$ ) recordings to different temporal parameters (white noise; $70 \mathrm{~dB}$ SPL; see chapter 2.3.2 for stimulus paradigm; means and SE). Important natural parameters are emphasised by coloured data points (blue: male song pause/pulse duration; red: female's reply pulse duration). Red shaded area indicates the female behaviour ( $N=5$, modified from Dobler et al. 1994a). A: Spike number (left) and magnitude of response (right) to stimuli with varying pause duration. B: Spike number (left) and magnitude of response (right) to stimuli with varying pulse duration.

Difference in spike latencies. AN1 gets monosynaptical input from auditory afferents in the prothoracic ganglion (Stumpner 1997). Shortest spike latencies measured in the thorax lie at $15.2 \pm 0.4 \mathrm{~ms}$ (at $16 \mathrm{kHz}, 60 \mathrm{~dB} \mathrm{SPL}$; mean and SE) (Figure 9). Axonal recordings of AN1 performed in the brain have an approximate 
distance of $4.2 \mathrm{~mm}( \pm 0.3 \mathrm{~mm}$ - deviation depends on the searching spot in the area of the auditory neuropil in the brain and in the thorax) to the prothoracic recording site (measured from a photograph of a dissected CNS with the program ImageJ 1.410 plugin: NeuronJ; National Institutes of Health) and, therefore, spike latencies in the brain are markedly higher.

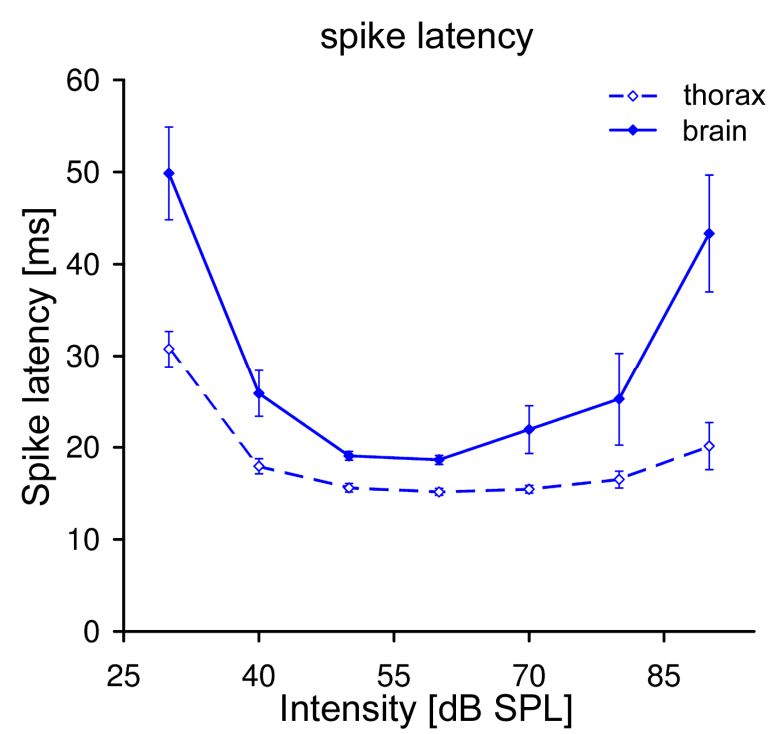

Figure 9: Mean spike latencies measured in thoracic and brain recordings at $16 \mathrm{kHz}(50 \mathrm{~ms}$ stimuli) for increasing intensities (thorax: $\mathrm{N}=23$ data modified from Stumpner 1997; brain: $\mathrm{N}=13$; means and SE).

Shortest spike latencies measured in the brain are $18.6 \pm 0.5 \mathrm{~ms}$ (Figure 9 at $16 \mathrm{kHz}$, $60 \mathrm{~dB}$ SPL; mean and SE). Thus, the travelling time of spikes within AN1 is about $3.4 \mathrm{~ms}$, which equals a conduction velocity of $1.2 \pm 0.1 \mathrm{~m} / \mathrm{s}$ (the deviation reflects the approximate error of the recording spot in each auditory neuropil - see above; errors dependent on the temperature and the size of the animals are disregarded since averaged latencies are compared). During stimuli outside the range of best response (e.g. at 30 and $90 \mathrm{~dB}$ SPL) the difference of spike latencies between both recording spots is considerably higher.

\subsubsection{Ascending neuron 2 (AN2)}

During a typical experiment the axon of AN2 is rarely recorded in the brain. In addition, AN2's physiology has no distinct characteristical traits that would ease a classification during an experiment when recorded in axonal portions. When no morphological data is available, only the combined evaluation of AN2's spiking activity to different acoustical test programs makes a classification possible. 
Intensity dependence of AN2's response at various frequencies. In Figure 10A two example recordings of AN2 are displayed. The response to an artificial duet can be very diverse between individuals. Only in one (out of four) individual the presentation of the male song evoked no action potentials in AN2, which was formally known from thoracic recordings for AN5-AG7 only (see chapter 3.1.4). Mostly few spikes are elicited. But a clear response to the female answer pulse is always found, which is contrary to the data obtained in the thoracic recording site (Stumpner \& Molina 2006).
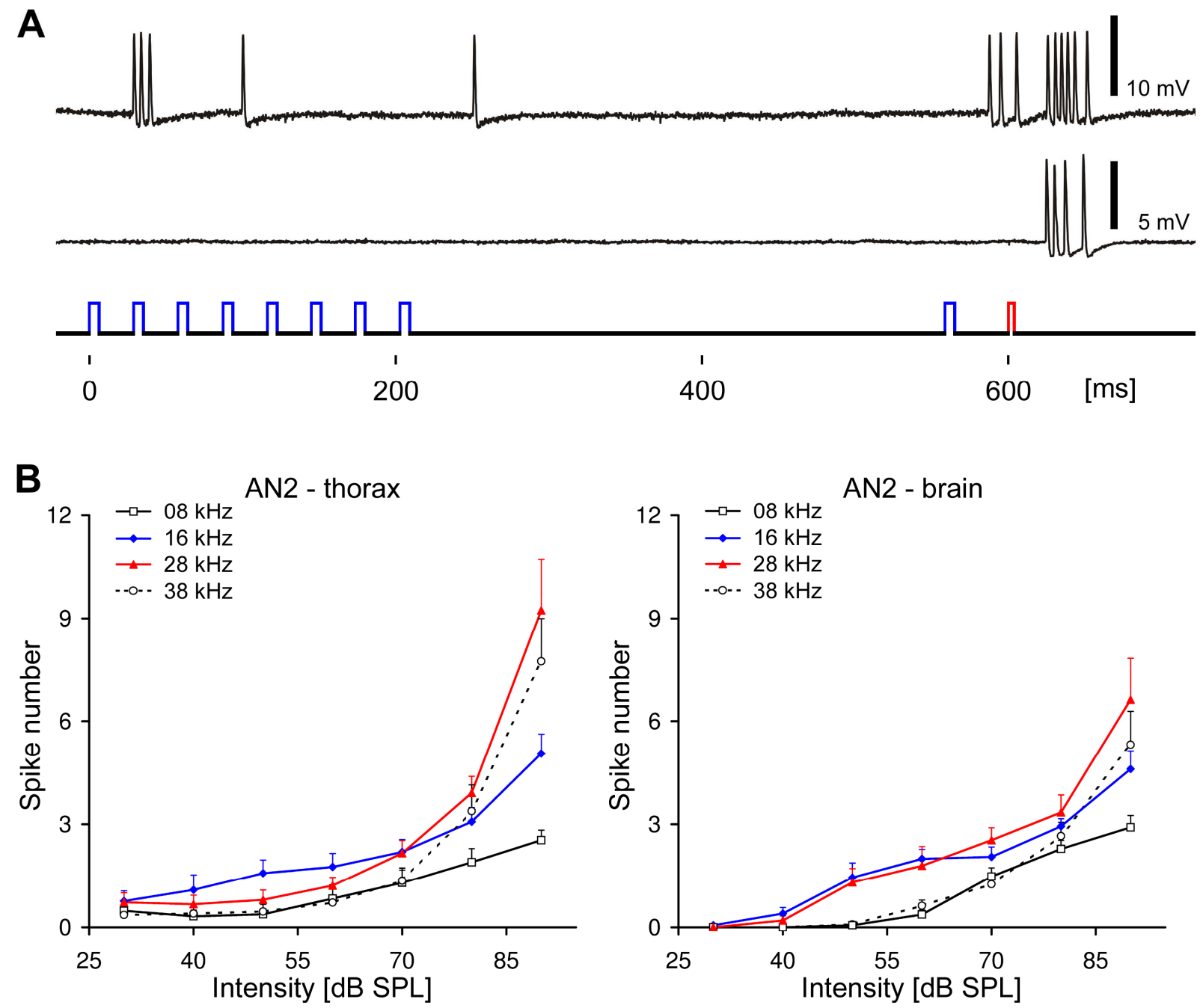

Figure 10: Response of AN2 to acoustic stimuli. Blue colouration indicates the carrier frequency of the male song $(16 \mathrm{kHz})$ and red colouration the carrier of the female reply $(28 \mathrm{kHz})$ (prothoracic data modified from Stumpner \& Molina 2006). A: Two different example recording of AN2's response to an artificial duet of $A$. nigrovittata (male song at $70 \mathrm{~dB}$ SPL; female answer at $60 \mathrm{~dB} \mathrm{SPL}$; see chapter 2.3.2 for stimulus paradigm). B: Response of AN2 to standard stimuli (see chapter 2.3.2). Left: Recordings from the prothorax ( $\mathrm{N}=10-11$; means and $\mathrm{SE})$. Right: Recordings from the brain $(\mathrm{N}=7$; means and SE). 
The intensity dependent response (Figure 10B) shows a strong activity at ultrasonic frequencies for high intensities which is more distinct in thoracic than in brain recordings. Contrary, at low intensities (at 50 to $60 \mathrm{~dB} \mathrm{SPL}$ ) responses to $16 \mathrm{kHz}$ always show a slightly higher spiking activity than the responses to other frequencies when recorded in the thorax. In the brain responses to $28 \mathrm{kHz}$ have analogue values but the intraindividual variability is very high. An overall comparison of thoracic and brain recordings reveal similar characteristics for all frequencies tested. Nevertheless, it is most likely that depolarisation due to penetration alters the spontaneous activity in AN2, since spiking also occurs to very low intensities at all frequencies tested (compare $30 \mathrm{~dB}$ SPL in Figure 10B left and right). In the brain recording site no spiking activity could be detected at this intensity.

Response to different frequencies of the female answer in an artificial duet. The presentation of a complete duet with varying carrier frequency of the female reply (see chapter 2.3.2 for stimulus parameters) reveals the strongest response to $28 \mathrm{kHz}$ and a steep roll off to lower and higher frequencies (Figure 11). Therefore, AN2 exhibits a strong filter for the female's carrier frequency at short pulses (and low intensities), which is contrary to the response to longer pulses as seen in the intensity response function above (Figure 10).

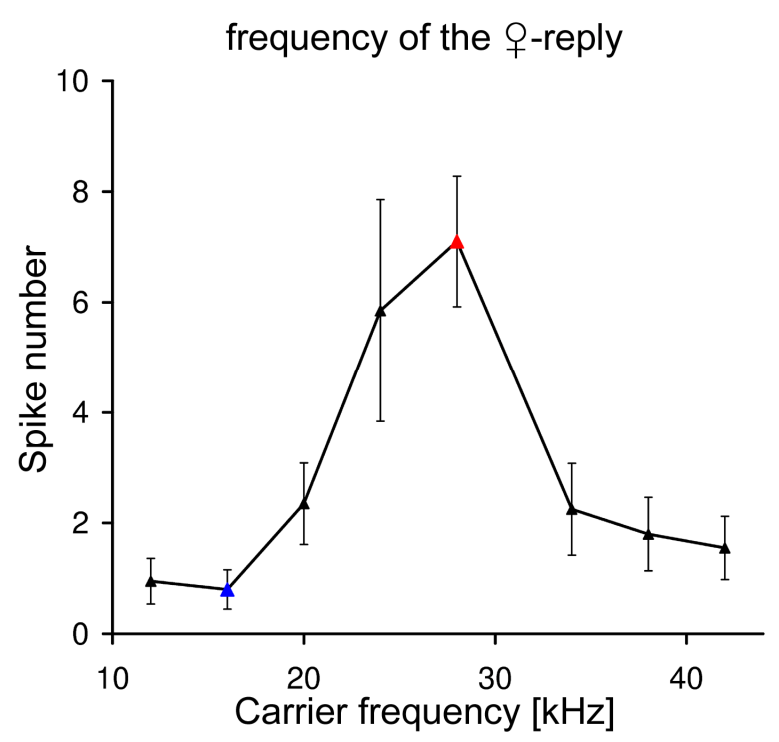

Figure 11: Mean response of AN2 (recorded in the brain) to the female answer with varying carrier frequency in an artificial duet $(\mathrm{N}=4$; see chapter 2.3.2 for stimulus paradigm; means and $\mathrm{SE})$. Blue data point indicates the male's natural carrier frequency and red data point the natural frequency of the female reply.

Response of AN2 to varying temporal parameters. The spiking activity in AN2 is increasing with increasing pause durations up to approximately 10 action potentials (at $60 \mathrm{~ms}$ pause duration - Figure 12A). This was already shown from thoracic 
recordings and could be confirmed in the brain although the mean spiking activity was stronger in the thorax at most pause durations tested.
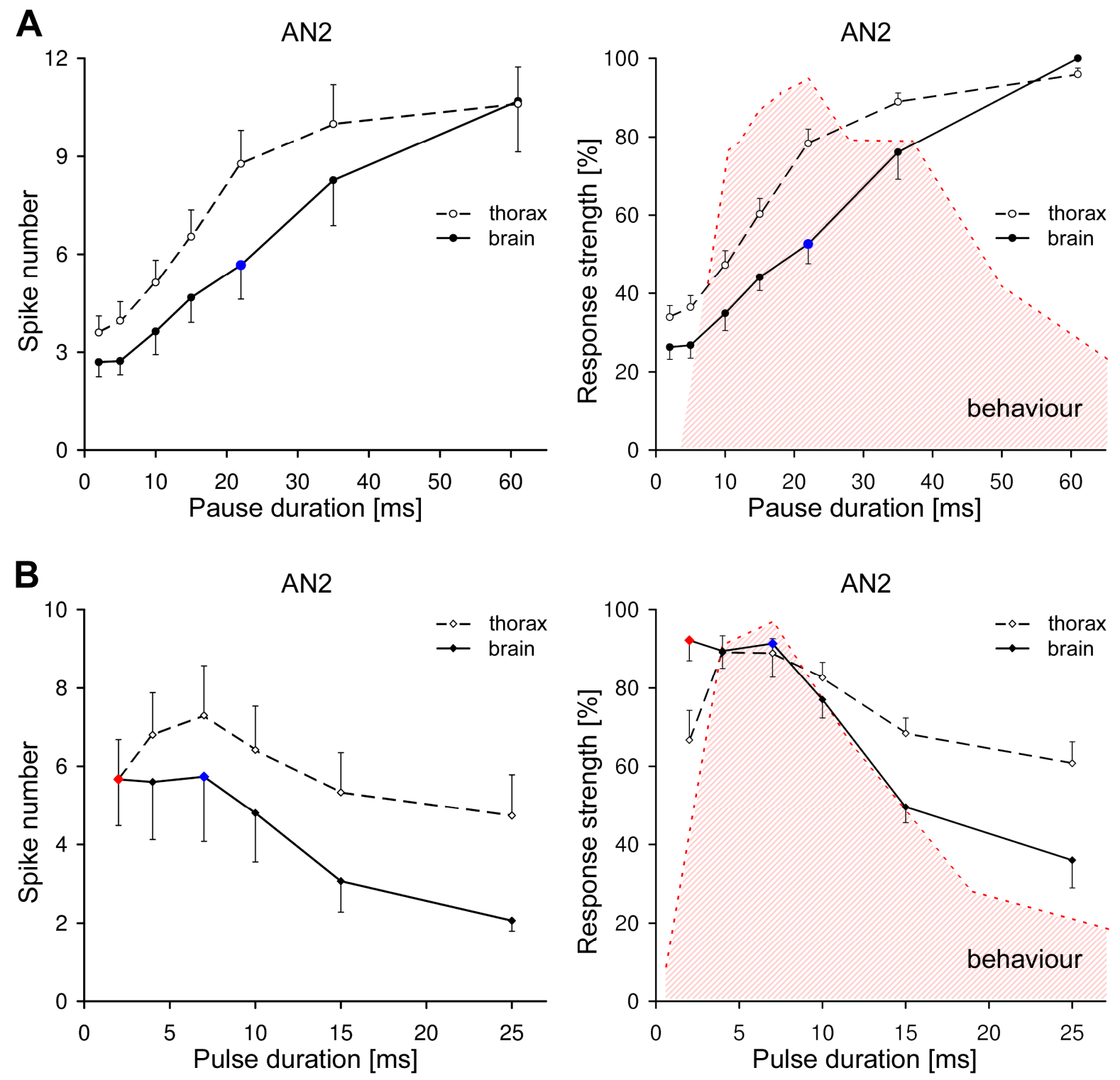

Figure 12: Mean response of AN2 compared in thoracic recordings (dashed lines; data modified from Stumpner \& Molina 2006) and brain recordings (solid lines) to different temporal parameters (white noise; $70 \mathrm{~dB}$ SPL; see chapter 2.3.2 for stimulus paradigms; means and SE). Important natural parameters are emphasised (blue: male song pause/pulse duration; red: female reply pulse duration). Red shaded area indicates female behaviour ( $N=5$, modified from Dobler et al. 1994a). A: Spike number (left) and magnitude of response (right) to stimuli with varying pause duration (thorax: $\mathrm{N}=16$; brain: $\mathrm{N}=6$ ). B: Spike number (left) and magnitude of response (right) to stimuli with varying pulse durations (thorax: $\mathrm{N}=12$; brain: $\mathrm{N}=3$ ).

From the data collected in the thoracic region AN2 was considered (among other ascending neurons) to play a role in the female preference for variations in pause duration (Stumpner \& Molina 2006). Data from the brain shows the best response of AN2 at the highest pause duration (steadily increasing response) where 
the behaviour is lowest (from the values tested; optimum type curve). However, the spiking activity of AN2 may still partly explain the decreased behavioural response to short pause durations (2 - $22 \mathrm{~ms})$, since the response in AN2 is also lowest. The decrease of the behavioural response to longer pause durations, though, cannot be explained by AN2's spiking activity. Concerning the pulse duration, AN2 exhibits a strong filter for short pulses that perfectly matches the behavioural response of females when compared to the data of brain recordings (Figure 12B). The response magnitude of AN2 obtained in thoracic recordings does not show such a close correlation with the female behaviour. This might be due to the considerably higher spike number that can be observed at most pulse durations tested. Whether this filter for short pulses contributes to the selective behavioural response of females to the natural male pulse duration remains questionable since best responses of AN2 are found to the female answer (only seen in brain recordings - see Figure 10A). The variability of spike number is very high among single individuals (even in brain recordings) whereas the variation in the response strength is low (note the standard error in Figure 12B left and right). The biggest difference between both recording sites is found at pulse durations of $2 \mathrm{~ms}$. Here, AN2s recorded in the brain have a more than $20 \%$ higher response strength compared to the thoracic recordings (whereas mean spike numbers are identically). High interindividual variability does not account to this difference in the mean of the response, since nearly all AN2s responded accordingly. This holds true for brain recordings as well as for thoracic recordings.

Difference in spike latencies. The shortest spike latencies were measured for $28 \mathrm{kHz}$ at $90 \mathrm{~dB} \mathrm{SPL}$. In thoracic recording the mean latency for these values is $17.6 \pm 0.6 \mathrm{~ms}(\mathrm{~N}=11$; mean and $\mathrm{SE})$. In brain recordings the latencies were $21.7 \pm 0.7 \mathrm{~ms}(\mathrm{~N}=7$; mean and $\mathrm{SE})$ and therefore, AN2 exhibits a conduction velocity of ca. $1.0 \pm 0.1 \mathrm{~m} / \mathrm{s}$.

Indications for the nature of a possible secondary auditory or vibratory input in AN2 via the extensive dendritic arborisations in the deutocerebrum (see Stumpner \& Molina 2006 and Figure 3) could not be seen by recordings in the brain. 


\subsubsection{Ascending neuron 3 (AN3)}

The axon of AN3 is frequently recorded since its thick and dense axonal arborisations in the lateral protocerebrum of the brain (see Figure 3) are easily accessible. The characteristical tonic response to long lasting stimuli for a broad range of carrier frequencies makes AN3 unique among other ascending neurons and a definite classification due to the physiological properties is possible during intracellular recordings.

Intensity dependence of AN3's response at various frequencies. Example recordings of AN3 show action potentials of clearly axonal origin (Figure 13A).
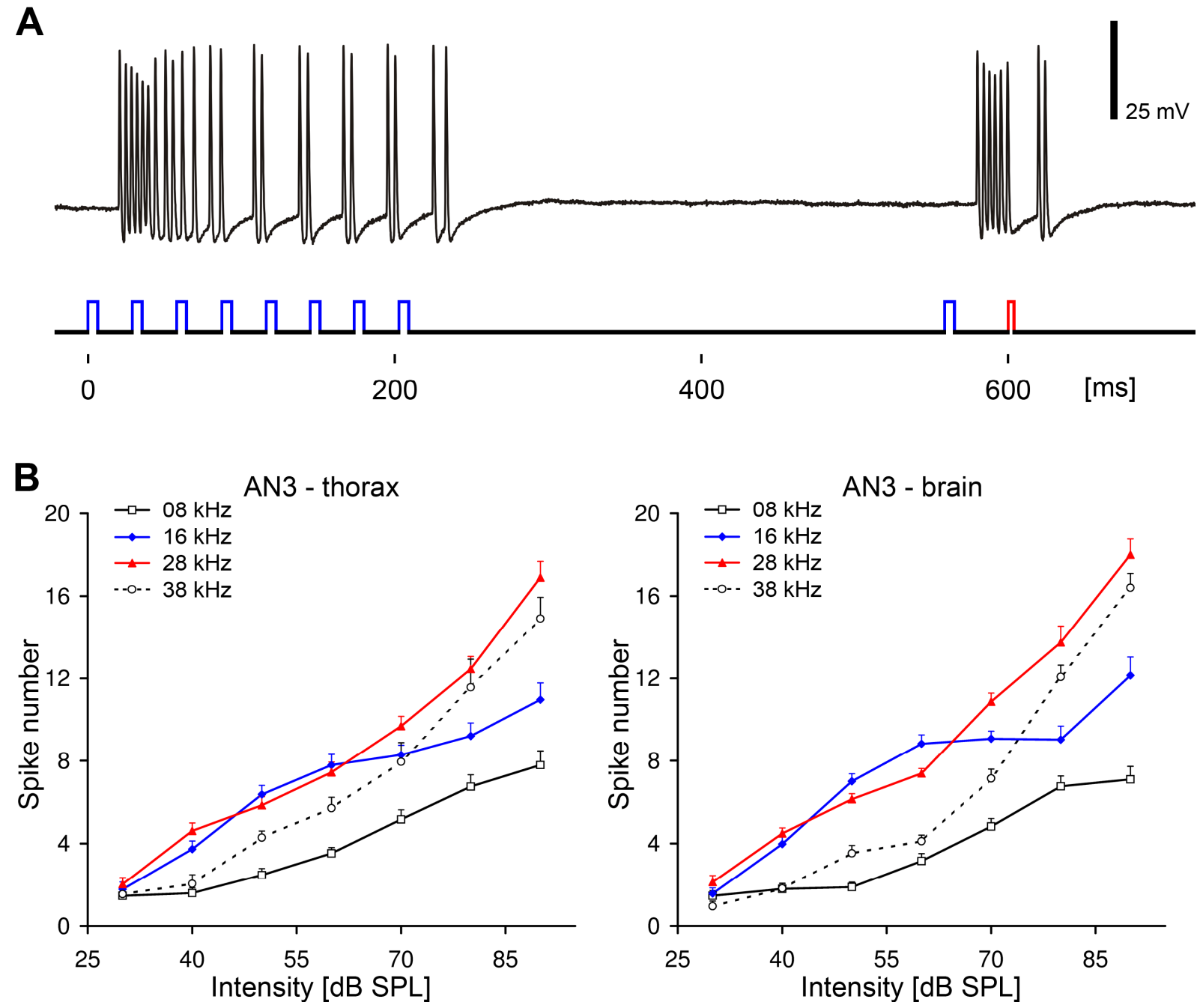

Figure 13: Response of AN3 to acoustic stimuli. Blue colouration - carrier frequency of the male song $(16 \mathrm{kHz})$ and red colouration - carrier of the female reply $(28 \mathrm{kHz})$. A: Example recording of a response to an artificial duet of $A$. nigrovittata (male song at $70 \mathrm{~dB}$ SPL; female answer at $60 \mathrm{~dB}$ SPL; see chapter 2.3.2 for stimulus paradigm). B: Responses of AN3 to standard stimuli (see chapter 2.3.2). Left: Recordings from the prothorax ( $\mathrm{N}=11-12$; data modified from Stumpner \& Molina 2006; means and SE). Right: Recordings from the brain ( $\mathrm{N}=9$; means and $\mathrm{SE}$ ). 
When presenting an artificial duet spikes are elicited to every single pulse independent of carrier frequency. The response to standard stimuli shows gradually increasing spike numbers with increasing intensity at all frequencies tested (Figure 13B). Characteristically, AN3 has a maximum spike number of about 16 to 18 spikes for $90 \mathrm{~dB}$ SPL at ultrasonic frequencies and distinctly lower spike numbers for sonic frequencies. The increasing response for $16 \mathrm{kHz}$ typically attenuates at 60 to $80 \mathrm{~dB}$ SPL and increases again to $90 \mathrm{~dB}$ SPL. All these physiological characteristics obtained from thoracic recordings, including total spike number for a given intensity/frequency, could be confirmed by axonal recordings in the brain. Furthermore, the reported spontaneous activity from thoracic recordings can also be observed in axonal portions of AN3 and, therefore, do not stem from depolarisations due to penetration (Stumpner \& Molina 2006; also compare $30 \mathrm{~dB}$ SPL in Figure 13B left and right).

Responses of AN3 to varying temporal parameters. The spike numbers produced by AN3 steadily decrease with increasing pause durations (Figure 14A left). Therefore, pulses divided by short pauses reveal the strongest response. This applies for the data obtained at thoracic as well as at axonal recording sites. In addition, the total spike number does not differ. A comparison of spike numbers with behavioural data (Figure 14A right) shows no obvious correlation. The increasing response to increasing pulse durations could also be assured by axonal recordings (Figure 14B left). The optimum response of females to the natural pulse duration of the male song cannot be deduced from the response of AN3. However, the responses of AN3 closely copy the varying pulse/pause patterns, which is similar to sensory neurons (Stumpner \& Molina 2006). 

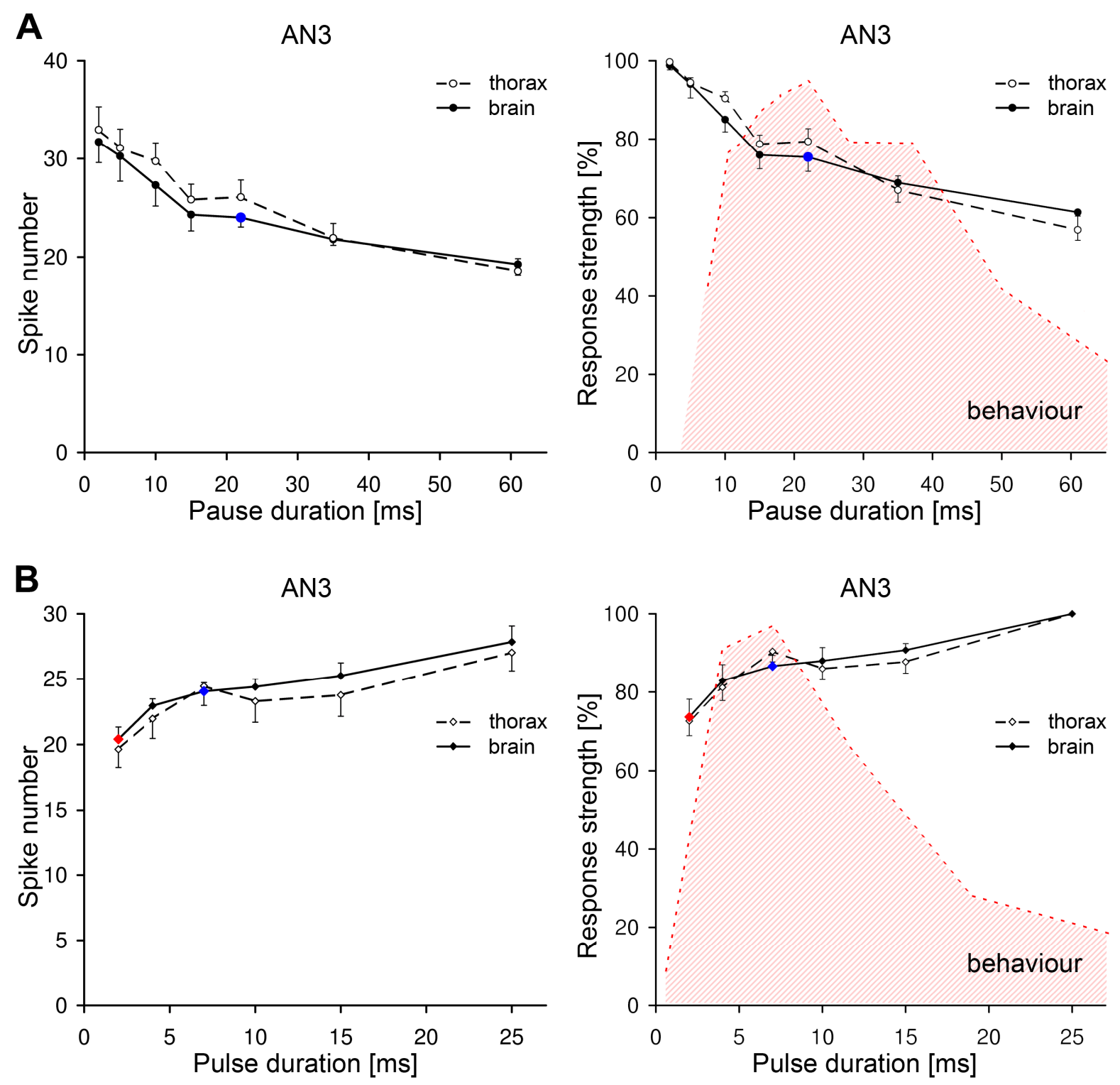

Figure 14: Mean response of AN3 compared in thoracic (dashed lines; data modified from Stumpner \& Molina 2006) and brain (solid lines) recordings to different temporal parameters (white noise; $70 \mathrm{~dB}$ SPL; see chapter 2.3.2 for stimulus paradigms; means and SE). Important natural parameters are emphasised (blue: male song pause/pulse duration; red: female reply pulse duration). Red shaded area indicates female behaviour ( $N=5$, modified from Dobler et al. 1994a). A: Spike number (left) and magnitude of response (right) to stimuli with varying pause duration (thorax: $N=8$; brain: $N=4$ ). B: Spike number (left) and magnitude of response (right) to stimuli with varying pulse duration (thorax: $\mathrm{N}=6$; brain: $\mathrm{N}=4$ ).

Difference in spike latencies. Shortest mean spike latencies of AN3 are found for stimuli at $28 \mathrm{kHz}$ and $70 \mathrm{~dB}$ SPL in thoracic as well as brain recordings. For this carrier frequency the latencies are displayed in Figure 15 for increasing intensities. At $70 \mathrm{~dB}$ SPL the average latency in the thorax is $14.5 \pm 0.9 \mathrm{~ms}(\mathrm{~N}=12$; means and SE) and in the brain $19.2 \pm 0.4 \mathrm{~ms}(\mathrm{~N}=9$; means and SE). Hence, a difference of approximately $4.7 \mathrm{~ms}$ of both recording sites leads to a conduction velocity of $0.9 \pm 0.1 \mathrm{~m} / \mathrm{s}$. This is relatively slow compared to other ascending neurons like AN1 
$(1.2 \pm 0.1 \mathrm{~m} / \mathrm{s}$ - see chapter 3.1 .1$)$. At $50 \mathrm{~dB}$ SPL only a small difference of ca. $3.2 \mathrm{~ms}$ (equivalent to $1.3 \pm 0.1 \mathrm{~m} / \mathrm{s}$ conduction velocity) is obvious which is about the same latency difference as found in AN1. For this intensity, however, the standard error of the thoracic recording is relatively high. Although a similar magnitude of response was observed at very low intensities (30 dB SPL; Figure 13B) the spike latencies were much shorter in thoracic recordings when compared to brain recordings.

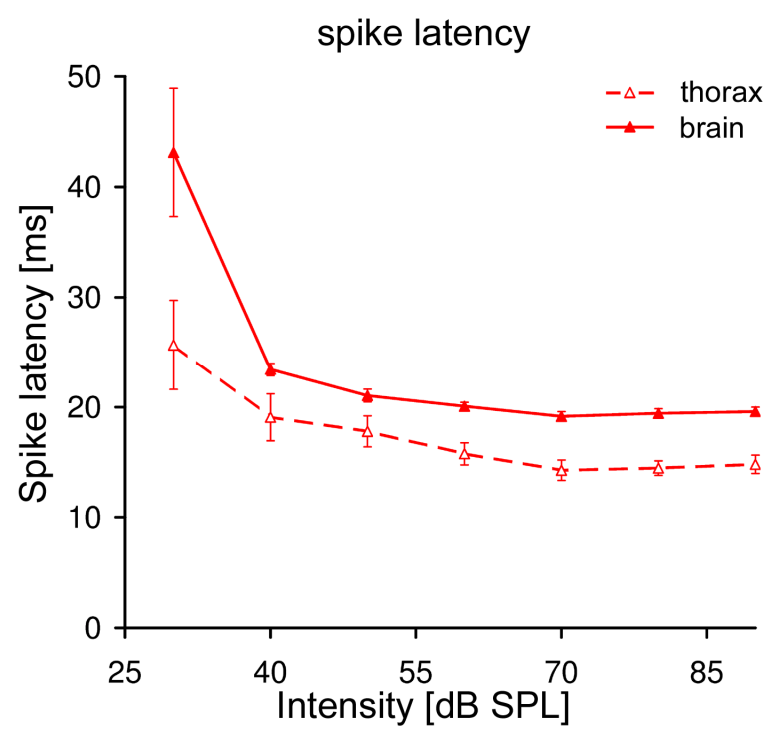

Figure 15: Mean spike latencies of AN3 measured in thoracic and brain recordings at $28 \mathrm{kHz}$ for increasing intensities (thorax: $\mathrm{N}=12$ data from $A$. Stumpner; brain: $\mathrm{N}=9$; means and SE).

\subsubsection{Ascending neuron 5 (AN5-AG7)}

The axonal arborisations of AN5-AG7 (AG7 = abdominal ganglion 7 - where the soma is located; in the following called AN5 for simplicity) in the brain have not frequently been found during the search for sound activated interneurons in the lateral protocerebrum. Due to its activity to intensity response functions with varying carrier frequency, AN5 is similarly variable as the response found in AN2. Only by the strongly reduced activity to the temporal test programs AN5 can be clearly categorised. This reduced response is mainly based on the strict phasic spiking of AN5.

Intensity dependence of AN5's response at various frequencies. When recording intracellularly from AN5 strong bursts of spikes to an artificial duet exclusively occur to the female answer only (Figure 16A). Merely in one recording (out of three) occasionally a spike was elicited to the male song. The intensity 
response functions (Figure 16B) clearly show a low threshold to the female carrier frequency $(28 \mathrm{kHz})$ in both, the thoracic and the brain recordings. The response to $28 \mathrm{kHz}$ has a clear optimum type characteristic in brain recordings, which is not the case when recorded in the thorax. The intraindividual variability is very high for AN5 that, however, does not explain the differences when looking at single AN5 responses.

A
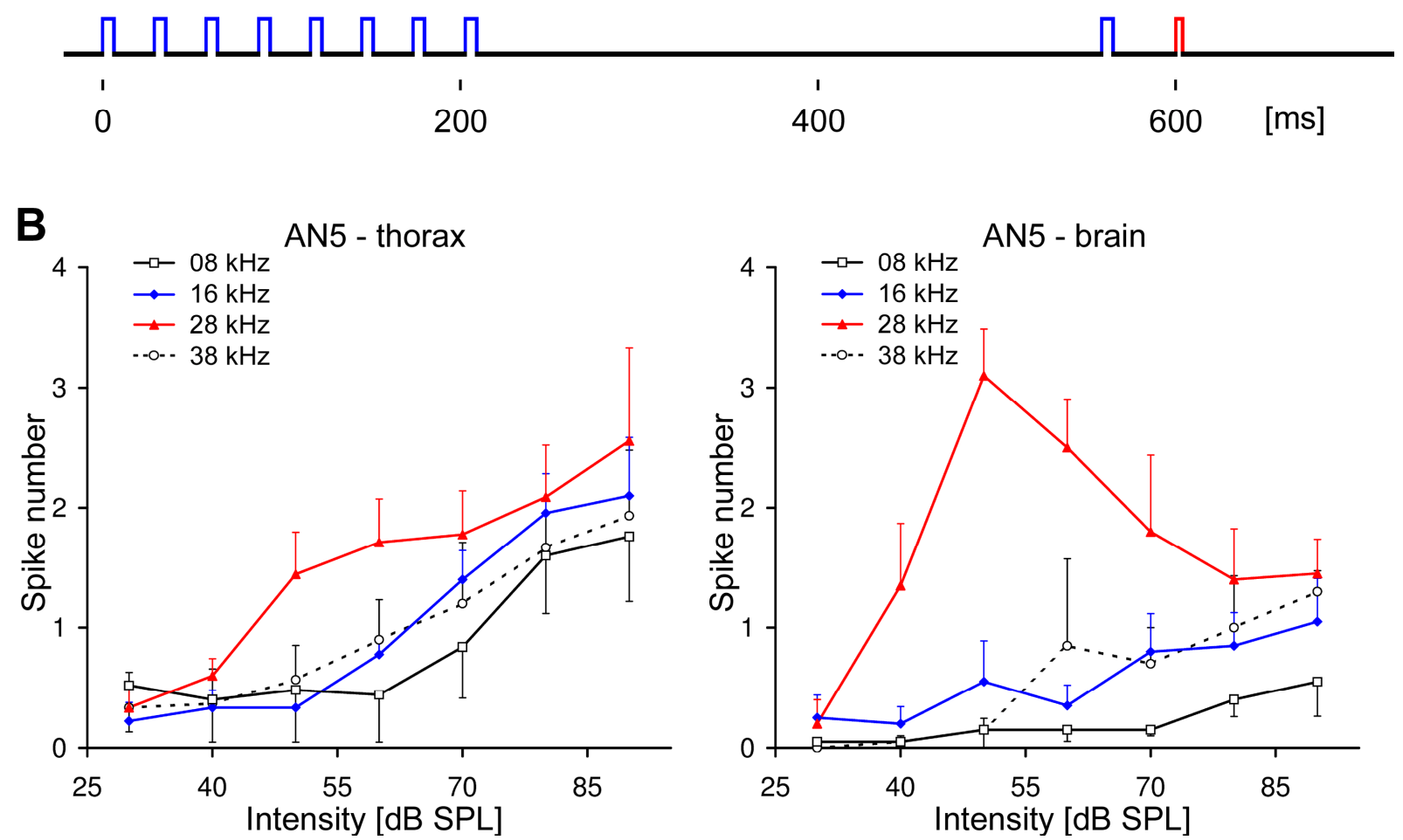

Figure 16: Response of AN5 to acoustic stimuli. Blue colouration - carrier frequency of the male song $(16 \mathrm{kHz})$ and red colouration - carrier of the female reply $(28 \mathrm{kHz})$. A: Example recording of a response to an artificial duet of $A$. nigrovittata (male song at $70 \mathrm{~dB}$ SPL; female answer at $60 \mathrm{~dB}$ SPL; see chapter 2.3.2 for stimulus paradigm). B: Response of AN5 to standard stimuli (see chapter 2.3.2). Left: Recordings from the prothorax ( $\mathrm{N}=9$ for 16 and $28 \mathrm{kHz}, \mathrm{N}=3-6$ for 8 and $38 \mathrm{kHz}$; data recorded by $A$. Stumpner; means and SE). Right: Recordings from the brain ( $\mathrm{N}=4$; means and $\mathrm{SE}$ ).

Response of AN5 to varying pulse duration and changing frequency of the female's reply. The spiking activity to the standard white noise pulse/pause programs is very low. Due to this, no data from thoracic recordings is available that can be compared to axonal recordings in the brain. In the brain, data from two individuals 
display a trend for a preferred response (Figure 17A). The highest activity is found for the shortest pulse duration tested, though, with a high variability. In the natural duet such short pulse durations only occur at the female answer. In Figure 17B the response of AN5 is displayed to variations in carrier frequency of the female reply during an artificial duet. The best spiking activity is at 24 and $28 \mathrm{kHz}$ - the range of the natural female carrier frequency - with $5.5 \pm 1.8$ and $5.0 \pm 2.1$ action potentials (means and SE). The male song frequency $(16 \mathrm{kHz})$ elicits no response.

A

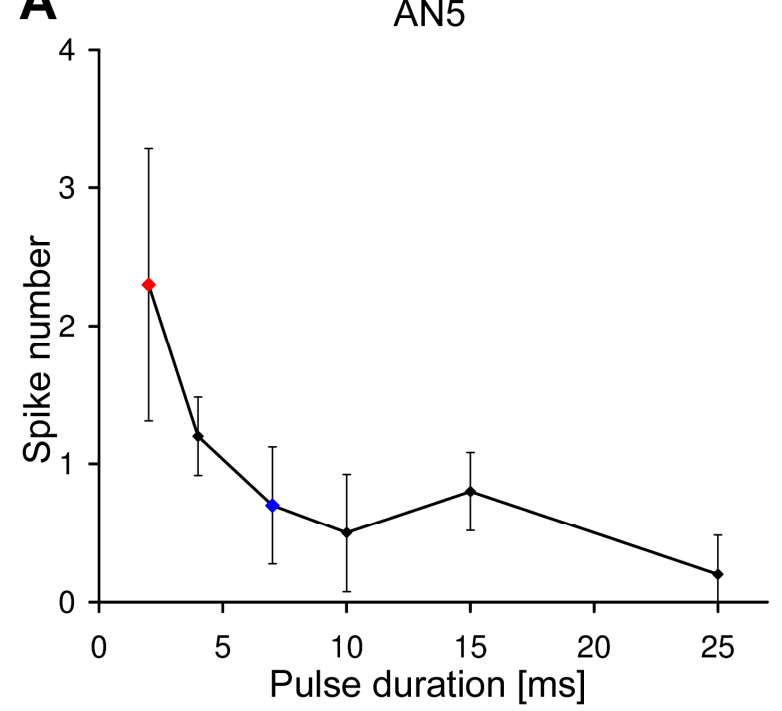

B frequency of the +reply

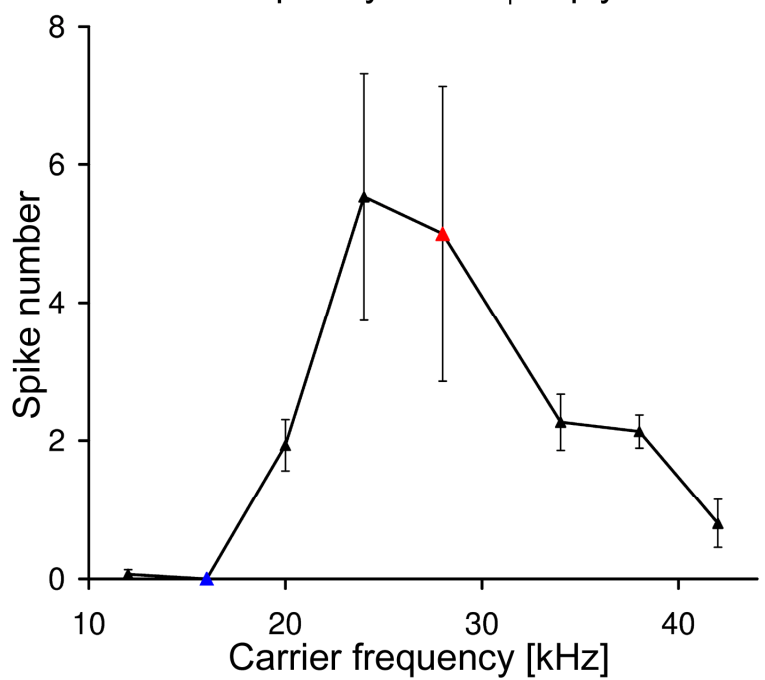

Figure 17: Spike numbers of AN5 to different acoustic stimuli. Blue data point indicates the male's natural carrier frequency and red data point the natural frequency of the female reply (see chapter 2.3.2 for stimulus paradigms). A: Spike number with standard deviation to stimuli with varying pulse duration (brain: $\mathrm{N}=2$ ). $\mathbf{B}$ : Mean response to the female answer with varying carrier frequency in an artificial duet. Data only shown for brain recordings $(\mathrm{N}=3$; means and $\mathrm{SE})$.

Difference in spike latencies. Spike latencies of $18.3 \pm 0.8 \mathrm{~ms}(\mathrm{~N}=9$; mean and SE) were measured in the thorax at $28 \mathrm{kHz}$ and $90 \mathrm{~dB} \mathrm{SPL}$. In the brain the latency of the action potentials is $20.5 \pm 2.1 \mathrm{~ms}(\mathrm{~N}=4$; mean and $\mathrm{SE}$ ). Thus, the conduction velocity for spikes in AN5 accounts approximately $1.9 \pm 0.1 \mathrm{~m} / \mathrm{s}$. This result is higher than the velocity of $1.3 \mathrm{~m} / \mathrm{s}$ measured from the thorax to the abdomen (Stumpner 1999a). This difference in conduction velocity may be correlated to the considerable smaller diameter of the axon in the abdominal ganglia when compared to the diameter of the axon from the neck connective to the brain (Stumpner 1999a). Nevertheless, AN5 seems to have the fastest conduction velocity among all other ascending neuron. 


\subsubsection{T-shaped neuron 1 (TN1)}

Another example of a frequently recorded interneuron in the brain is TN1. The characteristical more or less broad banded phasic response to long stimulus durations (e.g. $50 \mathrm{~ms}$ ) in combination with a narrow banded response to 24 and $28 \mathrm{kHz}$ when pulse durations are short (e.g. $2 \mathrm{~ms}$ ), allows an easy identification of TN1 during the recording procedure.

Confirmation of morphology. In Figure 18 a comparison of the morphology of two different TN1s (left - stained during a brain recording; right - stained during a thoracic recording) are displayed. Clearly, one can see the similarity of branching pattern in the proto- and deutocerebrum. Although two major collaterals of the axon are found in the left drawing (only one in the right drawing) the overall extent of brain regions innervated is similar. The strength of single collaterals of TN1 may be pronounced to a greater or lesser extent in different individuals. These variations seem to be common and have also been observed for AN1 (personal communication A. Stumpner and own observations).
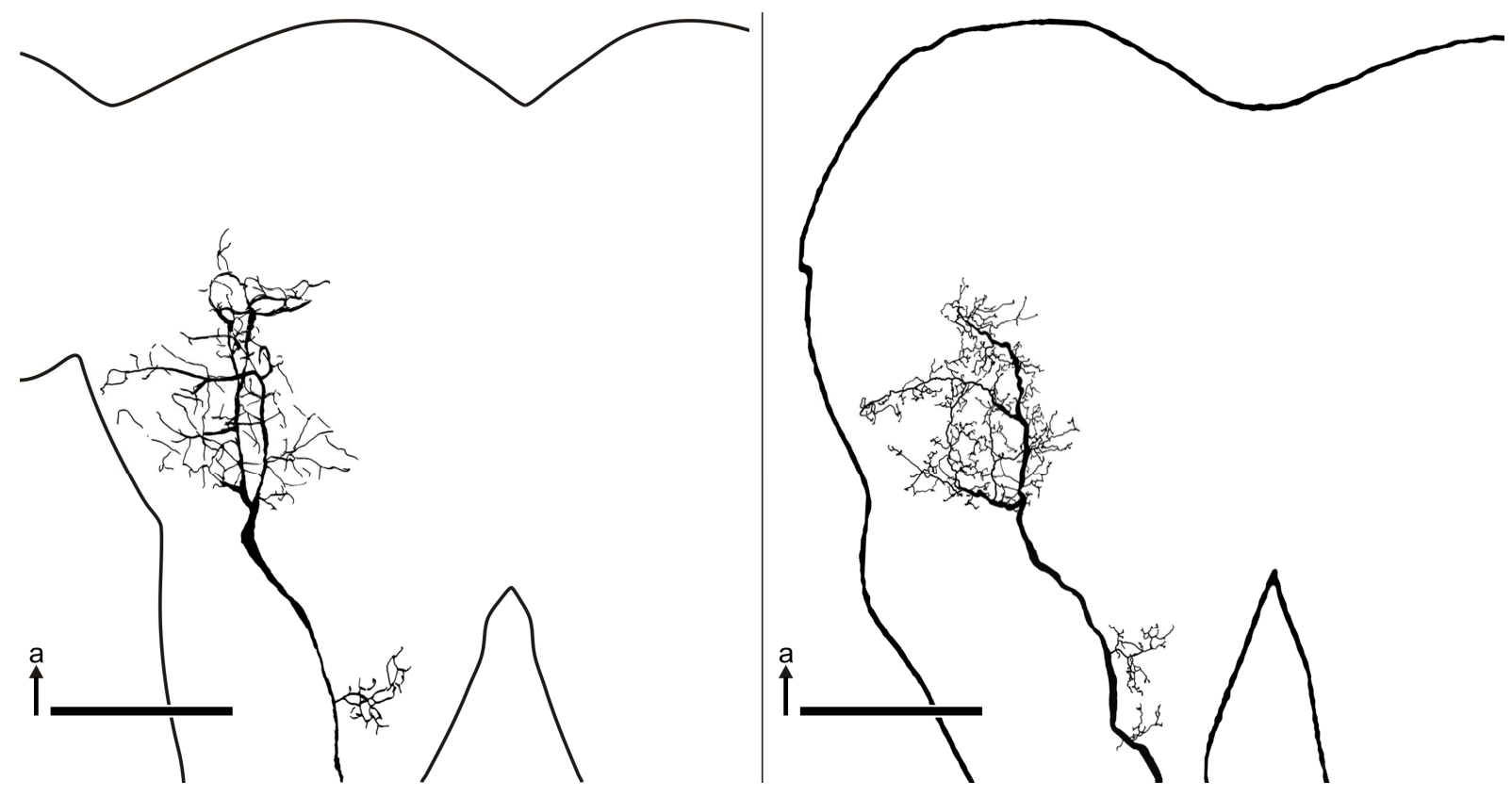

Figure 18: Morphology of TN1s in two brains of $A$. nigrovittata. Bar represents $200 \mu \mathrm{m}$. Left: Drawing of TN1's arborisations in the brain from a staining done in the brain. Right: Drawing of TN1's brain arborisations according to a staining done in the thorax (modified after Stumpner 1999a). $a=$ anterior. 
Intensity dependence of TN1's response at various frequencies. Intracellular recordings from TN1 reveal a strong spiking activity to the female reply (28 kHz) when presenting an artificial duet (Figure 19A). To the male song at $16 \mathrm{kHz}$ the responses can be very variable. In some recordings no action potentials are elicited while in others strong responses (although not as strong as to the female reply) can be observed.

A

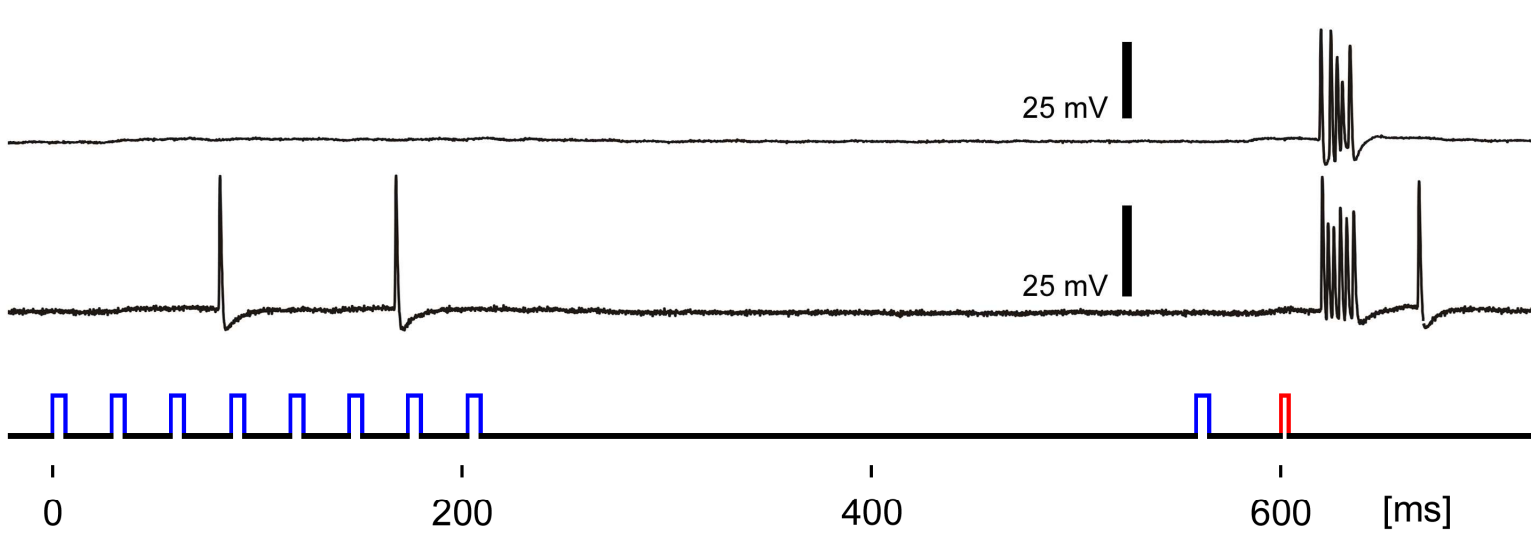

B
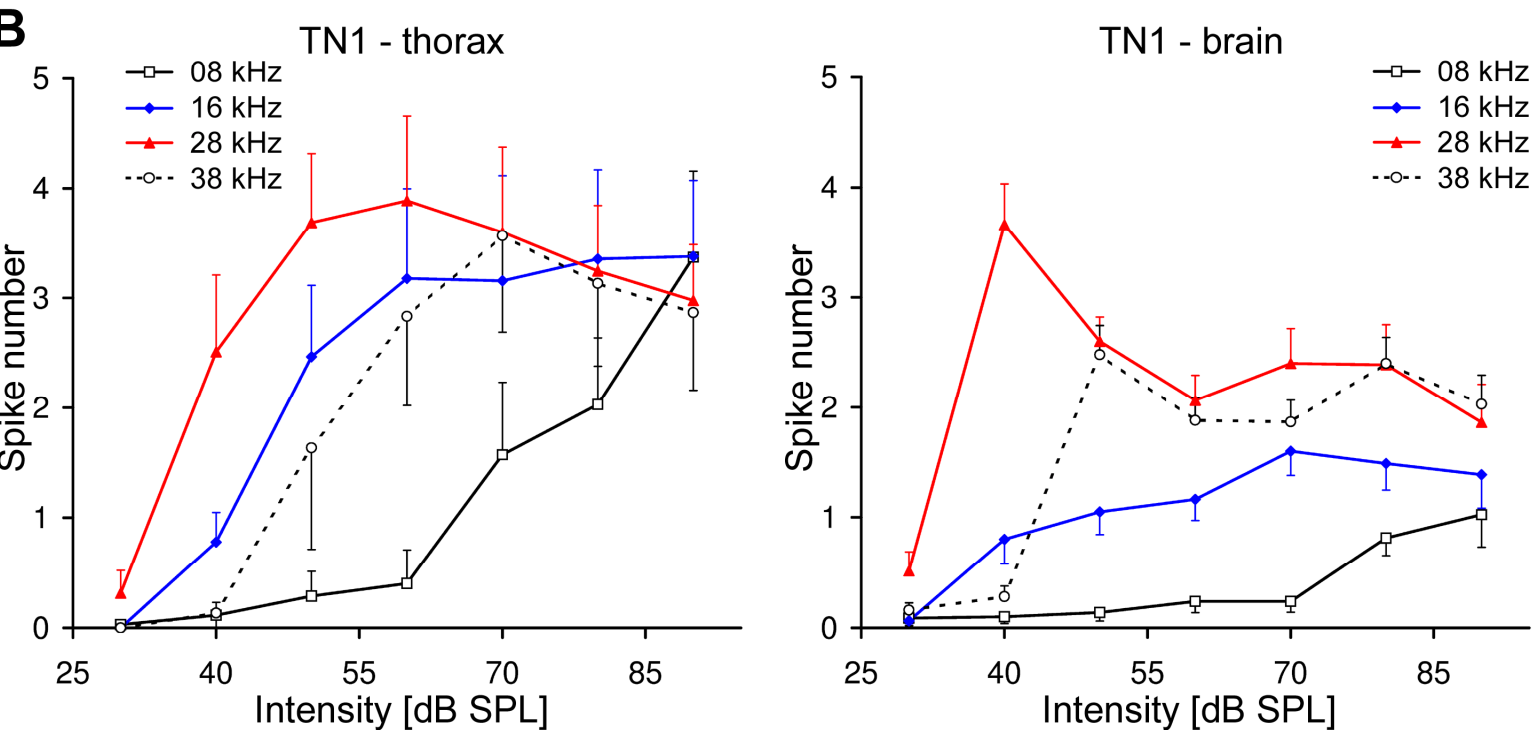

Figure 19: Response of TN1 to acoustic stimuli. Blue colouration - carrier frequency of the male song $(16 \mathrm{kHz})$ and red colouration - carrier of the female reply $(28 \mathrm{kHz})$. A: Example recording of two different TN1 responses to an artificial duet of $A$. nigrovittata (male song at $70 \mathrm{~dB}$ SPL; female answer at $60 \mathrm{~dB}$ SPL; see chapter 2.3.2 for stimulus paradigm). B: Responses of TN1 to standard stimuli (see chapter 2.3.2). Left: Recordings from the prothorax ( $\mathrm{N}=6-9$; data modified from Stumpner \& Molina 2006; means and SE). Right: Recordings from the brain ( $\mathrm{N}=15-16$; means and $\mathrm{SE})$.

A stimulation with standard stimuli elicited spiking in TN1 also for $16 \mathrm{kHz}$ stimuli at low intensities (Figure 19B). Nevertheless, stimulation with $28 \mathrm{kHz}$ shows a comparatively lower threshold. Large differences of the overall response characteristic of TN1 can be observed between thoracic and brain recordings. While 
the average response for $28 \mathrm{kHz}$ exhibits an optimum type curve with the strongest activity at 50 to $70 \mathrm{~dB} \mathrm{SPL}$ in thoracic recordings, strongest spiking in brain recordings on average was observed at $40 \mathrm{~dB} S P L$ only. This difference of response characteristics also applies for $38 \mathrm{kHz}$ (although shifted to $10 \mathrm{~dB}$ higher intensities at both recording sites). 16 and $38 \mathrm{kHz}$ stimuli evoke similar spike numbers in thoracic recordings. In the brain, the response to $16 \mathrm{kHz}$ is consistently lower (except for $40 \mathrm{~dB} \mathrm{SPL}$ ) than the response to ultrasonic frequencies. At $8 \mathrm{kHz}$ the spiking activity is lowest at both recording sites although higher spike numbers are obtained in the thorax.

When looking at the spiking activity to $28 \mathrm{kHz}$ stimuli, responses of single TN1s recorded in the thorax show similar intensity dependency as found in recordings of the axonal recording site (compare highlighted graphs in Figure 20 left to the single responses in Figure 20 right). The variability of both recording sites is high compared to other ascending neurons (high variability also reported for TN1 in Stumpner \& Molina 2006) but spike numbers up to 7-8 at medium intensities are only found in thoracic recordings (marked by an asterisk in Figure 20 left). In the brain recordings a maximum of response is mostly reached at $40 \mathrm{~dB}$ SPL. For higher intensities, spike numbers decrease in most specimens.

TN1 - thorax

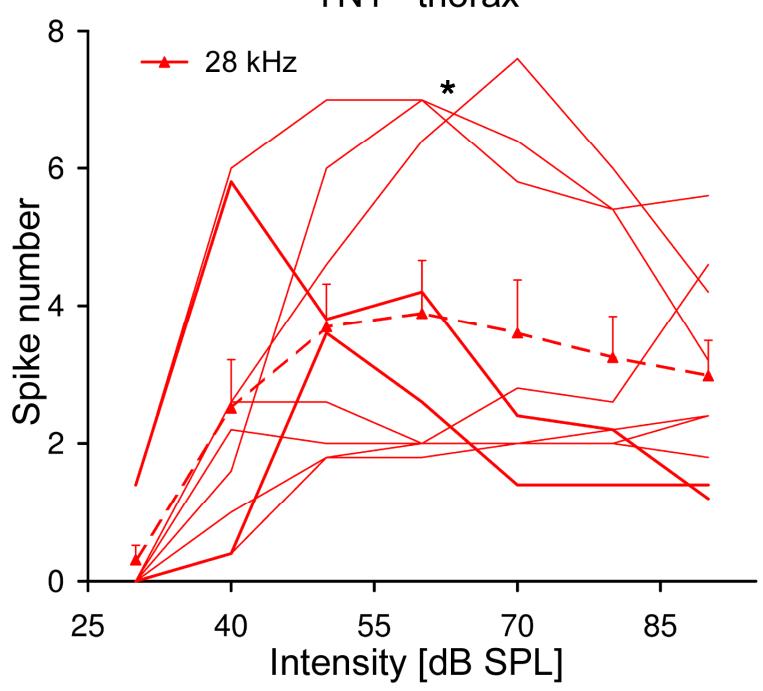

TN1 - brain

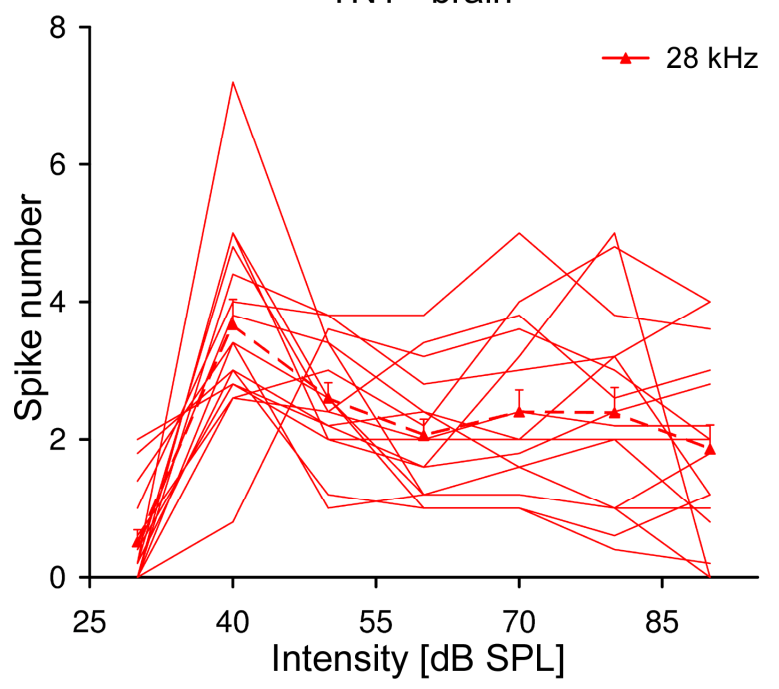

Figure 20: Mean (dashed lines with SE) and single (solid lines) responses of TN1 to $28 \mathrm{kHz}$ and increasing intensities (50 ms stimuli) recorded in the thorax (left, $\mathrm{N}=9$; data taken from Stumpner \& Molina 2006) and in the brain (right, $\mathrm{N}=16$ ). Two single responses from the thoracic recording site are very similar to axonal recordings and are highlighted. 3 single responses of TN1 recorded in the thorax showed high spike numbers at medium intensities (marked by an asterisk). This was never found in brain recordings. 
The differences found in the average responses collected in the two recording sites are pronounced to the greatest extend among all ascending neurons and one might wonder, whether the data even might originate from two different interneurons. Characteristics like the response to the female reply only in a natural duet and the maximum spike number (of ca. 3-4) to $28 \mathrm{kHz}$ at low intensities might suggest AN5 to be the appropriate candidate for the data of the brain recordings presented (see Figure 16). However, a definite morphological affirmation of typical TN1 arborisation sites in the brain could be made for 3 cases in addition to the physiological data.

Further, to a staining of the brain terminations also a staining of the soma in the typical region known for TN1 in the prothoracic ganglion was achieved in one case (Figure 21). This finding excludes AN5 to be the source of the physiological data since its soma is situated in the $7^{\text {th }}$ abdominal ganglion. Unfortunately, the dense dendritic branching and the descending axon of TN1 could not be detected, which was most likely due to the long distance from the penetration site in the brain. But the morphological data obtained show a clear correlation with TN1 known from thoracic recordings.

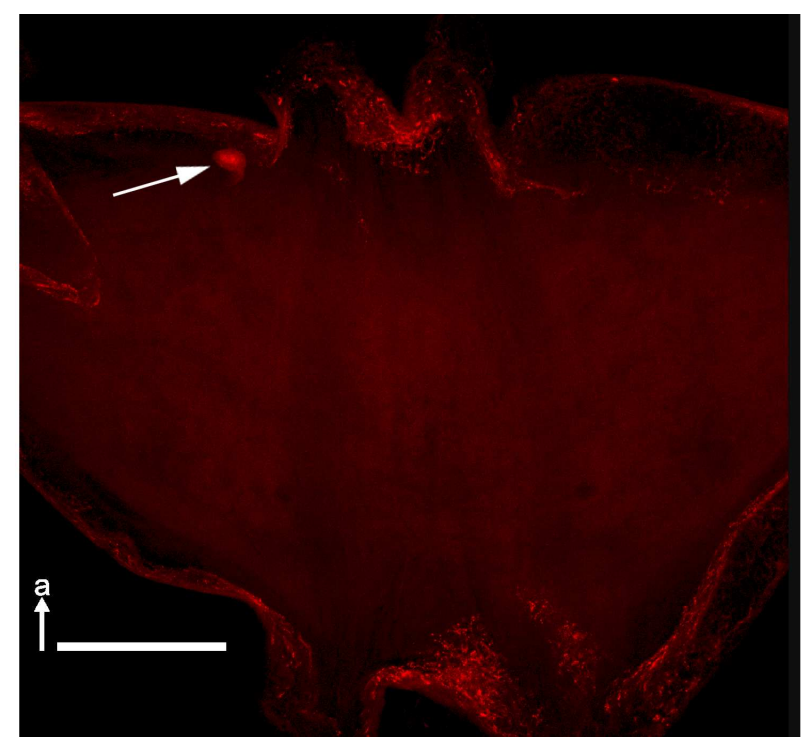

Figure 21: Prothoracic ganglion of A. nigrovittata with a Cy3-labelled soma of TN1 (marked by the white arrow). The retrograde staining was performed by iontophoretic application of a fluorescent dye in the brain. Bar represents $150 \mu \mathrm{m}$. $\mathrm{a}=$ anterior.

In Figure 22 the relative differences of TN1's spiking activity to ipsilateral and contralateral stimulation (relative to the side of penetration) are displayed for brain and thoracic recordings. The maximum difference in spiking activity can be observed for $28 \mathrm{kHz}$ at $40 \mathrm{~dB} \mathrm{SPL}$ in the brain recordings of TN1 (Figure 22A). This high degree of directionality at low intensities can only be found for TN1 in the thoracic recordings (highest degree of directionality among all ascending neurons Figure 22B). Similarly to the data from the thorax, also for $16 \mathrm{kHz}$ stimuli highest 
values can be seen. The overall directionality characteristics to increasing intensities, however, differ to a minor extent at $16 \mathrm{kHz}$ and to a great extent for $28 \mathrm{kHz}$, when comparing both recording sites. The main reason for this may be the difference in spike numbers at the corresponding intensities (see Figure 19B). Nevertheless, high degrees of directionality are ascribed to central enhancement (Molina \& Stumpner 2005) in addition to biophysical differences (e.g. sound shadow). A relative difference above $70 \%$ for $28 \mathrm{kHz}$ at low intensities has only been found in TN1, which therefore serves as an additional corroboration of physiological identification of TN1 in brain recordings.
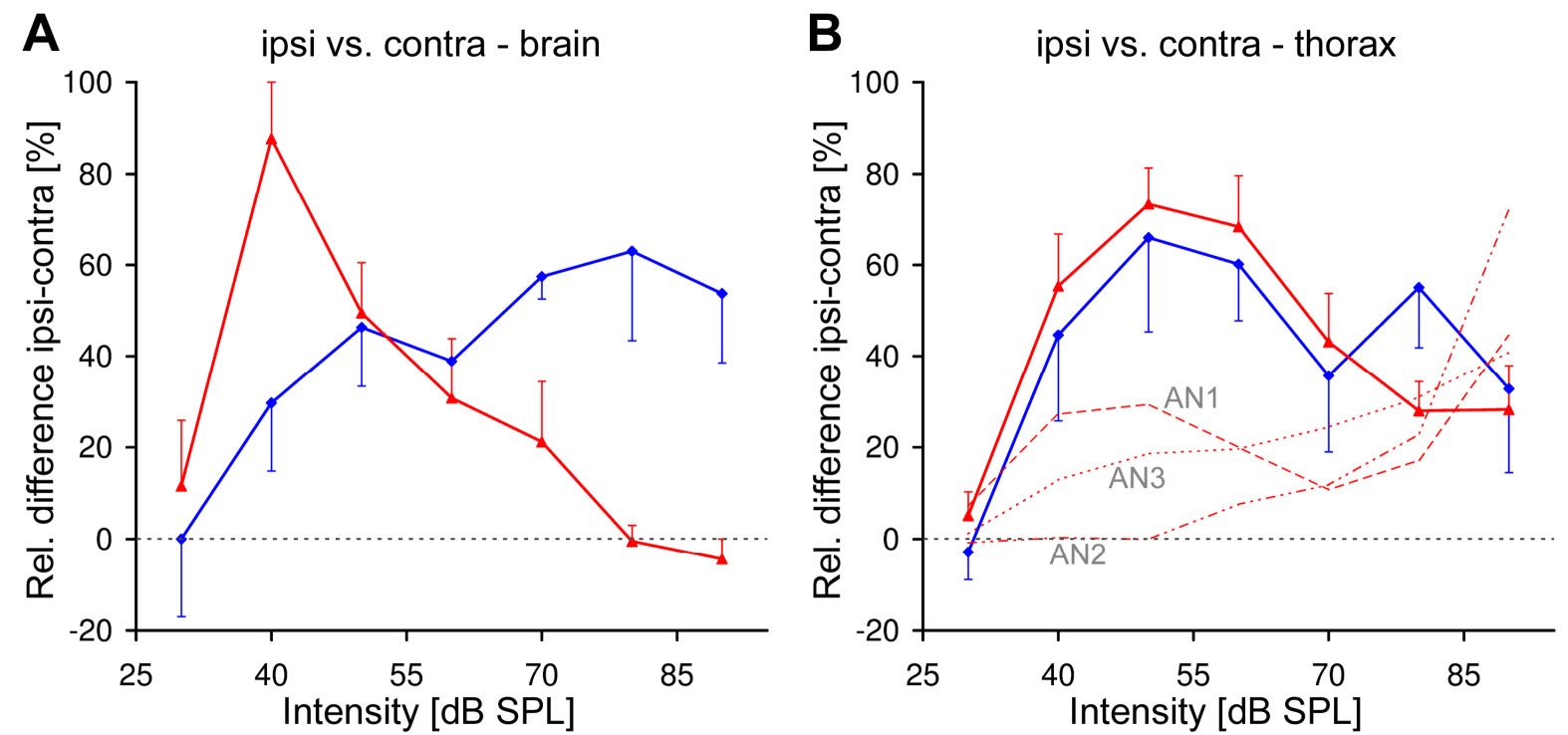

Figure 22: Normalised difference of TN1's responses for 16 (blue) and $28 \mathrm{kHz}$ (red) at increasing intensities to ipsi- and contralateral stimulation (relative to the side of penetration). A: Data of TN1 obtain in brain recordings ( $\mathrm{N}=3$; means and $\mathrm{SE}$ ). $\mathrm{B}$ : Data obtained for TN1 in thoracic recordings. For comparison also the normalised differences of AN1, AN2 and AN3's responses at $28 \mathrm{kHz}$ are displayed ( $\mathrm{N}=4-6$ for $\mathrm{TN} 1$, means and $\mathrm{SE} ; \mathrm{N}=5$ for $\mathrm{AN} 1$, mean; $\mathrm{N}=10$ for $\mathrm{AN2}$, mean; $\mathrm{N}=13$ for $\mathrm{AN} 3$, mean; data taken from Stumpner \& Molina 2006). 100\% means that the maximum spike response of the corresponding frequency occurred exclusively to ipsilateral stimulation $(-100 \%$ would be the opposite).

Response of TN1 to varying temporal parameters. In Figure 23 the responses of TN1 to variations of temporal parameters are displayed. The spike number of both, responses to varying pause and varying pulse duration is higher when recorded in the thorax close to the dendritic input than when recorded in the brain. The relative dependence on the various parameters, however, is similar in both preparations. 
A

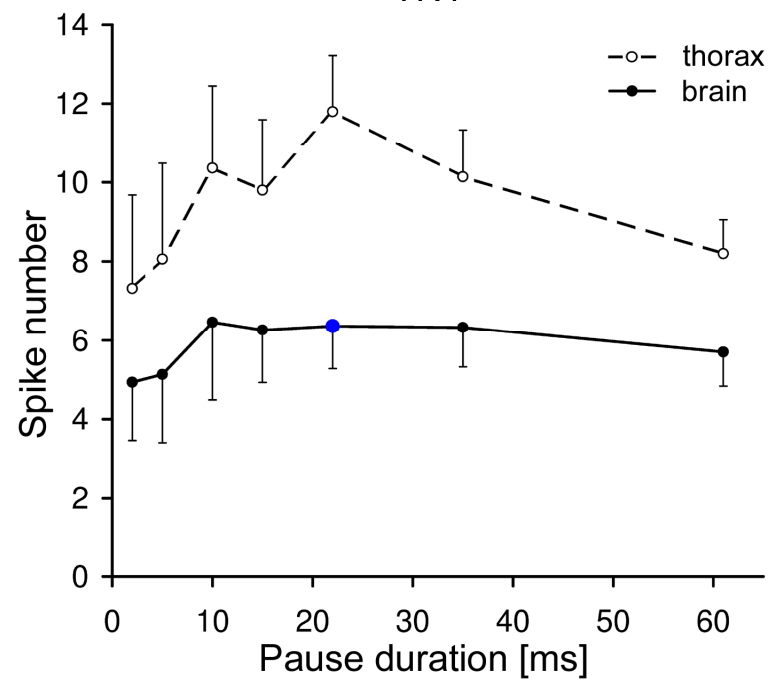

B

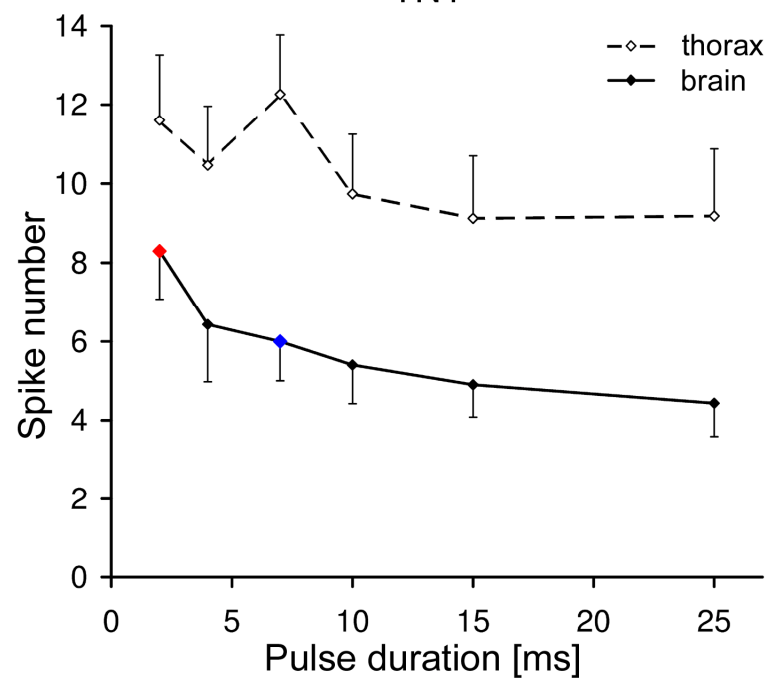

TN1

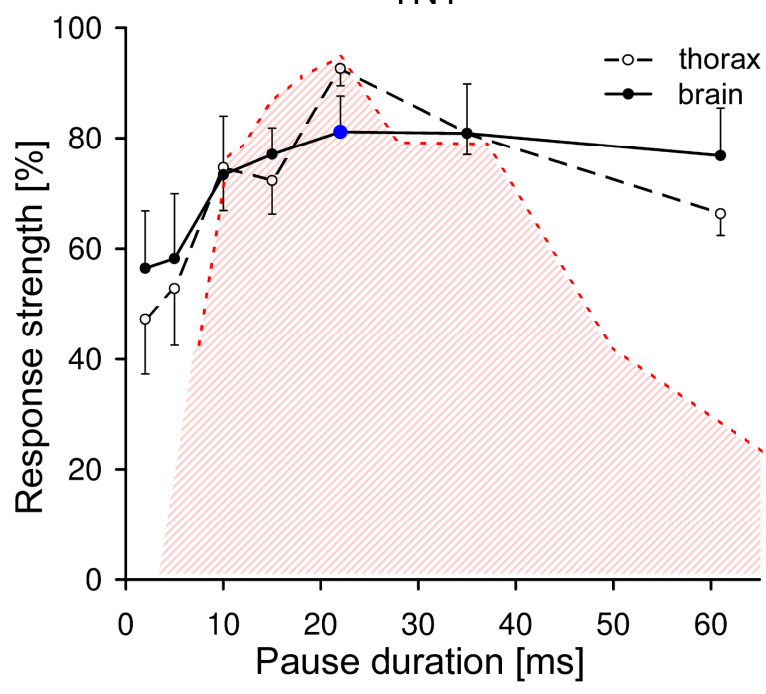

TN1

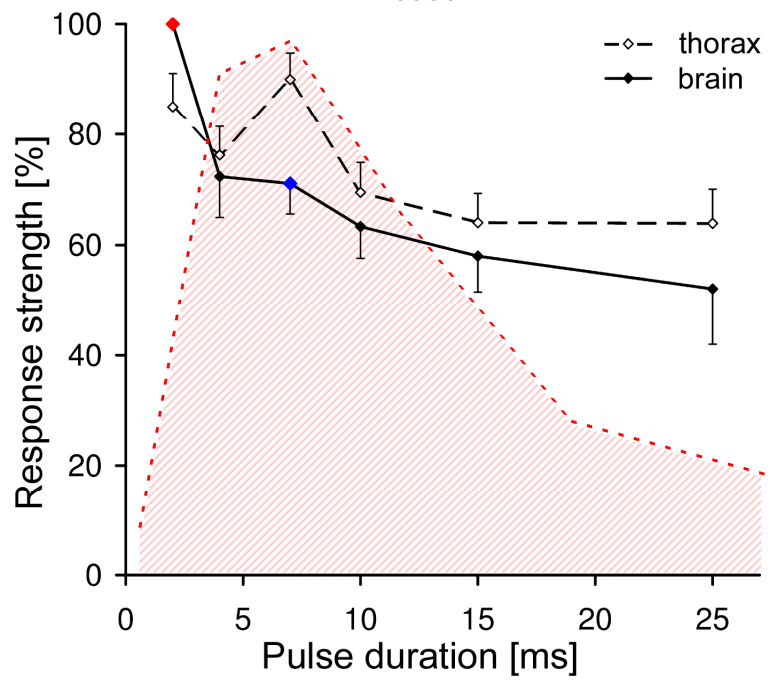

Figure 23: Mean responses of TN1 compared in thoracic (dashed lines; data modified from Stumpner \& Molina 2006) and brain (solid lines) recordings to different temporal parameters (white noise; $70 \mathrm{~dB}$ SPL; see chapter 2.3.2 for stimulus paradigm; means and SE). Important natural parameters are emphasised (blue: male song pause/pulse duration; red: female reply pulse duration). Red dashed line indicates female behaviour ( $\mathrm{N}=5$, modified from Dobler et al. 1994a). A: Spike number (left) and magnitude of response (right) to stimuli with varying pause duration (thorax: $N=10$; brain: $N=6$ ). B: Spike number (left) and magnitude of response (right) to stimuli with varying pulse duration (thorax: $\mathrm{N}=10$; brain: $\mathrm{N}=6$ ).

The responses of TN1 with varying pause durations are nearly independent from pause duration between 22 and $61 \mathrm{~ms}$. The relative spike numbers, however, only show a slight correlation to the female behaviour at pause durations shorter than $22 \mathrm{~ms}$ (Figure 23A right). For varying pulse durations TN1's spiking activity is best at very short pulses (2 ms, Figure 23B left), but no correlation to the female behaviour can be observed (Figure 23B right). 
Response to different frequencies of the female answer in an artificial duet. Since TN1's highest spiking activity is found at short pulse durations, it can be expected that a presentation of a complete duet would elicit the strongest response to the female answer. When varying the carrier frequency of the female reply the response of $\mathrm{TN} 1$ exhibits band-pass filter characteristics with a maximum response at 24 and $28 \mathrm{kHz}$ (Figure 24). Similar results were obtained for AN2 and AN5 (see Figure 11 and 17B).

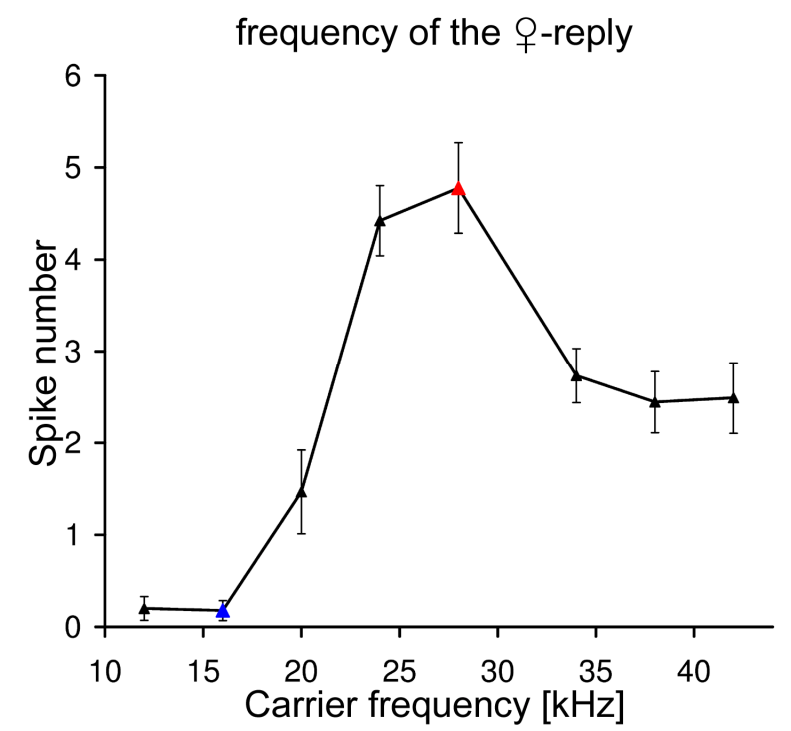

Figure 24: Mean response to the female answer with varying carrier frequency in an artificial duet (see chapter 2.3.2 for stimulus paradigm; $\mathrm{N}=9$; means and SE). Blue data point indicates the male's natural carrier frequency and red data point the natural frequency of the female reply.

Difference in spike latencies. Best responses of TN1 to $50 \mathrm{~ms}$ stimuli for $28 \mathrm{kHz}$ are found at different intensities when recorded in the thorax or in the brain, thus, also shortest latencies can only be found at differing intensities. The shortest mean latency is $16.5 \pm 1.0 \mathrm{~ms}$ (for $90 \mathrm{~dB} \mathrm{SPL} ; 28 \mathrm{kHz}$; $\mathrm{N}=9$; mean and $\mathrm{SE}$ ) for thoracic recordings and $19.5 \pm 0.6 \mathrm{~ms}$ (70 dB SPL; $28 \mathrm{kHz} ; \mathrm{N}=15$; mean and $\mathrm{SE}$ ) for brain recordings. This accounts for a conduction velocity of $1.4 \pm 0.1 \mathrm{~m} / \mathrm{s}$. The spike latencies of brain recording at $90 \mathrm{~dB} S P L$ are $21.2 \pm 1.9 \mathrm{~ms}(\mathrm{~N}=15$; mean and $\mathrm{SE})$ and therewith comparatively longer than at $70 \mathrm{~dB}$ SPL.

Secondary auditory input into TN1 within the brain. In brain recordings of TN1 axonal spikes can be seen that are subsequently followed by a graded potential during auditory stimulation (Figure 25). These graded potentials also occur when TN1 produced no action potentials to a stimulus (marked by an arrow in Figure 25A). Therefore, the origin of the graded potentials is neither a stronger form of repolarisation caused by an action potential, nor a self-induced potential via a feedback loop, but the input from a different neuron within the brain instead. 


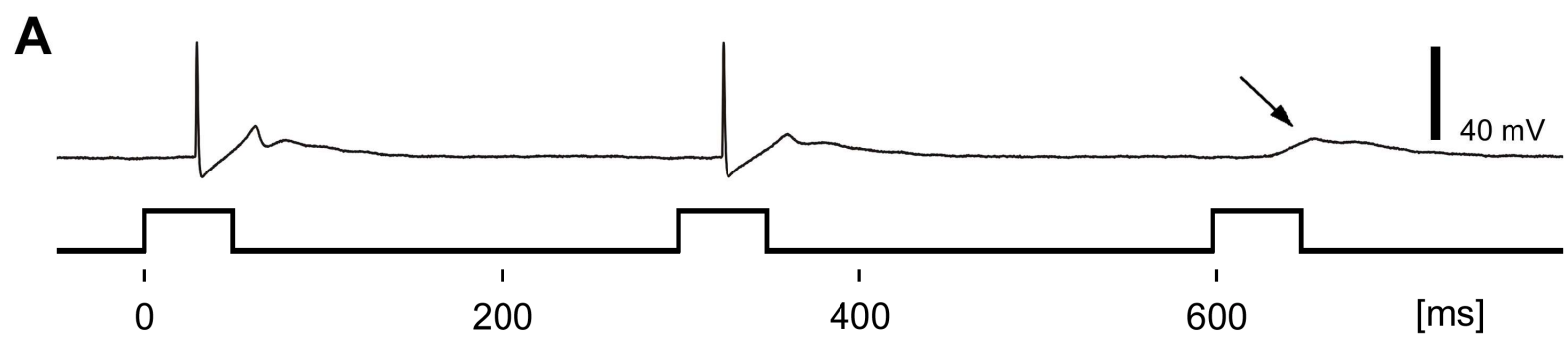

B

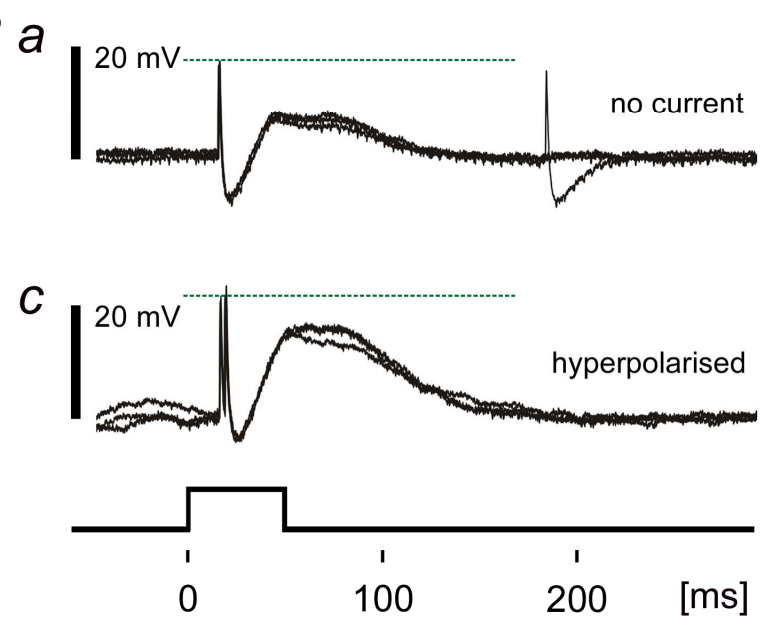

$b$
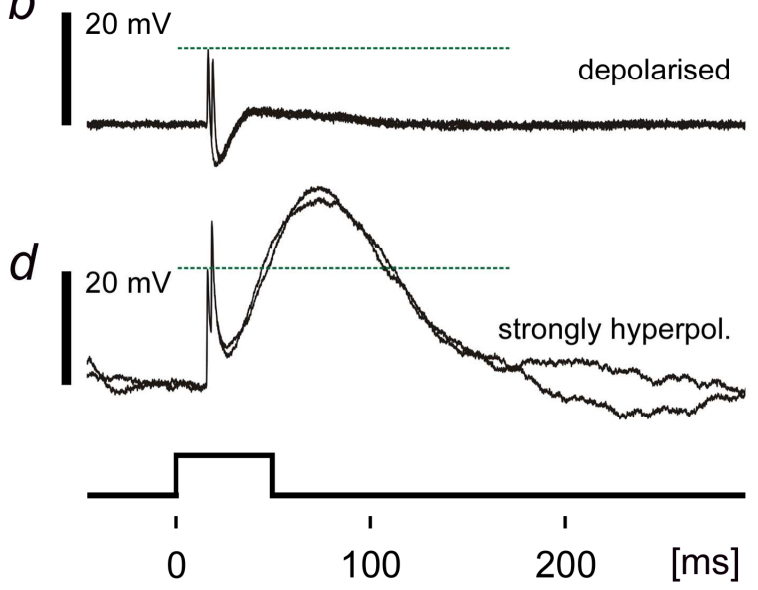

Figure 25: Example recordings of TN1 in axonal portions within the brain. A: Action potentials with a subsequent graded potential to $50 \mathrm{~ms}$ stimuli $(90 \mathrm{~dB} \mathrm{SPL}$ and $28 \mathrm{kHz}$ ). The arrow marks a graded potential that occurred, when no spike was produced to the stimulus. B: Overlay of three sample traces (d: only two) obtained in the same individual for $50 \mathrm{~ms}$ stimuli at $90 \mathrm{~dB}$ SPL and $28 \mathrm{kHz}$. Green lines mark the amplitude of the first spike. $a$ : Recordings without any current induction. $b$ : Recordings with a depolarising current (ca. $+0.5 \mathrm{nA})$. c: Recordings with a hyperpolarising current (ca. $-0.5 \mathrm{nA})$. $d$ : Recordings with a strong hyperpolarising current (ca. $-1 \mathrm{nA}$ ). Note that no extra spikes are elicited, but the amplitude of the graded potential is affected.

During intracellular brain recordings these graded potentials are a characteristical feature of TN1. They were seen in every single recording, which was not the case in any other ascending neuron. The amplitude of the graded potential, however, can differ to a great extend between individuals. In some recordings it reached half the amplitude of a spike (Figure 25Ba) and in others the potentials were barely seen. Injection of a depolarising current while recording TN1's axon can lower the amplitude of the graded potential and of the action potential (Figure 25B $b$ ). The reversal potential of the graded potential, however, could not be detected, since the resistances of the capillaries used did not allow the injection of enough current for reaching the reversal potential of the graded depolarisation. Hyperpolarisation strongly increased the amplitude of the graded potential (Figure 25Bc,d), which may become even higher than an action potential without eliciting extra spikes. 
Origin of the graded potential in the axon of TN1. In Figure 26A the responses of two different TN1s to $500 \mathrm{~ms}$ stimuli (white noise; $70 \mathrm{~dB}$ SPL) are displayed. The graded potential within TN1 can be clearly seen throughout the whole length of the stimulus which is an indication that AN3 is the source of this potential, since no other ascending neuron shows a tonic response to this stimulus.
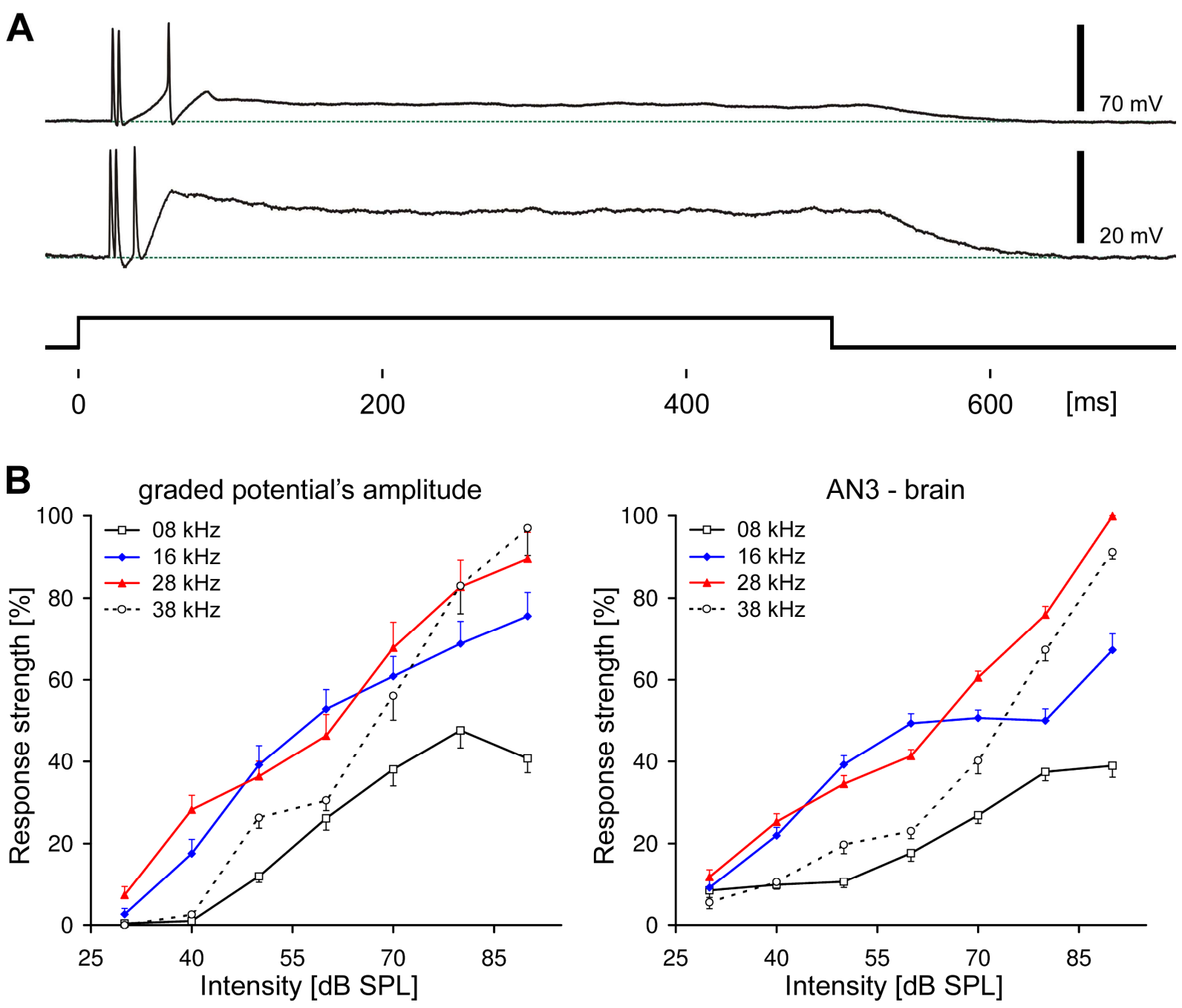

Figure 26: A: Example recordings from two individuals in the axon of TN1 to a 500 ms stimulus (white noise at $70 \mathrm{~dB}$ SPL). Green dotted lines indicate the resting membrane potential. B: Response strength of TN1's graded potential (left, $\mathrm{N}=5$; mean and SE) and AN3 (right, $\mathrm{N}=9$; mean and SE) to standard stimuli (see chapter 2.3.2). Blue colouration - carrier frequency of the male's song $(16 \mathrm{kHz})$ and red colouration - carrier of the female's reply $(28 \mathrm{kHz})$.

Assuming a close correlation of the potential's amplitude and spiking activity of the presynaptic neuron would uncover the possible candidate for this input to TN1. In Figure 26B (left) relative amplitudes of the graded potential are plotted against increasing intensities of the standard frequencies. At 50 and $60 \mathrm{~dB}$ SPL the response to $16 \mathrm{kHz}$ is slightly higher than to $28 \mathrm{kHz}$. At very high intensities ( $90 \mathrm{~dB} \mathrm{SPL}$ ) the response strength is highest for ultrasonic frequencies, whereas the response to 
$8 \mathrm{kHz}$ is only half the maximum. Very similar responses can be found in the spiking of AN3 (Figure 26B right). However, the latency of the graded potential is $29.3 \pm 1.1 \mathrm{~ms}$ $(\mathrm{N}=9$; measured only when no action potential concealed the beginning of the graded potential; mean and SE) and indicates that AN3 (latency of $19.2 \mathrm{~ms}$ ) is not directly connected to TN1. At least one (or even more) local interneuron(s) within the brain must mediate the graded input to TN1.

The effect of the graded potential in TN1. When an action potential from TN1 (generated in the prothorax) simultaneously occurs with a graded potential (generated in the brain), the amplitude of the spike can be higher (at least in some recordings) than the amplitude of a spike in the absence of the graded potential (Figure 27 left). This summation has the greatest effect on the spike's amplitude when the action potential sits directly on the maximum of the graded potential. Spikes sitting outside the maximum experience a smaller summation (Figure 27 middle marked by an arrow). The result of this summation may be an increased transmitter release at the presynapse. This effect could only be measured in recordings where the amplitude of the graded potential was large. Whether this effect may also occur in other TN1s can not be decided from the data obtained so far. A reduction of spike amplitude, on the other hand, when sitting on top of a graded potential was never observed. The pronounced repolarisation phase of an action potential causes a nearly complete shunting of the graded potential's amplitude to the level of the resting membrane potential (Figure 27). If another spike occurs exactly during this repolarisation phase the amplitude will not be increased (Figure 27 right - indicated by an arrow).
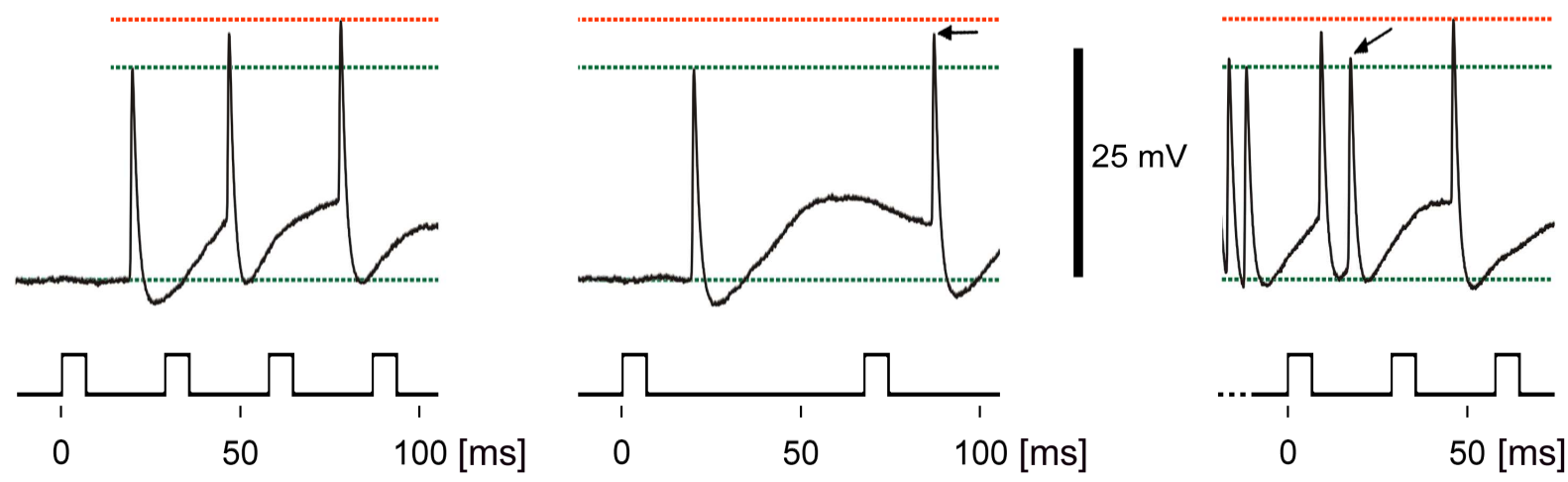

Figure 27: Three sections of one continuous recording of spikes and graded potentials to stimuli with different pause durations (pulse dur.: $7 \mathrm{~ms}$; pause dur.: $22 \mathrm{~ms}$ (left \& right) and $61 \mathrm{~ms}$ (middle); white noise; $70 \mathrm{~dB}$ SPL; see chapter 2.3.2 for stimulus paradigm). Green lines mark the resting potential and the amplitude of the first spike. Orange line indicates the maximal amplitude of a spike sitting on top of a graded potential. The arrows mark spikes of lower amplitudes in comparison to the maximum. 
Comparison to the behaviour of $A$. nigrovittata. In the natural duet of this species the male generates a calling song and the female replies with a single pulse after a constant latency. The pause between the male trigger pulse and the female answer - as perceived by the male - can vary and mainly depends on two factors. The first factor is the internal delay of the female which is approximately $25 \mathrm{~ms}$ (Dobler et al. 1994a). The second factor is the distance of the duetting partners. For example, a distance of $5 \mathrm{~m}$ would mean a travelling time for the sound waves of ca. $30 \mathrm{~ms}$ (see chapter 2.5 for calculations of the travelling time). Since TN1 responds best to the female answer and additionally shows a graded potential to the male carrier frequency (even at low intensities), the variable effect of pause duration between trigger (that causes a graded potential) and reply on the summation of an action potential and graded potential was tested (Figure 28A; for stimulus paradigm see chapter 2.3.2). Here, 3 examples are displayed, whereat only the amplitude difference of a spike (caused by the simultaneous occurrence of a graded potential) at different pause durations is compared to the amplitude of an action potential not sitting on a graded potential (which is taken as 100\%).

A

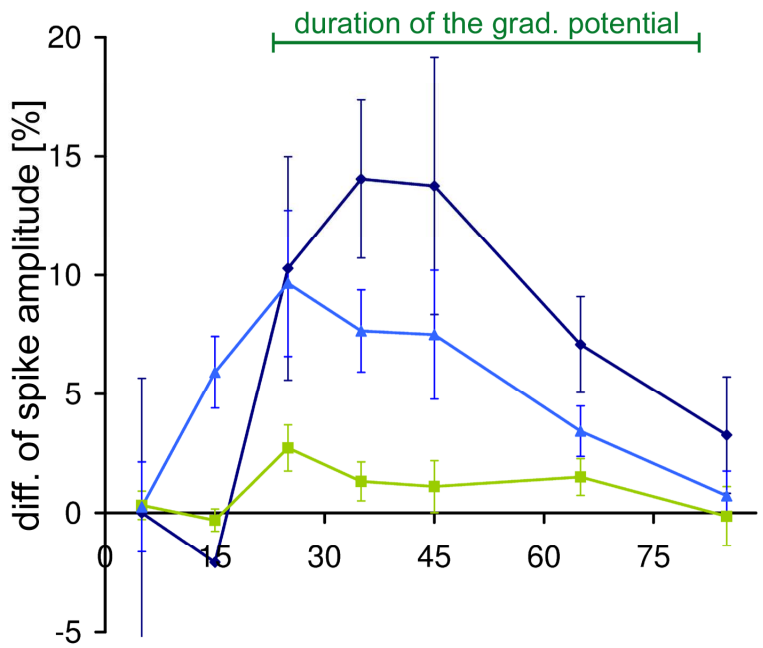

Pause duration [ms]
B mean spike amplitude difference

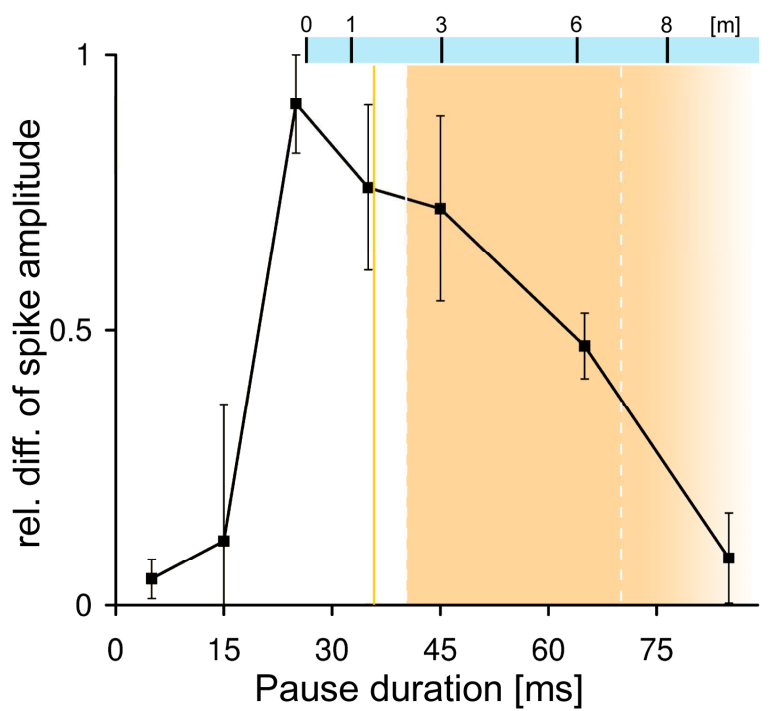

Figure 28: Difference of spike amplitude in TN1 between spikes sitting on top of a graded potential and spikes without simultaneous graded potential was measured to the female reply at different pause durations between male trigger pulse and female reply (see chapter 2.3.2 for stimulus parameters). A: Three individual responses where the difference of spike amplitude is displayed ( $n=5$; mean and SD). Green bar shows the mean latency and duration of the graded potential elicited by the male trigger only (data taken from 3 different individuals). B: Relative difference of spike amplitude of the responses shown in A (mean and SE). Orange box (until white dashed line) indicates preliminary data of positive male phonotaxis to an artificial female reply (obtained by Pierre Gras; higher pulse duration showed little or no response). Yellow line indicates the shortest latency of the female reply for which positive male phonotaxis was tested (Dobler et al. 1994b). For shorter pause durations no data is available but female replies can occur up to ca. $25 \mathrm{~ms}$ pause duration (Dobler et al. 1994a). Blue bar indicates the distance between the duetting partners (for calculation refer to chapter 2.5). 
The dependence of pause duration in each example has an optimum type character. For very short pauses (15 ms) also negative values were measured. This is due to the strongly pronounced repolarisation phase of the spike elicited by the male trigger (see Figure 27 right). If a spike occurs directly within this phase its amplitude can be smaller than a spike sitting on the resting potential. The latency and overall duration of the graded potential (elicited by the trigger pulse) can be seen in TN1 with stimulation of the male song only (performed in different individuals, other than those in which spike amplification was measured). For this, a necessary requirement is the lack of TN1's spike response to the male trigger syllable, since a spike conceals the beginning of the graded potential. The graded potential has a latency of $30.0 \pm 0.9 \mathrm{~ms}$ to the onset of the trigger syllable (Figure 28A, beginning of the green bar; $\mathrm{N}=3, \mathrm{n}=5$ for each individual; mean and SE; note that the $\mathrm{x}$-axis shows the pause duration and not the onset of the trigger syllable) to the trigger pulse. The graded potential's duration is $58.3 \pm 3.8 \mathrm{~ms}$ (green bar in Figure 28A; mean and SE) and therefore much longer than the stimulus $(7 \mathrm{~ms})$. This duration resembles the overall "time window" in which a summating effect might be observed. In other recordings of TN1 no difference in spike amplitude could be measured. This was partly due to the absence of spiking activity to $16 \mathrm{kHz}$ (in some individuals; therefore no comparison to a preceding spike without an increased amplitude was possible). The relative difference in spike amplitude (of the 3 examples shown in Figure 28A) is plotted in Figure 28B. A pronounced increase in amplitude can be seen between 25 and $65 \mathrm{~ms}$ pause duration. Preliminary data of the male behaviour to an artificial female reply with varying latency revealed a definite response to replies with pauses between 40 and $70 \mathrm{~ms}$ (orange box until white dashed line in Figure 28B preliminary data obtained by Pierre Gras). Longer pauses showed few or no responses (fading orange box on the right side). The yellow line in Figure 28B indicates the shortest latency of an artificial female reply that was tested and elicited a positive male phonotaxis (see Dobler et al. 1994b). Shorter pause durations were not measured, but can be expected to elicit a male response up to the naturally occurring shortest pause of ca. 25 ms (Dobler et al. 1994a). 


\subsubsection{Ascending neuron $x(\mathrm{ANx})$}

During the search for auditory neurons in the lateral protocerebrum in the brain a recording of a yet unknown ascending interneuron was obtained. The physiological and morphological characteristics clearly differ from the other neurons already known from thoracic recordings. Since morphology is only known for the brain, it is unknown whether its soma is also located in the thorax as for most of the other ascending neurons. Therefore, in the following this ascending neuron is only indexed with an " $x$ ". In addition to a clear physiological response to acoustic stimuli, ANx also seems to be slightly excited by vibratory stimulation.

Morphology. An important criterion for discrimination from the known ascending neurons was ANx morphology (Figure 29). The thick axon runs in the dorsal medial portion of the circumoesophageal connective through the trito- and deutocerebrum without branching. Then, the axon extents to the lateral protocerebrum and arborises ventrally. The arborisations show beaded endings, which is an indicator for presynaptic structures and therefore the output region of ANx. The overall extent of the arborisations is restricted to the caudal protocerebrum that strongly reminds of the morphology of vibratory neurons (own observation; also see chapter 3.1.7).
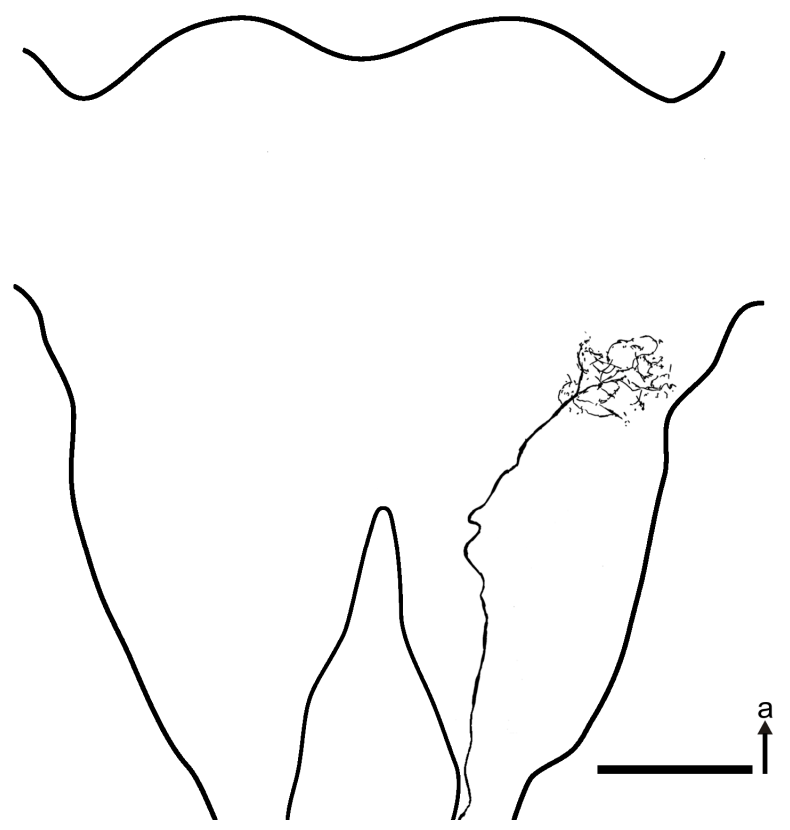

Figure 29: Drawing of ANx from a Cy3staining done in the brain. Bar represents $250 \mu \mathrm{m}$. a=anterior.

Intensity and frequency dependence of $A N x$ 's response. Stimulation with $50 \mathrm{~ms}$ pulses and $28 \mathrm{kHz}$ carrier frequency reveals the strongest response at $40 \mathrm{~dB} \mathrm{SPL}$ (Figure 30A). The responses to ultrasonic frequencies show lower spiking thresholds 
than responses to 16 or $8 \mathrm{kHz}$. The overall spiking activity is very variable, which can be seen at the large standard deviations for all frequencies. The difference in response to sonic and ultrasonic frequencies is more clearly pronounced when presenting the male song pattern at increasing intensities (Figure 30B; see chapter 2.3.2 for stimulus paradigm). Again, a high spiking activity is found for $28 \mathrm{kHz}$ at $40 \mathrm{~dB}$ SPL. An additional response peak is found at $70 \mathrm{~dB}$ SPL (at this intensity also the response to $38 \mathrm{kHz}$ reaches its maximum). ANx's spiking activity is insensitive to sonic frequencies with a threshold of approximately $70 \mathrm{~dB}$ SPL. The response at $8 \mathrm{kHz} 90 \mathrm{~dB}$ SPL was very variable. The spiking activity to the female's reply in an artificial duet is increased in the complete ultrasonic frequency range tested (Figure 30C). Also the stimulation with $12 \mathrm{kHz}$ elicits responses, whereby 16 and $20 \mathrm{kHz}$ were responded to the least.

A

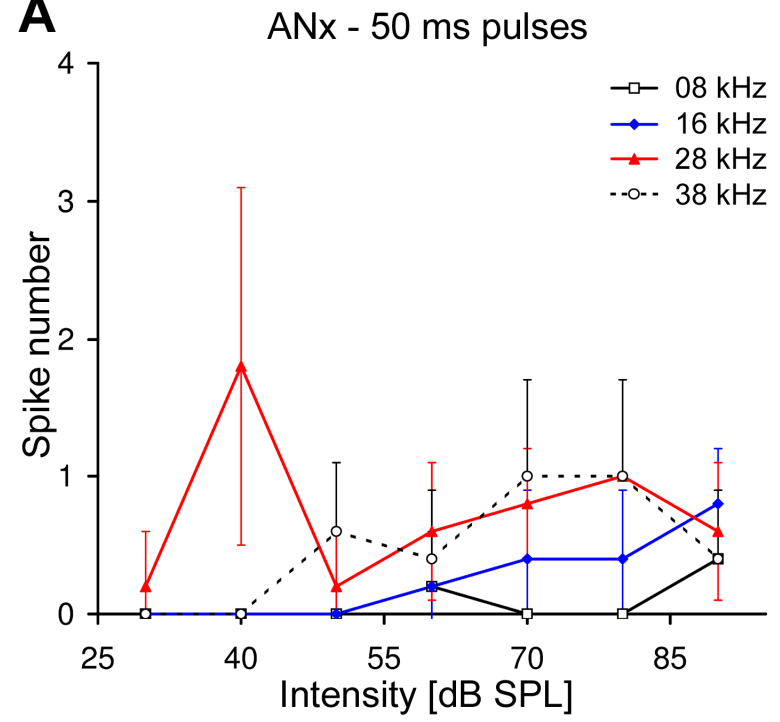

C frequency of the $Q$-reply

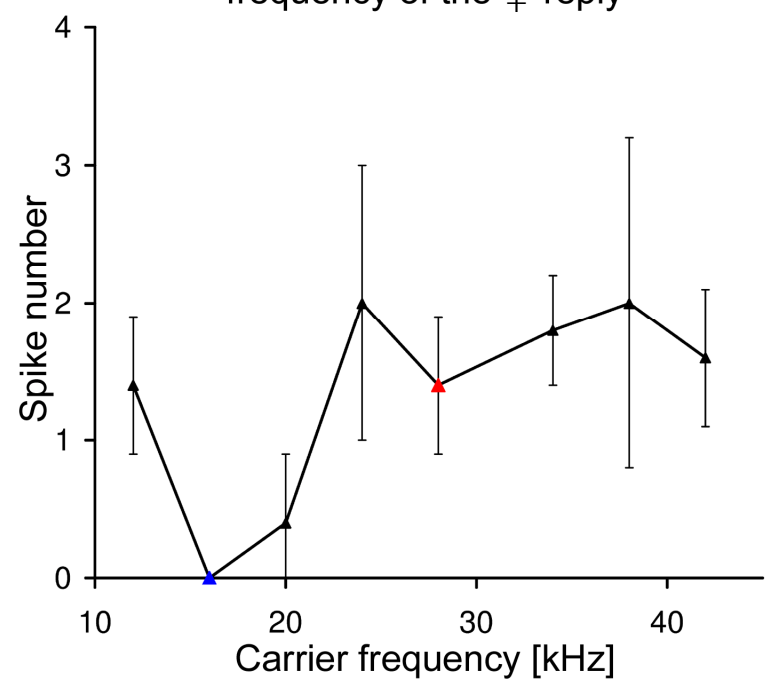

B
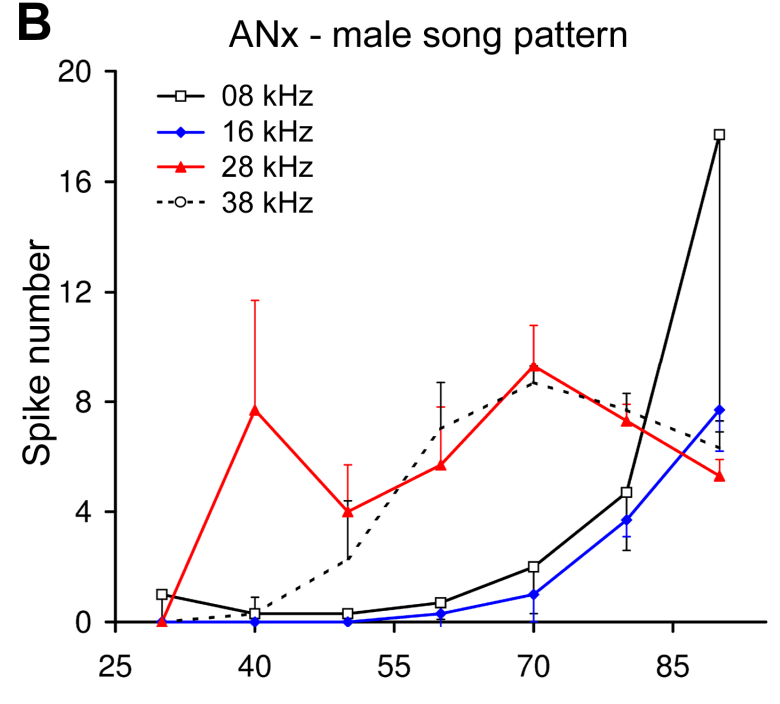

Figure 30: Response of ANx to different acoustic stimuli recorded in the brain $(n=5$; means and $S D)$. Blue colouration - carrier frequency of the male song $(16 \mathrm{kHz})$ and red colouration - carrier of the female reply $(28 \mathrm{kHz})$. A: Response of $\mathrm{ANx}$ to standard stimuli (see chapter 2.3.2). B: Intensity dependent response of $A N x$ to the male song pattern at 8. 16, 28 and $38 \mathrm{kHz}$ (see chapter 2.3.2 for stimulus paradigm). C: Mean response to the female answer with varying carrier frequency in an artificial duet (see chapter 2.3.2 for stimulus paradigm). 
Response of $A N x$ to varying temporal parameters. ANx's response shows a strong dependence for pause duration with optimum type characteristics (Figure 31). The highest spike activity is found at $22 \mathrm{~ms}$ pause duration, whereby nearly no response can be observed to the shortest pause duration presented. This dependence of $\mathrm{ANx}$ relative spike numbers to different pause durations closely correlates with the female behaviour. On the other hand, the high threshold for $16 \mathrm{kHz}$ (see Figure 30B) seems to exclude ANx to be involved in the possessing of the temporal parameters important for male song recognition.
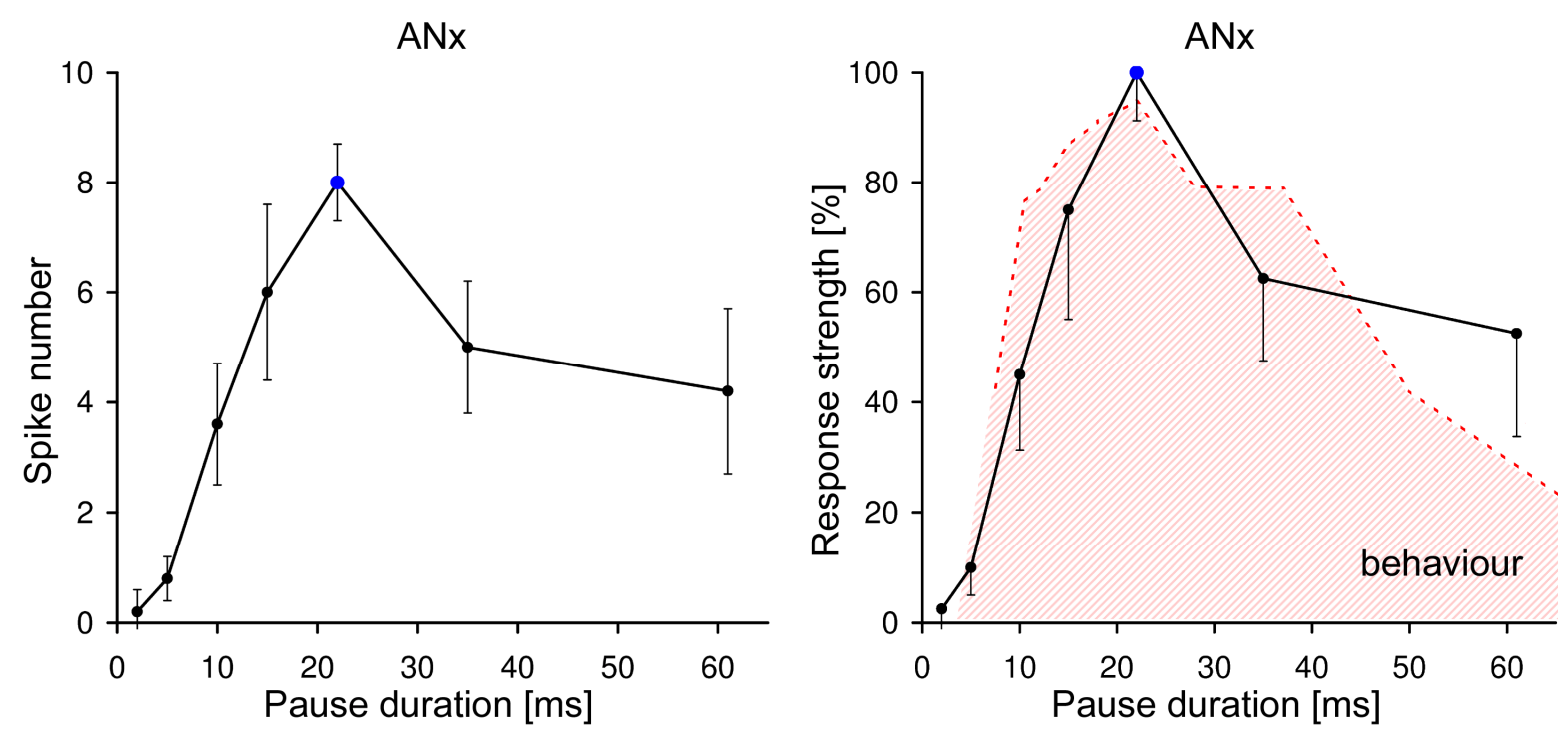

Figure 31: Spike number (left) and magnitude of response (right) to different pause durations (white noise; $70 \mathrm{~dB}$ SPL; $\mathrm{n}=5$; see chapter 2.3.2 for stimulus paradigm; means and SD). Blue data point indicates the male song pause duration. Red shaded area indicates the female behaviour $(\mathrm{N}=5$, modified from Dobler et al. 1994a).

The response to varying pulse durations of $A N x$ is shown in Figure 32. Short pulse durations, as found for the female answer or for bat calls, evoke the highest spiking activity. Pulse durations longer than $10 \mathrm{~ms}$, contrary, evoke nearly no response. 


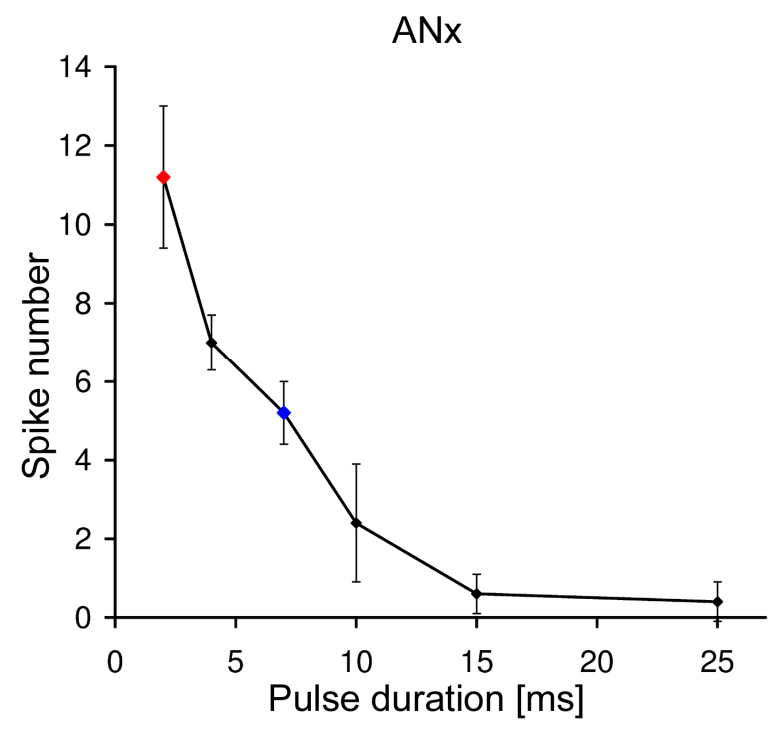

Figure 32: Spike number of $A N x$ to different pulse durations (white noise; $70 \mathrm{~dB}$ SPL; $\mathrm{n}=5$; see chapter 2.3.2 for stimulus paradigm; mean and SD). Blue: male song pulse duration, Red: female answer pulse duration.

Spike latencies in $A N x$. The shortest spike latencies for ANx are $24.9 \pm 0.6 \mathrm{~ms}$ $(\mathrm{n}=5$; mean and SD) and are found for $28 \mathrm{kHz}$ at $80 \mathrm{~dB}$ SPL (50 ms pulse duration). This, compared to the other ascending neurons, is very long. The response of $\mathrm{ANx}$ is strongest to short pulse durations but the latencies - taken from the best response to the stimulus paradigm shown in Figure 32 - are relatively high with $28.5 \pm 0.7 \mathrm{~ms}$ $(\mathrm{n}=5$; mean and SD).

\subsubsection{Ascending vibratory neurons}

Besides intracellular recordings of neurons that respond to acoustic stimuli, also neurons are frequently found that respond best to the vibrational test (see chapter 2.3.2). Characteristically, these neurons also respond to low carrier frequencies at high intensities. In the following two examples (out of 4 ) of vibratory neurons will be presented.

Morphology. In Figure 33 the morphologies of two ascending vibratory neurons are given. Both neurons were stained in the same individual. The axon mostly enters the brain in dorsal medial portions and extends to the protocerebrum, where it runs directly to the caudal lateral protocerebrum (right neuron). This change of direction often results in a typical bend (marked by the arrow in Figure 33 left). This bend is also found in the vibratory neuron on the left side although its axon enters the brain in more lateral portions (rarely observed). The major arborisations (expanding ventrally) 
are restricted to the caudal protocerebrum and either have a bushy appearance with no prominent collaterals (right neuron) or have a few collaterals with very sparse branching (left neuron). The beaded endings at the axonal branches are characteristically pronounced and clearly visible (Figure 33 right).
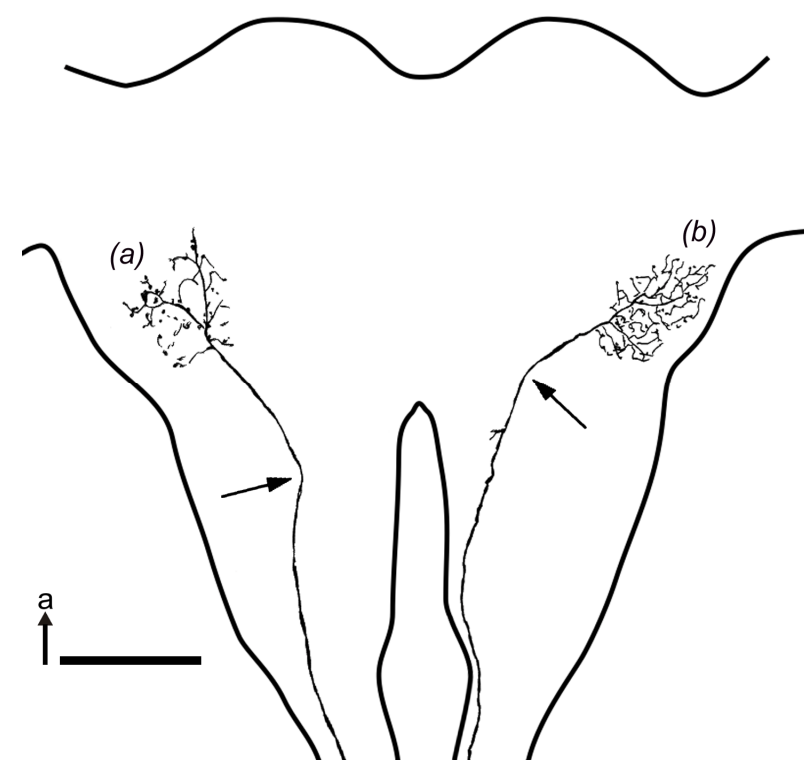
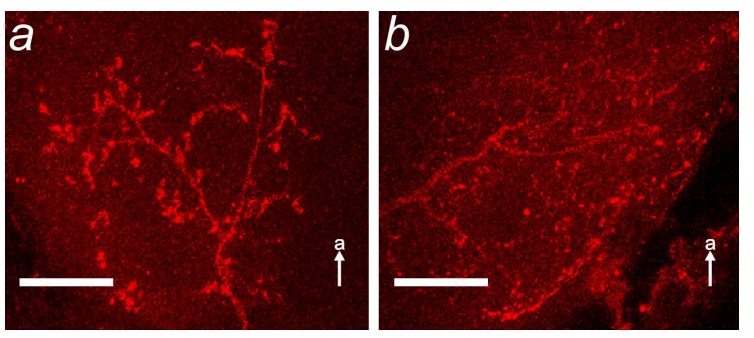

Figure 33: Left: Drawing from a Cy3-staining of two vibratory neurons in each hemiganglion of the same brain of one individual. Bar represents $200 \mu \mathrm{m}$. Right: Detailed view of the major arborisation area of both neurons presented in the drawing (left (a) and right (b)). Bar represents $50 \mu \mathrm{m}$. Note the clearly beaded structures. a=anterior.

Intensity dependence of vibratory neuron's response at various frequencies. Besides spiking activity to vibration also responses to low frequent air borne sound at high intensities can be observed.
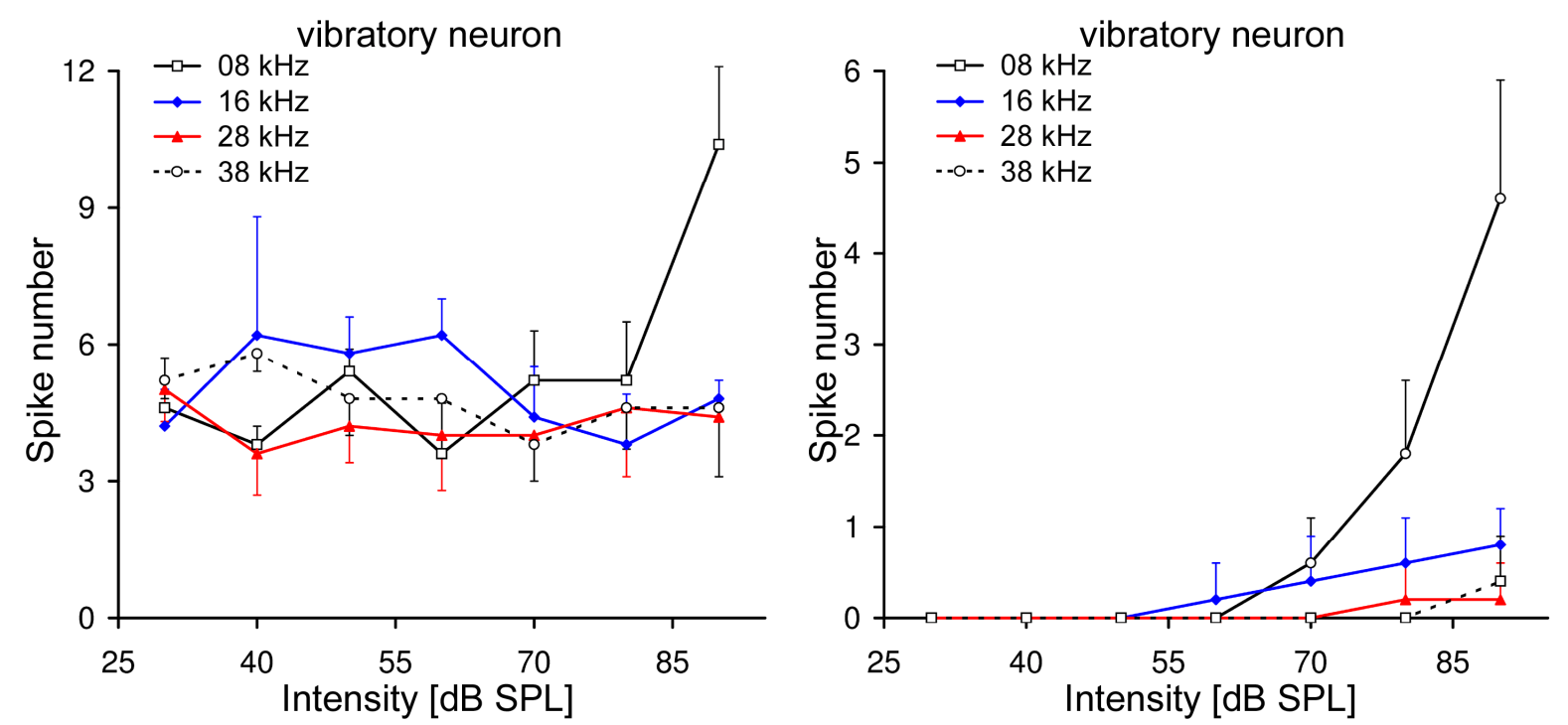

Figure 34: Response of vibratory neurons to standard stimuli (see chapter 2.3.2) ( $n=5$ for each graph; means and SD). Left graph corresponds to the left neuron in Figure 33 and vice versa for the right graph. Blue colouration - carrier frequency of the male song $(16 \mathrm{kHz})$ and red colouration - carrier of the female reply $(28 \mathrm{kHz})$. 
In Figure 34 the intensity dependent responses for both neurons are displayed. The left vibratory neuron (left graphs come from the left neuron in Figure 33 and vice versa) shows a high spontaneous activity. The only clear response to acoustic stimulation is seen at $8 \mathrm{kHz} 90 \mathrm{~dB}$ SPL. Contrary, the right neuron reveals no spontaneous activity. The threshold for the response to $8 \mathrm{kHz}$ is $20 \mathrm{~dB}$ lower compared to the left neuron. To a smaller extent, than seen for $8 \mathrm{kHz}$, also a response to $16 \mathrm{kHz}$ and at very high intensities also slightly to ultrasound can be observed.

Spike latencies in vibratory neurons. The shortest latency for the neuron with the high spontaneous activity could not be obtained since all measurements had similar values (even for $8 \mathrm{kHz}$ at $90 \mathrm{~dB} \mathrm{SPL}$ ). The neuron with no spontaneous spiking activity had shortest latencies of $21.2 \pm 3.4 \mathrm{~ms}(n=5$; mean and SD) which is similar to the latencies of sound activated ascending neurons. 


\subsection{Brain neurons}

\subsubsection{Local brain neuron 1 (LBN1)}

In addition to intracellular recordings of ascending neurons in the brain, the major aim of this study was to identify local brain neurons that may be involved in the auditory processing of the conspecific song. LBN1 is such a candidate that is possibly critically involved in male song recognition. It was rarely recorded and a recording mostly lasted a few minutes only.

Morphology. One successful complete staining with a fluorescent tracer shows the morphology of LBN1 (Figure 35A-C). The overall spatial extent is restricted to the auditory neuropil in the lateral protocerebrum, where its soma is located in the medial dorsal region of the hemiganglion. The primary neurite runs in a ventral lateral direction and gives off minor branches that take course to a posterior dorsal region. At this point the primary neurite thickens and two major collaterals have their origin, running anterior ventral, and also one major axon, running in a medial anterior and subsequently in a lateral ventral direction.

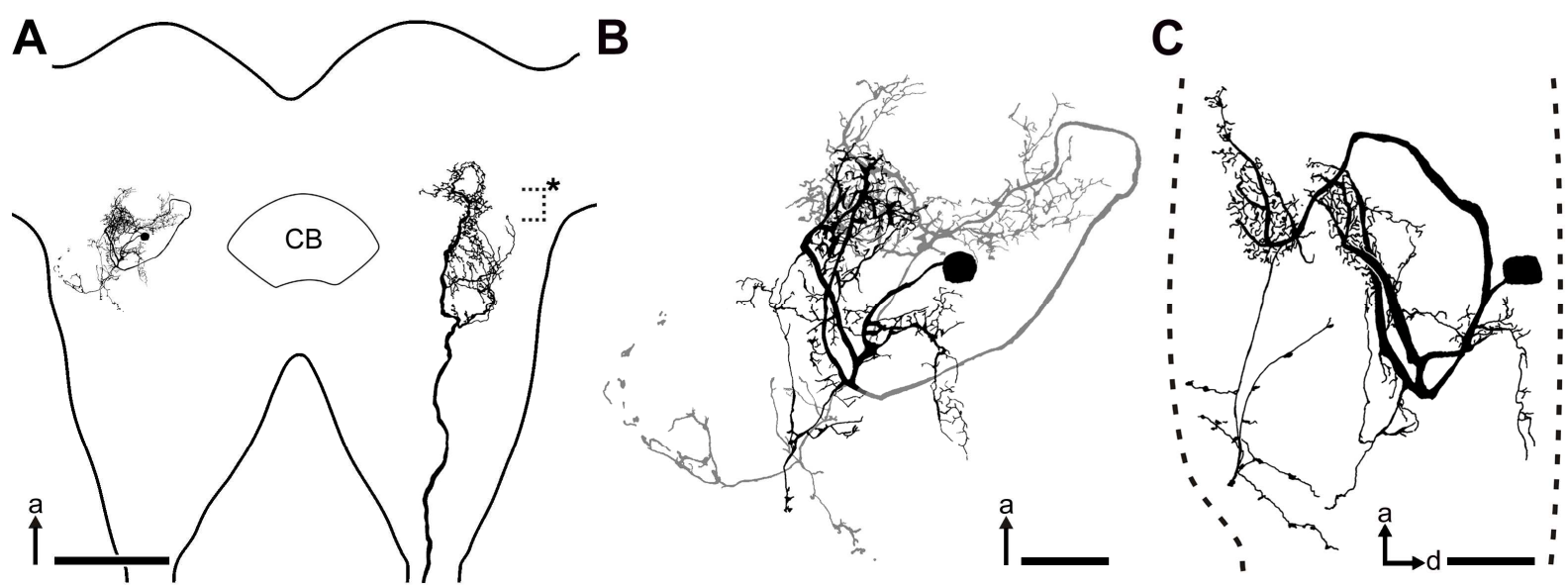

Figure 35: Morphology of LBN1 and AN1 in the brain. A: Drawings of LBN1 and AN1 from Cy3stainings in different individuals (staining of AN1 was performed by A. Stumpner). The asterisk marks the area of possible connection between AN1 and LBN1. Bar represents $250 \mu \mathrm{m}$. CB=central body; $\mathrm{a}=$ anterior. B: Detailed view of the LBN1 arborisations. The dendritic branches are shown in black, while axonic arborisations are displayed in grey. Bar represents $50 \mu \mathrm{m}$. $\mathrm{a}=$ anterior. C: Lateral view on LBN1 (rotated by about $65^{\circ}$ around the vertical axis when compared to B). Note the clear separation of the dendritic (right) and axonal (left) branches. The dashed lines indicate the borderlines of the brain. Data from 3D-reconstruction of a confocal stack using AMIRA 5.2.0 (Visual imaging); the lower resolution for the z-axis leads to apparently thicker branches. Bar represents $50 \mu \mathrm{m}$. $a=a n t e r i o r$; $\mathrm{d}=$ dorsal. 
The arborisations of the two major collaterals have fine endings indicating a postsynaptic function, while the arborisations of the axon have many beaded endings that are typical for presynaptic structures. The densest axonic branching pattern reminds of a "U" shape whereby each "side" extends anteriorly (grey in Figure 35B). It is found more ventrally than the densest dendritic arborisation (Figure 35C). A minor single branch of the axon takes course to the posterior end of the lateral protocerebrum and branches off medially and laterally. Also a minor dendritic branch extends to more posterior portions. There seems to be no physical connection between the two arborisation areas, since they appear to be situated in different layers of the protocerebrum. The branching pattern of AN1 from a different individual is also displayed in Figure 35A (LBN1 seems to be directly connected to AN1 - see below). For alignment of the two neurons the position of the central body was taken as a reference. The possible region of connection of AN1 to LBN1 is marked by an asterisk, where AN1's major axon runs ventrally to medial portions of the protocerebrum while giving off dense arborisations (see Stumpner 1997).

Intensity dependence of $L B N 1$ 's responses at various frequencies. If one compares the responses of LBN1 and AN1 to standard stimuli (Figure 36A, B), striking similarities between the two neurons are obvious: both neurons show optimum type curves with maximum response strength at sonic frequencies at exactly the same intensities. Among 8, 16, 28 and $38 \mathrm{kHz}$ both neurons respond strongest at $16 \mathrm{kHz}$ and much less at 28 and $38 \mathrm{kHz}$. However, LBN1 is significantly less sensitive by $7.8 \mathrm{~dB}$ at $16 \mathrm{kHz}$ than $\mathrm{AN} 1$ (response threshold for $\mathrm{AN1}$ : $32.7 \pm 1.4 \mathrm{~dB}$ SPL, $N=11$; response threshold for LBN1: $40.5 \pm 1.8 \mathrm{~dB}$ SPL, $N=6$; mean and SE; $p=0.018$, 2-tailed Mann-Whitney U-Test; see also Figure 44B). At ultrasonic frequencies LBN1 rarely responds up to intensities of $70 \mathrm{~dB}$ SPL. Moreover, LBN1 shows an overall much weaker response than AN1 (maximum spike numbers at $16 \mathrm{kHz} 2.5 \pm 0.6$ in 6 LBN1; $8.9 \pm 0.6$ in 13 AN1 recorded in the brain; means and SE). Recordings of LBN1 to models of male calling songs (at different frequencies and $60 \mathrm{~dB} S P L$ ) reveal a reliable response at the natural male calling frequency only (Figure 36C left). In comparison, spiking of AN1 recorded in the brain occurs also at other carrier frequencies, though response strength is low (Figure 36C right). 
A

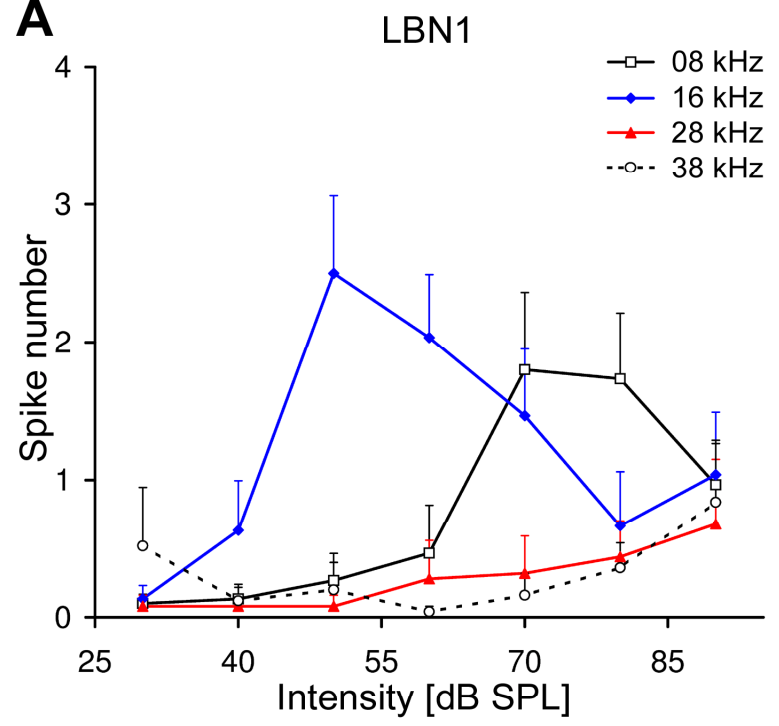

C

$25 \mathrm{mV}$

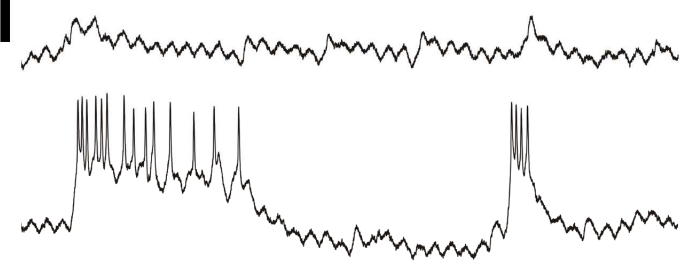

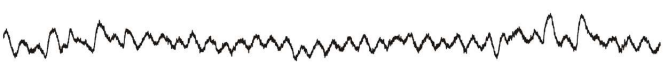

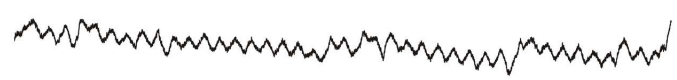

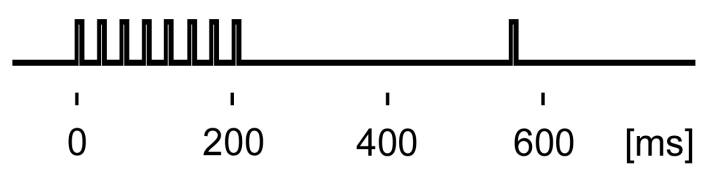

B

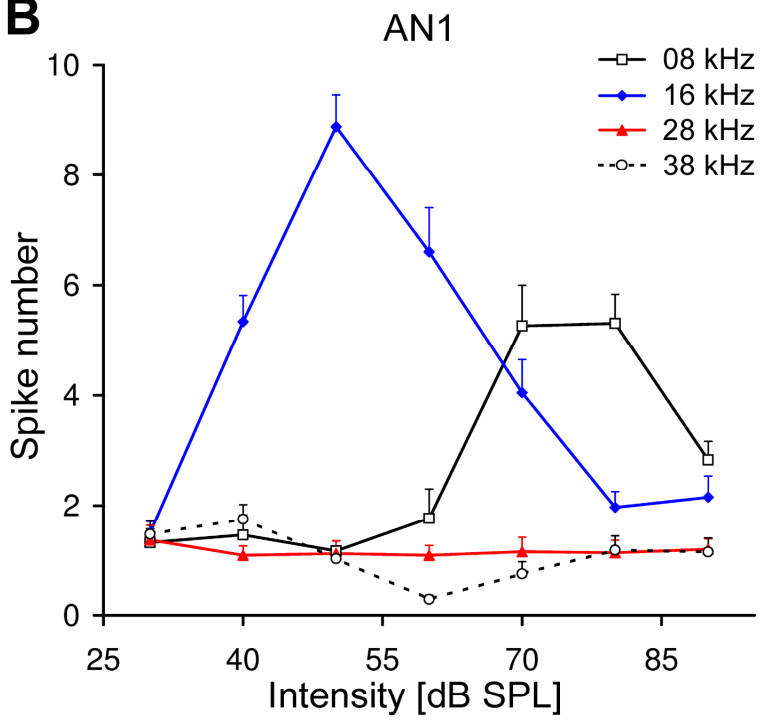

$08 \mathrm{kHz}$

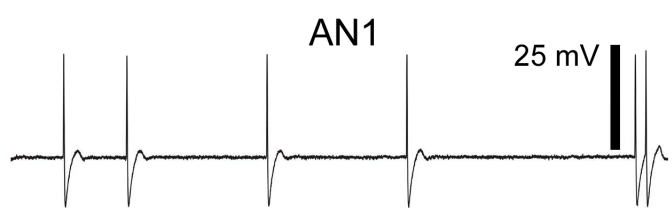

$16 \mathrm{kHz}$

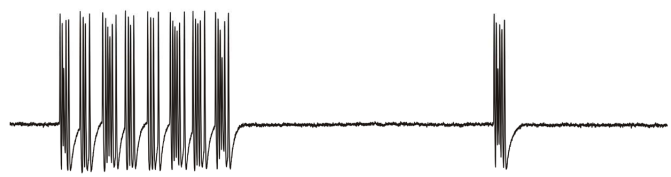

$28 \mathrm{kHz}$

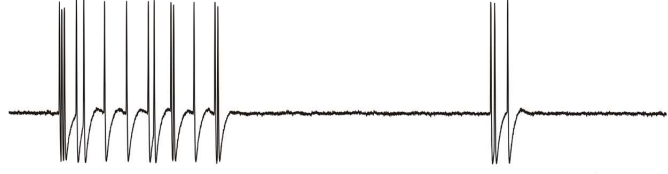

$38 \mathrm{kHz}$
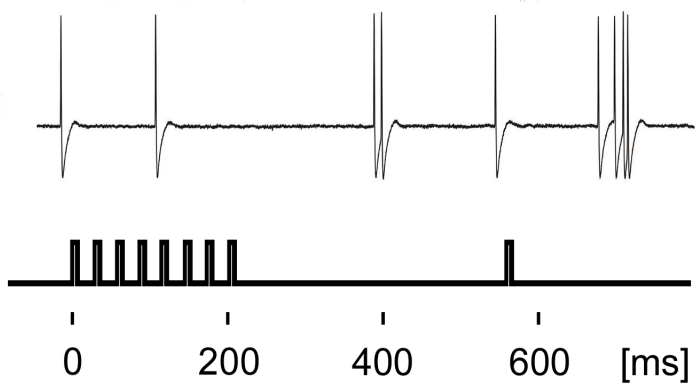

Figure 36: Responses to the standard stimuli. Blue colouration indicates the carrier frequency of the male song $(16 \mathrm{kHz})$ and red colouration the carrier of the female reply $(28 \mathrm{kHz})$. A: Intensity dependent responses of LBN1 (N=5-6; means and SE). B: Intensity dependent response of AN1. Note the different scale of the $y$-axis in comparison to $A(N=13$; means and $S E)$. $C$ : Example recordings of LBN1 left and AN1 right to the male calling song at $60 \mathrm{~dB}$ SPL (for stimulus paradigm see chapter 2.3.2). The regular fluctuations seen in LBN1-traces are $50 \mathrm{~Hz}$-hum.

Comparison of $L B N 1$ and $A N 1$ latencies. From the data presented so far it is well conceivable that LBN1 is directly postsynaptic to AN1. This hypothesis appears to be confounded by a marked spike latency difference between action potentials of AN1 (19.1 $\pm 0.5 \mathrm{~ms}$ in the brain at $16 \mathrm{kHz} 50 \mathrm{~dB}$ SPL; $\mathrm{N}=13$; mean and SE) and of LBN1 (29.9 $\pm 1.1 \mathrm{~ms}$; $\mathrm{N}=6$; mean and SE). However, recordings of LBN1 neurons 
show prominent singular excitatory postsynaptic potentials (EPSPs) summing up to eventually evoke an action potential (Figure 37). This gives a remarkably large difference of about $9 \mathrm{~ms}$ between latency of the first EPSP $(20.8 \pm 0.5 \mathrm{~ms}$ at $16 \mathrm{kHz}$ $50 \mathrm{~dB}$ SPL; $\mathrm{N}=6$; mean and SE) and latency of the first action potential in LBN1. The difference between the mean latencies of initial LBN1-EPSPs and mean latencies of AN1-spikes in the brain is in the order of $2 \mathrm{~ms}$ (Figure 38 ).

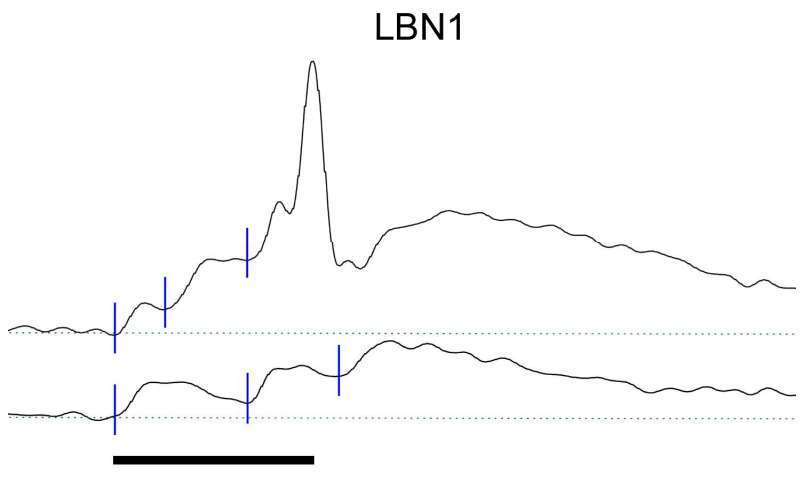

Figure 37: Graded potentials in LBN1. Both example recording show prominent singular EPSPs. The beginning of each EPSP is marked by a blue line. The resting membrane potential is indicated by a green dotted line. Upper trace: Summation of EPSPs until the spiking threshold is reached. Lower trace: The interval between the EPSPs is too large to summate and to reach the spiking threshold. Bar represents $15 \mathrm{~ms}$.

This leads to the conclusion that a latency of $30 \mathrm{~ms}$ or more of a second order interneuron can be due to a latency of the first order interneuron (AN1) in the thorax of around $15 \mathrm{~ms}$, a propagation time between thorax and brain in the axon of AN1 of around $5 \mathrm{~ms}$ and a synaptic delay from AN1 to LBN1 of $2 \mathrm{~ms}$ plus a long time between the initial EPSP and the first action potential in LBN1 of around $10 \mathrm{~ms}$ (Figure 38).

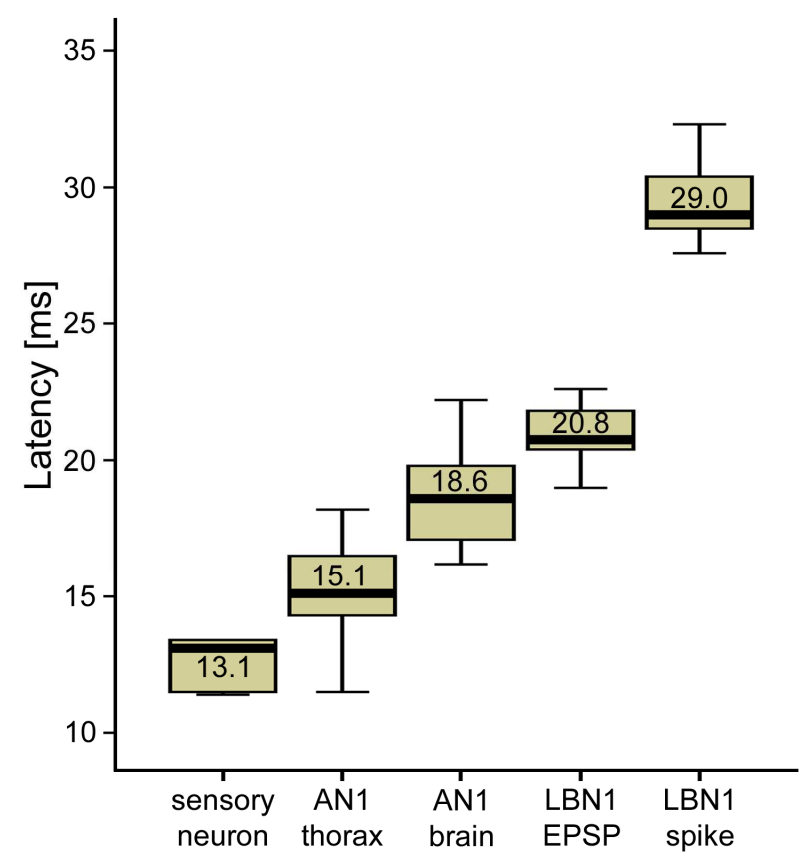

Figure 38: Box plot (see chapter 2.5) of the latencies of sensory neurons (best tuned to $16 \mathrm{kHz} ; \mathrm{N}=5)$, AN1 thorax $(\mathrm{N}=23$ - from $\mathrm{A}$. Stumpner), AN1 brain $(\mathrm{N}=13)$, LBN1 EPSP $(\mathrm{N}=6)$ and LBN1 spikes $(\mathrm{N}=6)$ at $16 \mathrm{kHz} 60 \mathrm{~dB}$ SPL (50 ms stimulus; values of medians are given in the figure). 
Comparison of LBN1 and AN1 spike/EPSP frequencies. Is there additional evidence that LBN1-EPSPs are directly evoked by AN1 action potentials? For this, the frequency of singular EPSPs of LBN1 was measured that obviously are evoked by single action potentials of the presynaptic neuron (marked beginnings of single EPSPs in Figure 37). The maximum EPSP-frequency occurring among the first EPSPs of LBN1 (prior to spiking) was then compared to the maximum action potential-frequency of AN1 at various intensities of $16 \mathrm{kHz}$, the frequency of preferred response (Figure 39 left). This maximum spike frequency always occurred during the first burst of action potentials. The average EPSP frequency and spike frequency of the two neurons are very similar. The only larger average difference is seen at $40 \mathrm{~dB}$ SPL. However, even at that intensity the data of single spike/EPSP frequencies of AN1 and LBN1 overlap (Figure 39 right). This finding further supports a direct connection between AN1 and LBN1.
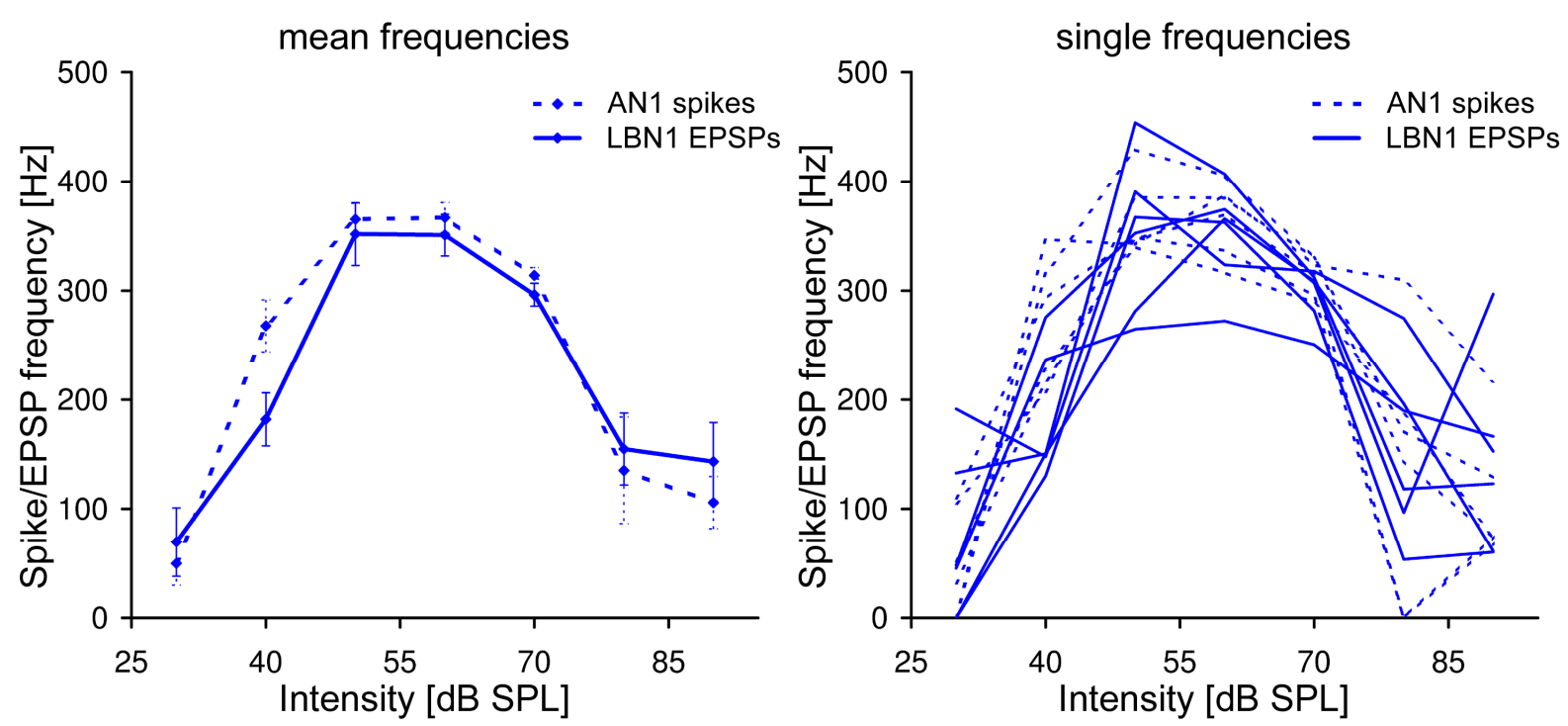

Figure 39: Intensity dependence of AN1-spikes and LBN1-EPSPs at $16 \mathrm{kHz}$ (AN1, N=6; LBN1, N=6). Left: Mean spike/EPSP frequencies (means and SE). Right: Comparison of individual spike/EPSP frequencies.

A comparison of independently recorded AN1 (brain) and LBN1 (Figure 40) at identical carrier frequencies and intensities indicates a clear covariance $\left(R^{2}=0.758\right)$ between maximum spike frequency (average of 6 AN1) and spike number in 6 LBN1 (Spearman rank-order correlation coefficient, $\mathrm{df}=26, \mathrm{p}<0.001$ ). If spike frequency of AN1 is above ca. $270 \mathrm{~Hz}$, spike number in LBN1 gets higher than 1. At lower AN1spike frequencies LBN1 produces - with one exception - less than one spike on average. 


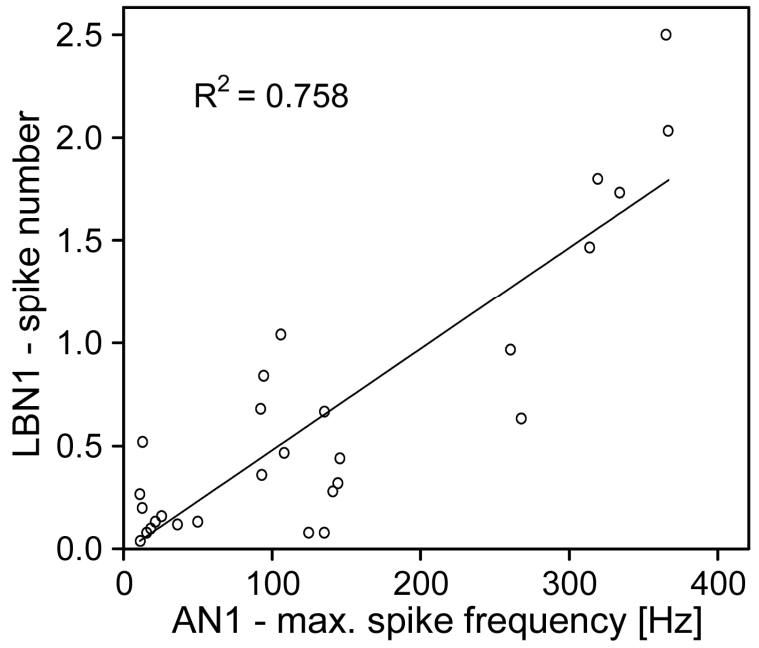

Figure 40: Correlation between average maximum spike frequency of AN1 and spike number of LBN1 to standard stimuli (see chapter 2.3.2). Each point represents the mean of 6 AN1 and 5-6 LBN1 recorded in different individuals. If no or only one spike occurred during the window of evaluation (150 ms), the spike frequency was taken as zero. The line shows the linear regression; $R^{2}$ gives the square of the correlation coefficient.

Carrier frequency dependent IPSPS in LBN1. In recordings of LBN1 also inhibitory postsynaptic potentials (IPSPs) have been observed in addition to the EPSPs. In the majority of cases, however, the visible effect of inhibition seems to be a lowering of the EPSP amplitude by an overlapping IPSP. Thus, a differentiation between EPSP and IPSP is not possible. During depolarisation though, IPSPs can be more clearly identified (Figure $41 \& 42$ ). At high stimulus intensity two subsequent IPSPs can be observed in the averaged recordings displayed in Figure 41 (average contains 5 repetitions of the same stimulus). The potential reason for this biphasic nature of the IPSP will be discussed later.

Hidden IPSPs in LBN1

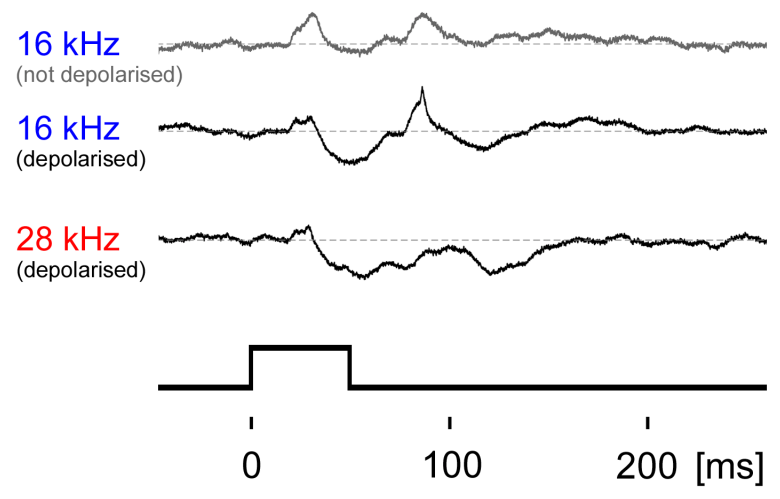

Figure 41: Recording examples of LBN1 without and with current induction (ca. $+0.5 \mathrm{nA}$ ) at 16 and $28 \mathrm{kHz}$ at $90 \mathrm{~dB} \mathrm{SPL}$ (averages of 5 repetitions; 50 ms stimuli).

A depolarisation during the intracellular recording of LBN1 allows the approximate detection of the IPSP-thresholds at various frequencies. In Figure 42 example recordings of LBN1 are displayed at 60 and $90 \mathrm{~dB}$ SPL for 8, 16, 28 and $38 \mathrm{kHz}$. At low intensities strongest IPSPs are observed at $28 \mathrm{kHz}$ thereby revealing a clear dependence on carrier frequency. At the highest intensity tested, also the 
responses to lower frequencies demonstrate the occurrence of an IPSP. The lowest thresholds for detectable IPSPs are found at frequencies between 28 and $34 \mathrm{kHz}$ (ca. $50 \mathrm{~dB}$ SPL). Small amplitude IPSPs occurring at lower intensities, however, may still be hidden in simultaneous EPSPs even at a depolarised state.
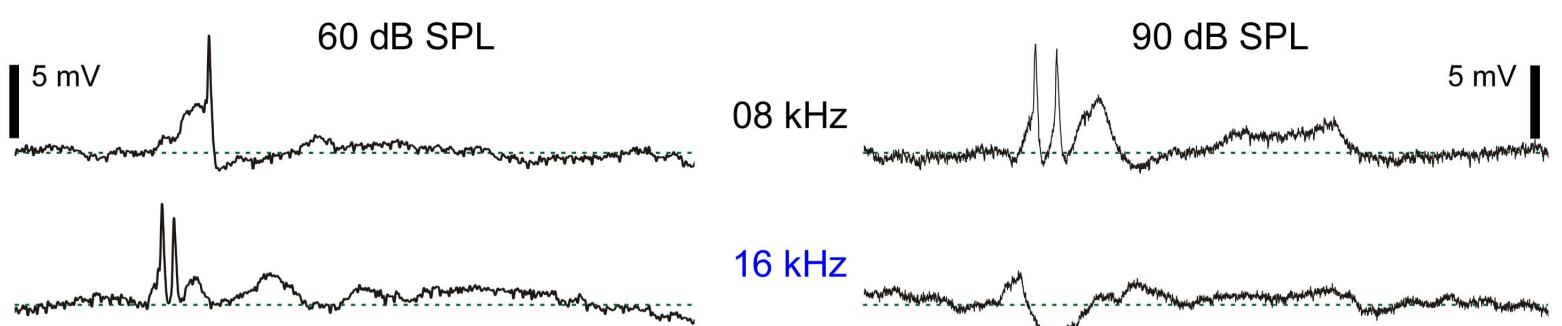

$16 \mathrm{kHz}$
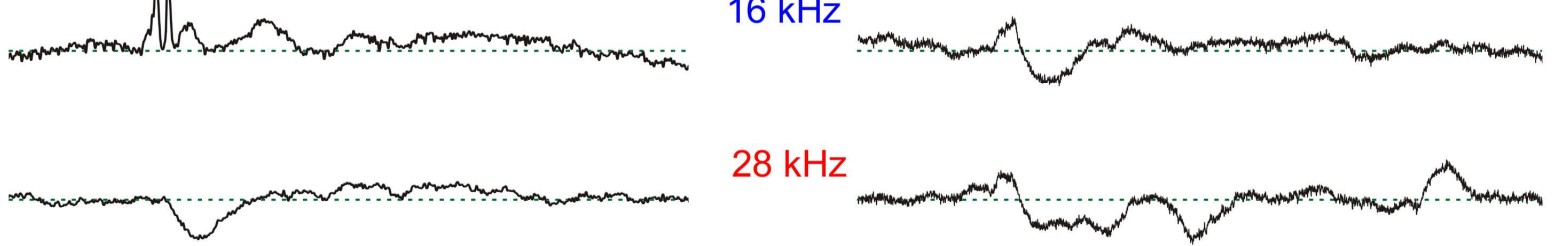

$28 \mathrm{kHz}$
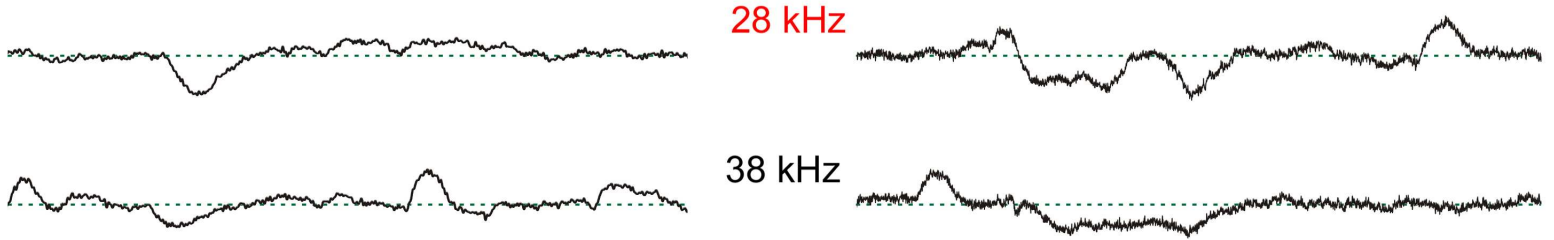

$38 \mathrm{kHz}$

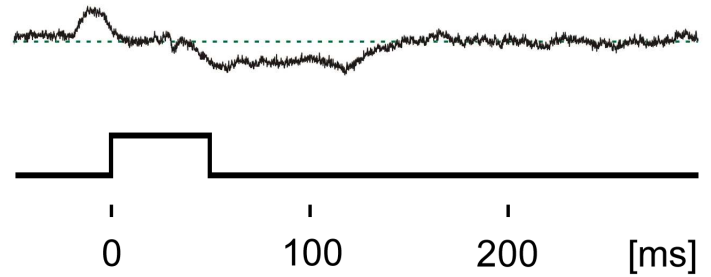

Figure 42: Example recordings of a depolarised LBN1 (ca. $+0.5 \mathrm{nA}$ ) for $8,16,28$ and $38 \mathrm{kHz}$ at 60 (left) and $90 \mathrm{~dB}$ SPL (right) (50 ms stimulus).

If one compares latencies of EPSPS and IPSPs, the distribution of all IPSPlatencies measured is clearly shifted to longer values as compared to the majority of EPSP-latencies (Figure 43). The EPSPs have a mean latency of $22.4 \pm 0.8 \mathrm{~ms}(\mathrm{~N}=4$, $n=204$; mean and SE) whereas the IPSP-latencies are $32.8 \pm 0.5 \mathrm{~ms}(\mathrm{~N}=4, \mathrm{n}=107$; mean and SE) (means include latencies of various frequencies and intensities at the same temporal parameters). A latency difference of $12.9 \pm 0.8 \mathrm{~ms}$ (measured when EPSP and IPSP occurred in combination during standard stimuli; $N=4, n=47$; mean and SE) suggests at least one, possibly several additional interneuron(s) to be involved in the inhibitory pathway to LBN1 as compared to the excitatory pathway. In Figure 43 a secondary peak of EPSP-occurrences is found (at 27 to 28 ms latency; marked by an asterisk). All EPSPs contributing to this peak arise from frequencies where AN1 responded weak due to inhibitory influence. The first peak in the histogram with the shortest latencies, therefore, mainly comes from frequencies where AN1 responded best ( 50 and $60 \mathrm{~dB} \mathrm{SPL}, 16 \mathrm{kHz}$ ). The earliest beginnings of IPSPs stem from ultrasonic frequencies and closely overlap the beginning of spiking in LBN1 at $16 \mathrm{kHz}$ (50 dB SPL; arrow in Figure 43). One should have in mind, 
though, that spiking in AN1 at ultrasonic frequencies also had longer latencies than at $16 \mathrm{kHz}$.

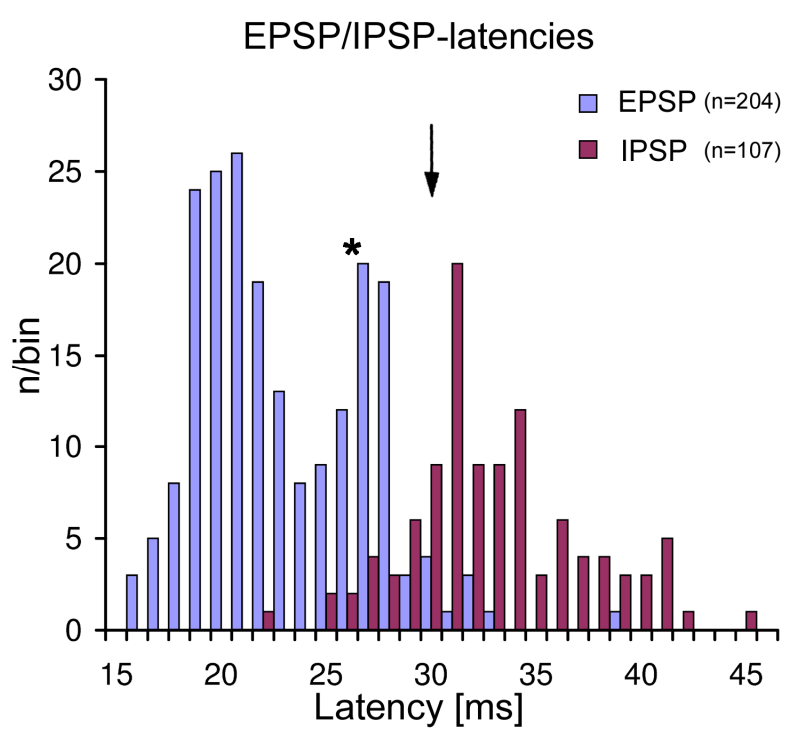

Figure 43: Histogram of EPSP- and IPSPlatencies in LBN1. Asterisk marks a secondary peak of EPSP occurrence. The arrow indicates the beginning of spiking in LBN1 at $16 \mathrm{kHz}$ $50 \mathrm{~dB}$ SPL)

Comparison of LBN1's response to the female behaviour. Summation of EPSPs in LBN1 obviously depends on spike frequency in AN1 with a minimal frequency for summation being around $40 \mathrm{~Hz}$ (ca. $25 \mathrm{~ms}$ singular EPSP-duration; $\mathrm{n}=44$ ). This summation remains subthreshold below a certain spike frequency of AN1 (and of course also depends on stimulus duration). As a necessary consequence, LBN1 is not brought to spiking with stimuli evoking only singular or widely spaced action potentials in AN1. This explains the higher threshold of LBN1 as compared to AN1. Therefore, a "spike frequency filter" during processing of the input into LBN1 has the consequence of strengthening the intensity dependence at the preferred frequencies resulting in a closer correlation to the female behaviour (Figure 44A). In addition, this effect of spike frequency filtering is supported by a delayed synaptic inhibition, obviously further reducing probability of spiking especially in the ultrasonic frequency range, which leads to a sharpening of the frequency tuning of LBN1 as compared to AN1 (Figure 44B). 

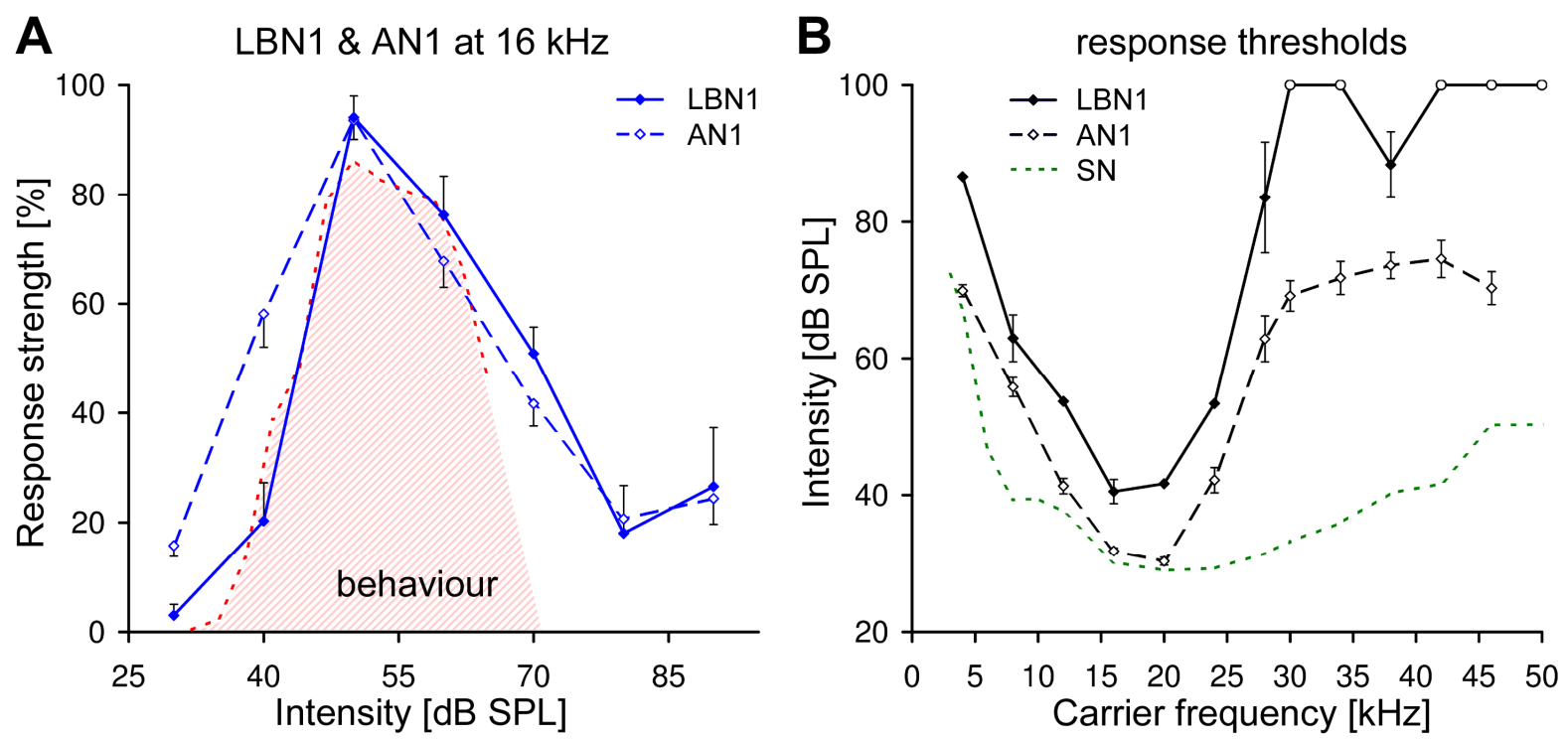

Figure 44: Responses of LBN1 and AN1 to stimuli with varying carrier frequency and intensity. A: Comparison of LBN1's and AN1's response strength at $16 \mathrm{kHz}$ and increasing intensities to the female behaviour (red shaded area). B: Response thresholds at various frequencies (4 to $50 \mathrm{kHz}$ ) for LBN1 ( $N=5-6$ for $8,16,28$ and $38 \mathrm{kHz}$; means and $\mathrm{SE}$, at other frequencies $\mathrm{N}=1$ with $\mathrm{n}=5$ ), AN1 $(\mathrm{N}=10-23$; means and $\mathrm{SE}$; taken from Stumpner 1997) and various sensory neurons (SN). If no response to a stimulus up to $90 \mathrm{~dB}$ SPL occurred, the threshold was set to be $100 \mathrm{~dB}$ SPL (data points indicated as open circles for LBN1 - the natural values might be even higher).

\subsubsection{Local brain neuron 2 (LBN2)}

Another local brain neuron that can be frequently found is LBN2. Because of its spontaneous activity and characteristical response to acoustic stimuli it can be easily identified during the intracellular recording.

Morphology. LBN2 has been stained in four individuals and the morphology is shown in Figure 45A, B. LBN2's spatial extent is restricted to one half of the brain. Its soma is located close to the lateral anterior surface of the brain and to the ventral beginning of the optical lobe. The primary neurite runs into posterior and more ventral portions of the brain and gives off a major branch to medial areas. This branch arborises strongly and the majority of the endings have a fine structure indicating to be of dendritic origin (asterisk in Figure 45B right). The main stem runs posterior and splits into two axons. One branch extends laterally and arborises strongly. The other branch extends medially and gives off a few tiny branches. Then it takes course to posterior lateral portions and divides into two branches. One branch is running to medial dorsal portions along the posterior border of the lateral protocerebrum and gives off dense arborisations. The other branch extends to anterior dorsal portions 
along the lateral border of the lateral protocerebrum and then to medial portions. During this course it arborises densely. The arborisations of the latter described axonal branches cover nearly the whole posterior and lateral border of the lateral protocerebrum in a more or less thin layer. All arborisations of the axon have beaded endings and therewith, indicate to be the major output site of LBN2.

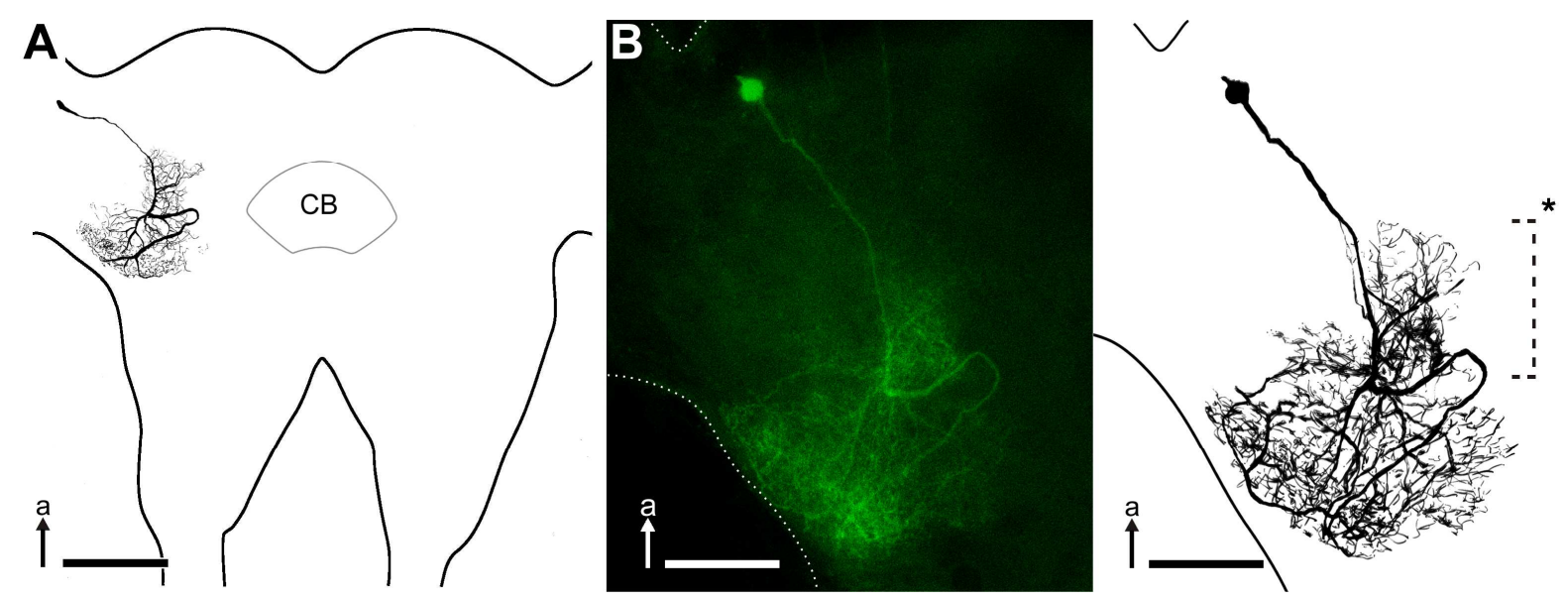

Figure 45: Morphology of LBN2 in the brain of $A$. nigrovittata. A: Drawing of LBN2 after a Cy3-staining in relation to the central body (CB). Bar represents $250 \mu \mathrm{m}$. B: Left: Confocal picture of a FITC stained LBN2 from a different individual than shown in A. Right: Drawing of the FITC stained LBN2 (seen left) at a larger magnification. The asterisk marks the parts of the neuron with a dendritic appearance. Bar represents $100 \mu \mathrm{m}$. $\mathrm{a}=$ anterior.

Intensity and carrier frequency dependence of LBN2 responses. In an example recording of LBN2 to an artificial duet, a strong spiking response to the male song at $16 \mathrm{kHz}$ and also to the female reply at $28 \mathrm{kHz}$ can be seen (Figure 46A upper trace). A post-excitatory inhibition of the spontaneous activity can be observed subsequent to the male's pulse series. Without stimulation LBN2 shows high spontaneous activity that has been observed in every single recording of LBN2 in this study (Figure 46A lower trace). This spontaneity of spiking shows no obvious correlation to a specific internal oscillation or a dependence on physiological patterns as for example the breathing rhythm. The responses to standard stimuli are displayed in Figure 46B. Due to the high intraindividual variability of total spike numbers only the relative magnitude of LBN2 responses is given. The high spontaneous spiking activity can also be observed when looking at $30 \mathrm{~dB}$ SPL stimuli (a $50 \%$ response equals approximately 4 spikes). 

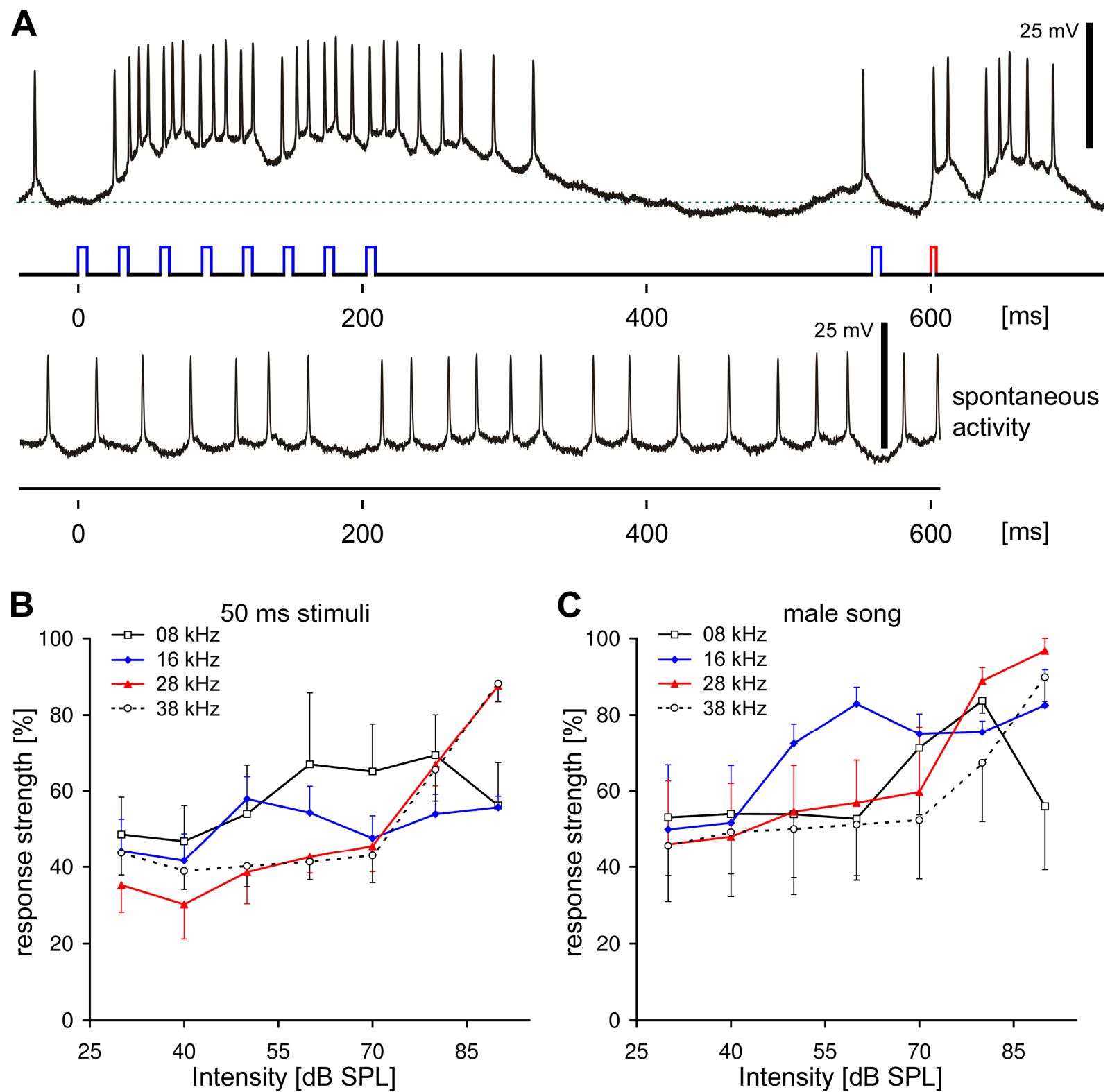

Figure 46: Responses of LBN2 to acoustic stimuli. Blue colouration - carrier frequency of the male song $(16 \mathrm{kHz})$ and red colouration - carrier of the female reply $(28 \mathrm{kHz})$. A: Upper trace: Example recording of the response to an artificial duet of $A$. nigrovittata (male song at $70 \mathrm{~dB}$ SPL; female answer at $60 \mathrm{~dB}$ SPL; see chapter 2.3.2 for stimulus paradigm). Lower trace: Example recording of LBN2's spontaneous activity without stimulation. B: Response of LBN2 to standard stimuli (see chapter 2.3.2) ( $\mathrm{N}=4$; means and $\mathrm{SE})$. C: Intensity dependent response to the male song pattern at 8 , 16, 28 and $38 \mathrm{kHz}$ ( $\mathrm{N}=3$; see chapter 2.3.2 for stimulus paradigm; means and $\mathrm{SE}$ ).

The lowest thresholds of LBN2's responses are found for 8 and $16 \mathrm{kHz}$. Whereas the spiking activity to $16 \mathrm{kHz}$ reaches a first maximum at $50 \mathrm{~dB} \mathrm{SPL}$, slightly decreases to $70 \mathrm{~dB}$ SPL and then increases again to $90 \mathrm{~dB}$ SPL. Responses to $8 \mathrm{kHz}$ are maximal between 60 and $80 \mathrm{~dB}$ SPL. The spiking activity to ultrasonic frequencies, on the other hand, is steeply increasing at intensities of 80 and $90 \mathrm{~dB}$ SPL. When presenting the temporal structure of the male song (at the same intensities and frequencies) (Figure 46C; a 50\% response equals approximately 18 spikes) these 
characteristics of LBN2's response slightly differ from the results obtained with $50 \mathrm{~ms}$ stimuli (compare to Figure 46B). The most obvious difference is found for the responses at $8 \mathrm{kHz}$. The threshold increases to $70 \mathrm{~dB}$ SPL and the spiking activity is strongly reduced at $90 \mathrm{~dB} \mathrm{SPL}$. Also a stronger response to $28 \mathrm{kHz}$ at $80 \mathrm{~dB} \mathrm{SPL}$ can be observed. Comparing the responses to $16 \mathrm{kHz}$ at $60 \mathrm{~dB}$ SPL with the other carrier frequencies a higher activity of approximately 30 to $40 \%$ can be seen. The response strength at ca. $50 \%$ shows high standard errors which are mostly due to the spontaneous activity. Activity above ca. $70 \%$ shows the smallest error. However, test programs with stimuli consisting of 50 ms pulses or male song models at various frequencies and intensities cannot explain the strong response of LBN2 to the female reply as shown in Figure 46A (intensity of female answer pulse is $60 \mathrm{~dB}$ SPL).

Responses to different frequencies of the female answer in an artificial duet. An evaluation of LBN2's response to the female reply only (for various frequencies at $60 \mathrm{~dB} \mathrm{SPL}$ ) is shown in Figure 47A. An increased spiking activity can only be observed at 16, 24 and $28 \mathrm{kHz}$ with responses to $28 \mathrm{kHz}$ being the strongest. Notably, the carrier frequency dependent response exhibits 2 maxima, which results from a consistently decreased response to $20 \mathrm{kHz}$, despite the high standard errors.
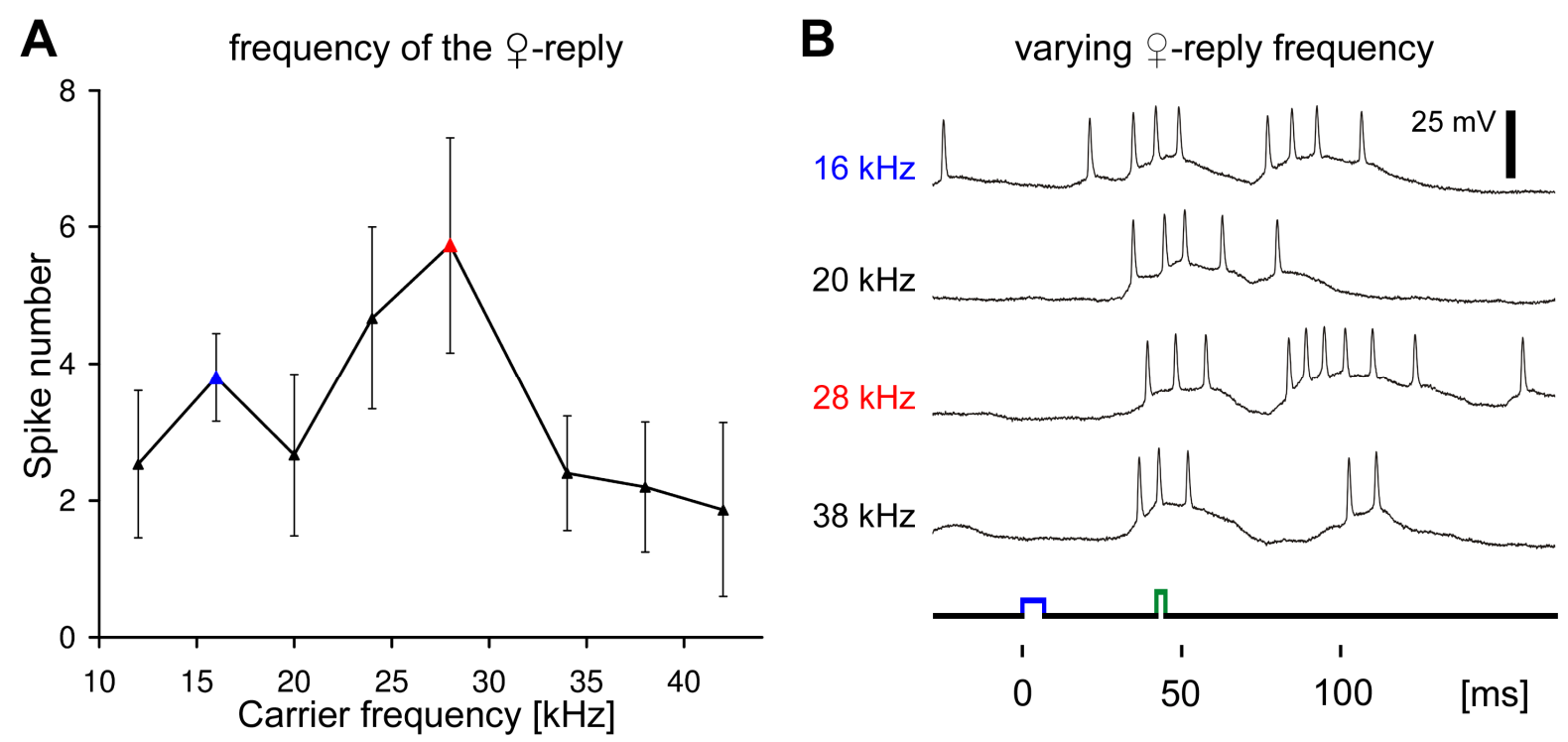

Figure 47: Response of LBN2 to the female answer with varying carrier frequency in an artificial duet (see chapter 2.3.2 for stimulus paradigm). Blue indicates the male's natural carrier frequency and red the natural frequency of the female reply. A: Spike numbers of LBN2 ( $N=3$; means and SE). B: Recording examples of LBN2's response to the female reply at 16, 20, 28 and $38 \mathrm{kHz}$. Note the decreased response to the female reply at 20 and $38 \mathrm{kHz}$. 
A recording example of one individual is given in Figure 47B. Both, at 16 and $28 \mathrm{kHz}$ a strong graded potential with action potentials riding on the top caused by the female answer can be observed. At $20 \mathrm{kHz}$ a major response in LBN2 occurs to the male trigger only. At $38 \mathrm{kHz}$ the response to the female reply is considerably lower than at 16 or $28 \mathrm{kHz}$.

To test, whether this increased response to the female reply at the correct carrier frequency of $28 \mathrm{kHz}$ only occurs when the male trigger pulse is closely preceding, an artificial duet with variations in the pause duration between trigger and female answer was presented (Figure 48). At pause durations of $85 \mathrm{~ms}$ - a distance where graded potentials generated to the male trigger do not interfere with the response to the female reply - a strong burst of action potentials is elicited to the female reply. This shows the independency of the response to the female's reply of the preceding trigger. The shorter pause durations get, the lower the response to the females reply. At pause durations of $5 \mathrm{~ms}$ a response is elicited to the male trigger only. A possible cause might be the post-excitatory inhibition (see Figure 46A) that occurs subsequent to the male trigger.

varying delay of the $q$-reply

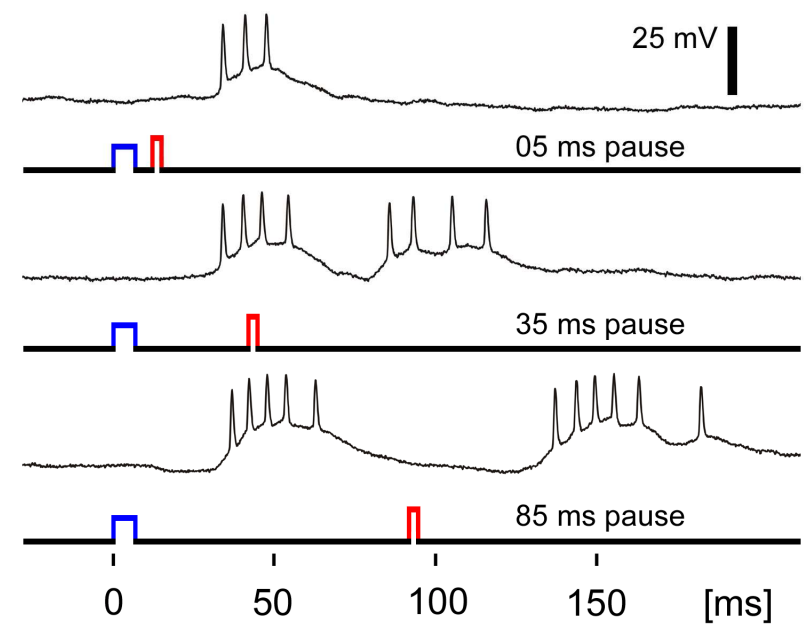

Figure 48: Recording examples of LBN2's response to the female answer (at $28 \mathrm{kHz}$ ) with varying pause duration between male trigger and female reply in an artificial duet $(5,35$, and $85 \mathrm{~ms}$ pause duration; see chapter 2.3.2 for stimulus paradigm). Blue indicates the male's natural carrier frequency and red the natural frequency of the female reply.

Carrier frequency dependent IPSPS in LBN2. During stimulation with varying carrier frequency and increasing intensity excitation of LBN2 at sonic frequencies (e.g. $16 \mathrm{kHz}$ at $60 \mathrm{~dB}$ SPL) and at ultrasonic frequencies (e.g. 28 and $38 \mathrm{kHz} 90 \mathrm{~dB}$ SPL) can be observed (Figure 49). In addition, acoustically evoked inhibition of LBN2 can be seen for ultrasonic stimuli preceding the excitation (arrows in Figure 49). Typically, these IPSPs can be clearly observed at high intensities only (80 and $90 \mathrm{~dB}$ SPL). With positive current injection the lowest thresholds of these IPSPs were 
observed at approximately 60 to $70 \mathrm{~dB}$ SPL. The IPSP always precedes the EPSP and has a shortest mean latency of $24.0 \pm 0.5 \mathrm{~ms}(\mathrm{~N}=4$; at $28 \mathrm{kHz}, 80 \mathrm{~dB}$ SPL; mean and SE). This may indicate a possible direct connection to an ascending interneuron since shortest spike latencies for e.g. AN2 are $21.7 \mathrm{~ms}$ (see chapter 3.1.2). For comparison the EPSP at the same frequency and intensity has a mean latency of $35.0 \pm 1.4 \mathrm{~ms}(\mathrm{~N}=4$; mean and $\mathrm{SE}$ ). The beginning of the EPSP at ultrasound, however, is always affected by the preceding IPSP. Thus, shortest mean EPSP latencies can be obtained at $16 \mathrm{kHz}$ where no initial IPSP is present, with $32.2 \pm 1.2 \mathrm{~ms}$ ( $60 \mathrm{~dB}$ SPL; $\mathrm{N}=3$; mean and SE). According to this, in the excitatory pathway of LBN2 at least one additional brain neuron has to be involved.

\section{Frequency dependent IPSP in LBN2}

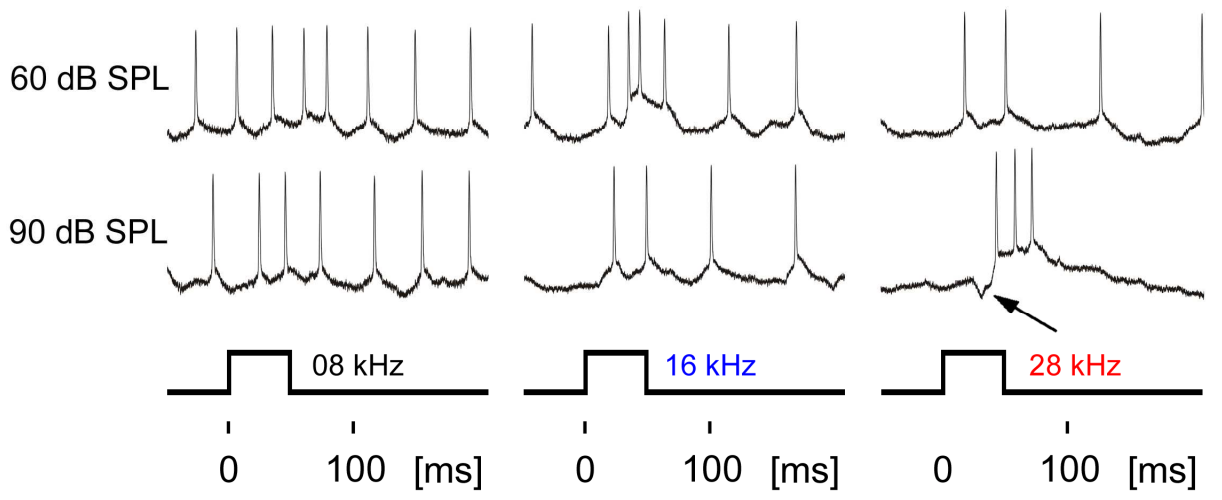

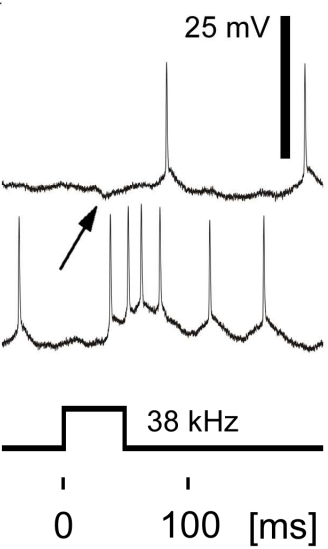

Figure 49: Recording examples of LBN2 to standard stimuli at 60 and $90 \mathrm{~dB}$ SPL. The arrows mark the preceding IPSP seen at ultrasonic stimulation.

Response of LBN2 to varying temporal parameters. LBN2's response to a presentation of white noise with variations in the pulse/pause patterns is displayed in Figure 50. For differing pause durations the strongest response can be observed for the shortest as well as to longest durations tested (Figure 50A). The least response is revealed to the natural male song pause duration $(22 \mathrm{~ms})$. For comparison, although the level of LBN2's spontaneous activity is displayed $(66.7 \pm 17.8 \%(\mathrm{~N}=3$; mean and SE), grey line in Figure 50A). A comparison to the specificity of the female behaviour reveals an inverse correlation with LBN2's spike activity. Hence, the response of LBN2 may contribute to the female behaviour if the connection to its postsynaptic neuron would be inhibitory. Stimulation with varying pulse durations reveals the lowest activity close to the rate of spontaneous activity $(65.7 \pm 11.7 \%$ $(\mathrm{N}=3$; mean and $\mathrm{SE})$, grey line in Figure $50 \mathrm{~B})$ for the shortest pulse duration tested. In contrast, the spiking activity is highest to pulses of $7 \mathrm{~ms}$ and longer. Contrary to 
the inverse correlation found for varying pause durations, LBN2's spike activity parallels the behaviour of females to shorter pulse durations than $7 \mathrm{~ms}$.

A

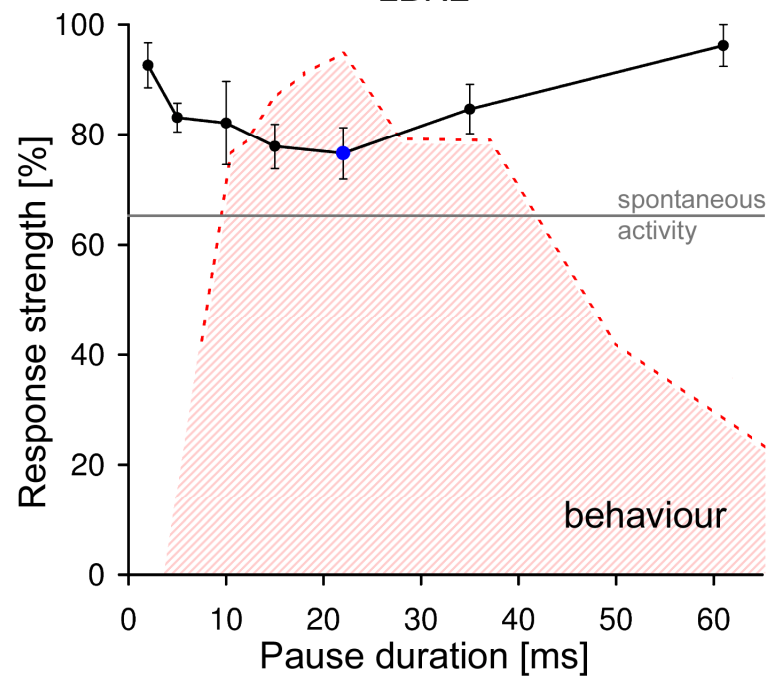

B

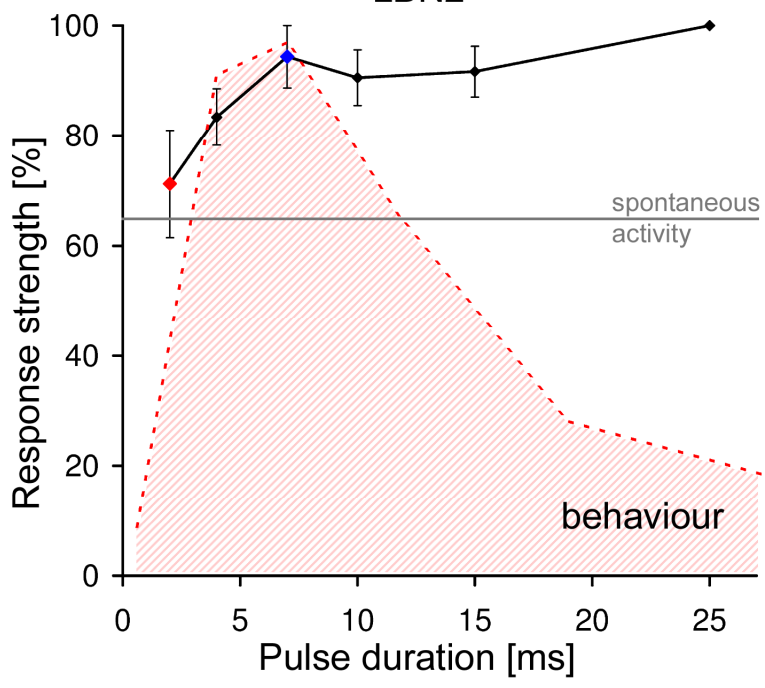

Figure 50: Mean responses of LBN2 to different temporal parameters (white noise; $70 \mathrm{~dB}$ SPL; see chapter 2.3.2 for stimulus paradigm; means and SE). Important natural parameters are emphasised (blue: male song pause/pulse duration; red: female reply pulse duration). Red shaded area indicates female behaviour ( $N=5$, modified from Dobler et al. 1994a). Grey line marks the level of spontaneous activity (mean). A: Magnitude of response to stimuli with varying pause duration $(\mathrm{N}=3)$. $\mathbf{B}$ : Magnitude of response to stimuli with varying pulse duration $(\mathrm{N}=3)$.

Dependence on pulse duration of the IPSP in LBN2. During stimulation with white noise stimuli of varying pulse durations one can see that LBN2 gets multiple inhibitory input (Figure 51). The above mentioned, initial IPSP is not seen prior to the excitation, but at $2 \mathrm{~ms}$ pulses an inhibition of spontaneous activity can be observed subsequent to a short burst of spikes.

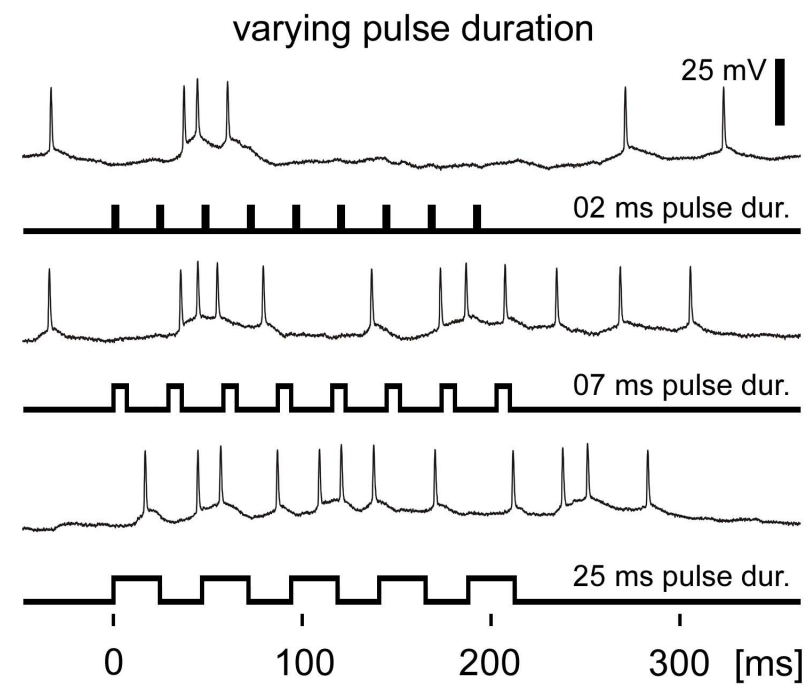

Figure 51: Recording examples of LBN2 to white noise stimuli (70 dB SPL) with 2, 7 and $25 \mathrm{~ms}$ pulse duration (see chapter 2.3 .2 for stimulus paradigm). Note the inhibition of spontaneous activity of the response at $2 \mathrm{~ms}$ pulse duration. 
Stimulation with pulse durations of $25 \mathrm{~ms}$ elicits a clear response of LBN2, whereas the spike activity is only slightly reduced when pulses are shortened to the natural male song pulse duration of $7 \mathrm{~ms}$. This indicates that the inhibitory influence in LBN2 has the ability to effectively lower the excitation, when series of short pulses are presented (see also Figure 50B).

Spike latencies in LBN2. The shortest spike latencies in LBN2 were observed for $50 \mathrm{~ms}$ pulses at $16 \mathrm{kHz}$ at $60 \mathrm{~dB}$ SPL with $37.1 \pm 2.0 \mathrm{~ms}$ ( $\mathrm{N}=4$; mean and $\mathrm{SE}$ ). At the same carrier frequency and intensity also shortest latencies were found when the male song paradigm was presented.

In summary, LBN2 is influenced by at least two, possibly three auditory neurons. The IPSP - which is strongest at ultrasonic frequencies and short pulse durations - has short latencies and may be directly caused by an ascending interneuron. The EPSP has long latencies, which is true for sonic and ultrasonic frequencies. The characteristics of the excitation for low intensities are firstly, strong responses to $16 \mathrm{kHz}$ stimuli that are independent of stimulus duration (see Figure 46B \& Figure 47B) and secondly, bursts of action potentials that occur strongest to short pulses only. These response characteristics combined in a single neuron have not been observed in any ascending neuron. Therefore, at least two different ascending neurons must be involved in the excitatory pathway to LBN2. Whether this combined response is fed into LBN2 via a single presynaptic local brain neuron cannot be decided from the data of this study.

\subsubsection{Local brain neuron 3 (LBN3)}

LBN3 is an auditory brain neuron that has only been recorded twice. Here, the data of one LBN3 will be exemplarily presented. The LBN3 recorded in a different specimen behaved accordingly.

Intensity and carrier frequency dependence of $\angle B N 3$ responses. Presentation of an artificial duet evoked action potentials in LBN3 to both, the calling song of the male and to the female reply (Figure $52 \mathrm{~A}$; due to the low recording quality a $5000 \mathrm{~Hz}$ low-pass filter was applied). In the upper trace a single recording is displayed, whereby the lower trace shows an average of LBN3's responses. The averaged 
EPSPs in LBN3 closely follow the pulse pattern of the male song. Also the graded responses to the trigger pulse and the female reply are clearly separated. This characteristic, however, is not apparent in the single recording (upper trace). The spiking activity of LBN3 to standard stimuli shows lowest thresholds for 16 and $28 \mathrm{kHz}$ (Figure 52B). The strongest activity can be seen for ultrasonic frequencies at $90 \mathrm{~dB}$ SPL. The response of LBN3 to an artificial duet with varying carrier frequency of the female reply is shown in Figure 52C. The strongest responses are revealed for 24 and $28 \mathrm{kHz}$. The female answer at $16 \mathrm{kHz}$ did not elicit a single spike in 5 repetitions.
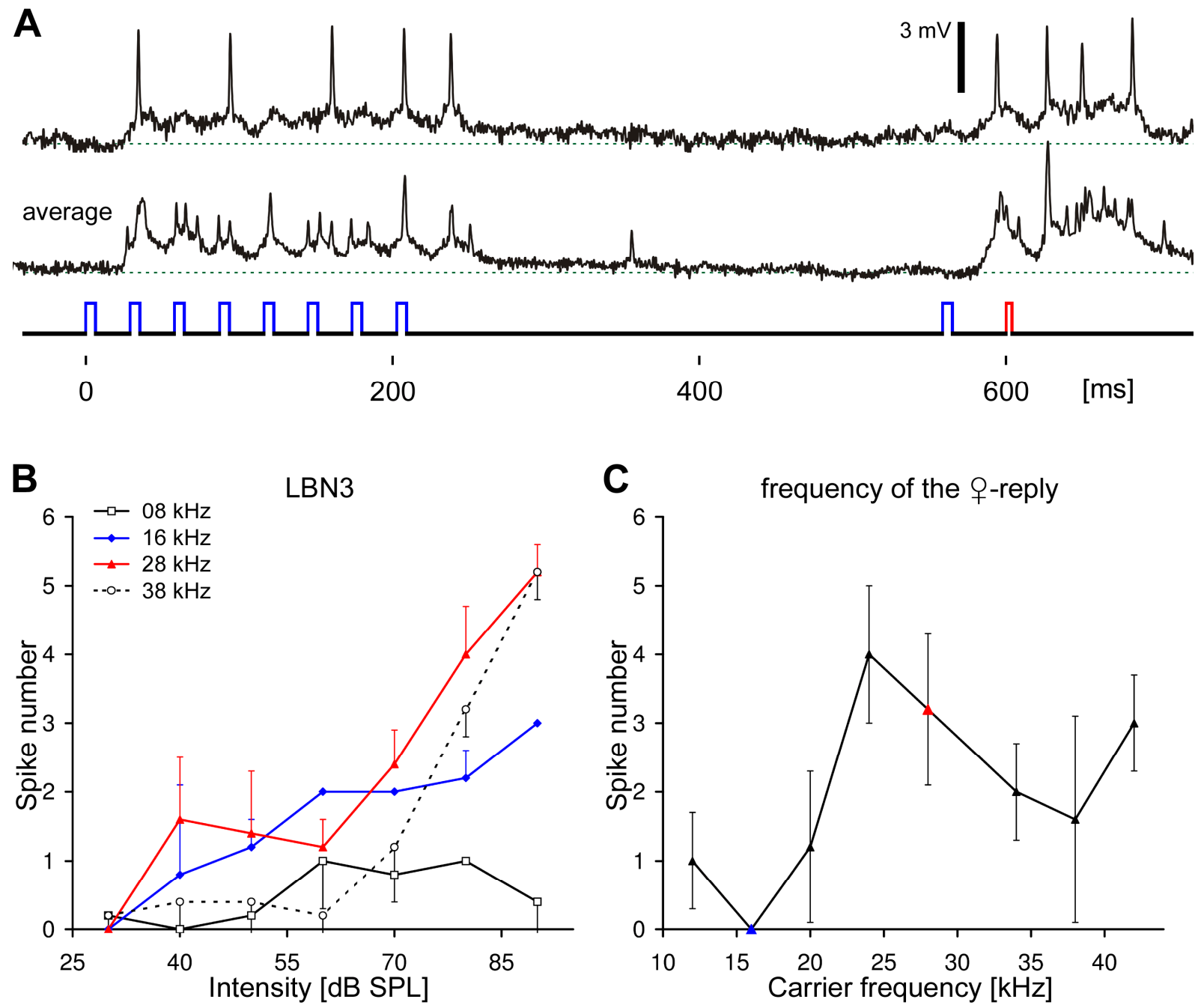

Figure 52: Response of LBN3 to acoustic stimuli. Blue colour - carrier frequency of the male song $(16 \mathrm{kHz})$ and red colour - carrier of the female reply $(28 \mathrm{kHz})$. A: Example recordings of the response to an artificial duet (male song at $70 \mathrm{~dB}$ SPL; female answer at $60 \mathrm{~dB}$ SPL; see chapter 2.3.2 for stimulus paradigm; filtered with a $5000 \mathrm{~Hz}$ low-pass). Upper trace shows a single recording whereas the lower trace shows an average of 5 repetitions. B: Response of LBN3 to standard stimuli (see chapter 2.3.2) ( $n=5$; means and SD). C: Response to the female answer with varying carrier frequency in an artificial duet ( $n=5$; see chapter 2.3.2 for stimulus paradigm; means and SD). 
Latency of $\angle B N 3$ and the connection to ascending interneurons. Although the morphology of LBN3 is unknown, the existence of prominent EPSPS (see Figure 52A) reveal LBN3 to be an interneuron that gets its input in the brain. Shortest spike latencies are found for $16 \mathrm{kHz}$ at $90 \mathrm{~dB}$ SPL with $26.8 \pm 2.2 \mathrm{~ms}(\mathrm{~N}=2, \mathrm{n}=10$; mean and SD). The EPSP, however, has a mean latency of $19.0 \pm 1.5 \mathrm{~ms}(\mathrm{~N}=2, \mathrm{n}=9$; mean and SD) at the same parameters, which is consistent with LBN3 being directly connected with an ascending interneuron. Comparing the spiking activity of LBN3 to standard stimuli with the response of AN3 reveals some similarities (Figure 53A, B). Both neurons comprise lowest response thresholds for 16 and $28 \mathrm{kHz}$, and stimuli with $8 \mathrm{kHz}$ elicit the least spike numbers. Also, in both neurons the responses to $16 \mathrm{kHz}$ appear to saturate at $60 \mathrm{~dB}$ SPL before they increase again at $90 \mathrm{~dB}$ SPL. The latencies of AN3 to $16 \mathrm{kHz}$ stimuli at $90 \mathrm{~dB}$ SPL are $19.4 \pm 0.5 \mathrm{~ms}(\mathrm{~N}=9$; mean and SE), which makes a direct connection between both neurons possible, although an approximate synaptic delay is reported to be in the order of 1-2 ms (Hennig 1988). In this case, however, one has to keep in mind that the above mentioned, identical latencies were obtained in different individuals and that the variability of the EPSP latency in LBN3 is comparatively high.

A

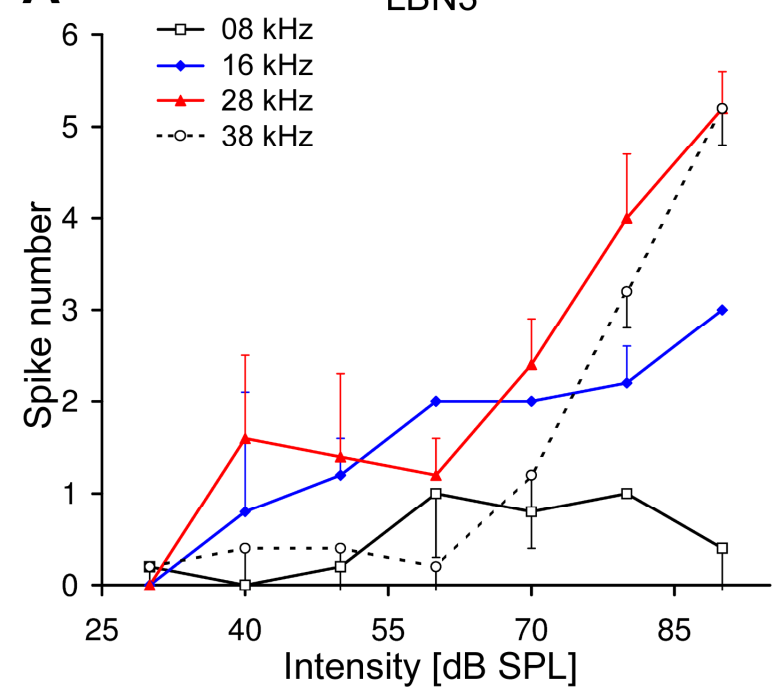

B

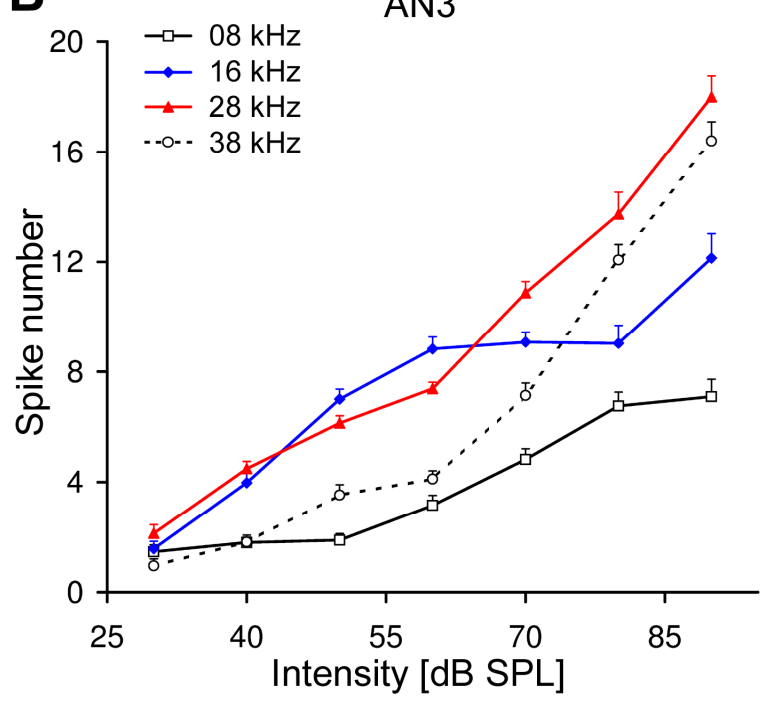

Figure 53: Response of LBN3 and AN3 to standard stimuli (see chapter 2.3.2). Blue colour - carrier frequency of the male song $(16 \mathrm{kHz})$ and red colour - carrier of the female reply $(28 \mathrm{kHz})$. A: Response of LBN3 ( $n=5$; means and SD). B: Response of AN3 ( $N=9$; means and SE).

Additional indication for LBN3 being postsynaptic to AN3 is the tonic response to stimuli with long durations. In Figure 54 recording examples of the responses of AN3 and LBN3 is displayed to a 500 ms white noise pulse (70 dB SPL). In AN3 a strong and tonic response can be seen that closely copies the whole length of the 
stimulus. This also reflects the amplitude of the EPSP in LBN3, whereas distinctly fewer spikes are elicited.

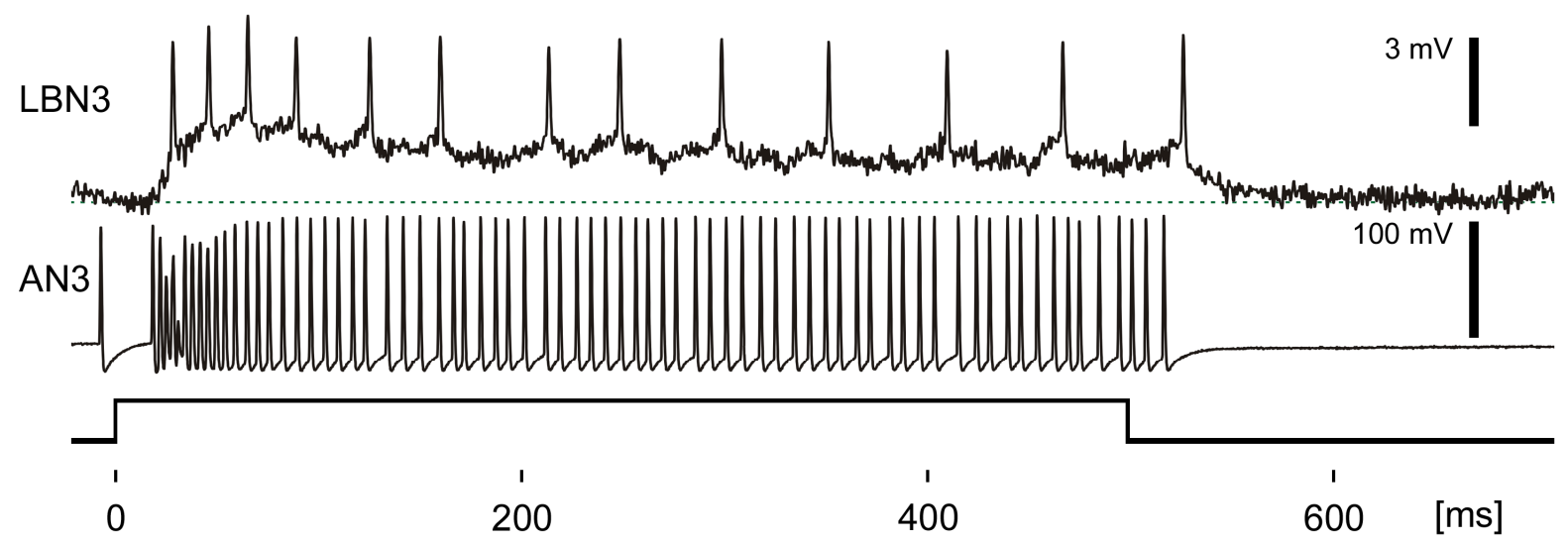

Figure 54: Example recordings of LBN3 (low-pass filtered at $5000 \mathrm{~Hz}$ ) and AN3 to a $500 \mathrm{~ms}$ white noise pulse (at $70 \mathrm{~dB} S P L)$.

A closer look on averaged responses of LBN3 to standard stimuli does not only reveal excitatory postsynaptic potentials (Figure 55). Stimulation with ultrasonic frequencies at high intensities also shows inhibitory input decreasing the response of LBN3 (marked by the asterisks).

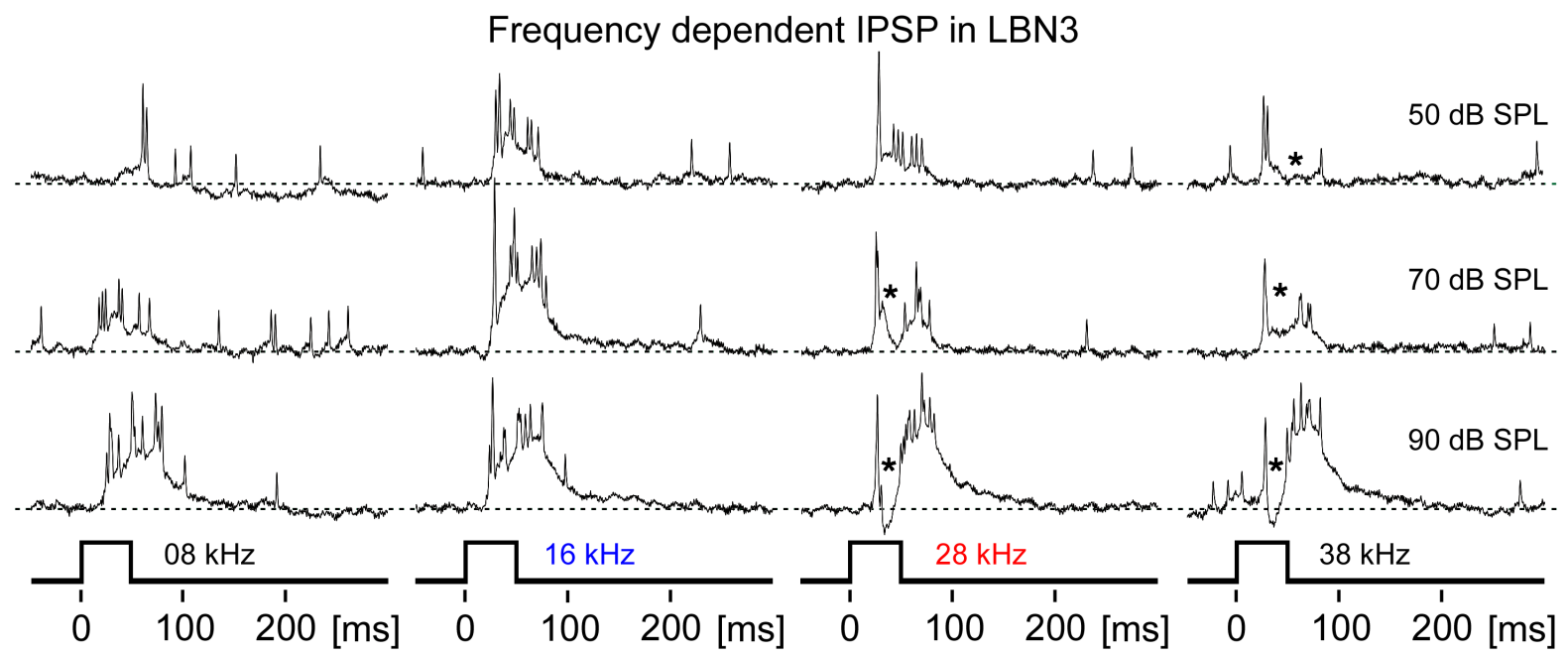

Figure 55: Averaged recording examples of LBN3 (low-pass filtered at $5000 \mathrm{~Hz}$ ) to standard stimuli at 50,70 and $90 \mathrm{~dB}$ SPL. The asterisks mark a clear reduction of LBN3's response due to inhibition.

This inhibition, subsequent to the excitation, is frequency dependent and also seems to affect the spiking activity at low intensities of 28 and $38 \mathrm{kHz}$ (e.g. the apparently reduced spiking activity at $38 \mathrm{kHz} 50 \mathrm{~dB} \mathrm{SPL}$ ). The inhibition of response to ultrasonic frequencies is also reflected by LBN3's spiking activity displayed in Figure 53A. It seems to strongly reduce the activity at 50 to $70 \mathrm{~dB} \mathrm{SPL}$ (compared to 
the response of AN3 in Figure 53B). At higher intensities excitation obviously overcomes the inhibition by a tonic EPSP following the IPSP (Figure 55). The latency of the IPSP could not be measured since the excitation concealed the beginning of the inhibition. But the IPSP seems to stem from a neuron that exhibits a phasic response to $50 \mathrm{~ms}$ stimuli since the duration of the IPSP is much shorter than the simultaneous excitation.

\subsubsection{Local brain neuron 4 (LBN4)}

LBN4 is an interneuron that has only been recorded once. Nevertheless, the physiological data of LBN4 will be presented in this chapter, since its unique response provides a deeper insight in the neuronal processing of auditory stimuli and possible connections between interneurons in the brain of $A$. nigrovittata.

Morphology. LBN4 is restricted to one side of the brain (Figure 56A). Its soma is situated in dorsal lateral portions close to the posterior entrance of the optic lobe. The primary neurite runs posterior and then slightly medial ventral, where a single branch splits off that extends dorsally with minor arborisations.

A

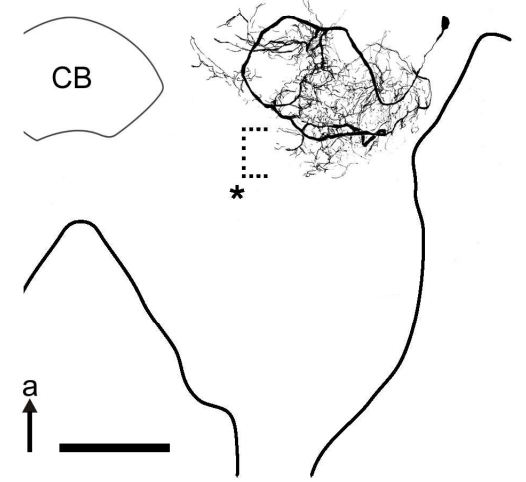

B

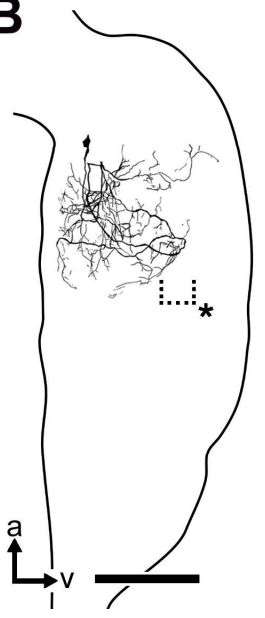

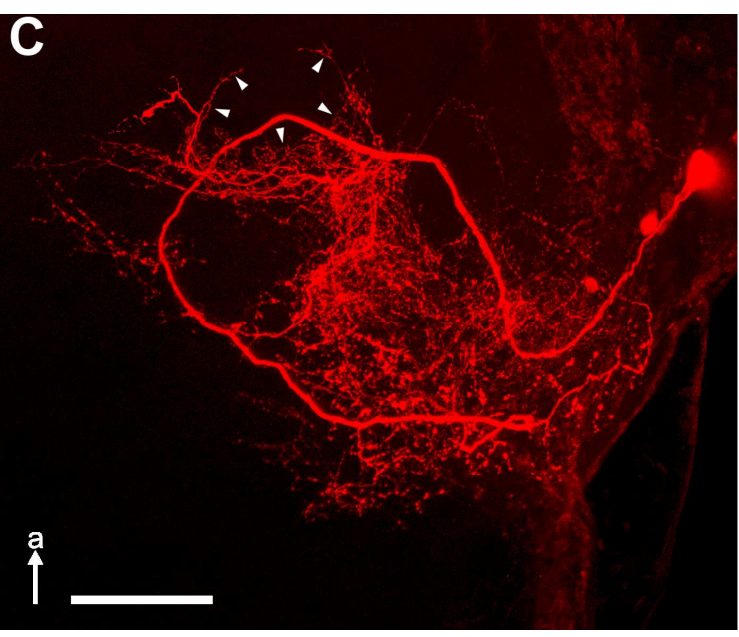

Figure 56: Morphology of LBN4 in the brain of $A$. nigrovittata. A: Drawing of LBN4 after a Cy3-staining in relation to the central body (CB). Bar represents $200 \mu \mathrm{m}$. The asterisk marks the parts of the neuron with axonic appearance. B: Sagittal view on LBN3. Bar represents $200 \mu \mathrm{m}$. The asterisk marks the parts of the neuron with axonic appearance. C: Detailed view on LBN3 of the Cy3-staining. The arrowheads mark arborisations in a "U" shape. Bar represents $100 \mu \mathrm{m}$. $\mathrm{a}=$ =anterior, v=ventral.

The main stem continues first to anterior, then to medial and subsequently to posterior portions of the brain. In the part that extends medially two branches split off. One branch extends to dorsal portions and arborises. The other branch runs to 
medial ventral portions close to the ventral perineurium of the brain. On its way ramifications are found that remind of a "U" shape (marked by the arrowheads in Figure 56C). A similar "U" shape can also be seen at the axonal branches of LBN1 (Figure 35B). However, the main stem continues to posterior ventral portions with a few tiny collaterals. Then, the main stem of LBN4 changes direction to lateral ventral regions. Here, a branch splits off that runs to the dorsal surface and arborises. All arborisations described to this point appeared to have smooth endings indicating to be postsynaptic. At the most ventral lateral portion of the main stem a few tiny arborisations are found that appear to have beaded endings and may, therefore, be the output region of LBN4 (marked by an asterisk in Figure 56A, B). The axon subsequently changes direction to dorsal medial portions without extensive ramifications. At its turning point, though, also a minor branch splits off that takes course to dorsal anterior portions along the lateral border of the protocerebrum. This branch also shows no extensive arborisations.

Intensity and carrier frequency dependence of LBN4 responses. Shortly after the beginning of the intracellular recording procedure, high spontaneous activity occurred in LBN4, which was most likely due to the penetration. Nevertheless, one can see the compound potentials in LBN4 provoked by an artificial duet (Figure 57A). In the upper trace large IPSPs are visible that closely follow the pulse pattern of the male calling song and also occur (slightly) to the female answer pulse. Additionally, one can observe a small EPSP preceding the IPSP. This is more obvious when taking the average of five responses (Figure 57A lower trace). Here one can see that the EPSPs occur at the beginning of the male's pulse series and at the beginning of the trigger pulse only. The spiking activity of LBN4 to standard stimuli, however, reveals no net increase at any intensity. Instead, a strong decrease of activity can be observed, which is due to the strong inhibition. The threshold for this inhibition is lowest for 16, 28 and $38 \mathrm{kHz}$ 

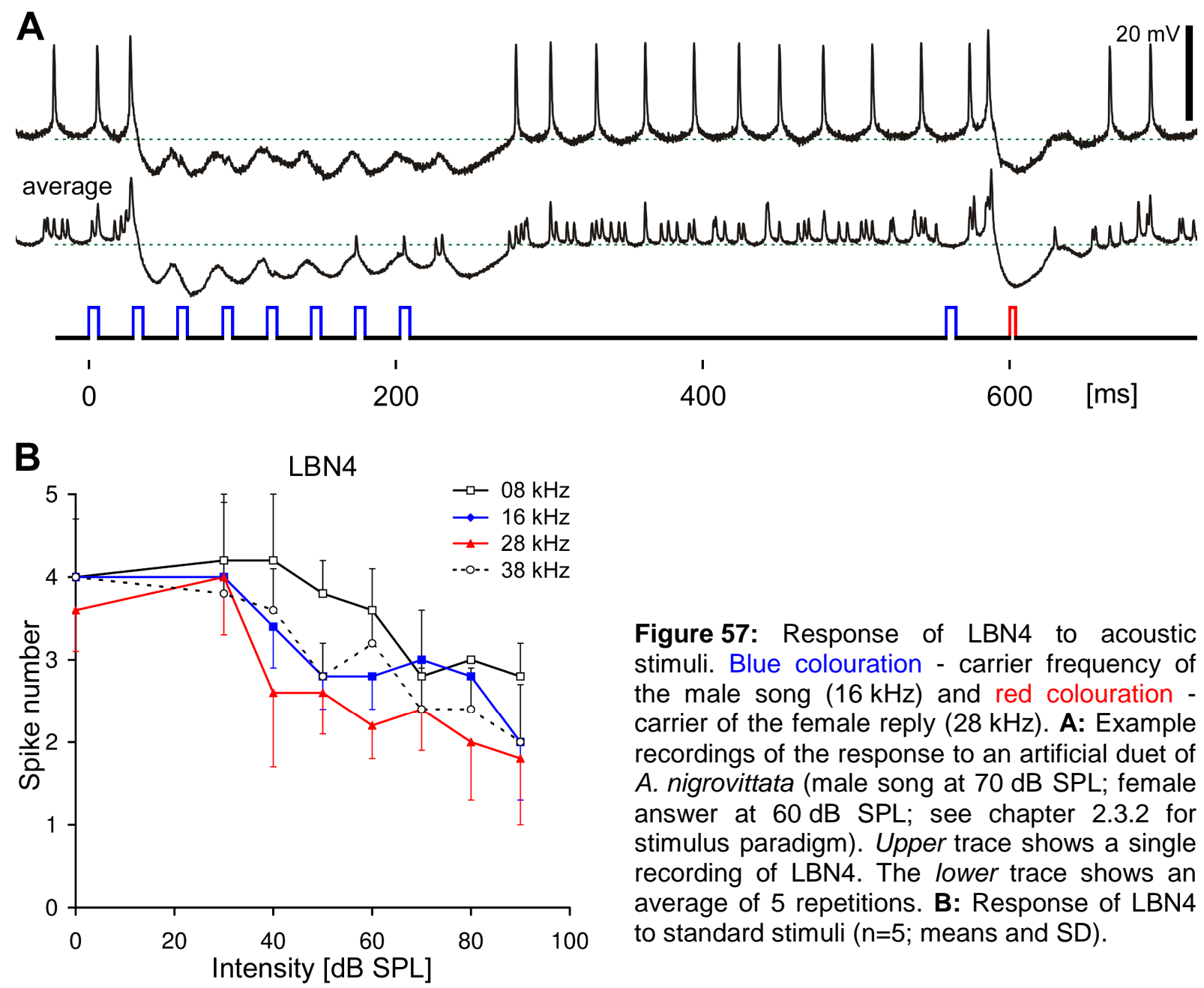

Figure 57: Response of LBN4 to acoustic stimuli. Blue colouration - carrier frequency of the male song $(16 \mathrm{kHz})$ and red colouration carrier of the female reply $(28 \mathrm{kHz})$. A: Example recordings of the response to an artificial duet of A. nigrovittata (male song at $70 \mathrm{~dB}$ SPL; female answer at $60 \mathrm{~dB}$ SPL; see chapter 2.3.2 for stimulus paradigm). Upper trace shows a single recording of LBN4. The lower trace shows an average of 5 repetitions. B: Response of LBN4 to standard stimuli ( $n=5$; means and SD).

Dependency of the IPSP on carrier frequency in LBN4. When stimulating with $50 \mathrm{~ms}$ pulses of the standard test the characteristics of the IPSP depending on carrier frequency become obvious (Figure 58). At low intensities (here: $60 \mathrm{~dB} S P L$ ) the IPSP is strongest for $16 \mathrm{kHz}$, whereas for higher and lower frequencies the IPSP strength is reduced. At high stimulus intensities this difference of IPSP strength between the carrier frequencies disappears. In the recording examples also the EPSP and a phasic response is clearly visible. At high intensities it can be seen at all frequencies tested, whereas at low intensities only responses to 8 and $16 \mathrm{kHz}$ show an EPSP. For one mean response of LBN4 (recording example at $16 \mathrm{kHz}, 90 \mathrm{~dB}$ $\mathrm{SPL}$ ) also single responses are given (grey recordings) to show the constancy of the IPSP amplitude of the single repetitions. 

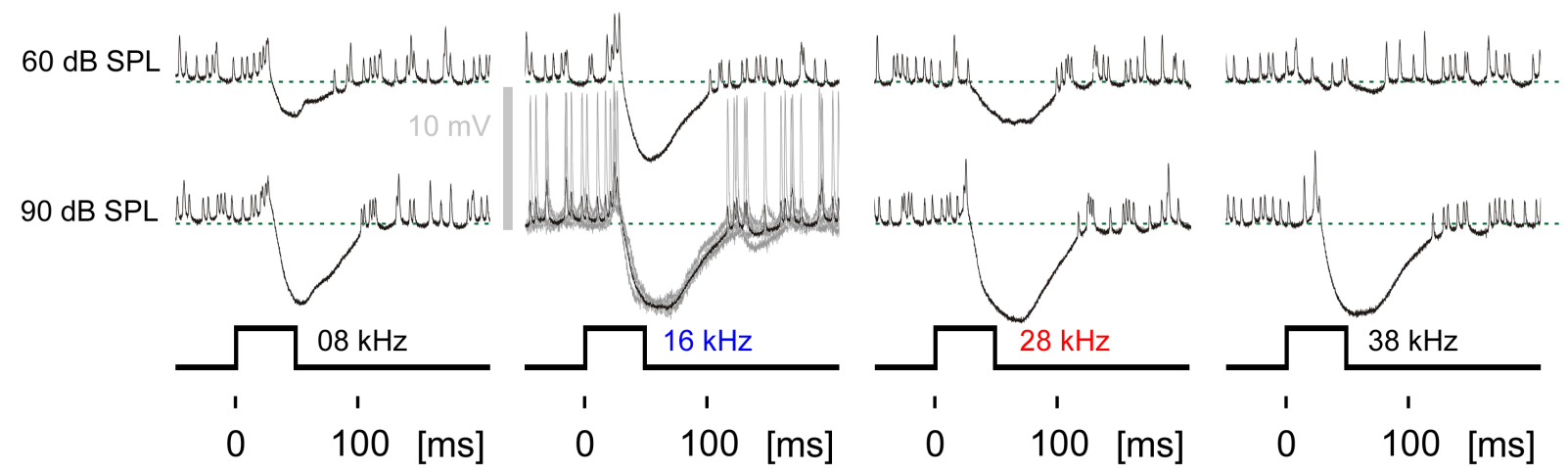

Figure 58: Averaged recordings (of 5 repetitions) of LBN4 to standard stimuli at 60 and $90 \mathrm{~dB}$ SPL. For $16 \mathrm{kHz}$ at $90 \mathrm{~dB}$ SPL also single responses are given to allow an estimation of the variability (grey recording). Blue colouration - carrier frequency of the male song $(16 \mathrm{kHz})$ and red colouration - carrier of the female reply $(28 \mathrm{kHz})$.

The relative IPSP amplitudes can be calculated from the mean responses of LBN4 (as displayed in Figure 58) by plotting the magnitude of IPSP amplitudes against increasing intensity for each frequency (Figure 59A). For low intensities (50 to $70 \mathrm{~dB} \mathrm{SPL}$ ) strongest IPSPs are found at $16 \mathrm{kHz}$ stimulation. At ultrasonic frequencies the IPSP amplitude first increases for $28 \mathrm{kHz}$ at $30 \mathrm{~dB}$ SPL (respectively for $38 \mathrm{kHz}$ at $40 \mathrm{~dB} \mathrm{SPL}$ ), then saturates until $70 \mathrm{~dB} \mathrm{SPL}$ and steeply increases to $90 \mathrm{~dB}$ SPL. Similar characteristics for the ultrasonic frequencies (and partly for $16 \mathrm{kHz}$ ) are found in the tonic spiking activity of LBN3 (Figure 59B).

A

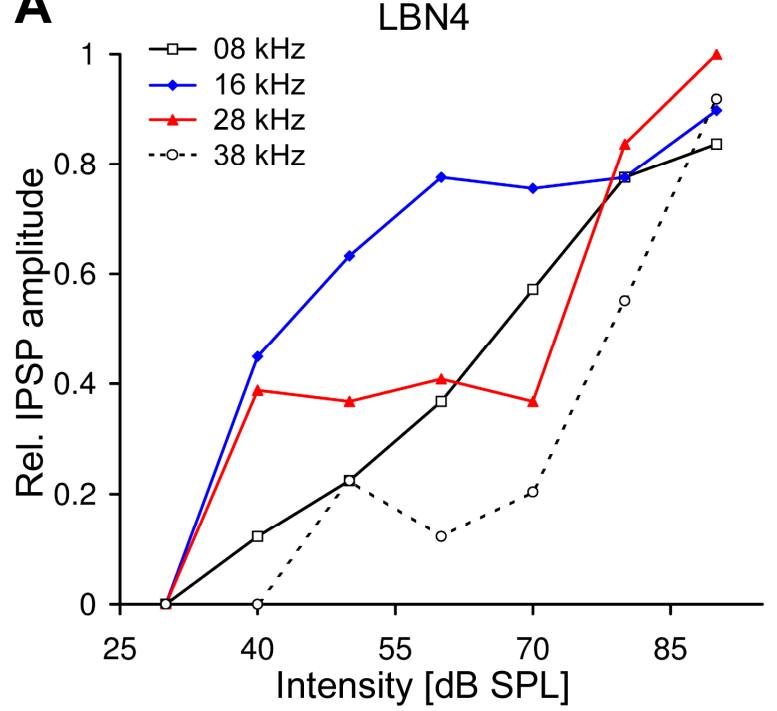

B

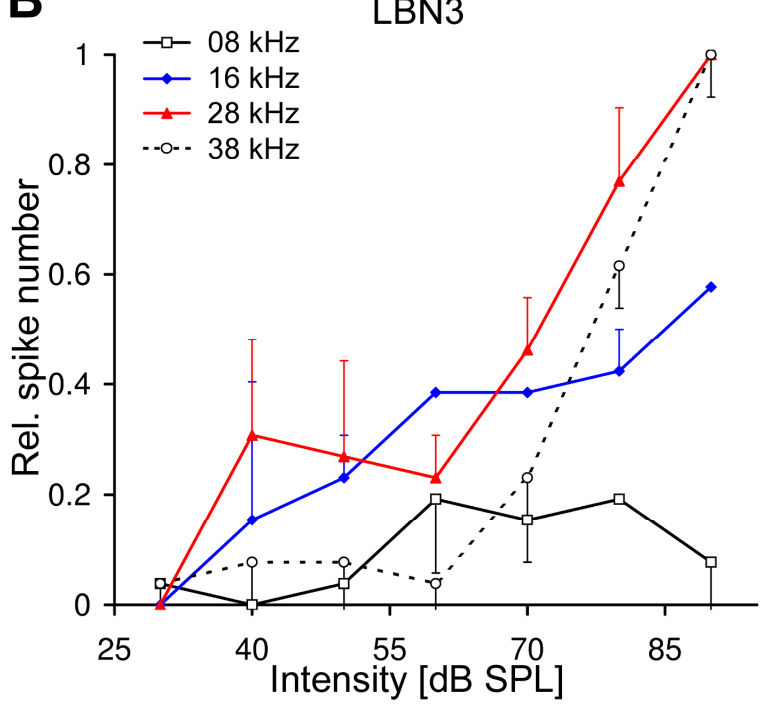

Figure 59: A: Relative amplitude of the IPSP in LBN4 calculated from the averaged responses $(n=5$; means). B: Relative spike numbers of $\operatorname{LBN3}(\mathrm{n}=5$; means and SD). Blue colouration - carrier frequency of the male song $(16 \mathrm{kHz})$ and red colouration - carrier of the female reply $(28 \mathrm{kHz})$. 
The response to $500 \mathrm{~ms}$ white noise stimuli reveals strong and tonic inhibitory input into LBN4 (Figure 60 upper trace). These tonic characteristics have also been found in the responses of LBN3 as well as in AN3. AN3 is, among all other ascending interneurons, the only neuron that shows such a strong activity that reflects the whole length of the stimulus. Thus, a coupling of LBN4 to AN3 (possibly via LBN3 - see below) may be likely.

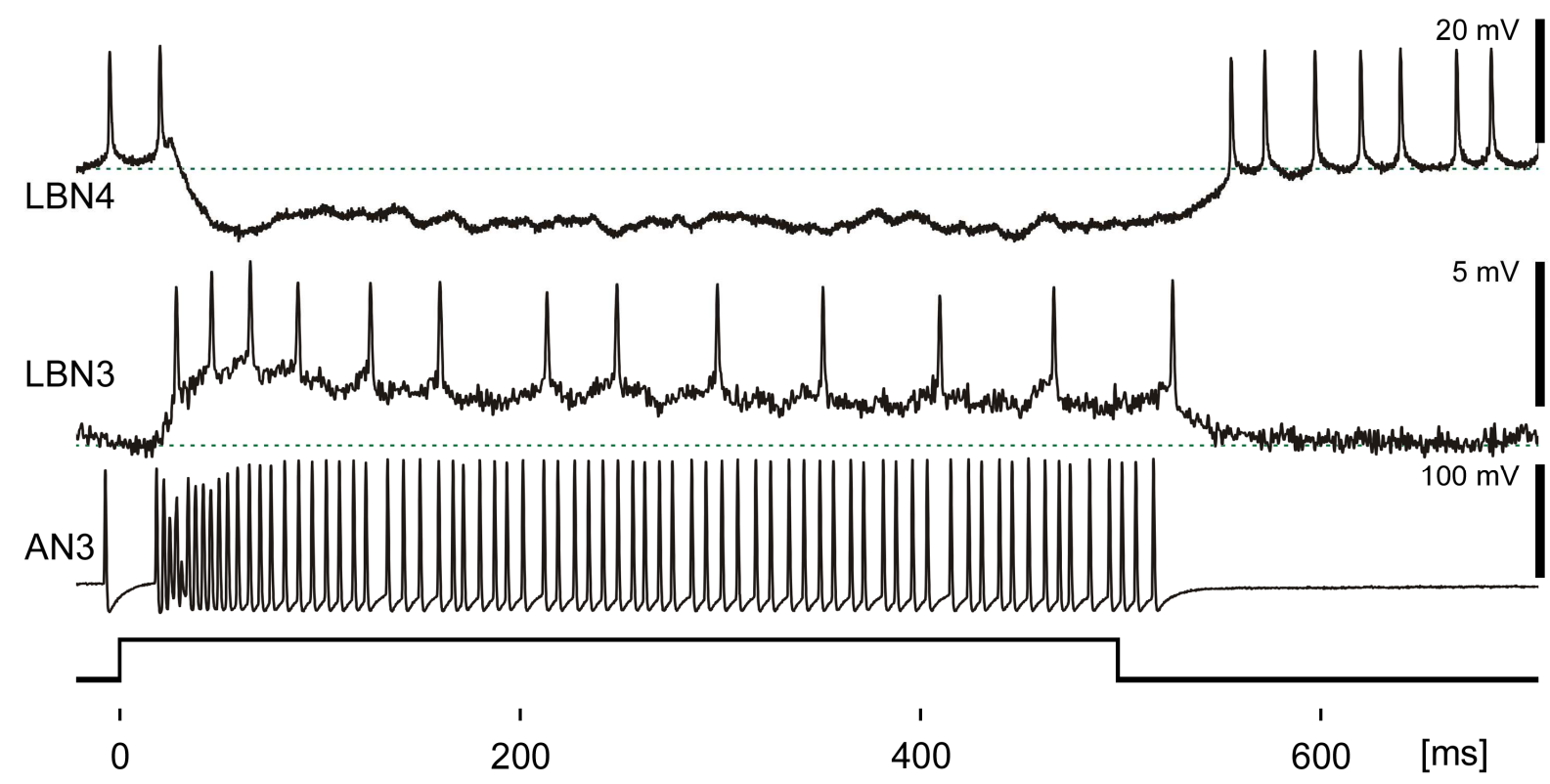

Figure 60: Example recordings of LBN4, LBN3 (filtered with a low-pass at $5000 \mathrm{~Hz}$ ) and AN3 to a 500 ms pulse (white noise, $70 \mathrm{~dB}$ SPL).

IPSP latencies of LBN4. The shortest IPSP latencies for LBN4 are found for $28 \mathrm{kHz}$ at $80 \mathrm{~dB}$ SPL with $26.8 \pm 2.6 \mathrm{~ms}$ ( $n=5$; mean and SD). This is a strong indication that LBN4 is not monosynaptically connected with an ascending neuron such as AN3 (shortest spike latencies are $19.2 \pm 0.4 \mathrm{~ms}$ - see chapter 3.1.3). A comparison to the shortest spike latency of LBN3 (26.8 $\pm 2.2 \mathrm{~ms}$; see chapter 3.2.3), on the other hand, shows a similar magnitude (both latencies obtained in different individuals). This finding suggests the involvement in the inhibitory pathway to LBN4 of (i) primary afferents that are responsible for the excitatory input to AN3, (ii) AN3 that causes the EPSP in LBN3 and (iii) possibly LBN3 that changes the excitatory information of AN3 to inhibition in LBN4 (Figure 61). 


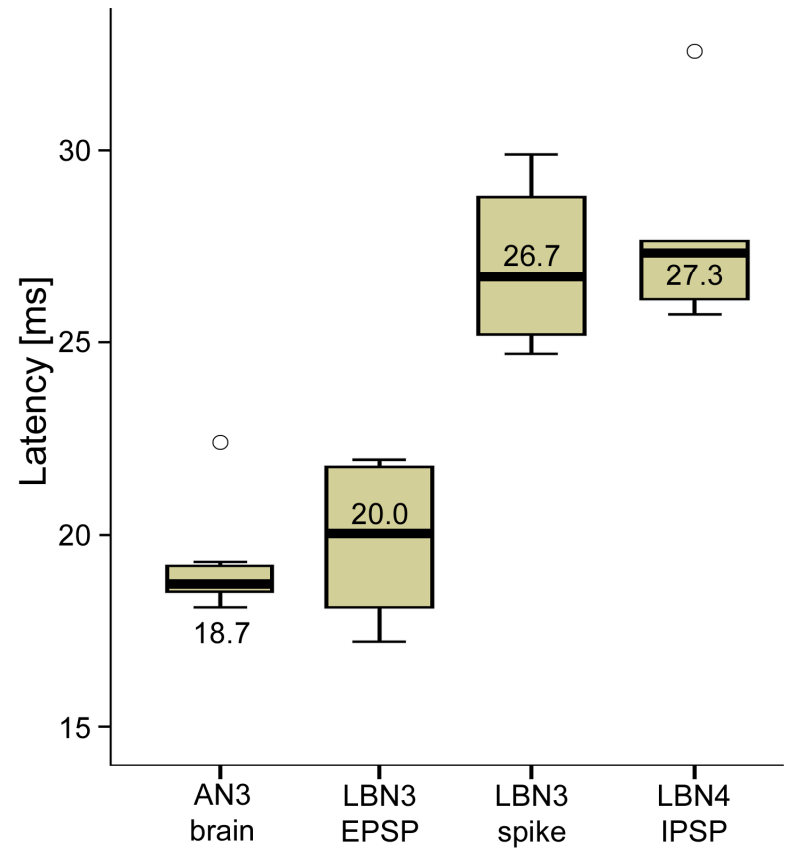

Figure 61: Box plot (see chapter 2.5) of the latencies of AN3 (brain recordings, $N=9$ ), LBN3 EPSP $(N=2, n=10)$, LBN3 spikes $(N=2, n=10)$ and LBN4 (IPSP, $\mathrm{n}=5$ ) for $28 \mathrm{kHz}$ at $70 \mathrm{~dB}$ SPL (the intensity with shortest spike latencies in AN3; 50 ms stimulus).

EPSP latencies of $L B N 4$ responses and its dependence on intensity. Besides the prominent IPSP in LBN4 also excitatory postsynaptic potentials preceding the IPSP can be found. In the beginning of the intracellular recording of LBN4 no spontaneous activity was seen, which allowed a clear identification of the EPSP latency (Figure 62B; no spontaneous activity was only seen for 8 and $16 \mathrm{kHz}$ of the standard stimuli program).

A

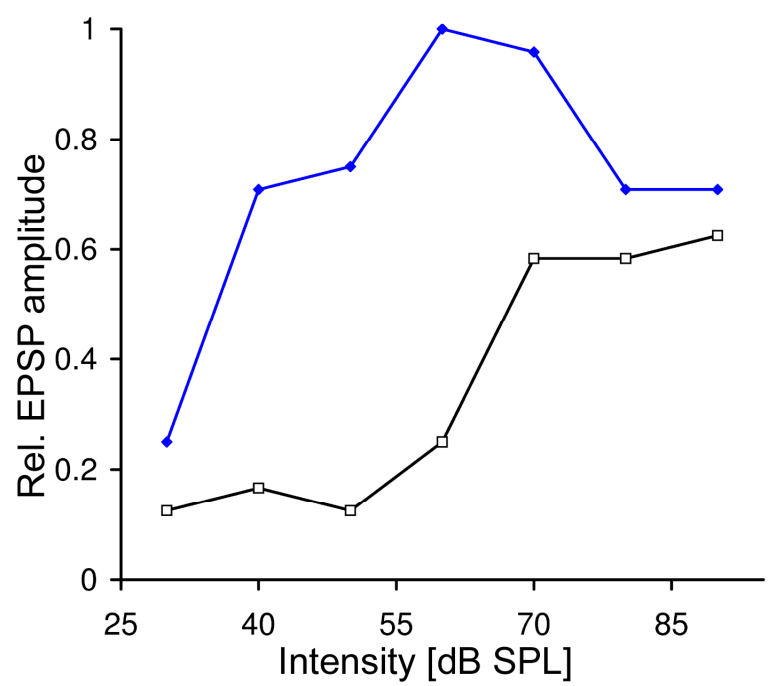

B response of LBN4

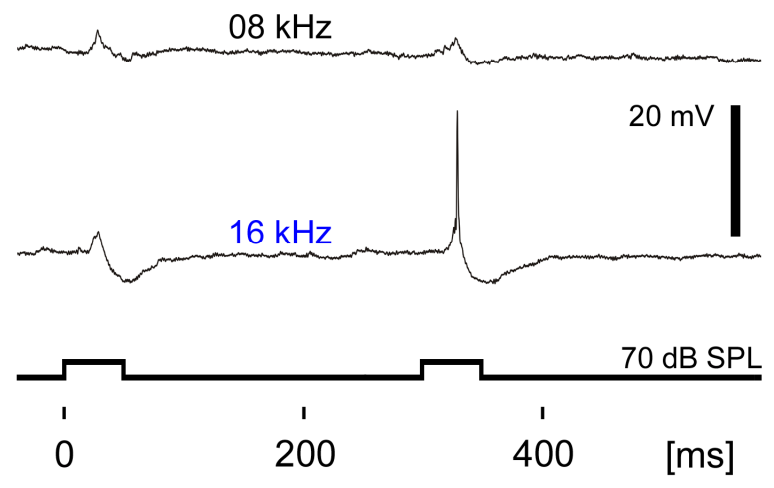

Figure 62: Response of LBN4 to standard stimuli. Blue colour - carrier frequency of the male song $(16 \mathrm{kHz})$. A: Relative amplitude of the EPSP in LBN4 calculated from the averaged responses ( $n=5$; means). B: Recording examples of LBN4 (filtered with a low-pass at $5000 \mathrm{~Hz}$ ) without spontaneous activity for 8 and $16 \mathrm{kHz}$ at $70 \mathrm{~dB}$ SPL. 
Shortest EPSP latencies were found for $16 \mathrm{kHz}$ at $50 \mathrm{~dB} \mathrm{SPL}$ with $19.3 \pm 0.5 \mathrm{~ms}$ $(n=5$; mean and SD). Such short latencies strongly suggest a direct connection with ascending interneurons. From the mean responses of LBN4 without spontaneous activity relative EPSP amplitudes could be measured (Figure 62A; only displayed for 8 and $16 \mathrm{kHz}$ ). For stimuli with $16 \mathrm{kHz}$ one can see optimum type characteristics with a low threshold. The threshold for $8 \mathrm{kHz}$ lies at approximately $60 \mathrm{~dB} S P L$ with a saturation of response at $70 \mathrm{~dB}$ SPL. The maximum EPSP amplitude, however, might be influenced by the beginning of the IPSP. From the response of LBN4 without spontaneous activity also the shortest spike latencies could be measured for $16 \mathrm{kHz}$ at $50 \mathrm{~dB}$ SPL with $25.3 \pm 1.9 \mathrm{~ms}(\mathrm{n}=5$; mean and SD).

\subsubsection{Crossing local brain neuron 5 (cLBN5)}

A neuron that can be easily recorded in axonal portions is cLBN5. Because of its morphology with an axon that crosses the midline of the brain (see below) the appendix "c" for "crossing" was added to the terminology of this neuron.

Morphology. A staining of CLBN5 was achieved once and the major branches are shown in Figure 63. The soma of cLBN5 is located directly underneath the posterior ventral surface of the protocerebrum.

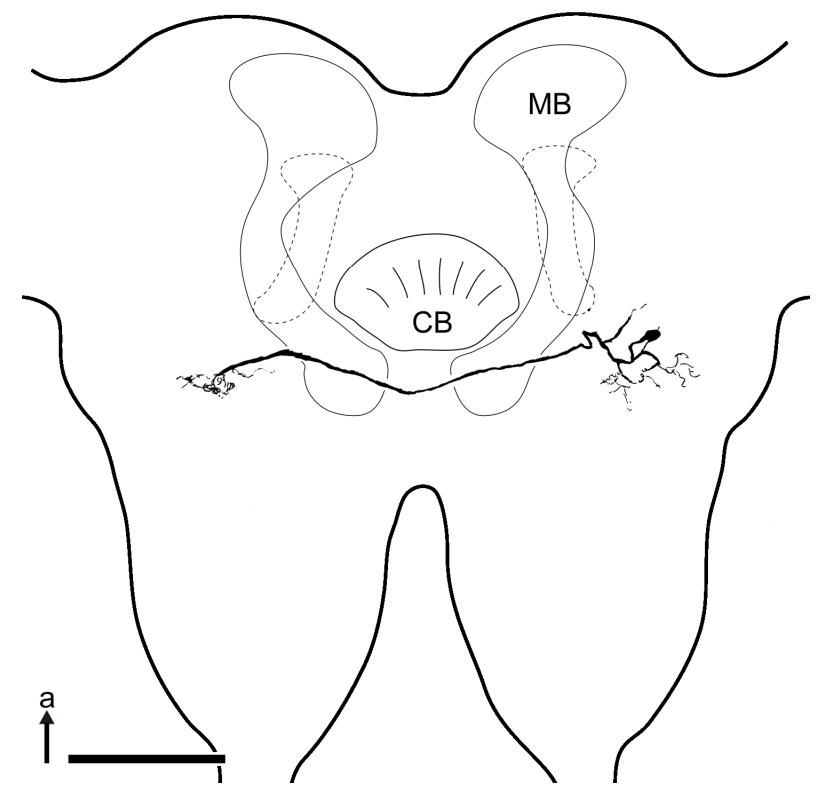

Figure 63: Morphology of an incomplete staining of CLBN5 in the brain of $A$. nigrovittata. Drawing after a Cy3-staining in relation to the central body (CB) and the mushroom bodies (MB). Bar represents $200 \mu \mathrm{m}$. a=anterior. 
The primary neurite runs dorsally and divides into two major branches in the horizontal section of the brain, where the central body can be seen to the greatest extent. One branch extends to lateral portions and arborises. The other branch - the main stem - extents medially and gives off 2 minor branches. The first minor branch runs posterior and arborises. The second branch splits off close to the pedunculus of the mushroom body and extends laterally. No arborisations could be detected at this branch, which was most likely due to an insufficient amount of staining substance in the neuron. The main stem runs to dorsal portions and then changes the direction towards the lateral protocerebrum of the contralateral side of the brain. In the medial dorsal portion of the contralateral protocerebrum only minor arborisations could be detected. These arborisations are most likely presynaptic since no graded potentials could be found in the respective intracellular recording to the staining.

Intensity and carrier frequency dependence of cLBN5 responses. Intracellular recordings were always obtained in axonal portions of cLBN5 and never in the dendritic region. The response to an artificial duet shows a burst of action potentials to the female answer only (Figure 64A). In two out of seven recordings occasionally few spikes were elicited to the male song. Responses to standard stimuli were weak (note the maximal spike numbers in Figure 64B). Slight responses can be observed to ultrasonic frequencies with lowest thresholds at $40 \mathrm{~dB}$ SPL. The threshold for 8 and $16 \mathrm{kHz}$ is $20 \mathrm{~dB}$ higher. Considerably higher spike numbers are found in cLBN5 when presenting the species specific duet with varying carrier frequency of the female reply (Figure 64C). The maximum activity can be found for ultrasonic frequencies (up to at least $50 \mathrm{kHz}$ ) with nearly no response at sonic frequencies. 
A
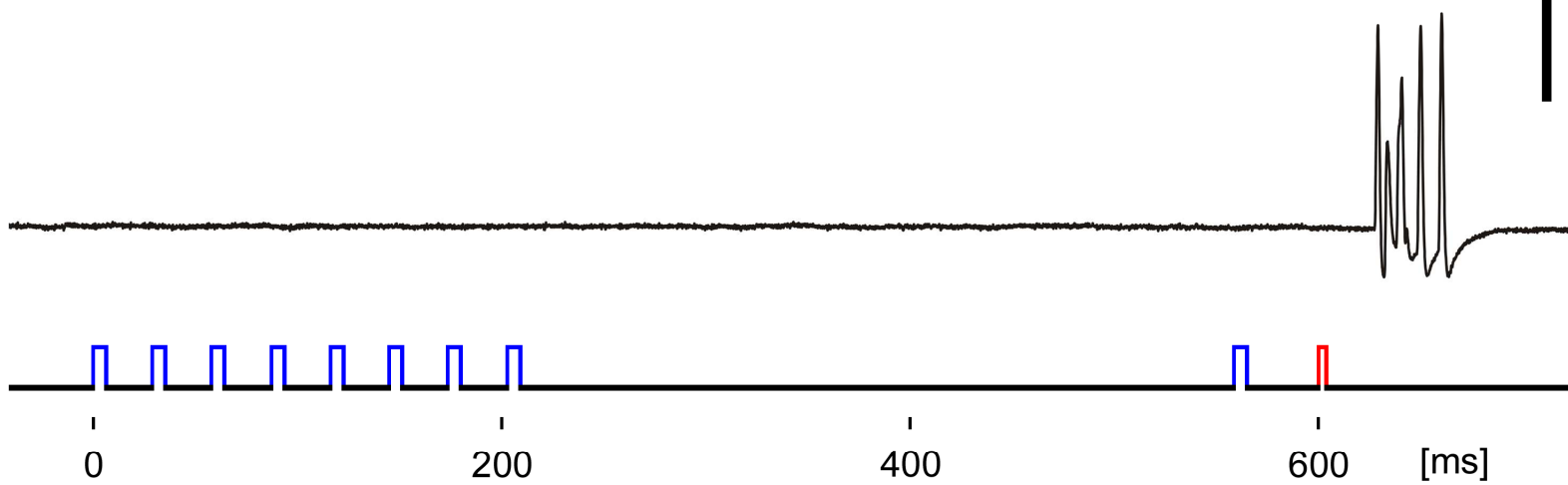

B $50 \mathrm{~ms}$ pulses

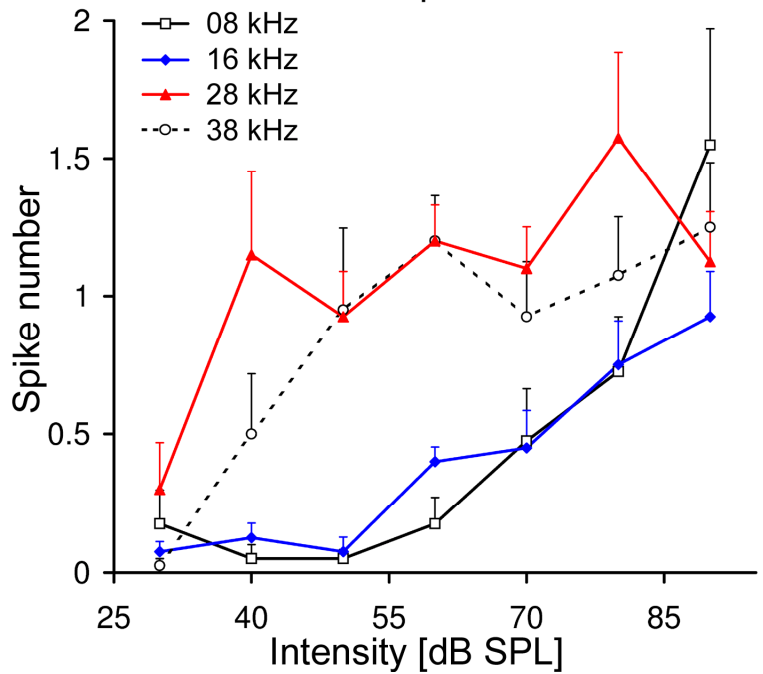

C frequency of the o-reply

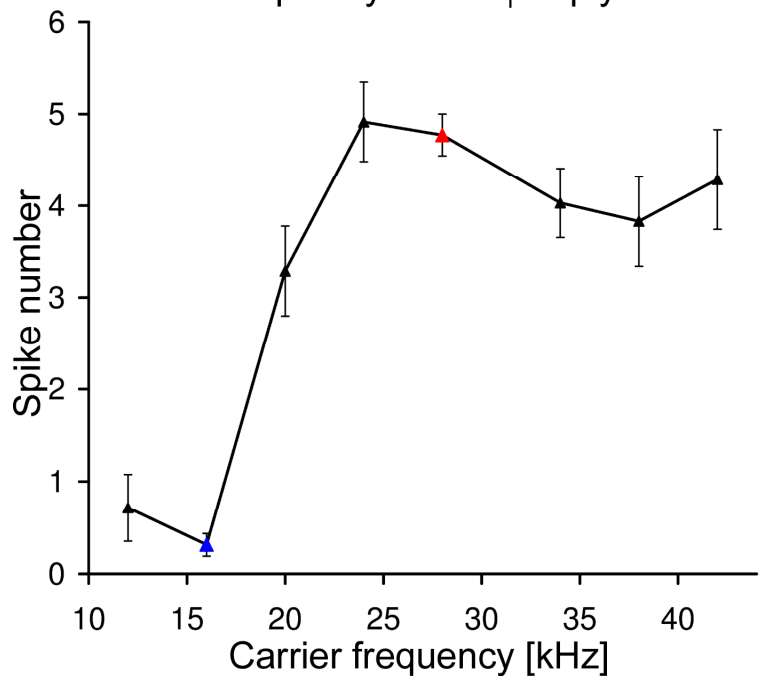

Figure 64: Response of cLBN5 to acoustic stimuli. Blue colouration - carrier frequency of the male song $(16 \mathrm{kHz})$ and red colouration - carrier of the female reply $(28 \mathrm{kHz})$. A: Example recording of the response to an artificial duet of $A$. nigrovittata (male song at $70 \mathrm{~dB}$ SPL; female answer at $60 \mathrm{~dB}$ SPL; see chapter 2.3.2 for stimulus paradigm). B: Response of CLBN5 to standard stimuli (see chapter 2.3.2) ( $\mathrm{N}=8$; means and $\mathrm{SE}$ ). C: Response to the female answer with varying carrier frequency in an artificial duet ( $\mathrm{N}=7$; see chapter 2.3.2 for stimulus paradigm; means and $\mathrm{SE})$.

Response of cLBN5 to varying temporal parameters. Acoustic stimulation of CLBN5 with white noise pulses at differing pause durations reveals in each individual the strongest spiking response at $15 \mathrm{~ms}$ pause duration with optimum type characteristic (Figure 65A). Short pause durations of 2 and $5 \mathrm{~ms}$ evoke nearly no responses whereby pauses at 35 and 61 ms show a $50 \%$ response. This decreased response to short pause durations parallels the female behaviour. The activity of cLBN5, however, most likely is not relevant for the recognition of male song models, since in an artificial duet its response only occurred to the female answer (in nearly all cases; see Figure 64A). Accordingly, stimulation with varying pulse durations reveals high spike numbers for the shortest pulses (Figure 65B left). Despite the high standard error at 2 ms pulse durations (that reflects a high intraindividual variability in 
total spike numbers), the strongest magnitude of response occurred in every individual always to the shortest pulse duration tested (Figure 65B right).

A

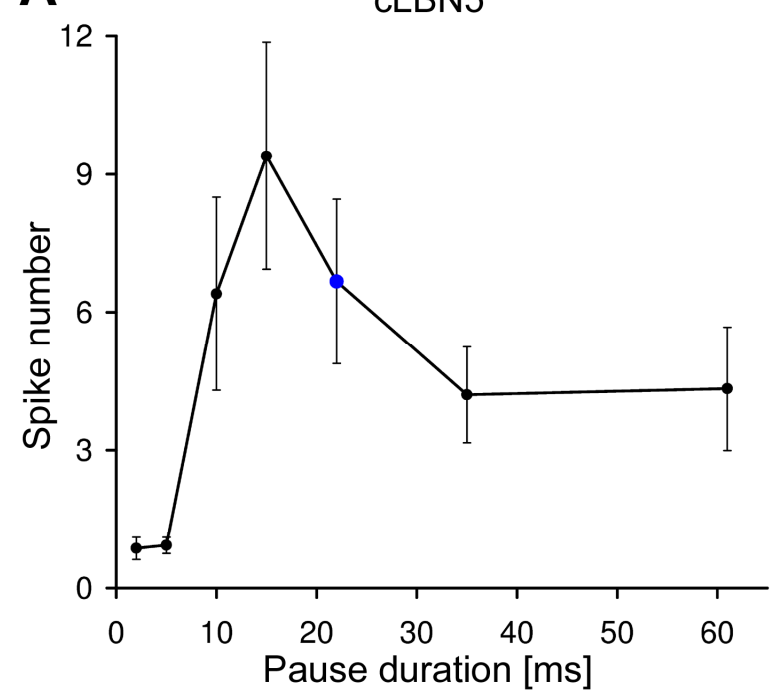

B

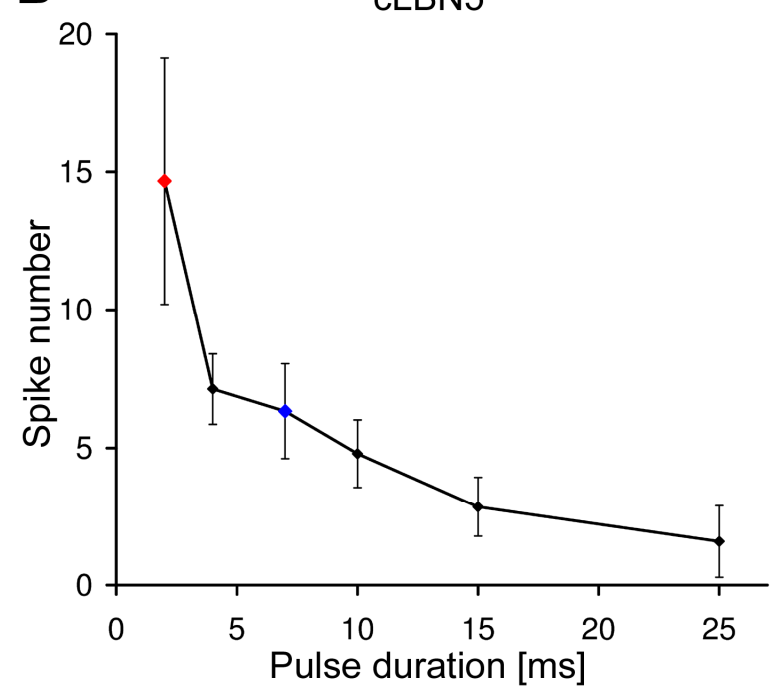

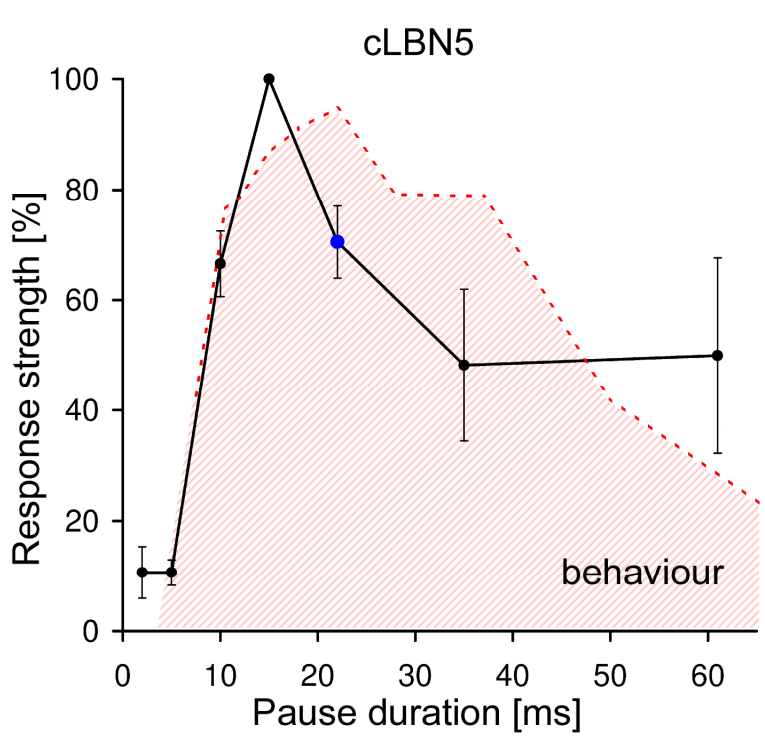

CLBN5

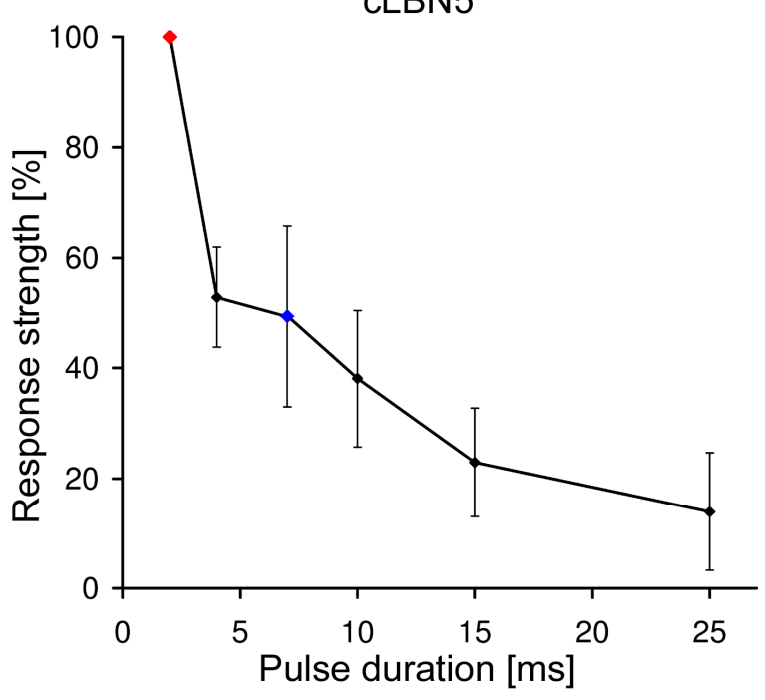

Figure 65: Mean response of cLBN5 to different temporal parameters (white noise; $70 \mathrm{~dB}$ SPL; see chapter 2.3.2 for stimulus paradigm; means and SE). Important natural parameters are emphasised (blue: male song pause/pulse duration; red: female reply pulse duration). A: Spike number (left) and magnitude of response (right) to stimuli with varying pause duration $(\mathrm{N}=3)$. Red shaded area indicates the female behaviour ( $N=5$, modified from Dobler et al. 1994a). B: Spike number (left) and magnitude of response (right) to stimuli with varying pulse duration $(\mathrm{N}=3)$.

Mirror-image partners of CLBN5. In one individual of A. nigrovittata it was possible to obtain intracellular recordings of both mirror-image partners of cLBN5 (identified by their physiology). Both cLBN5's were recorded in the axonal part, where no graded potentials could be observed. Similar overall frequency and intensity dependent characteristics were found for both mirror-image partners (Figure 66). Both partners exhibit low thresholds for ultrasonic frequencies and high thresholds for 
sonic frequencies. Also the overall spike number is similar in both neurons and may differ by maximal one spike at ultrasonic frequencies. This may mostly be due to the high variability of spike number within each cLBN5. The response of both cLBN5's to stimulation with an artificial duet and varying female answer carrier frequencies also look very similar (data not shown - the overall characteristics and spike number correspond to the mean response shown in Figure 64C).
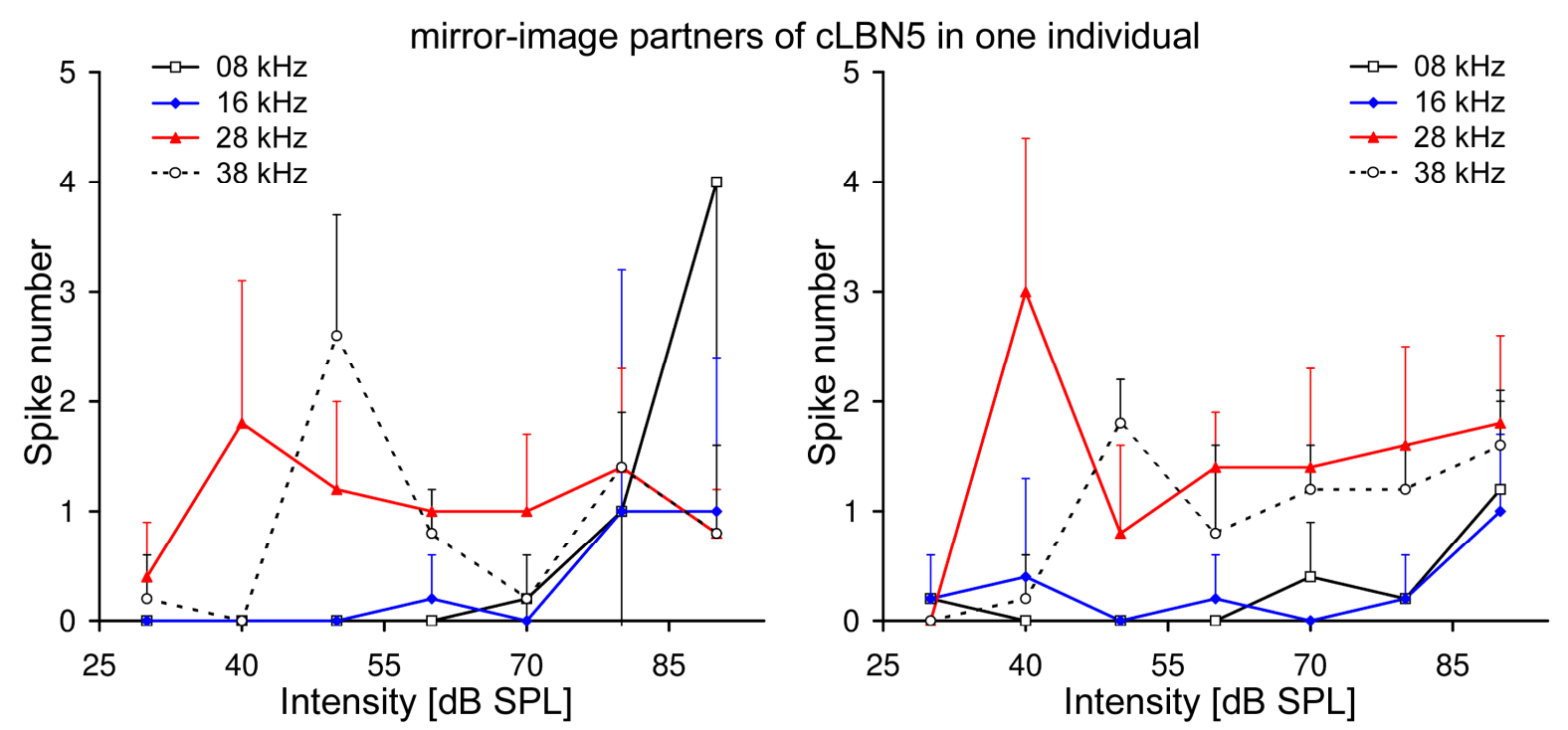

Figure 66: Response of both CLBN5 mirror-image partners in the same individual to standard stimuli (see chapter 2.3.2) ( $n=5$; means and SD). Blue colouration - carrier frequency of the male song $(16 \mathrm{kHz})$ and red colouration - carrier of the female reply $(28 \mathrm{kHz})$.

Spike latencies in CLBN5. The shortest spike latencies of CLBN5 for ultrasonic frequencies were found at $80 \mathrm{~dB}$ SPL with $35.3 \pm 2.7 \mathrm{~ms}(\mathrm{~N}=8$; mean and SE). This is similar to the spike latencies obtained for LBN2. Since no information is available about beginnings of graded potentials in cLBN5 no conclusions can be drawn concerning a possible connection to ascending neurons. However, the characteristics of cLBN5 do not correspond to those of any single ascending neuron. 


\subsubsection{Local brain neuron 6 (LBN6)}

LBN6 is a neuron that can be frequently recorded in the axonal portions in the lateral protocerebrum of the brain. It can be clearly identified by its response to low frequency stimuli. No staining could be obtained of LBN6 so far. Therefore, it is unknown whether LBN6 is an ascending neuron or a crossing local brain neuron. Intracellular recordings were obtained from 12 neurons but the amplitude of action potentials never exceeded approximately $10 \mathrm{mV}$. This may most likely be due to a small diameter of LBN6's axon and possibly indicate it being a local neuron.

Intensity and carrier frequency dependence of LBN6 responses. Only few LBN6s elicit occasionally one or two action potentials to a presentation of an artificial duet. But a response occurred always to a white noise 500 ms pulse (Figure 67A). A strong burst of spikes is elicited at the beginning of the pulse that reveals the phasic response characteristics of LBN6. Responses to standard stimuli show the strongest activity for $8 \mathrm{kHz}$ at $60 \mathrm{~dB} \mathrm{SPL}$ (Figure 67B). Also at $16 \mathrm{kHz}$ an optimum type characteristic is found with a much lower response, though. Ultrasonic frequencies elicit relatively weak responses at high intensities. Low thresholds and strong spiking activities to $8 \mathrm{kHz}$ were never observed for any ascending sound activated neuron in A. nigrovittata. Only vibratory neurons showed strongest activity at $8 \mathrm{kHz}$ but with a much higher threshold (see chapter 3.1.7). The responses of LBN6 to vibrational stimuli were weak and have been observed only occasionally. In Figure $67 \mathrm{C}$ the responses to ipsi- and contralateral stimulation (respectively to the side of penetration) at 8 and $16 \mathrm{kHz}$ of one LBN6 is displayed. The data obtained for both carrier frequencies are contrary. A lower response threshold and a higher maximum spike number are found for ipsilateral stimulation at $8 \mathrm{kHz}$. In contrast, the threshold at $16 \mathrm{kHz}$ is $10 \mathrm{~dB}$ lower for contralateral stimulation with similar maximum spike numbers. This finding suggests that LBN6 may receive excitatory input from both ears. 
A
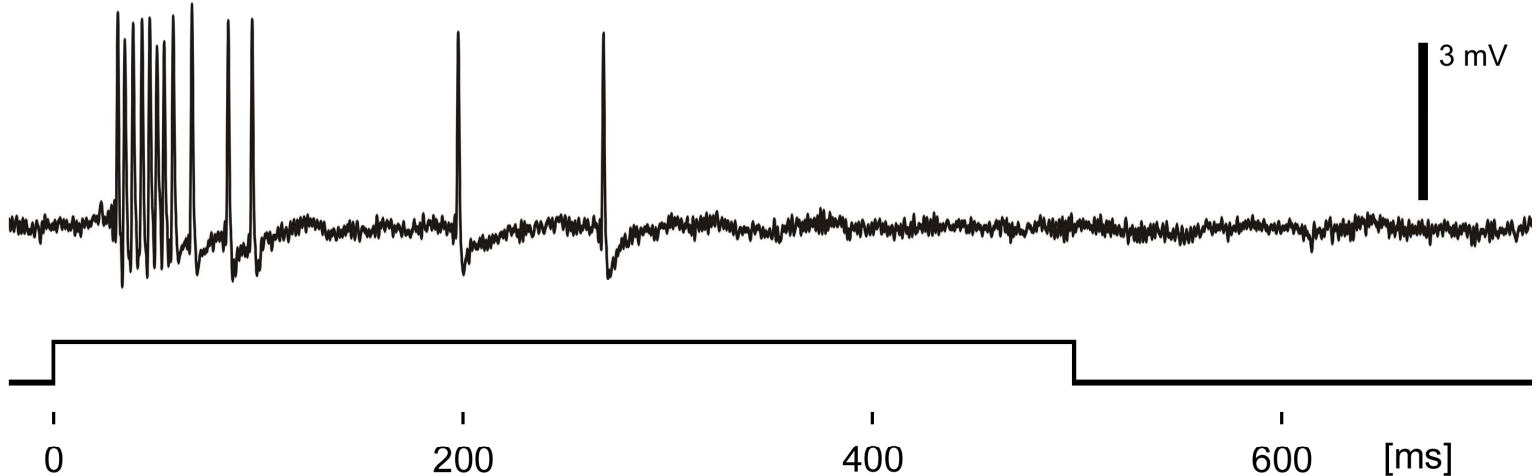

B

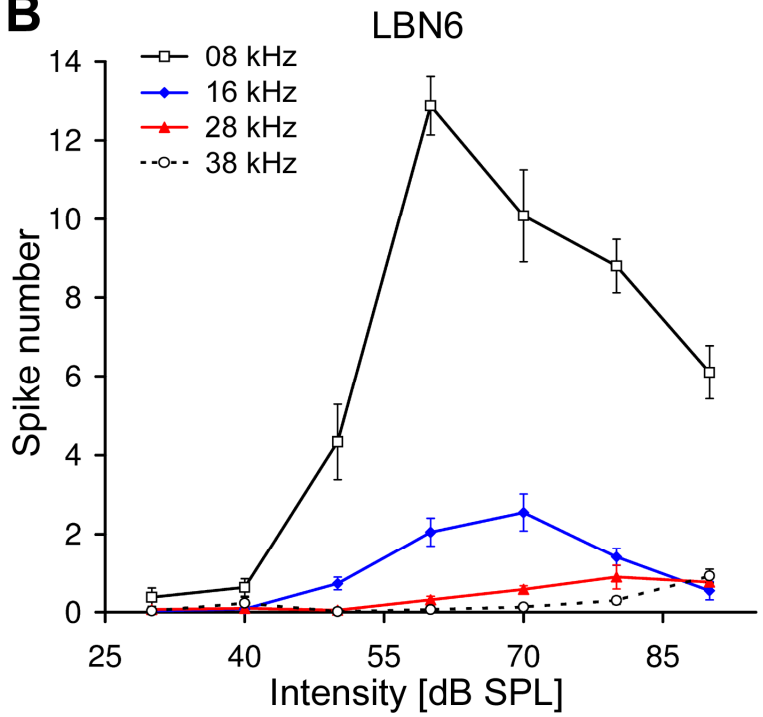

C ipsi vs. contra (for $08 \& 16 \mathrm{kHz}$ )

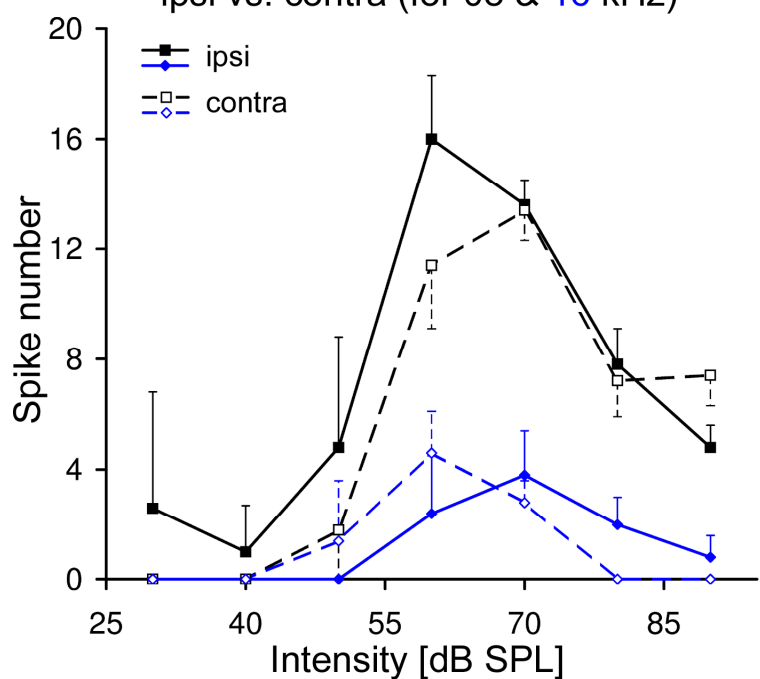

Figure 67: Responses of LBN6 to acoustic stimuli. Blue colouration - carrier frequency of the male song $(16 \mathrm{kHz})$ and red colouration - carrier of the female reply $(28 \mathrm{kHz})$. A: Example recording of the response to a $500 \mathrm{~ms}$ pulse (white noise, $70 \mathrm{~dB}$ SPL; filtered with a low-pass at $5000 \mathrm{~Hz}$ ). B: Response of LBN6 to standard stimuli (see chapter 2.3.2) ( $\mathrm{N}=12$; means and SE). C: Intensity dependent response of one LBN6 stimulated at 8 and $16 \mathrm{kHz}$ ipsi and contralateral of the recording site ( $n=5$; pulse duration: $50 \mathrm{~ms}$; means and SD).

Responses of $L B N 6$ to varying temporal parameters. The responses to differing pulse/pause durations of LBN6 are displayed in Figure 68. A clear response maximum can be seen for the shortest pause durations tested (2 ms; Figure 68A). For higher pause durations LBN6's response is about $50 \%$ reduced in comparison to the maximum. Pulse durations of $2 \mathrm{~ms}$ as well as pulse durations of $15 \mathrm{~ms}$ and longer reveal the strongest spiking activity (Figure 68B). The minimal response can be seen at 7 and $10 \mathrm{~ms}$. Taken the data of the varying temporal patterns together, one can see a reduced response of LBN6 to the natural male song pulse/pause durations (indicated by the blue data point in Figure 68). Also the specificity of the female behaviour to natural male song parameters exhibits a maximum where LBN6 has a response minimum. 
A

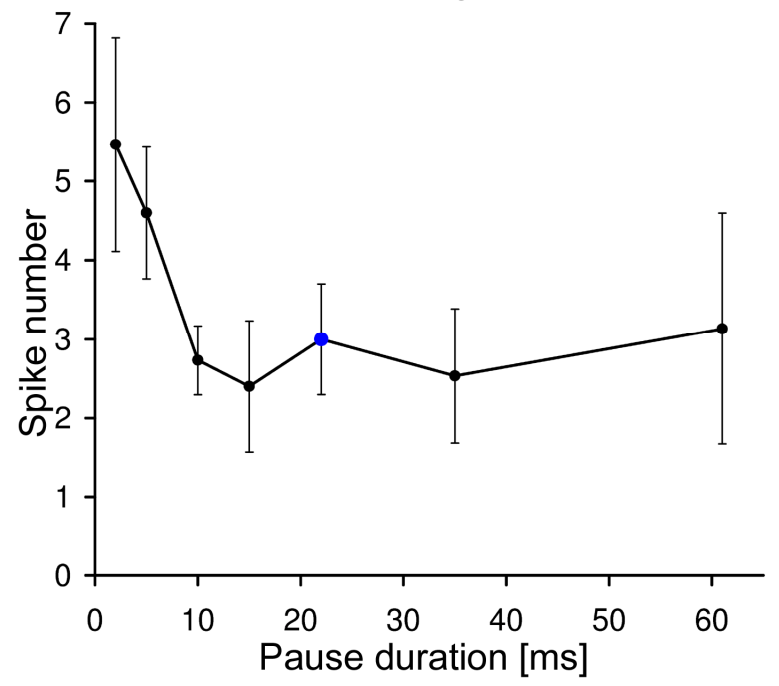

B

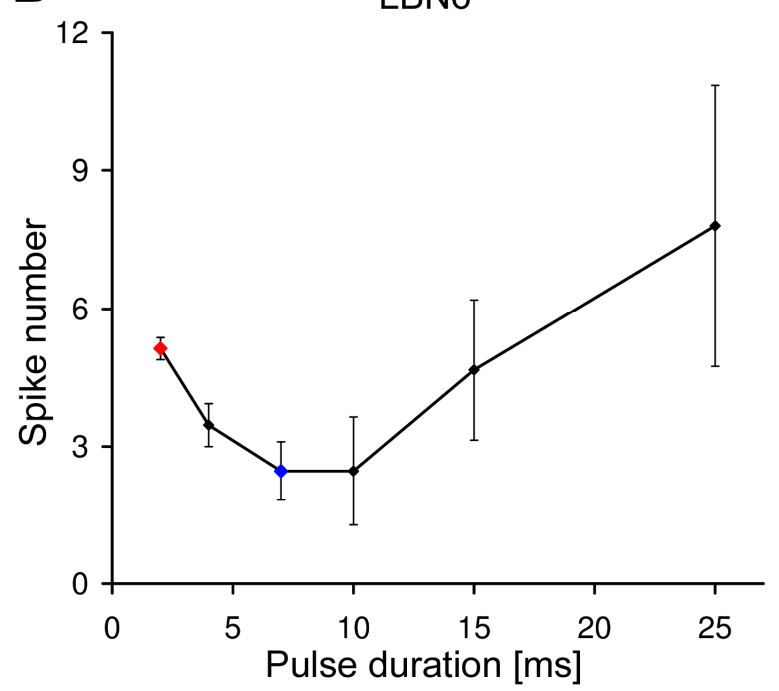

LBN6

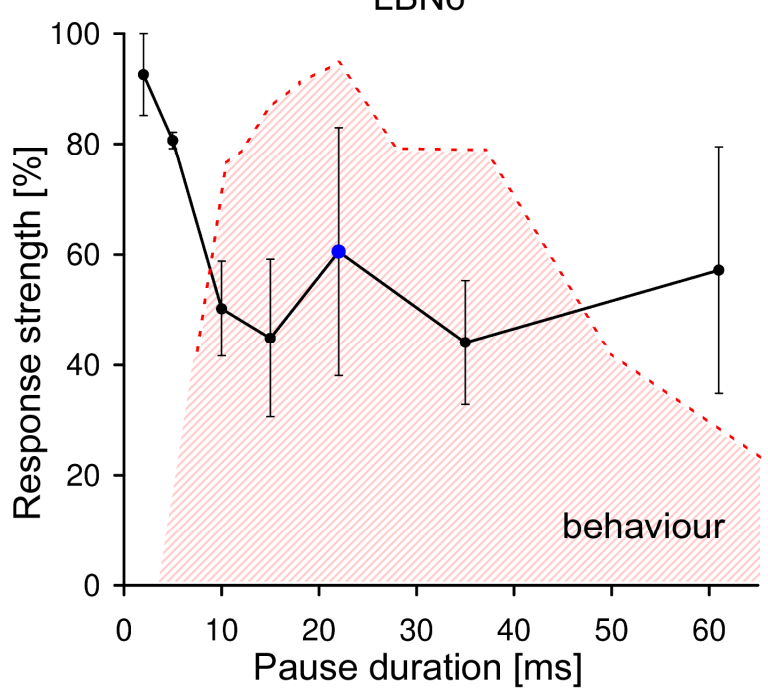

LBN6

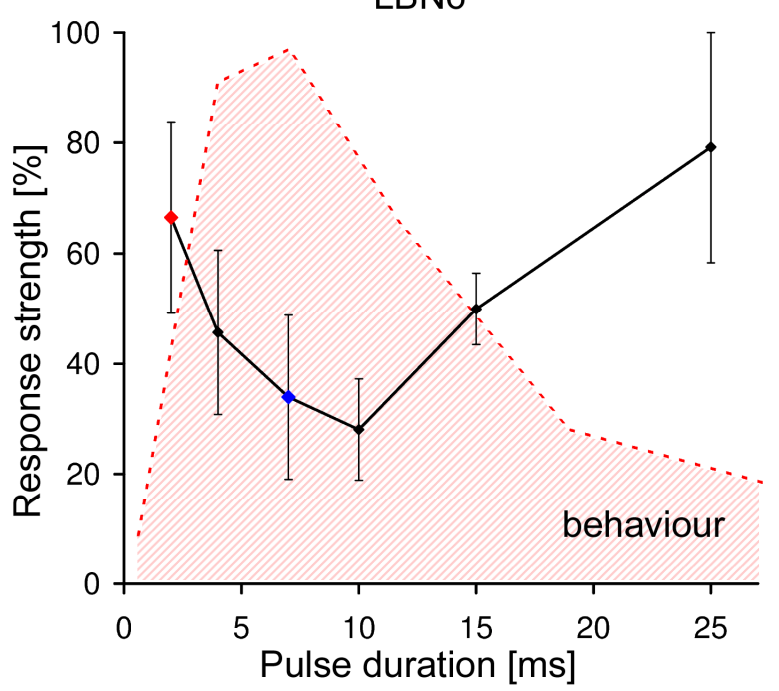

Figure 68: Mean responses of LBN6 to different temporal parameters (white noise; $70 \mathrm{~dB}$ SPL; see chapter 2.3.2 for stimulus paradigms; means and SE). Important natural parameters are emphasised (blue: male song pause/pulse duration; red: female reply pulse duration). Red shaded area indicates female behaviour ( $N=5$, modified from Dobler et al. 1994a). A: Spike number (left) and magnitude of response (right) to stimuli with varying pause duration $(\mathrm{N}=3)$. B: Spike number (left) and magnitude of response (right) to stimuli with varying pulse duration $(\mathrm{N}=3)$.

Spike latencies in LBN6. Shortest spike latencies with $34.7 \pm 1.0 \mathrm{~ms} \quad(\mathrm{~N}=12$; mean and SE) are found at $8 \mathrm{kHz} 60 \mathrm{~dB}$ SPL. This result is strong evidence against the possibility of LBN6 being an ascending interneuron. For comparison, shortest spike latencies for sound activated ascending neurons were between 18.6 ms (AN1) and $24.9 \mathrm{~ms}(\mathrm{ANx})$. 


\subsubsection{Local brain neuron 7 (LBN7)}

LBN7 is a neuron that is rarely recorded in the brain. In the following, the morphology and physiology of one LBN7 will be exemplarily presented. This data was confirmed by a further staining and recording in a different specimen.

Morphology. The spatial extent of LBN7 is restricted to one side of the brain (Figure 69A). Its soma is situated in the dorsal medial portion of the hemiganglion (in Figure 69B, C indicated by an arrow). The primary neurite runs ventrally and splits into one minor and two major branches. The minor branch extends to anterior ventral regions and arborises densely. One of the major branches runs to lateral ventral portions and two to three collaterals split off that arborise in posterior and dorsal regions of the brain. The main stem of the major branch, however, takes course to more ventral anterior portions and arborises densely in the same area where the minor branch also exhibits its ramifications. The dense arborisations seem to have beaded ending indicating to be presynaptic.

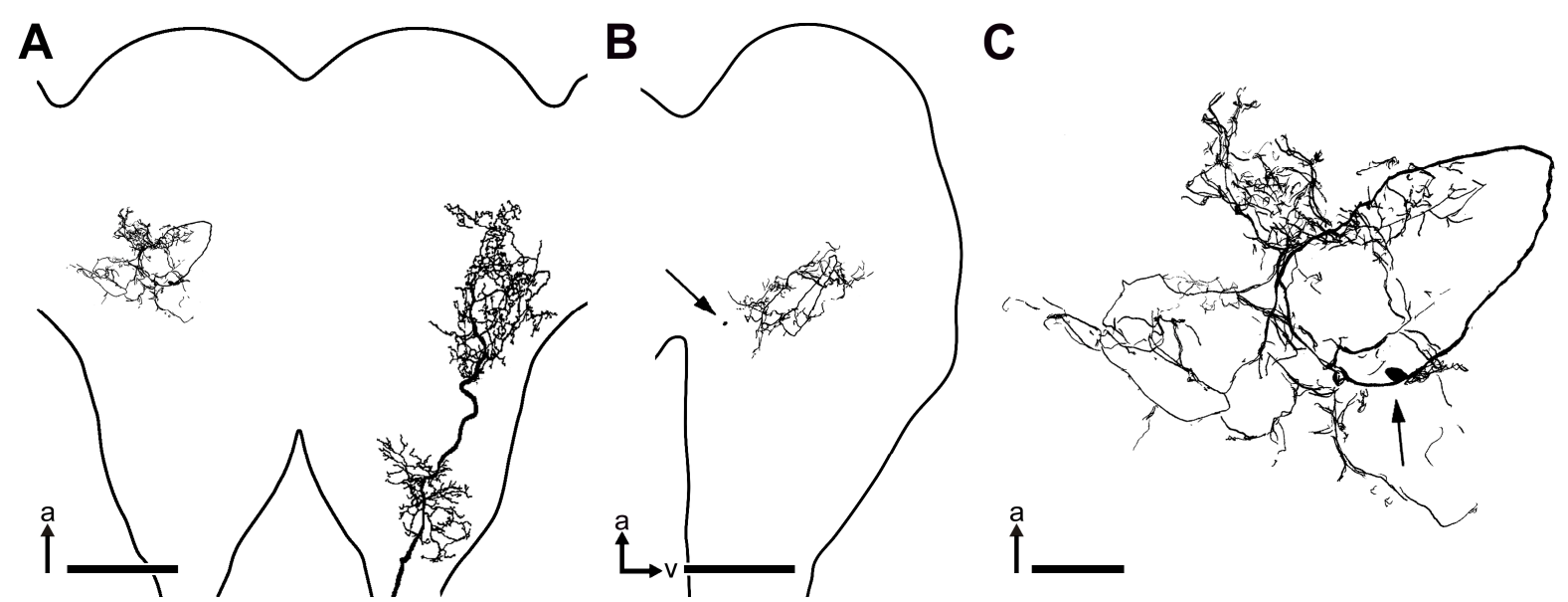

Figure 69: Drawing of LBN7 and AN2 in the brain of A. nigrovittata. A: Horizontal overview of LBN7 left and AN2 right (taken von Stumpner \& Molina 2006). Bar represents $200 \mu \mathrm{m}$. B: Sagittal overview in the brain. The arrow marks the soma of LBN7. Bar represents $200 \mu \mathrm{m}$. C: Detailed view on LBN7.

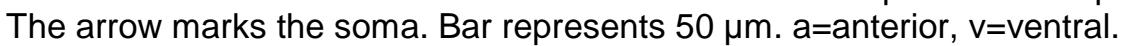

The other major branch extends to medial ventral portions without arborising. Then, it changes the direction and runs to lateral portions of the brain. Here, arborisations are found that have smooth endings indicating to be postsynaptic. The stem of this major branch now extends to more dorsal portions and a few arborisations are found that appear to have beaded endings. In Figure 69A also the arborisations of AN2 from a different individual are displayed (LBN7 seems to be monosynaptically connected to 
AN2 - see below). For alignment of the two neurons the position of midline, deutoand protocerebrum was taken as a reference. The possible site of connection could not be detected since AN2's arborisations widely overlap with these of LBN7.

Intensity and carrier frequency dependence of LBN7 responses. In Figure 70A an example recording of LBN7 to an artificial duet is shown. A response can be seen at the beginning of the male calling song as well as to the male trigger pulse and the female reply. The strongest spiking activity, though, can be observed to the female reply.

A

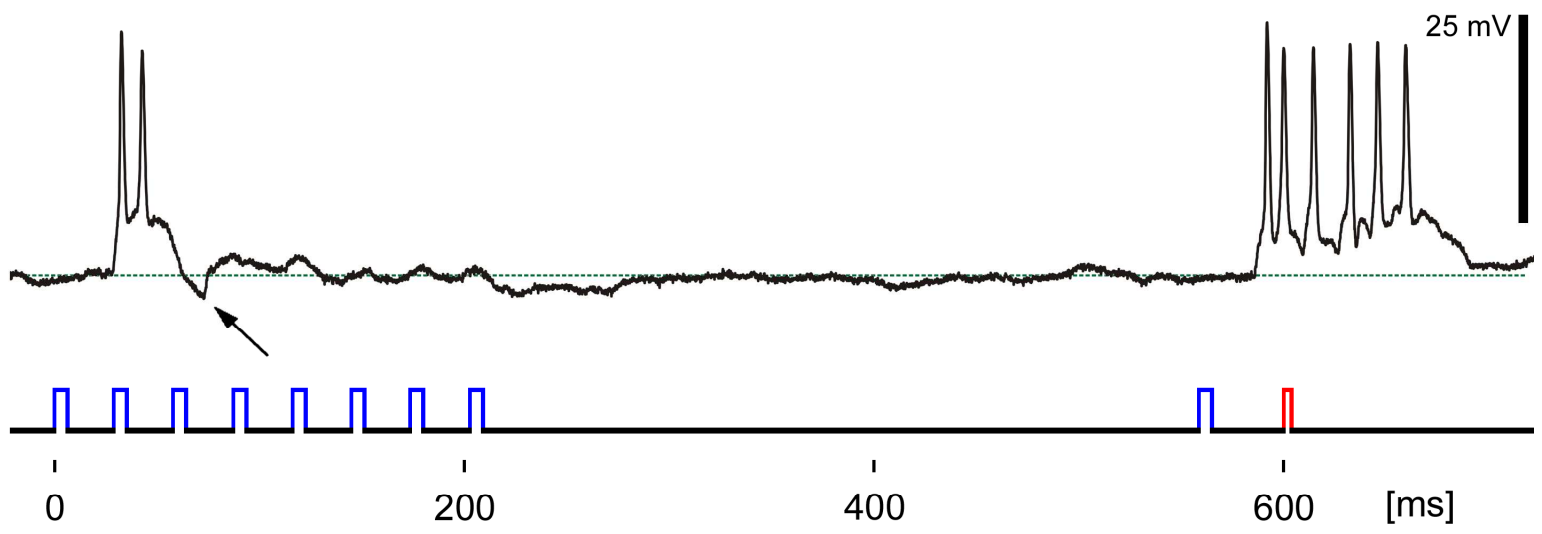

B

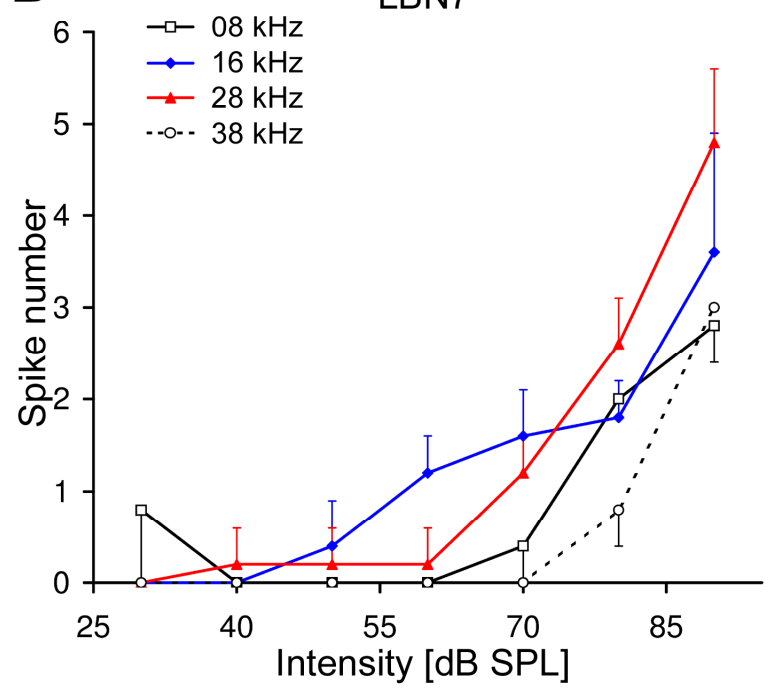

C

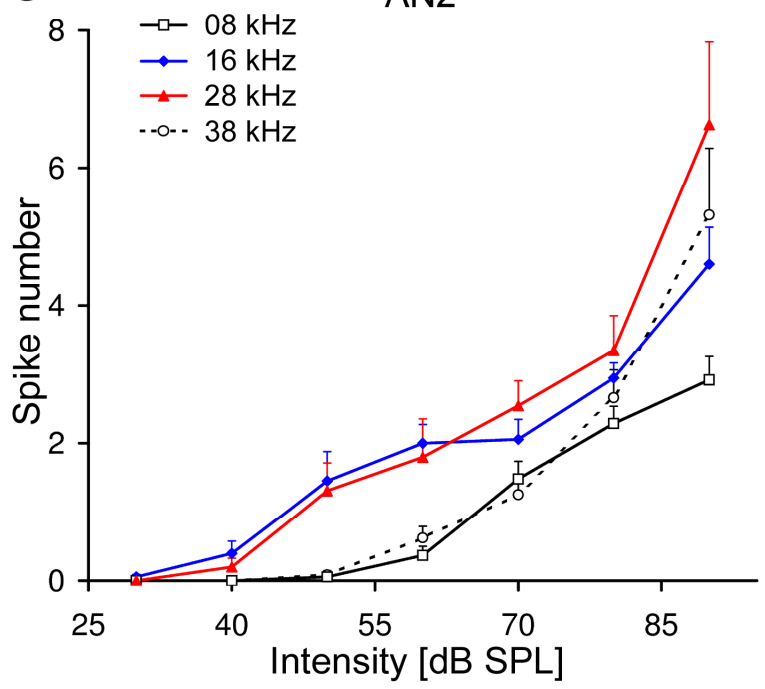

Figure 70: Response to acoustic stimuli. Blue colouration - carrier frequency of the male song $(16 \mathrm{kHz})$ and red colouration - carrier of the female reply $(28 \mathrm{kHz})$. A: Example recording of the response of LBN7 (depolarised) to an artificial duet of $A$. nigrovittata (male song at $70 \mathrm{~dB}$ SPL; female answer at $60 \mathrm{~dB}$ SPL; see chapter 2.3.2 for stimulus paradigm). The arrow marks the IPSP. B: Response of LBN7 (depolarised) to standard stimuli (see chapter 2.3.2) ( $n=5$; means and SD). C: Response of AN2 to standard stimuli (see chapter 2.3.2) ( $\mathrm{N}=7$; means and $\mathrm{SE}$ ). 
Obviously, the excitatory input to the male pulse series in LBN7 gets inhibited (arrow in Figure 70A) by a strong IPSP (subsequent to excitation). Thus, only few spikes are elicited at the beginning. LBN7's response to $50 \mathrm{~ms}$ pulses at increasing intensity and varying carrier frequency shows lowest thresholds for $16 \mathrm{kHz}$ stimuli (50 dB SPL; Figure 70B). The response threshold to ultrasonic frequencies can be seen for $28 \mathrm{kHz}$ at $70 \mathrm{~dB}$ SPL and for $38 \mathrm{kHz}$ at $80 \mathrm{~dB}$ SPL. The overall characteristic of the spiking activity of LBN7 to standard stimuli is very similar to the spiking activity of AN2 (Figure 70C) although a difference is found in the responses to $28 \mathrm{kHz}$ at 50 and $60 \mathrm{~dB} \mathrm{SPL}$ (this difference is not seen in the responses of AN2 when recorded in the thorax - compare to Figure 10B). A comparison of LBN7's response to ipsi- and contralateral stimulation (relative to the side of penetration) is displayed in Figure 71 (see also Figure 76). At stimuli with $28 \mathrm{kHz}$ the ipsi-/contralateral difference is much larger than at $16 \mathrm{kHz}$. This indicates that the inhibition found in LBN7 originates (at least partially) from the contralateral ear and may contribute to the reduced spiking at medium intensities at $28 \mathrm{kHz}$ when compared to the responses of AN2 (Figure 70C).

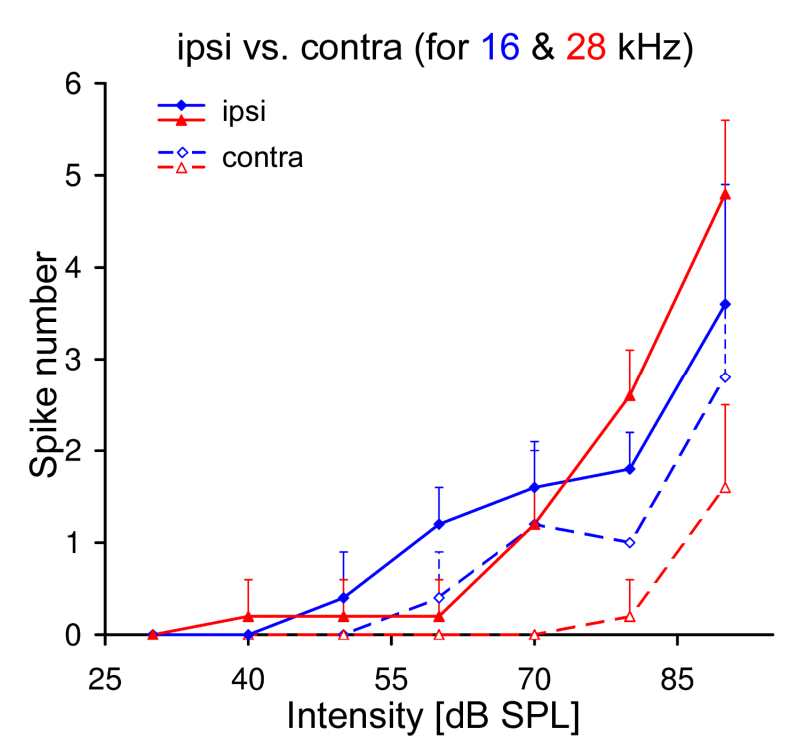

Figure 71: Intensity dependent response of one LBN7 stimulated at 16 and $28 \mathrm{kHz}$ ipsi- and contralateral of the recording site $(n=5$; pulse duration: $50 \mathrm{~ms}$; means and SD).

Responses of LBN7 to varying temporal parameters. The response of LBN7 to variations in pause duration is displayed in Figure 72A. Highest spike numbers are found with pause durations of 35 and $61 \mathrm{~ms}$. The strong decrement of response with shorter pauses may be due to the delayed IPSP that inhibits the excitation and prevents LBN7 from spiking when pauses are shorter than $35 \mathrm{~ms}$ (Figure 72B). Only a burst of a few action potentials can be seen at the beginning of the pulse series. The strong dependence of LBN7's spiking on pause duration may therefore be due to the duration of the IPSP that ends before the next pulse occurs (only at pause 
durations of $35 \mathrm{~ms}$ or longer). A similar effect can also be observed in AN2 (see Figure 12A).
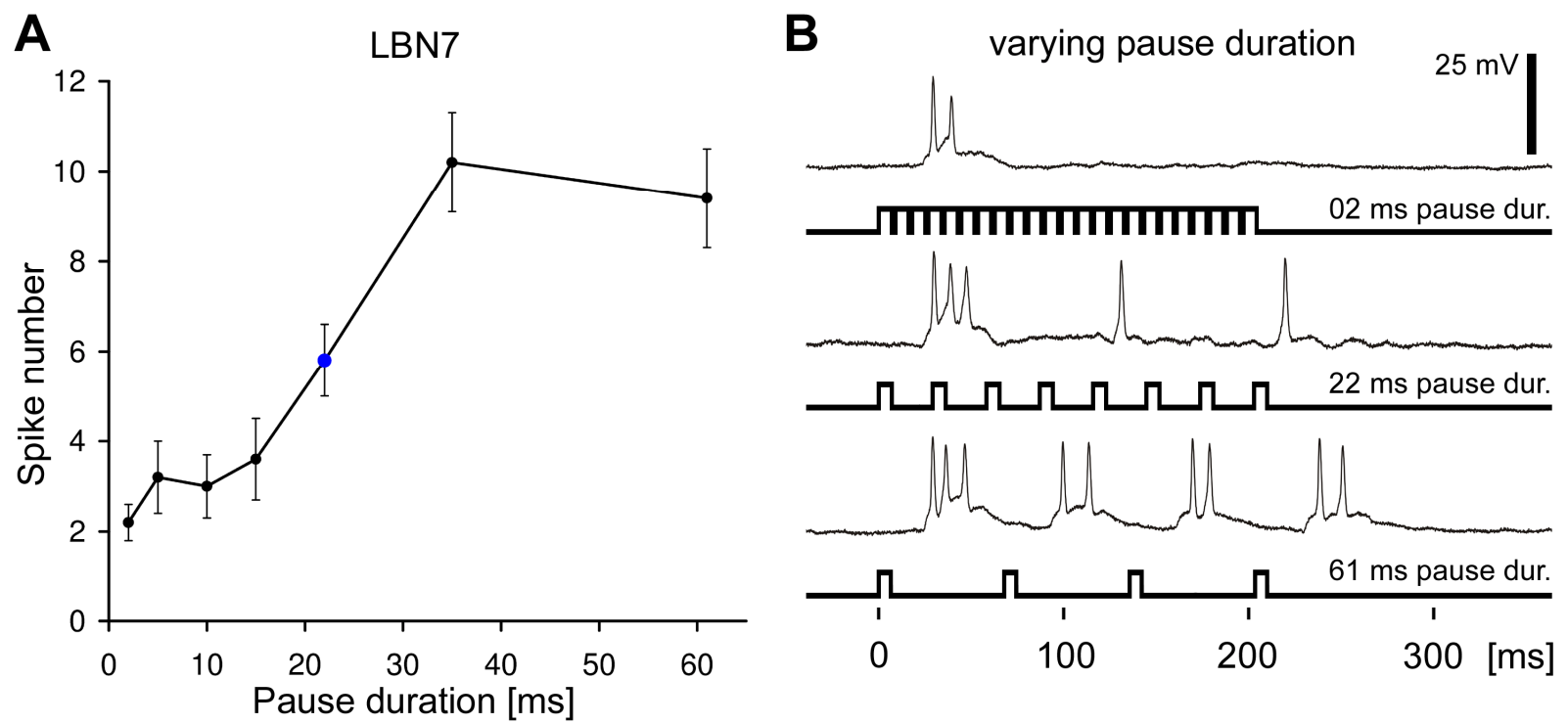

Figure 72: Response of LBN7 (depolarised) to different pause durations (white noise; $70 \mathrm{~dB}$ SPL; see chapter 2.3.2 for stimulus paradigms). A: Spike number to stimuli with varying pause duration $(n=5$; means and SD). Important natural parameters are emphasised (blue: male song pause duration). B: Example recordings to stimulation with pulses divided by 2, 22 and $61 \mathrm{~ms}$ pauses.

The response of LBN7 to a presentation of stimuli with varying pulse duration is displayed in Figure 73.
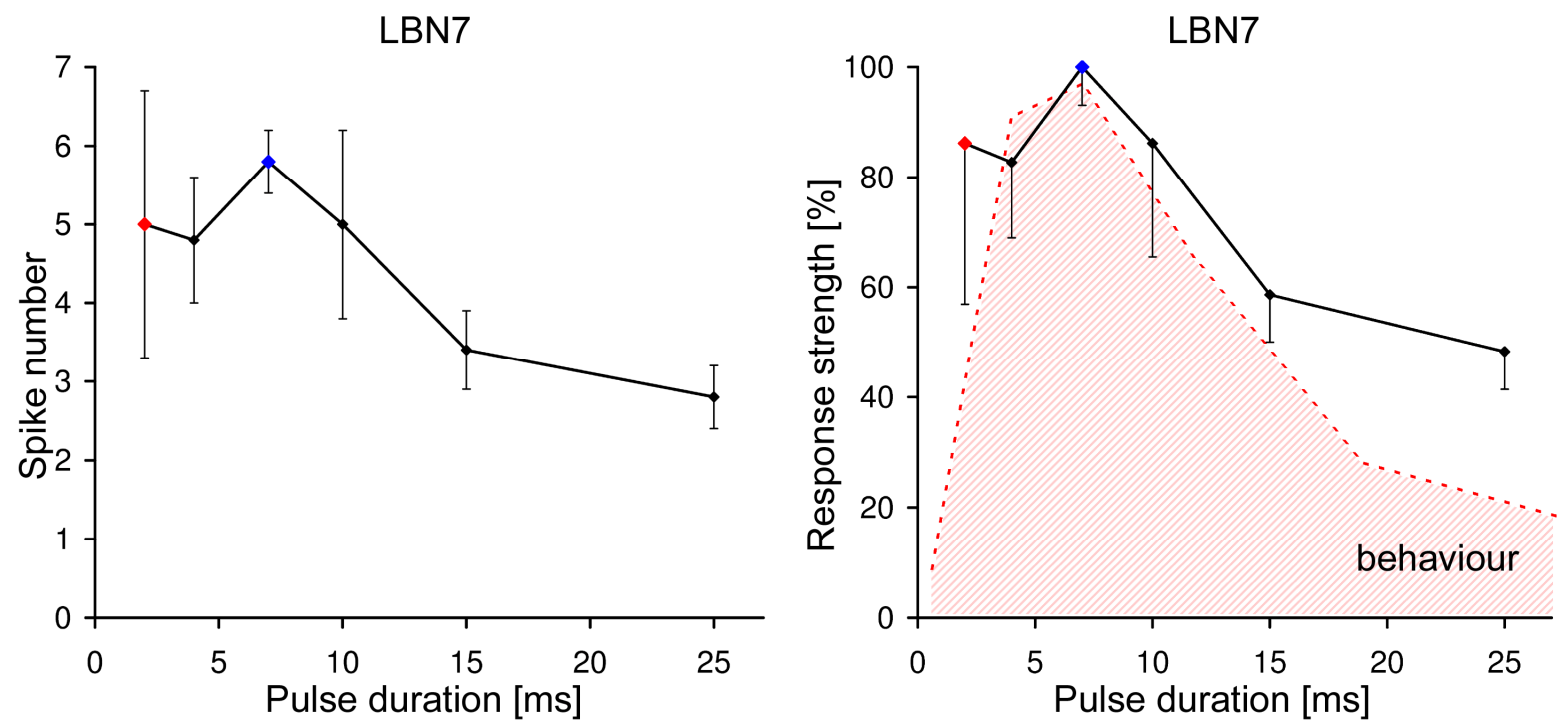

Figure 73: Response of LBN7 (depolarised) to different pulse durations (white noise; $70 \mathrm{~dB}$ SPL; see chapter 2.3.2 for stimulus paradigms; $n=5$; means and SD). Spike number is displayed left and magnitude of response right. Important natural parameters are emphasised (blue: male song pulse duration; red: female reply pulse duration). Red shaded area indicates female behaviour $(\mathrm{N}=5$, modified from Dobler et al. 1994a). 
The highest spike numbers are revealed for stimuli with short pulse durations (shorter or equally to $10 \mathrm{~ms}$ ). The maximum response is comparatively smaller than the maximum for variations in pause duration. This is most likely due to the suboptimal pause duration of the varying pulse paradigm (pause duration: $22 \mathrm{~ms}$; see response maximum in Figure 72A). In comparison to the behaviour, the relative spike numbers of LBN7 closely correspond with the decreasing response of female A. nigrovittata to longer pulse durations than $7 \mathrm{~ms}$. Such a close correlation of relative spike number and female behaviour at increasing pulse duration can also be observed in AN2 (see Figure 12B).

Response to different frequencies of the female answer in an artificial duet. When stimulating with an artificial duet and variation in carrier frequency of the female reply a strong response of LBN7 can only be seen at 24 and $28 \mathrm{kHz}$ with optimum type characteristic (Figure 74A; similar characteristics are found in AN2 see Figure 11). Stimuli with higher or lower carrier frequencies only elicit very few action potentials. To $50 \mathrm{~ms}$ pulses nearly no responses in LBN7 can be seen for $28 \mathrm{kHz}$ at $60 \mathrm{~dB} \mathrm{SPL}$ (the female reply in the artificial duet is also presented at $60 \mathrm{~dB}$ SPL; see also Figure 70B).

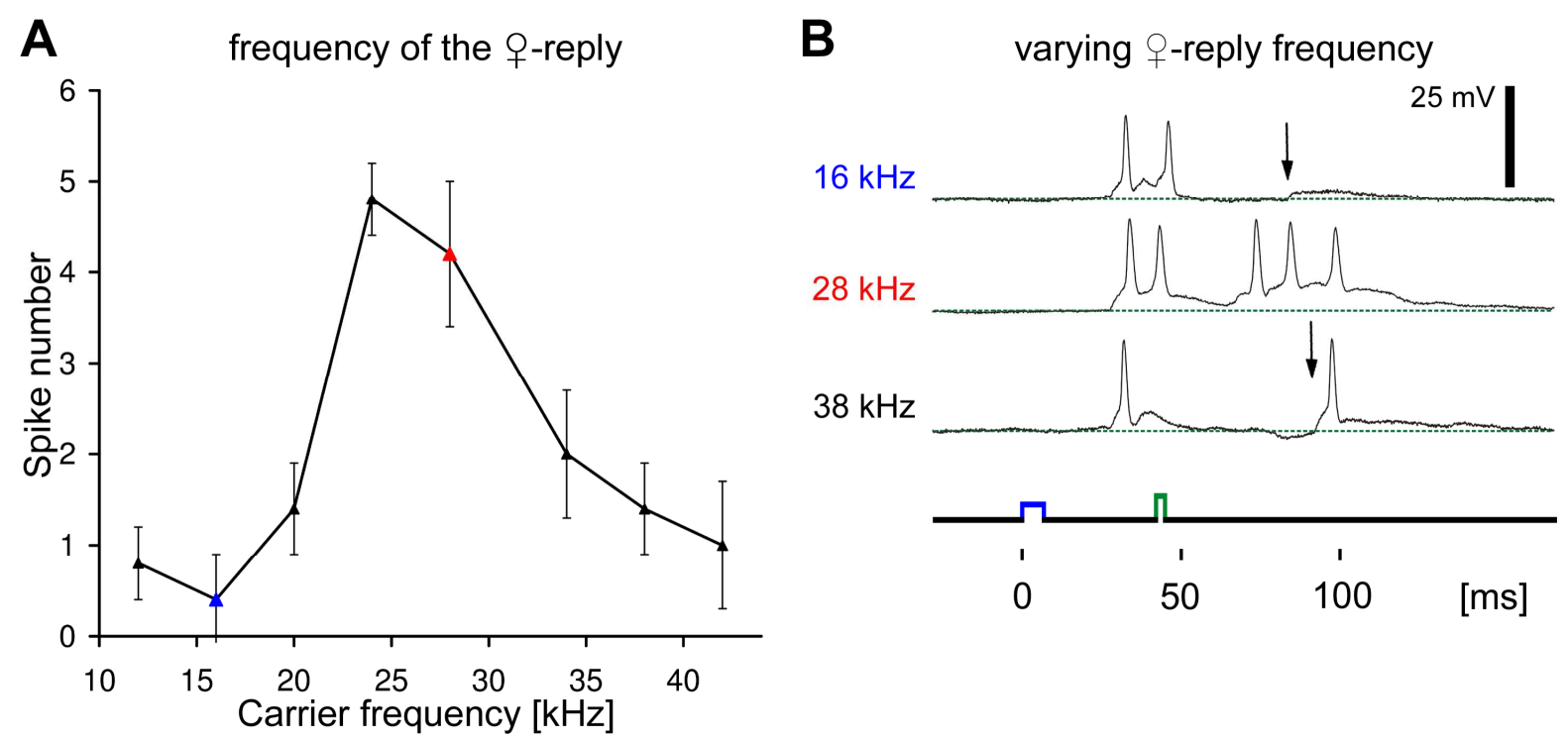

Figure 74: Response of LBN7 (depolarised) to the female answer with varying carrier frequency in an artificial duet (see chapter 2.3.2 for stimulus paradigm). Blue indicates the male's natural carrier frequency and red the natural frequency of the female reply. A: Spike numbers of LBN7 ( $n=5$; means and SD). B: Recording examples of LBN7's response to the female reply at 16,28 and $38 \mathrm{kHz}$. The arrows mark the beginnings of excitation to stimuli with frequencies outside the best range of response. 
In the example recording (shown in Figure 74B) one can clearly see the strong response to $28 \mathrm{kHz}$. The slight response to stimuli with 16 or $38 \mathrm{kHz}$ is shifted to higher latencies (see arrows in Figure 74B). This might be due to a delayed inhibition caused by the male trigger pulse, although direct evidence has not been observed. Stimulation with carrier frequencies outside the best response of LBN7 might only cause a small excitatory input to LBN7 that may be affected by the delayed IPSP. Additionally, LBN7 seems to receive inhibition in the higher ultrasonic frequency range (note the initial IPSP to the female reply at $38 \mathrm{kHz}$ ).

However, at this stimulus paradigm the female reply has a constant latency of $35 \mathrm{~ms}$. This also is the pause duration where strongest response in LBN7 can be observed in the paradigm with the varying pause durations in a model of the male song with white noise (see Figure 72). To test whether the IPSP may influence the response to short $28 \mathrm{kHz}$ stimuli, the latency of the female answer pulse in an artificial duet was varied (Figure 75). Independent of the latency of the female reply to the trigger pulse, $28 \mathrm{kHz}$ elicits a strong excitatory response in LBN7, despite a potential delayed inhibition.

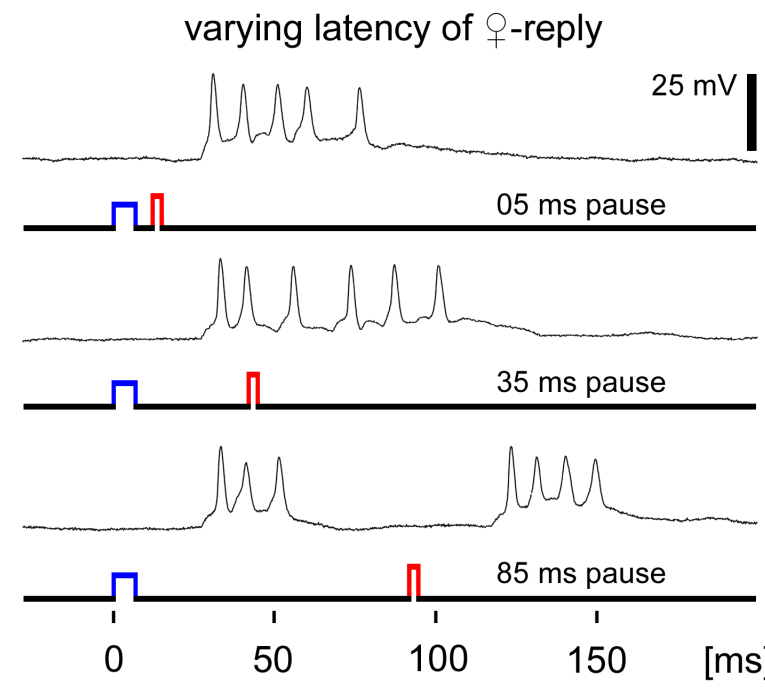

Figure 75: Recording examples of LBN7's response (depolarised) to the female answer (at $28 \mathrm{kHz}$ ) with varying pause duration between male trigger and female reply in an artificial duet $(5,35$, and $85 \mathrm{~ms}$ pause duration; see chapter 2.3.2 for stimulus paradigm). Blue indicates the male's natural carrier frequency and red the natural frequency of the female reply.

Spike latencies in LBN7. For $50 \mathrm{~ms}$ pulse durations shortest spike latencies were measured in LBN7 with $29.7 \pm 0.8 \mathrm{~ms}(\mathrm{n}=5 ; 28 \mathrm{kHz}, 90 \mathrm{~dB}$ SPL; depolarised; mean and SD). The shortest EPSP latency, however, is $21.6 \pm 1.4 \mathrm{~ms}$ at $28 \mathrm{kHz}$ and $70 \mathrm{~dB}$ SPL ( $n=5$; mean and SD). This strongly indicates a monosynaptic connection to an ascending interneuron. The response characteristics of LBN7 at all acoustical programs tested in this study closely match the response characteristics of AN2 (shortest spike latency of $21.7 \mathrm{~ms}$ in brain recordings of AN2; see chapter 3.1.2). 
This applies for the intensity dependence at various frequencies, the dependence on temporal parameters (especially the dependence on pulse duration) and the dependence on the carrier frequency of the female reply. Therefore, it is most likely that LBN7 is directly connected to AN2. However, in addition to the input of AN2, LBN7 receives inhibitory input from a different neuron. The latency of the inhibition could not be determined, though, since the beginning of the IPSP was always concealed by the preceding EPSP.

Secondary long latency response in LBN7. High intensity stimulation at various carrier frequencies evokes a relatively short latency spiking response in LBN7 (see above). Approximately 600 to $650 \mathrm{~ms}$ subsequent to these high intensity stimuli a secondary response of LBN7 can be observed (Figure 76).

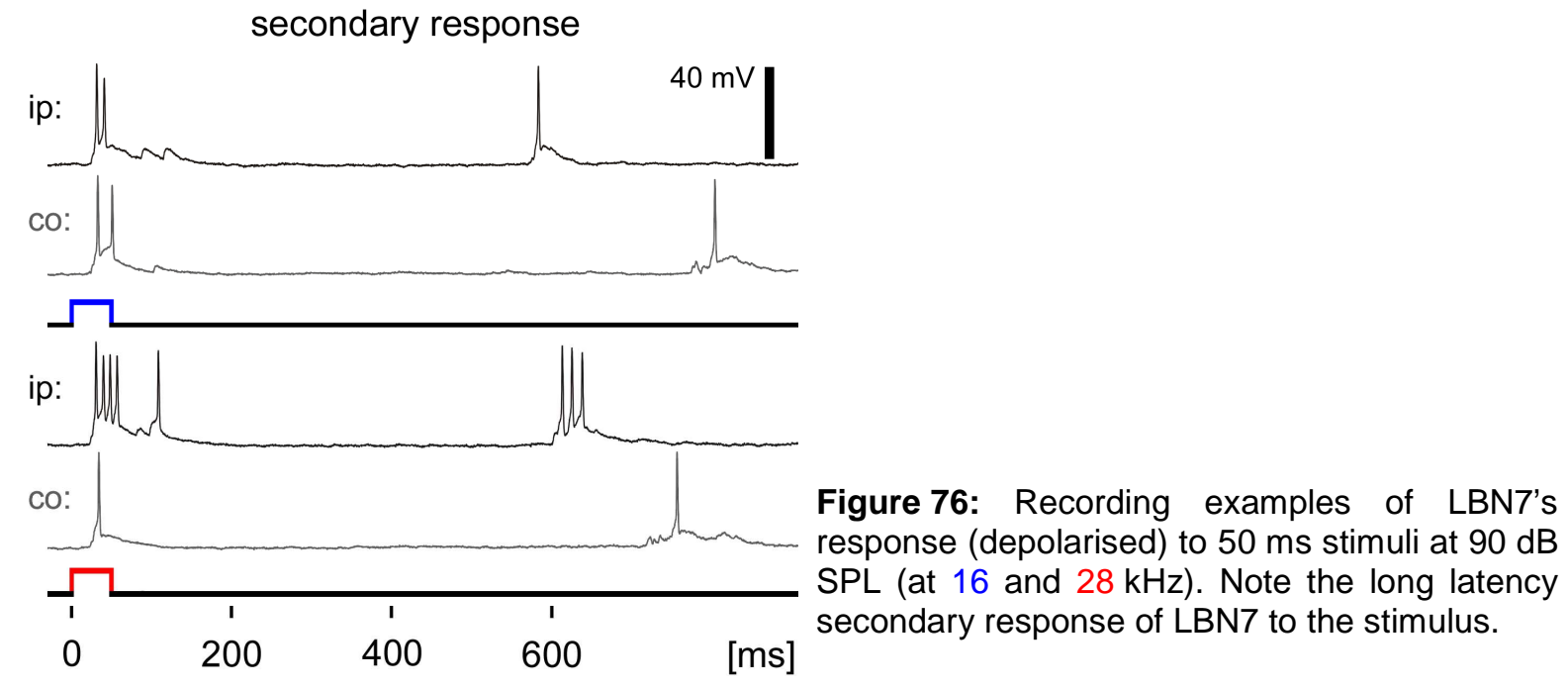

This secondary response consists of graded potentials that are able to reach the spiking threshold. The strongest response with a maximum of 3 spikes could be observed at 28 and $30 \mathrm{kHz}$ ( $50 \mathrm{~ms}$ pulse duration, $90 \mathrm{~dB} \mathrm{SPL}$ ). Also with stimulation from the contralateral side of penetration this secondary response can be observed, but the latency of the graded potential and the spikes are increased by approximately $100 \mathrm{~ms}$. 


\subsubsection{Crossing local brain neuron 8 (cLBN8)}

An interneuron that is often recorded in the lateral protocerebrum is cLBN8. Because of its low spiking activity a clear classification was only achieved by its characteristical morphology. Thus, the data presented in this chapter only stems from cLBN8s where morphology has been verified by a staining.

Morphology. In Figure 77 the morphology of CLBN8 from three different individuals is displayed. In the left and middle brain the complete morphology is given, whereas only the soma ipsilateral arborisations were stained in the right brain (nevertheless, a clear axon running to the contralateral side could be seen). The soma is always located underneath the anterior lateral surface of the brain (in more dorsal regions) close to the entrance of the optical lobe.

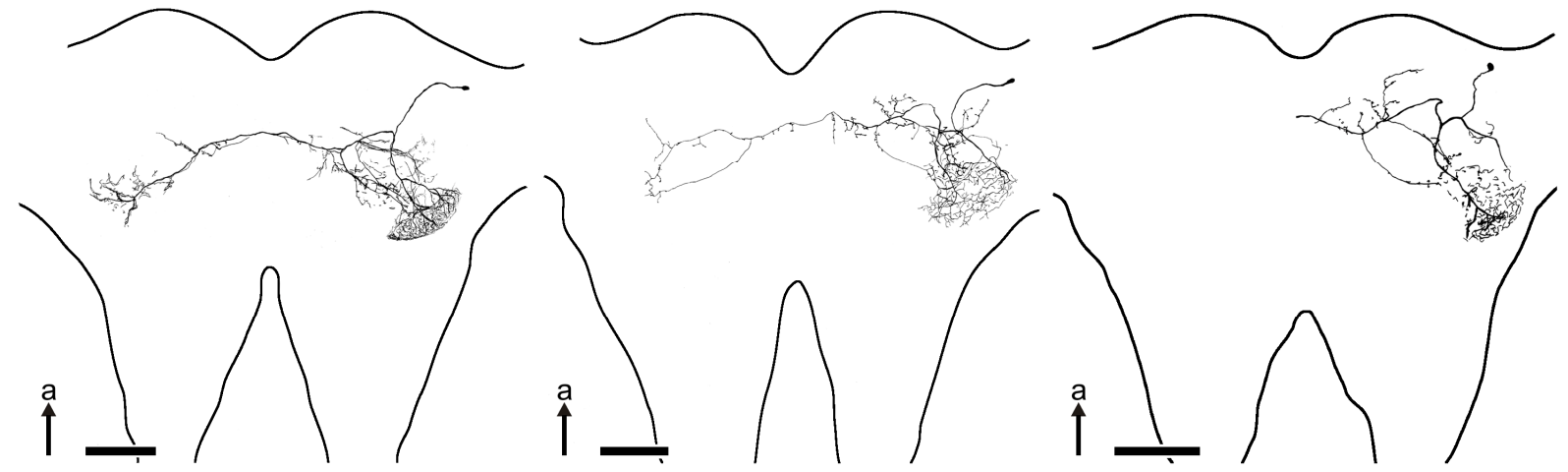

Figure 77: Morphology of CLBN8 in the brain of A. nigrovittata assured in 3 individuals (in the right drawing only arborisations ipsilateral to the soma were seen). Bar represents $200 \mu \mathrm{m}$. $\mathrm{a}=$ anterior.

The primary neurite extends medially posterior and sometimes also to more dorsal portions of the brain. Then it splits into two major branches. One branch runs to posterior lateral regions and arborises in ventral direction. The other major branch may slightly extend to lateral portions until it divides into two branches or may split already at the end of the primary neurite. One of these branches extends to posterior portions and may have a few more or less thick collaterals. This branch (and its collaterals) exhibits dense arborisations along the posterior border of the lateral protocerebrum in dorsal and ventral direction. All arborisations described to this point have smooth endings indicating to be postsynaptic. The other branch extents slightly to anterior ventral portions until it changes its direction directly running to the lateral protocerebrum of the other half of the brain. On its way a few ramifications are found that have beaded endings indicating to be presynaptic. At least one minor branch (sometimes more) splits off and extends back into the direction of the dendritic 
arborisations. This minor branch, though, runs in more ventral portions of the brain and thus, its sparse axonal arborisations are situated mostly in a different layer than the dendritic ramifications. The main axon, however, crosses the midline of the brain anterior to the central body. In the soma-contralateral side of the brain the axon gives off a collateral (at approximately the same position - in the other side of the brain though - where soma-ipsilateral the minor axonal branch extended back to the dendritic arborisations). The collateral runs to lateral slightly posterior portions and arborises sparsely in ventral direction. The main axon, on the other hand, extends to lateral portions and also arborises sparsely in ventral direction.

Intensity and carrier frequency dependence of $C L B N 8$ responses. Presentation of an artificial duet evokes obvious EPSPs in cLBN8 to the male calling song and to the female reply (Figure 78A). Spiking occurred occasionally to the female reply only. The overall spiking activity to standard stimuli was also very low for all carrier frequencies (Figure 78B). The strongest response, however, was found for $28 \mathrm{kHz}$ at $40 \mathrm{~dB}$ SPL although the spike number differs greatly between the individuals (note the high standard error). When considering the mean response strength of the individuals, the strongest response was always found at this carrier frequency and intensity ( $28 \mathrm{kHz}$ at $40 \mathrm{~dB} \mathrm{SPL}$; Figure $78 \mathrm{C}$ ). A similar maximum in response for standard stimuli can be found in ANx (see Figure 30A).

Latency and intensity dependence at various frequencies of cLBN8's EPSP. In Figure 79 the latencies of the EPSPs in CLBN8 are displayed for all frequencies of the standard stimuli. The shortest latencies can be observed for stimuli to both ultrasonic frequencies. EPSP latencies to $16 \mathrm{kHz}$ stimulation are increased by about $3 \mathrm{~ms}$ at all intensities tested when compared to $28 \mathrm{kHz}$. The shortest latencies, however, were found for $28 \mathrm{kHz}$ at $80 \mathrm{~dB}$ SPL with $19.4 \pm 0.2 \mathrm{~ms}(\mathrm{~N}=4$; mean and $\mathrm{SE}$ ). Such short latencies strongly suggest a monosynaptic coupling to an ascending interneuron. The shortest spike latencies of cLBN8 were measured for $28 \mathrm{kHz}$ at $70 \mathrm{~dB}$ SPL with $31.6 \pm 4.2 \mathrm{~ms}$ ( $\mathrm{N}=4$; mean and $\mathrm{SE}$ ). 

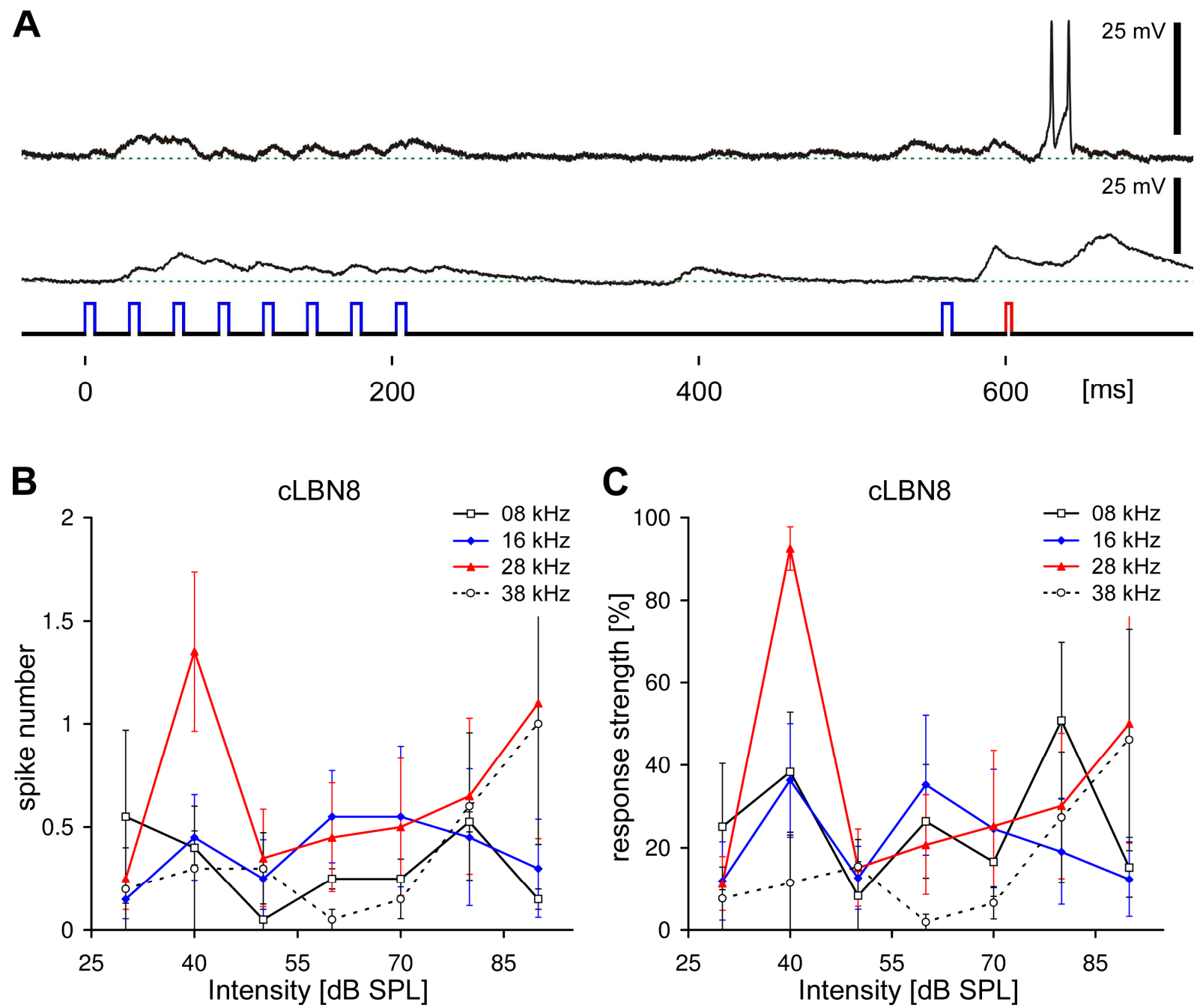

Figure 78: Response of cLBN8 to acoustic stimuli. Blue colouration - carrier frequency of the male song $(16 \mathrm{kHz})$ and red colouration - carrier of the female reply $(28 \mathrm{kHz})$. A: Two example recordings of the response to an artificial duet of $A$. nigrovittata (male song at $70 \mathrm{~dB}$ SPL; female answer at $60 \mathrm{~dB}$ SPL; see chapter 2.3.2 for stimulus paradigm). B: Spike numbers of cLBN8 to standard stimuli ( $\mathrm{N}=4$; means and SE). C: Response strength of cLBN8 to standard stimuli ( $N=4$; means and SE).

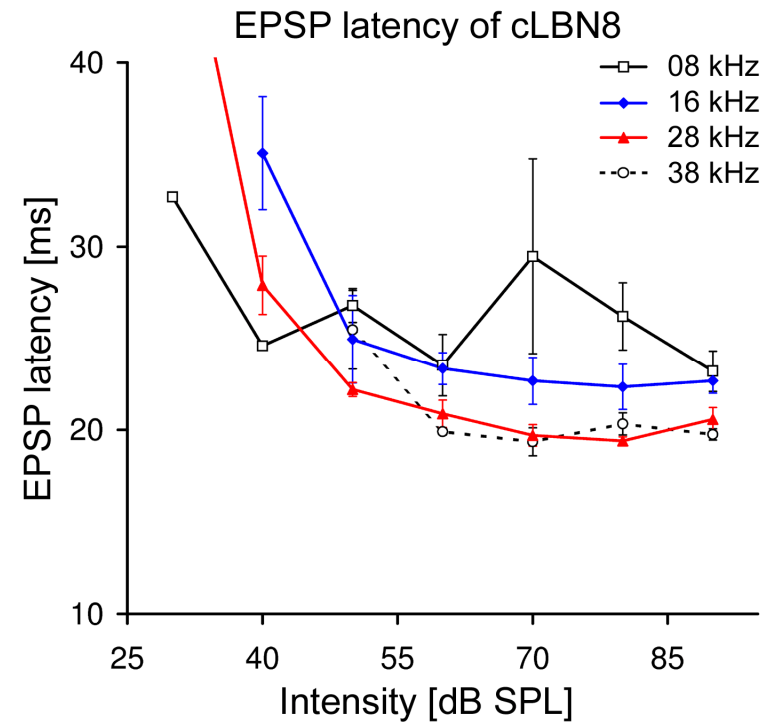

Figure 79: EPSP latencies in CLBN8 to standard stimuli ( $\mathrm{N}=4$; means and $\mathrm{SE})$. 
For one example also the EPSP amplitudes could be measured from the original recording data obtained during the presentation of standard stimuli (Figure 80A). A lowest threshold for graded potentials in cLBN8 can be seen for $28 \mathrm{kHz}$ below $40 \mathrm{~dB}$ SPL. The thresholds for stimuli with 16 and $38 \mathrm{kHz}$ was found to be $10 \mathrm{~dB}$ higher. The overall intensity dependence of cLBN8's EPSP to standard stimuli is similar to the spiking responses of TN1 (Figure 80B). Both neurons show a low response to stimuli with $8 \mathrm{kHz}$. At $28 \mathrm{kHz}$ a response peak is found at $40 \mathrm{~dB} \mathrm{SPL}$ (respectively $50 \mathrm{~dB} S P L$ for $38 \mathrm{kHz}$ ) that is followed by a slight decrease in activity and an increase to higher intensities. Such characteristical response to ultrasonic frequencies has not been found in any ascending neuron except TN1. When comparing the shortest latencies of cLBN8's EPSPs (19.4 $\pm 0.2 \mathrm{~ms}$ - see above) to the shortest latencies of TN1 (19.5 $\pm 0.6 \mathrm{~ms}$ - see chapter 3.1.5) a direct connection between both neuron seems likely (note that the closely corresponding latencies of TN1-spikes and cLBN8-EPSPs were obtained in different individuals). Thus, despite a similar spiking activity of cLBN8 and ANx, a connection between both neurons seems unlikely. Furthermore, the shortest spike latency of ANx with $24.9 \pm 0.6 \mathrm{~ms}$ (see chapter 3.1.6) is too long to release an EPSP with a shorter latency in cLBN8.
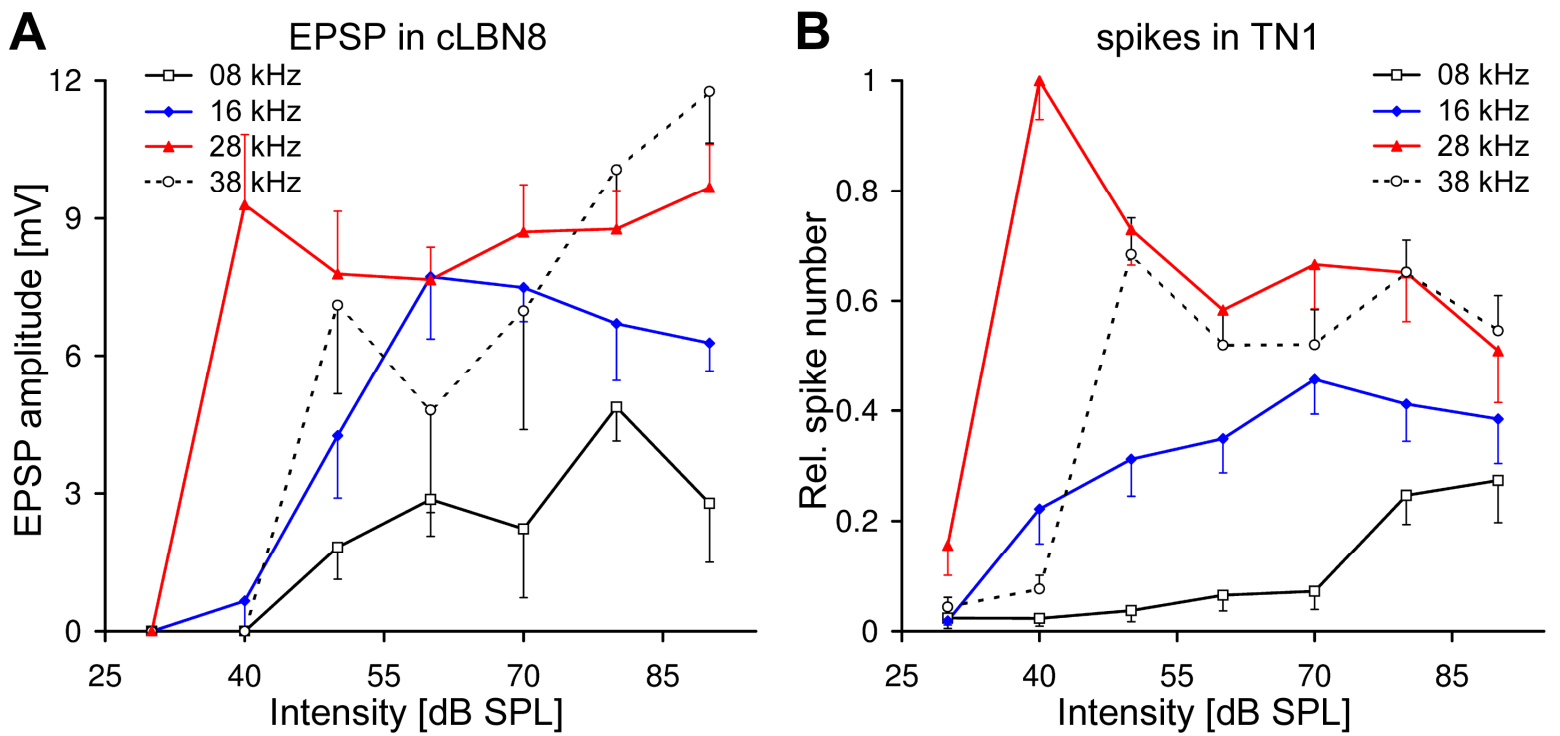

Figure 80: Responses to standard stimuli. Blue colouration - carrier frequency of the male song $(16 \mathrm{kHz})$ and red colouration - carrier of the female reply $(28 \mathrm{kHz})$. A: EPSP amplitude of the EPSP in cLBN8 ( $n=5$; means and SD). B: Relative spike number of TN1 ( $N=15-16$; means and SE).

Responses of cLBN8 to varying temporal parameters. Stimulation of cLBN8 with white noise stimuli of varying temporal pattern shows no obvious dependency on pause duration (Figure 81A). 

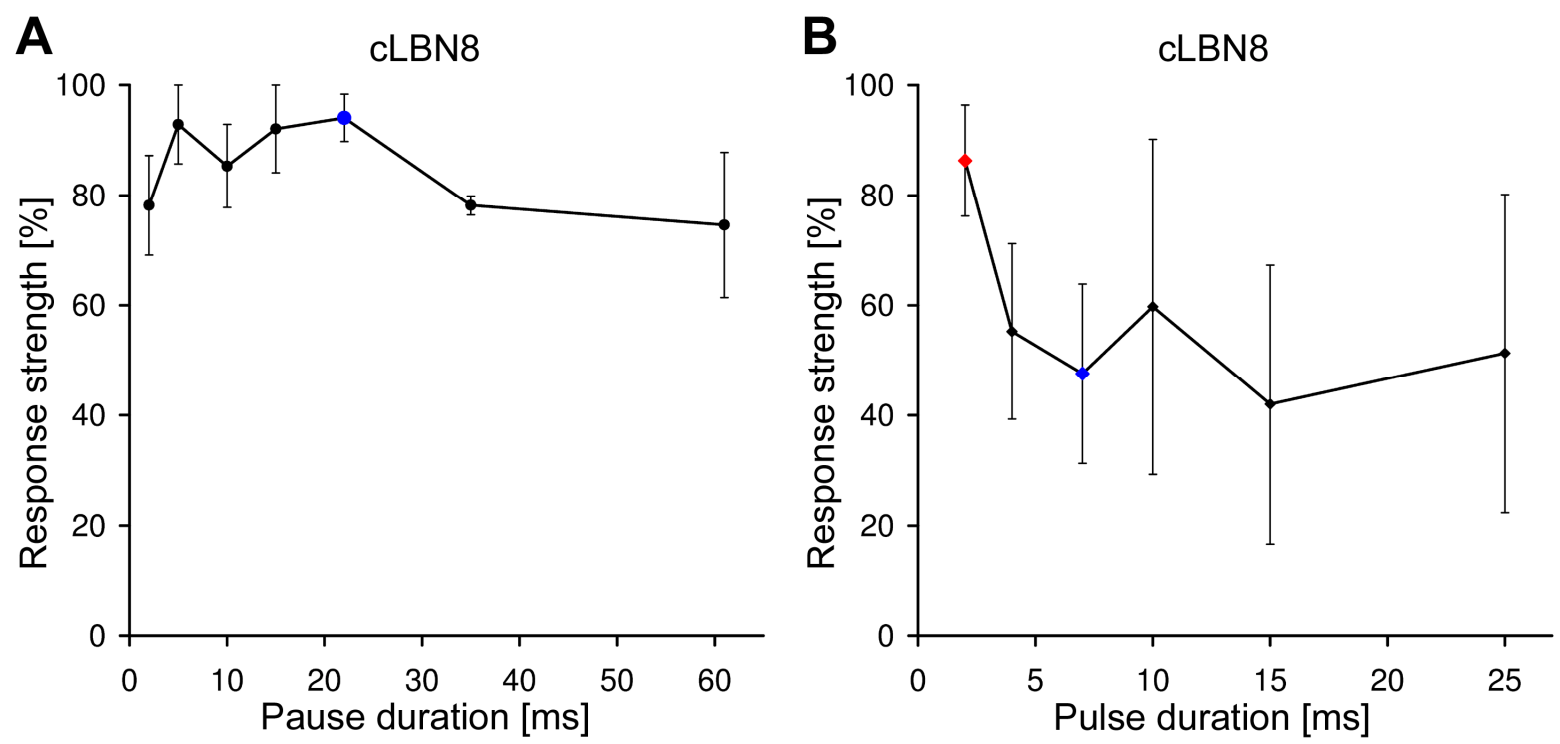

Figure 81: Response strength of CLBN8 (depolarised) to different temporal parameters (white noise; $70 \mathrm{~dB}$ SPL; see chapter 2.3.2 for stimulus paradigm; means and SE). Important natural parameters are emphasised (blue: male song pause/pulse duration; red: female reply pulse duration). A: Magnitude of response to stimuli with varying pause duration $(\mathrm{N}=3)$. B: Magnitude of response to stimuli with varying pulse duration $(\mathrm{N}=3)$.

The highest spike numbers can be seen at $22 \mathrm{~ms}$ pause duration with $3.3 \pm 1.3$ spikes $(\mathrm{N}=3$; mean and $\mathrm{SE})$. At varying pulse durations the strongest response of CLBN8 is found for stimuli with the shortest pulse duration tested $(2 \mathrm{~ms}$; pulse duration of the female reply) although the maximum spike number is low $(2.1 \pm 0.7$ spikes - $\mathrm{N}=3$; mean and SE; Figure 81B). This increase in response to short pulse durations can also be observed in TN1 (see chapter 3.1.5).

Response to different frequencies of the female answer in an artificial duet. When presenting an artificial duet with varying carrier frequencies of the female reply the strongest spiking activity is revealed for $24 \mathrm{kHz}$ (Figure 82). The response to $28 \mathrm{kHz}$ seems to be reduced although a high intraindividual variability is evident for all ultrasonic frequencies above $24 \mathrm{kHz}$. A strong decrease in response, however, can be clearly observed for sonic frequencies (with a small standard error) and can also be found in the responses of TN1 to the same stimulus paradigm (see Figure 24). 


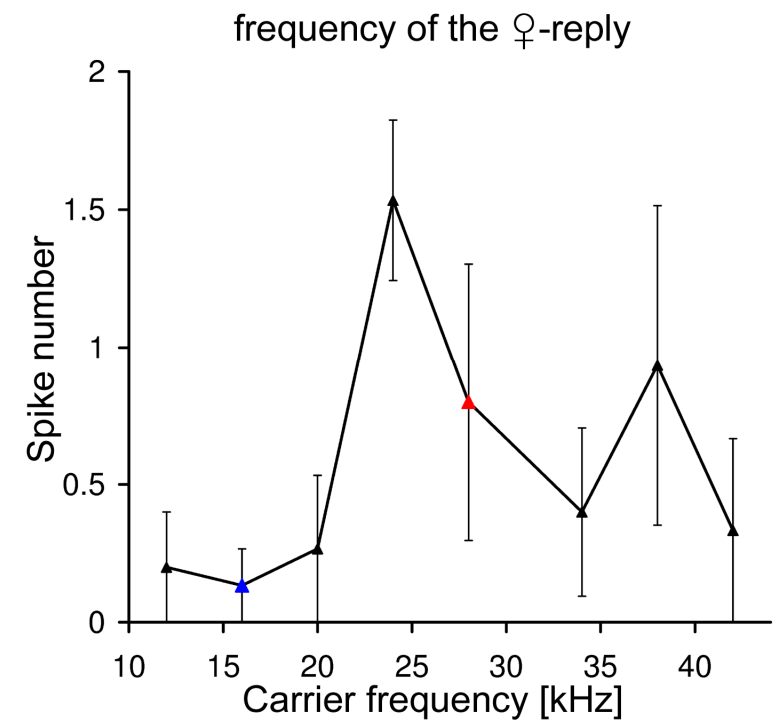

Figure 82: Response of CLBN8 to the female answer with varying carrier frequency in an artificial duet (see chapter 2.3.2 for stimulus paradigm). Blue indicates the male's natural carrier frequency and red the natural frequency of the female reply $(\mathrm{N}=3$; means and $\mathrm{SE})$.

Inhibition of CLBN8's response at ultrasonic frequencies. In one intracellular recording of a depolarised cLBN8 also IPSPs were found subsequent to the excitation (Figure 83). These IPSPs had a comparatively long latency of $51.8 \pm 1.5 \mathrm{~ms}$ (for $46 \mathrm{kHz}$ at $90 \mathrm{~dB} \mathrm{SPL} ; \mathrm{n}=5$; mean and SD) and a long duration of $209.1 \pm 34.0 \mathrm{~ms}$ $(n=19$; mean and SD; arrows mark the beginning and the end of the IPSP in Figure 83). In the original recordings of CLBN8 it was clearly visible at $90 \mathrm{~dB} S P L$ for all ultrasonic frequencies. Only for $46 \mathrm{kHz}$ the IPSP was already seen at $80 \mathrm{~dB}$ SPL. At lower intensities the IPSP may disappear in the compound potential but may influence the spiking activity for ultrasonic frequencies of cLBN8.

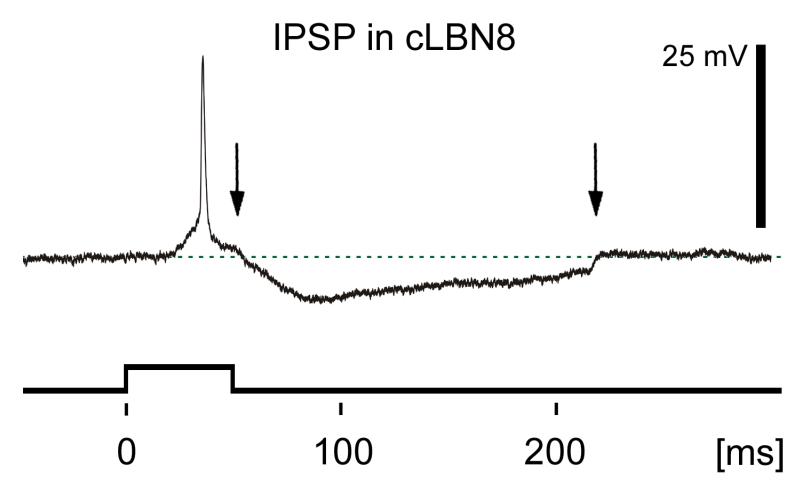

Figure 83: Example recording of the response of cLBN8 to a $50 \mathrm{~ms}$ pulse at $90 \mathrm{~dB}$ SPL $(46 \mathrm{kHz}$; depolarised). Note the long duration of the IPSP (beginning and end marked by the arrows). 


\subsubsection{Local brain neuron 9 (LBN9)}

Another interneuron that has been recorded five times in the brain of A. nigrovittata is LBN9. The characteristical response to an artificial duet separates LBN9 from other auditory brain neurons and makes a definite classification possible.

Morphology. One complete staining shows the morphology of LBN9 which is displayed in Figure 84. The overall extent is restricted to one half of the brain (Figure 84A). The soma of LBN9 is situated underneath the dorsal surface in the lateral protocerebrum of the brain. Its primary neurite runs anterior, then posterior and slightly lateral on its way to more ventral portions of the brain. Then the primary neurite divides into two major branches (see detailed view of LBN9 in Figure 84B). One branch extends to anterior portions whereby dense arborisations are given off to dorsal as well as to ventral portions. These arborisations seem to be postsynaptic due to its smooth endings. The other major branch extends medially anterior, in ventral direction without any arborisations. Then it runs laterally and ramifications are found with beaded endings indicating to be the axonal part of LBN9. The major axon divides into two minor axons. One of the minor axons runs anteriorly and gives off dense arborisations. The other minor branch extends to lateral posterior portions and gives off sparse arborisations.

A
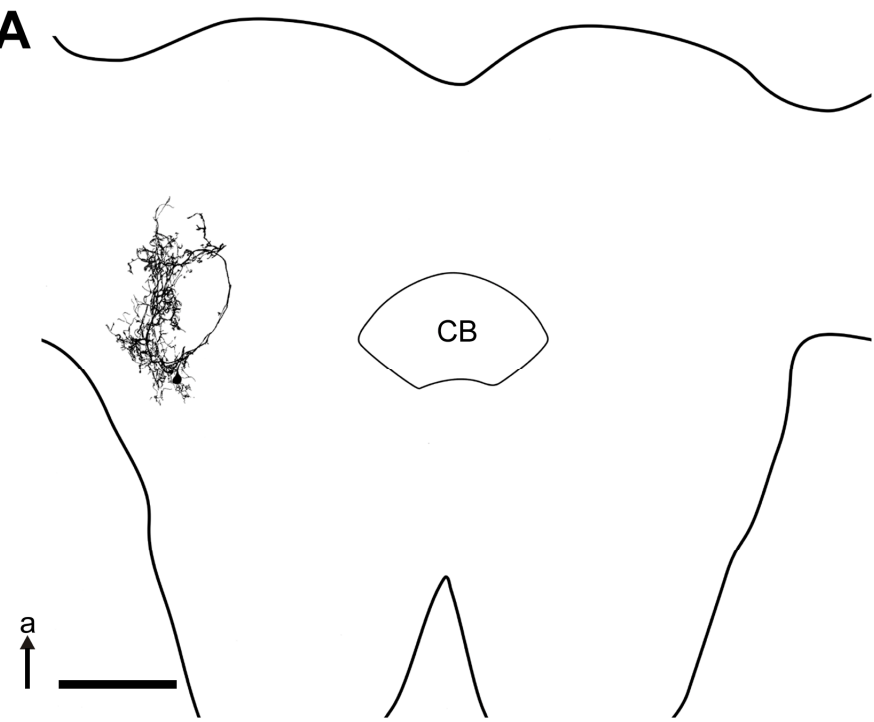

B

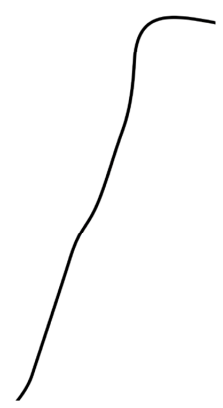

B

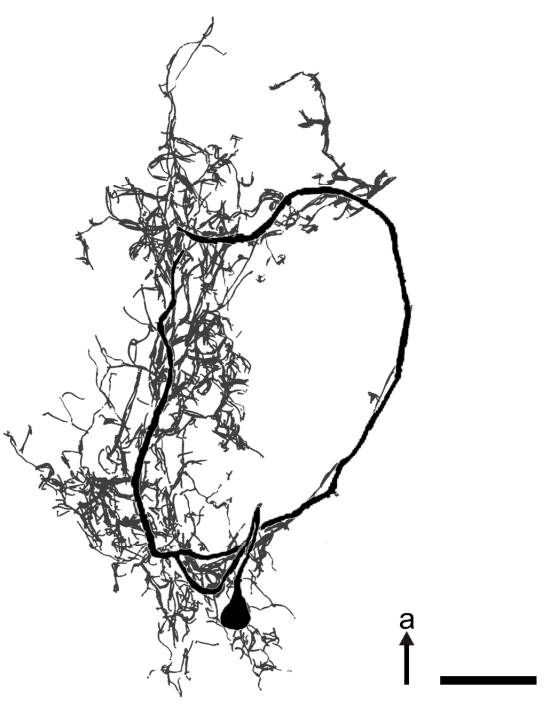

Figure 84: Morphology of LBN9 in the brain of $A$. nigrovittata. A: Horizontal overview of LBN9 after a Cy3-staining in relation to the central body (CB). Bar represents $200 \mu \mathrm{m}$. B: Detailed view on LBN9, at which major branches are emphasised. Bar represents $50 \mu \mathrm{m}$. a=anterior. 
Intensity and carrier frequency dependence of $L B N 9$ responses. The response to an artificial duet is very characteristical (Figure 85A). The calling song of the male elicits a strong IPSP whereas the female reply elicits a strong EPSP in LBN9. In the average of five repetitions (lower trace in Figure 85A) one can see the whole duration of the IPSP that may last for several hundred milliseconds (marked by the arrows in Figure 85A). Spiking occurred to the female reply only. Responses to $50 \mathrm{~ms}$ pulses of the standard stimuli were low at all frequencies tested (Figure 85B). Stimulation with an artificial duet and varying carrier frequency, however, revealed a highest spike numbers at 24 and $28 \mathrm{kHz}$ (Figure 85C).

A
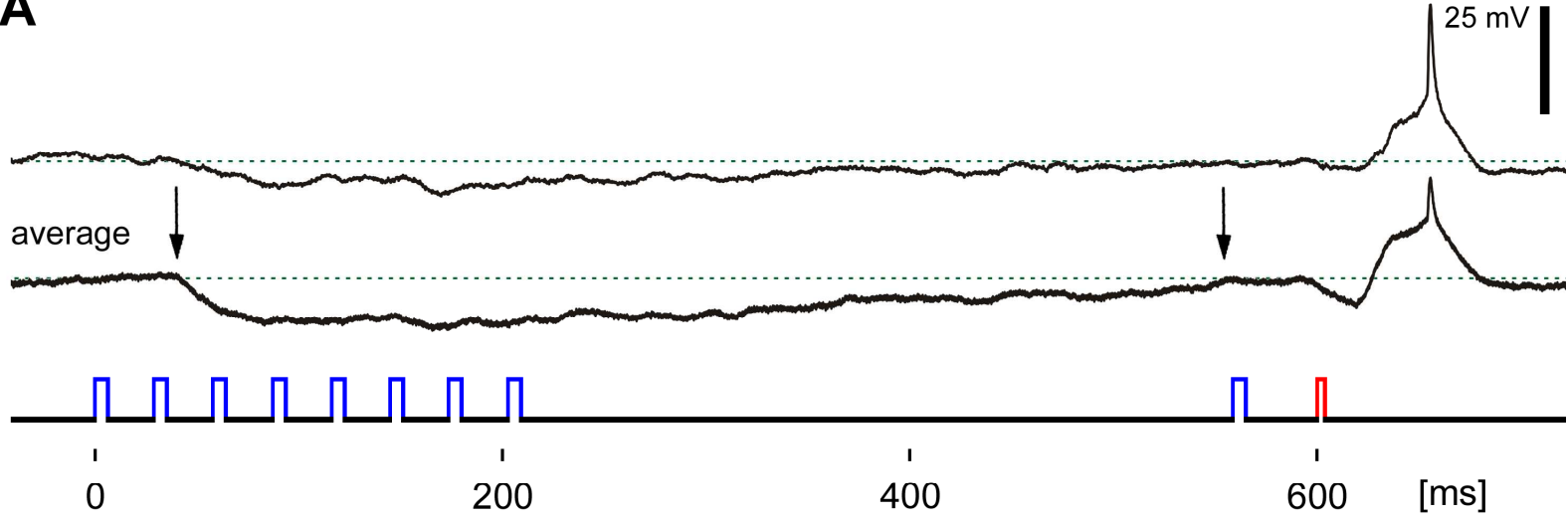

B

$50 \mathrm{~ms}$ pulses
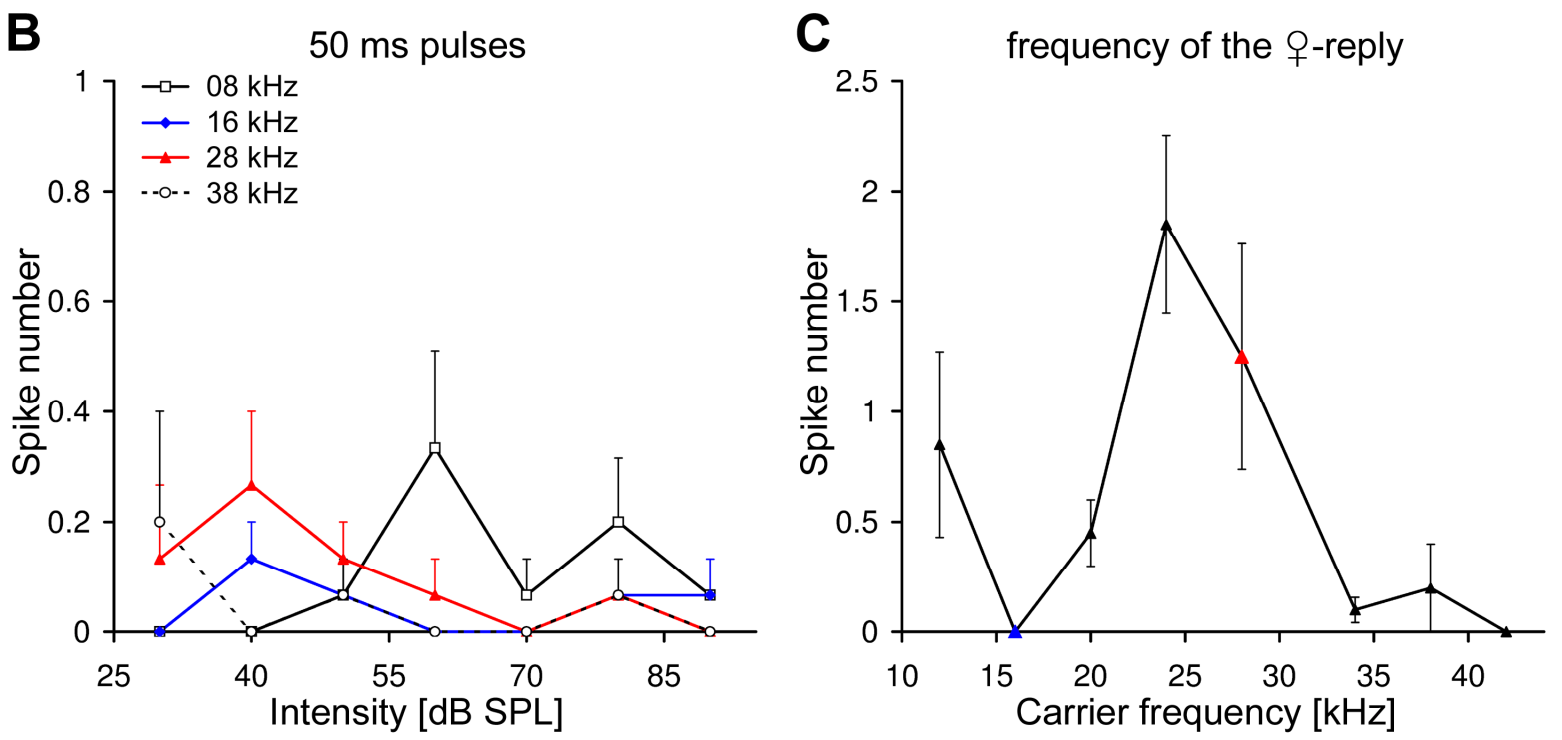

Figure 85: Response of LBN9 to acoustic stimuli. Blue colouration - carrier frequency of the male song $(16 \mathrm{kHz})$ and red colouration - carrier of the female reply $(28 \mathrm{kHz})$. A: Example recording of the response to an artificial duet of $A$. nigrovittata (male song at $70 \mathrm{~dB}$ SPL; female answer at $60 \mathrm{~dB}$ SPL; see chapter 2.3.2 for stimulus paradigm). Upper trace shows a single recording whereas the lower trace shows an average of 5 repetitions. The arrows mark the beginning and the end of the inhibition. B: Response of LBN9 to standard stimuli ( $N=3$; means and SE). C: Response to the female answer with varying carrier frequency in an artificial duet $(\mathrm{N}=4$; see chapter 2.3.2 for stimulus paradigm; means and SE). 
IPSP and EPSP dependence on carrier frequency and intensity during stimulation with the male calling song. In Figure 86 averaged recordings of LBN9 to the male calling song with varying frequency and intensity are displayed. At low intensities (50 dB SPL) a strongest spiking response can only be found at $28 \mathrm{kHz}$ (marked by an asterisk). At $70 \mathrm{~dB}$ SPL IPSPs can be clearly seen in the response to sonic frequencies. At the same intensity only compound potentials can be seen at the responses to 28 and $38 \mathrm{kHz}$. Contrary to $70 \mathrm{~dB}$ SPL, at $90 \mathrm{~dB}$ SPL strongest IPSPS can be observed in the response to 28 and $38 \mathrm{kHz}$. The IPSP at the sonic frequencies, on the other hand, seems to be reduced. Also excitation can is visible in the averaged recordings (marked by the arrows) that is pronounced in the activity of LBN9 for all frequencies with $90 \mathrm{~dB}$ SPL stimulation. The EPSP always precedes the IPSP and has a shortest latency of $19.8 \pm 1.5 \mathrm{~ms}$ (at $28 \mathrm{kHz}$ and $80 \mathrm{~dB}$ SPL; measured at the responses to standard stimuli; $n=5$; mean and SD). This indicates a direct coupling to an ascending interneuron. The IPSP has a shortest latency of $28.4 \pm 1.8 \mathrm{~ms}$ for $16 \mathrm{kHz}$ at $60 \mathrm{~dB} \mathrm{SPL}$ (measured at the responses to standard stimuli; $n=5$; mean and SD), which indicates the involvement of at least one additional interneuron in the inhibitory pathway to LBN9. The latency of the IPSP to the beginning of the male calling song is markedly longer with $45.9 \pm 1.1 \mathrm{~ms}(16 \mathrm{kHz}$ at $70 \mathrm{~dB}$ SPL; $\mathrm{N}=3$, mean and SE). Due to the interaction of the EPSP and IPSP in the compound potential, no absolute amplitudes could be measured.

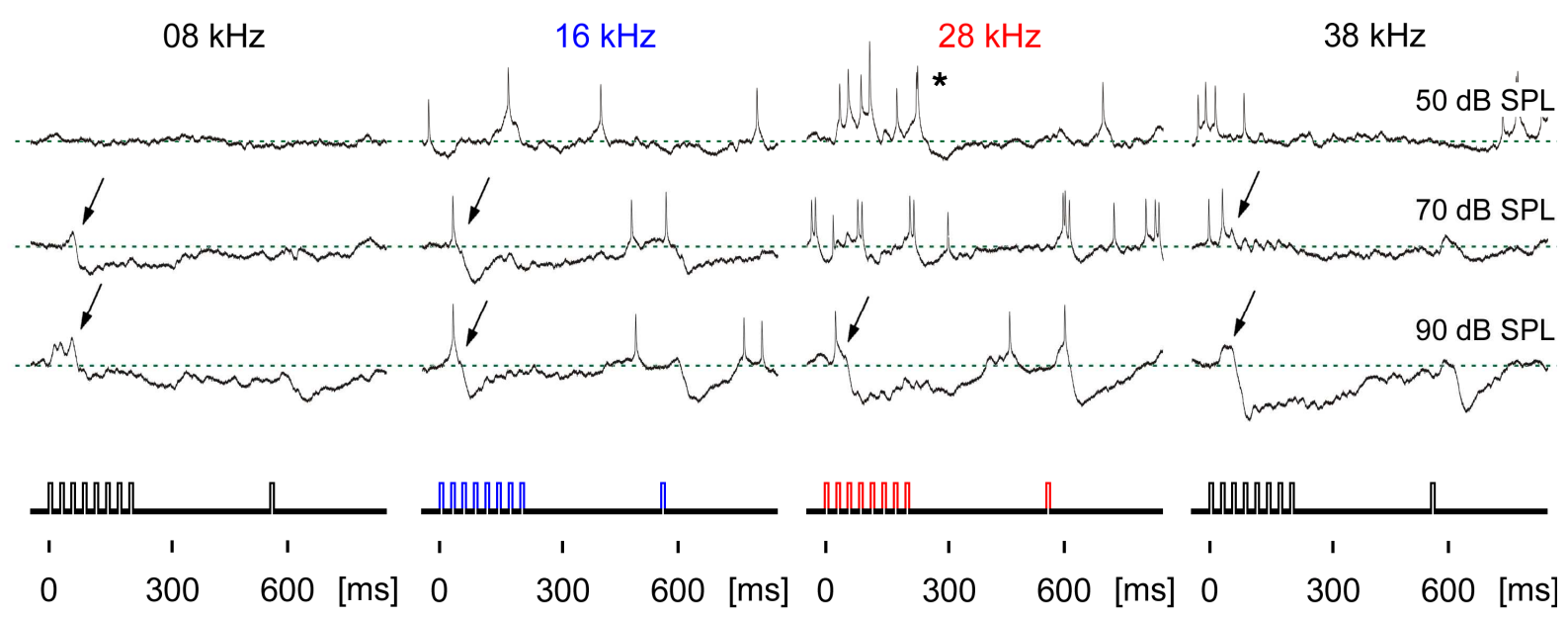

Figure 86: Averaged recordings (of 5 repetitions) of LBN9 to the male calling song for 8, 16, 28 and $38 \mathrm{kHz}$ at 50, 70 and $90 \mathrm{~dB}$ SPL (see chapter 2.3.2 for parameters of the stimuli). The asterisks mark the increased response of LBN9 to $28 \mathrm{kHz}$ stimuli at low intensities. The arrows mark clearly visible EPSPs preceding the IPSP. Blue colouration - carrier frequency of the male song $(16 \mathrm{kHz})$ and red colouration - carrier of the female reply $(28 \mathrm{kHz})$. 
Time constants of the IPSP and EPSP in LBN9. In Figure 87 averaged responses of two individuals are displayed to stimulation with an artificial duet and varying carrier frequency of the female reply. It has already been shown that the inhibition in LBN9 may last for several hundred milliseconds (also see Figure 85A). Such long time constants can also be observed when stimulating with the female answer at $24 \mathrm{kHz}$. The graded potential slowly decreases in amplitude and continues until the beginning of the subsequent verse of the duet. The EPSP may then even get "aborted" by the beginning of the IPSP (see arrow in Figure 87). This has not been observed with stimulation of other carrier frequencies than $24 \mathrm{kHz}$. At $28 \mathrm{kHz}$ only a small EPSP can be seen that is short in duration (compare with the grey overlay of the $24 \mathrm{kHz}$-stimulation). For stimulation with $16 \mathrm{kHz}$ no EPSPs gets elicited but a slight IPSP is evident. Such a long duration response (as seen at $24 \mathrm{kHz}$ ) has never been observed from an ascending interneuron although the EPSP latencies strongly suggest LBN9 to be a second order interneuron in the excitatory pathway.

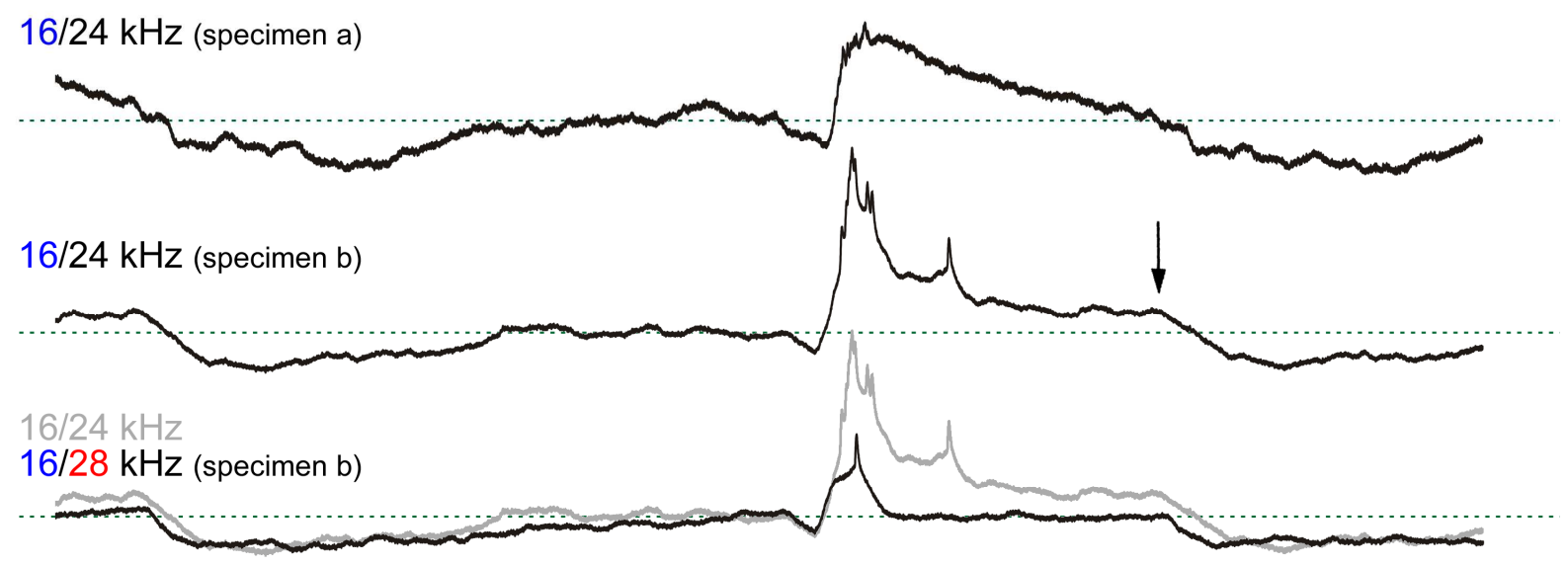

$16 / 16 \mathrm{kHz}$ (specimen b)

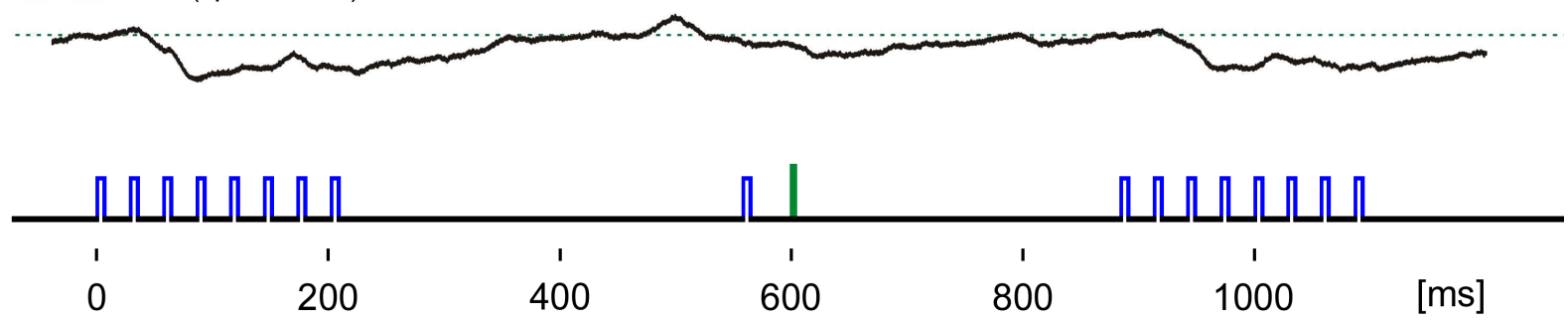

Figure 87: Averaged recordings (5 repetitions) of LBN9 to an artificial duet with varying carrier frequency of the females reply (see chapter 2.3.2 for parameters of the stimuli). The arrow marks the "abortion" of the EPSP to the female reply by the IPSP of the subsequent verse. Grey overlay at $28 \mathrm{kHz}$ shows the different amplitudes of the EPSPs to 28 and $24 \mathrm{kHz}$ stimuli. Blue colouration carrier frequency of the male song $(16 \mathrm{kHz})$ and red colouration - carrier of the female reply $(28 \mathrm{kHz})$. 


\subsubsection{Local brain neuron 10 (LBN10)}

LBN10 is an interneuron that has been recorded twice. Characteristically, high spontaneous activity can be seen during the intracellular recording that gets inhibited by acoustical stimulation. The morphological extent of LBN10 could not be fully obtained but a faint Cy3-staining supposes LBN10 to be restricted to one half of the brain with its soma situated near the anterior lateral surface of the brain, close to the anterior entrance of the optical lobe.

Responses of LBN10 to an artificial duet and to standard stimuli. Stimulation with an artificial duet evokes strong inhibitory postsynaptic potentials in LBN10 to both, the male calling song and to the female answer (Figure 88). Although the inhibition to the male's pulse series lasts until shortly before the trigger pulse, spikes can be recorded when the potential has not even reached the level of the resting membrane potential.

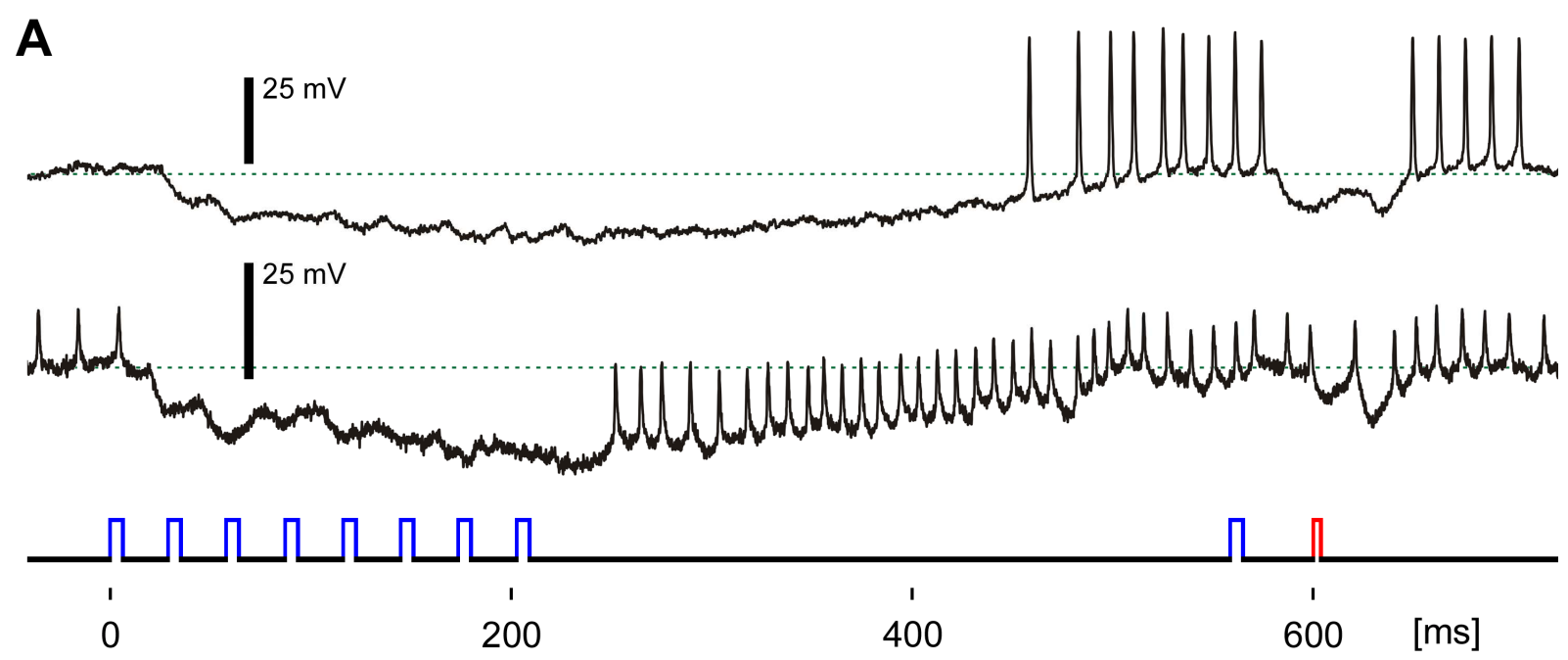

Figure 88: Two example recordings of LBN10 to an artificial duet of $A$. nigrovittata (male song at $70 \mathrm{~dB}$ SPL; female answer at $60 \mathrm{~dB}$ SPL; see chapter 2.3.2 for stimulus paradigm). Blue colouration carrier frequency of the male song $(16 \mathrm{kHz})$ and red colouration - carrier of the female reply $(28 \mathrm{kHz})$.

When stimulating with standard stimuli the frequency dependence of the IPSP becomes evident (Figure 89). At $50 \mathrm{~dB}$ SPL a strong IPSP with a short duration can be observed at $28 \mathrm{kHz}$. At $16 \mathrm{kHz}$ the IPSP has a comparatively longer duration. This indicates that possibly the output of two presynaptic neurons are responsible for the IPSPs in LBN10. One of these neurons may exhibit a tonic response that causes the IPSP with the long duration, whereas the other neuron may have phasic spiking activity that causes the IPSP with the short duration. With increasing intensities this 
multiple inhibition becomes more obvious. At $28 \mathrm{kHz}$ the tonic IPSP increases in amplitude and mixes with the phasic IPSP. This leads to a biphasic appearance of the IPSP in the intracellular recording. The same is true for stimuli with $16 \mathrm{kHz}$ carrier frequency at increasing intensities. Here, the phasic IPSP (that is already present at $50 \mathrm{~dB} \mathrm{SPL}$ ) gains in amplitude, which also leads to the biphasic appearance. Responses of LBN10 for $38 \mathrm{kHz}$ at low intensities only show inhibition by the phasic input. At $38 \mathrm{kHz}$ also the tonic input increases. At $8 \mathrm{kHz}$ inhibition of the response is mainly evident at high intensities only.

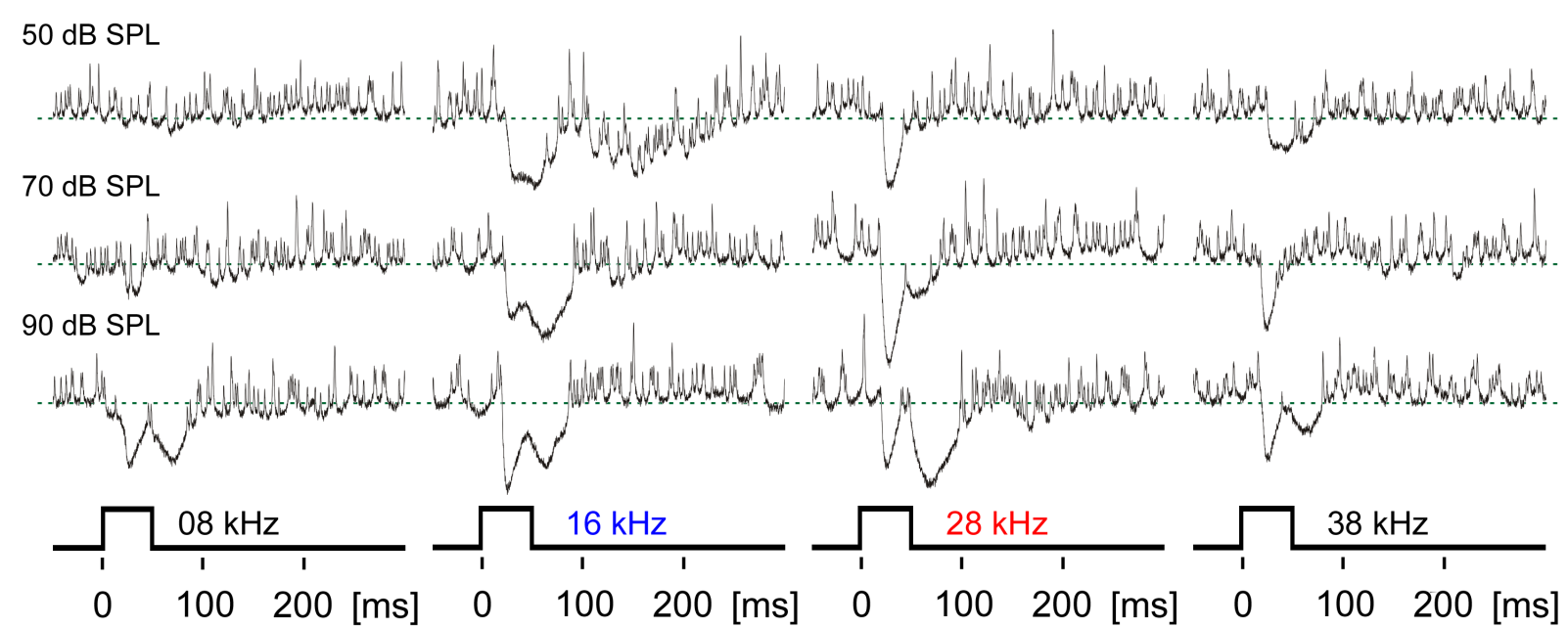

Figure 89: Averaged example recordings $(n=5)$ of LBN10 to standard stimuli at 50,70 and $90 \mathrm{~dB}$ SPL. Note the two independent IPSPs in LBN10.

IPSP latencies and characteristics. The phasic IPSP exhibits short latencies. The shortest latencies were found for $28 \mathrm{kHz}$ at $90 \mathrm{~dB}$ SPL with $20.1 \pm 2.4 \mathrm{~ms}(\mathrm{~N}=2, \mathrm{n}=8$; mean and SD). Such short latencies suggest an ascending interneuron to be the candidate for the inhibitory input in LBN10. In order to reveal the characteristics of the phasic inhibition, the relative amplitudes of the IPSPs were measured from the averaged responses of LBN10 (Figure 90A; dashed lines represent the single responses for 16 and $28 \mathrm{kHz}$ ). Lowest thresholds of the phasic IPSP were obtained for $28 \mathrm{kHz}$ at $40 \mathrm{~dB}$ SPL with a saturating type of response for increasing intensities. For 16 and $38 \mathrm{kHz}$ the threshold is $10 \mathrm{~dB}$ higher but the saturating characteristics are also evident. The relative IPSP amplitude to $8 \mathrm{kHz}$ stimuli increases at high intensities only. Similar response characteristics are found in TN1 recorded in the brain (Figure 90B) although a closer correspondence is found with TN1 recorded in the thorax (see Figure 19 left). 

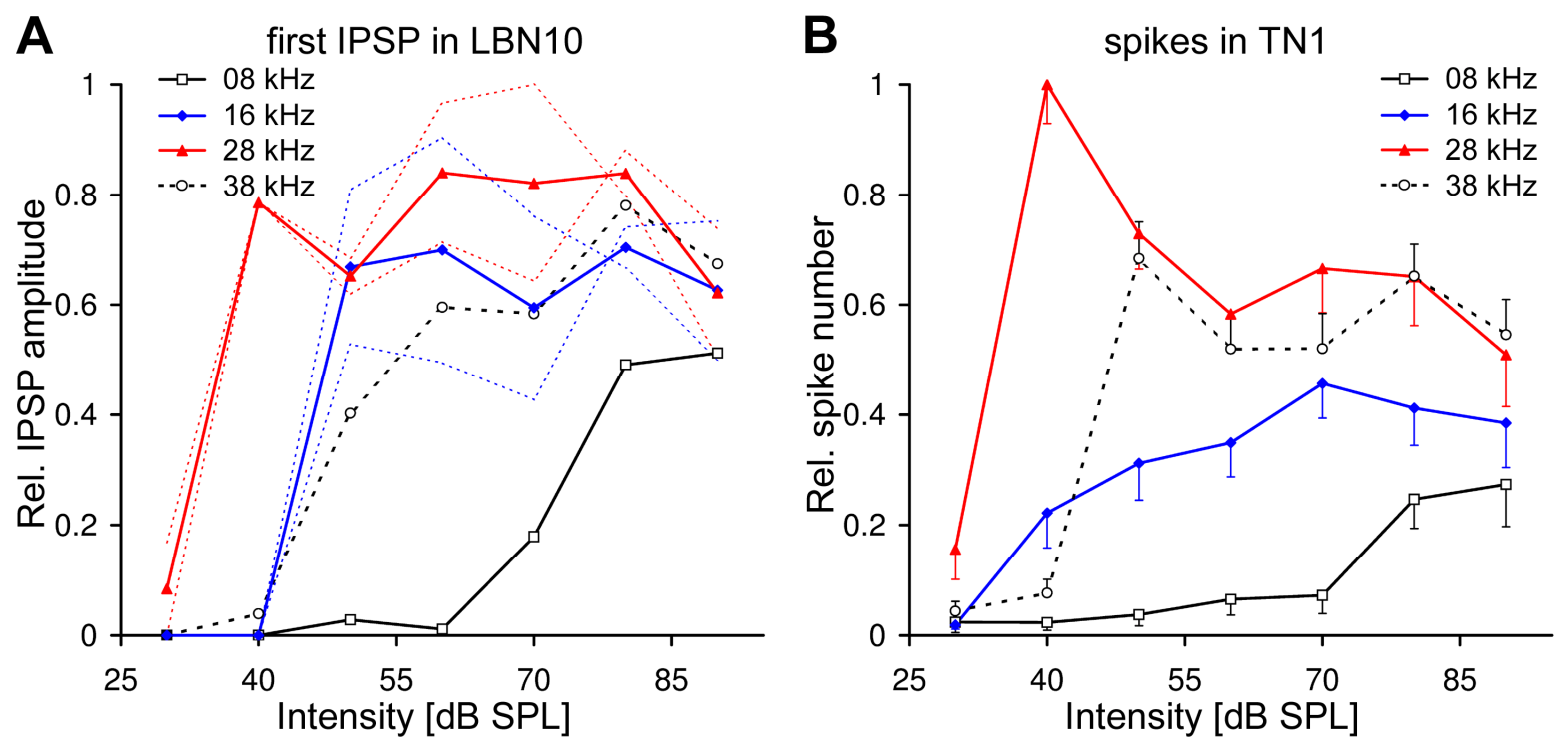

Figure 90: A: Relative amplitude of the IPSP in LBN10 calculated from the averaged responses $(\mathrm{N}=2$, $\mathrm{n}=10$; means). Dashed lines represent the responses of the single individuals. B: Relative spike numbers of TN1 (brain recordings; $\mathrm{N}=15-16$; means and SE). Blue colouration - carrier frequency of the male song $(16 \mathrm{kHz})$ and red colouration - carrier of the female reply $(28 \mathrm{kHz})$.

The shortest latency of the second, tonic IPSP is found for $28 \mathrm{kHz}$ at $80 \mathrm{~dB}$ SPL with $47.7 \pm 3.9 \mathrm{~ms}(\mathrm{~N}=2, \mathrm{n}=9$; mean and $\mathrm{SD})$. This suggests an interneuron within the brain to be the candidate of the inhibitory input. In Figure 91 the relative amplitudes of the IPSP measured from the standard stimuli are displayed (dashed lines represent the single responses for 16 and $28 \mathrm{kHz}$ ).

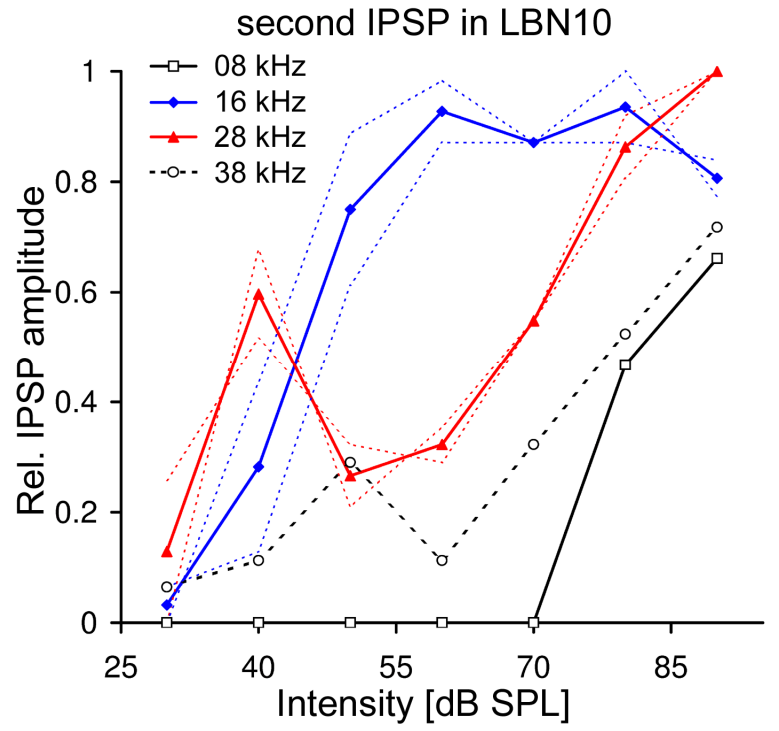

Figure 91: Relative amplitude of the IPSP in LBN10 calculated from the averaged responses $(\mathrm{N}=2, \mathrm{n}=10$; means). Dashed lines represent the responses of the single individuals. Blue colouration - carrier frequency of the male song $(16 \mathrm{kHz})$ and red colouration - carrier of the female reply $(28 \mathrm{kHz})$.

A first strong inhibition can be found for $28 \mathrm{kHz}$ at $40 \mathrm{~dB}$ SPL. The amplitude lowers between 50 and $70 \mathrm{~dB}$ SPL and strongly increases to 80 and $90 \mathrm{~dB}$ SPL. For $16 \mathrm{kHz}$ the amplitude of the IPSP increases steeply at low intensities and saturates 
above $50 \mathrm{~dB}$ SPL. Stimuli with 8 and $38 \mathrm{kHz}$, on the other hand, only evoke a strong IPSP at the highest intensities tested. Similar characteristics were also found in the IPSP amplitudes of LBN4 (see Figure 59A). The IPSPs in LBN4 may be provoked by LBN3 but the spike latencies of LBN3 are too short (26.8 $\pm 2.2 \mathrm{~ms}$ - see chapter 3.2.3) to be directly responsible of the inhibitory input to LBN10. 


\section{Discussion}

The accurate discrimination of conspecific from heterospecific acoustic signals is a demanding task that has to be fulfilled by the sense organ and the neuronal network within the CNS. A correct recognition, for example, alleviates mate finding and becomes crucial for the avoidance of predators (Nolan \& Hoy 1986). In the present study, the potential neuronal mechanisms behind the recognition process for the conspecific song have been examined in the brain of the southern-european bush cricket Ancistrura nigrovittata. Via intracellular recordings of single nerve cells possible candidates were detected, which may be responsible for male song or female reply detection. The response characteristics of five types of intersegmental neurons, which are already known from thoracic recordings in the past, were compared to those from recordings performed in the axonal portions of the same neurons in the brain. As expected, great similarity was observed in their spike activity to various kinds of acoustic stimulation. However, also differences were found that, in case of TN1, revealed a secondary auditory input in the brain. One additional ascending neuron was recorded (ANx), which has not been found in thoracic recordings. Several local auditory brain neurons, described for the first time in a bush cricket, were tested with special emphasis on their processing of the conspecific carrier frequencies and the temporal parameters important for recognition.

The following discussion will address (i) potential causes for the response differences that occur between intracellular recordings in dendritic and axonal portions of a neuron, (ii) the possible mechanism behind TN1's secondary graded input and its influence on the spike amplitude, (iii) the function of ascending interneurons and especially local brain neurons in the process of conspecific song recognition, (iv) redundant mechanisms found in the thorax and also in the brain, and (v) a comparison to auditory brain neurons of other species.

\subsection{Effects of cell penetration}

A neuron's excitability can differ in various portions along the nerve cells membrane (Furshpan \& Furukawa 1962; Tauc 1962), which correlates with an uneven distribution of voltage gated sodium channels. Highest density of sodium channels are reported to be located at the beginning of the axon (Wollner \& Catterall 
1986; Palmer \& Stuart 2006), the spike initiation zone. Also dendrites may exhibit certain "hot spots" that show an increased number of sodium channels with the possibility of spike generation (Spencer \& Kandel 1961; Haydon \& Winlow 1982). During intracellular recordings a neuron's membrane is penetrated by a sharp glass electrode, which inevitably causes cell injury. This sometimes is accompanied by an influx of extracellular fluid into the cell and thus, a depolarisation of the cell's membrane potential. Depending on the site of impalement and therewith the number of sodium channels in that location, the depolarisation may then cause spike generation. Various authors reported spontaneous activity that occurred with impaling a nerve cell (Fuster et al. 1965; Purves \& Sakmann 1974; Wohlers \& Huber 1978). This penetration dependent spontaneous activity was also seen in LBN4 of this study. During the search for local auditory neurons in the lateral protocerebrum, also intracellular recordings of interneurons were made that receive their major dendritic input in the prothoracic ganglion and exhibit an axon ascending to the brain. These data of spike activity could then be compared with data of the same neurons collected previously in the thorax. Thus, a comparison can be drawn between recordings with cell penetration sites close and far away from the spike initiation zone.

The overall response characteristics of the ascending neurons to the various stimuli tested in this study were, in most cases, very similar to the data obtained from dendritic portions and hence a reliable criterion for classification. In brain recordings the response of AN3, for example, showed the highest degree of accordance with the response characteristics described from thoracic recordings in all acoustic programs tested. The number of spikes elicited to stimuli at various frequencies, intensities and temporal variations were alike with virtually no variability. Although it has been pointed out that spontaneous activity, which has been frequently observed in AN3, may be due to depolarisation by the penetration (since also individuals without spontaneous activity have been recorded - Stumpner \& Molina 2006), various levels of spontaneous activity have been observed in the brain recordings. Therefore it is most likely, that different levels of spontaneous activity may be common in AN3 and not due to cell injury. On the other hand, the large difference in spike latency for low intensity stimulation between both recording-sites (see response at $30 \mathrm{~dB}$ SPL in Figure 15) might be an indication for a slight depolarisation by penetration in the thorax. During stimuli outside the range of best response the graded potentials in the dendritic region may slowly summate until reaching the spiking threshold. This process may be accelerated with depolarisation occurring during the impalement. 
Alternatively, this increased difference in latency may only be due to spontaneous activity. With decreasing stimulus intensity the latency of primary afferents will increase (for invertebrates: Mörchen et al. 1978; Rheinlaender \& Mörchen 1979; Oshinsky \& Hoy 2002). This long latency response will subsequently lead to longer latencies in first order interneurons (such as AN3) for low stimulus intensities. Since spike responses were evaluated in a definite time window for all intensities (see chapter 2.5), spontaneous spikes occurring earlier than the onset of stimulus evoked responses, would lead to a shorter mean latency value especially at low intensities. However, spontaneous activity does probably not account for the latency difference. Indications for that are found in the decreasing spike frequencies in brain recordings when compared to the thoracic data (data not shown in the results). When depolarisation by the penetration causes a change of the resting potential to more positive values, also an increased spike frequency can be expected during stimulation. A decrease of spike frequency can e.g. be found in AN1 for $16 \mathrm{kHz}$ at very low and very high intensities, where markedly longer spike latencies occur (see 30 and $90 \mathrm{~dB}$ SPL for $16 \mathrm{kHz}$ in Figure 9). Nevertheless, the response properties of AN3 obtained in the brain confirmed the data obtained in the thorax to the greatest extent among all the interneurons recorded in this study.

In AN1 and AN2 similar response characteristics were found for standard stimuli in a comparison of brain and thoracic recordings. The quantity of spike number mostly agrees for all intensities at a given carrier frequency. At high intensities (90 dB $\mathrm{SPL}$ ), though, one can see an increased spike number in the thoracic recording site. For the mean responses of AN1 this becomes evident for all frequencies tested and is also seen for the single responses shown for $16 \mathrm{kHz}$ (see Figure $6 \mathrm{~B}, \mathrm{C}$ ). In the case of AN2, this only seems to affect the activity at ultrasonic frequencies. For stimulations with varying temporal parameters this becomes even more obvious for the responses of AN1, AN2 and TN1. This clear difference in spike number is strong evidence for depolarisation by the penetration in the dendritic recording site. The effect is basically similar to a depolarising current applied by iontophoresis (e.g. during the injection of neurobiotin). Current induction also leads to an increased spike response due to the change of the neuron's resting potential to more positive values (e.g. Levine \& Murphey 1980; Hedwig 2000; own observations). However, one might assume that the spike number, when affected by depolarisation due to the penetration, is (more or less) uniformly shifted to higher values independent of the carrier frequency or temporal pattern of the stimulus and without impact on the overall response characteristics. This was not observed for pulse durations of $2 \mathrm{~ms}$ in 
AN1 (see Figure 8B). Here, the response in brain recordings exhibits a minimum, whereas thoracic recordings do not show a pronounced difference in AN1's response to any pulse duration tested. In AN2's response to different pulse/pause patterns a mostly uniform increase of spike number can be observed in thoracic recordings with the exception of a weaker response at $2 \mathrm{~ms}$ pulse duration and $61 \mathrm{~ms}$ pause duration (see Figure 12A, B). A possible explanation for this may be that depolarisation by the penetration may have a stronger influence on the spiking activity when the actual response of a neuron is already weak. This suggests that $2 \mathrm{~ms}$ pulse duration elicits only a minor response in AN1. During depolarisation an accelerated summation of the graded potential (see above) may only occur at weak responses. The same may also account for the spike number of AN2 during stimulation with varying pulse/pause duration. For comparatively weak responses spike number is increased in the thoracic recording, whereas this difference in response disappears when AN2 responds best. Another possible cause for the disproportionate increase in spike number due to the penetration in thoracic recordings may be the influence of the depolarisation onto the inhibition from a presynaptic interneuron. AN2 of A. nigrovittata gets frequency dependent inhibition (Stumpner 2001) that strongly affects the response during stimulation with short pause durations (A. Stumpner unpublished observations). Preliminary experiments with picrotoxin (PTX) show that the inhibition can be removed and the spiking response of AN2 for short pause durations is as strong as found for $61 \mathrm{~ms}$ pause duration. It might therefore be that depolarisation by penetration increases the probability of spiking especially when compound potentials are present. This may also account for the increased spiking response of $\mathrm{AN} 1$ at $90 \mathrm{~dB}$ SPL during the presentation of standard stimuli in the thoracic recordings. At these intensities also the inhibitory influence of a presynaptic neuron was observed (Stumpner 1997, 1998). The effects of depolarisation by the penetration may also contribute to the differences in the response characteristics of TN1 observed during the presentation of standard stimuli between thoracic and brain recordings. TN1 also gets inhibitory input that is strongest for low carrier frequencies and for contralateral stimulation (Stumpner 1999a, 2001). At low intensities (40 dB $\mathrm{SPL}$ ) the contralateral inhibition might not yet affect the spiking response of TN1 for ipsilateral stimulation (note the similar spike numbers in thoracic and brain recordings in Figure 19B). With increasing intensities also an increasing inhibition of response occurs in TN1. With depolarisation by the penetration in thoracic recordings, the effects of the increasing IPSP (especially at medium intensities between 50 and $70 \mathrm{~dB}$ SPL) may be reduced, which then contributes to the differences in the 
response characteristics to the brain recordings (apart from a high intraindividual variability). In AN5 also differences of the response to $28 \mathrm{kHz}$ stimuli at low intensities can be observed between both recording sites (see Figure 16B). These differences can be most likely explained by the strong penetration sensitivity of AN5 (which is also true for AN1 of crickets and AN4 in Chorthippus biguttulus; A. Stumpner personal communication). Often, AN5 stopped spiking due to the injury of penetration when intracellularly recorded in the thorax (which is not the case when recorded in the axon). A slight change of response characteristics may therefore be due to the sensitivity of penetration.

Additionally, also the preparation of the animal might contribute to the differences between the data obtained in the thorax and the brain. For thoracic recordings, one needs to expose the prothoracic ganglion and during the search for sound activated neurons within the auditory neuropil, neurons might have gotten injured that are presynaptic to the auditory neuron of interest. Thus, an increased input of excitation or inhibition may occur. In the brain preparation, this is not the case for neurons with an ascending axon and dendritic arborisations in the thorax. Therefore, a more reliable description of the output of a first order interneuron can be achieved by axonal recordings in the brain. Nevertheless, only intracellular recordings in the dendritic region can explore the complex nature of excitatory and inhibitory potentials.

\subsection{Graded potentials in TN1}

The general principle of information coding by a spike generating nerve cell is conversion of graded potentials in the dendritic region into all-or-none action potentials in the axon. Thus, the information of a neuron's output lies in the timing and rate of the action potentials that is passed on to a following neuron via its synaptic release. The amount of transmitter released at the presynaptic site mostly depends on the number and frequency of spikes elicited. Due to the all-or-none nature of action potentials the amplitude of single spikes is widely similar and causes a defined change in membrane potential and therefore the influx of a specific amount of calcium (Kandel \& Siegelbaum 2000). In the past, extensive knowledge has been gathered about how these defined amounts of transmitter release may be altered in the proximity of the presynapse. A common mechanism of presynaptic gain control is facilitation and depression mediated by molecular signalling cascades (Kusano \& 
Landau 1975; Byrne \& Kandel 1996; Gersdorff et al. 1997; Antonov et al. 2003). Here, changes in transmitter release are regulated indirectly and mostly occur over more or less long periods of time.

In axonal intracellular recordings of TN1 graded potentials can be observed that most likely originate from the activity of AN3 (probably via an intercalated interneuron). The occurrence of the graded potential is transient and contextdependent (dependent on AN3's response to the carrier frequency, intensity and temporal parameters), which, therefore, is neither due to a "slow" presynaptic facilitation nor depression that depends on intracellular signalling cascades. More likely, an involvement of ionotropic receptors that directly gate the influx of a certain ion at the presynaptic site (Siegelbaum \& Koester 2000) will cause such transient and contextual changes of the resting potential in the nerve cell's terminal. A basically similar mechanism is found in the presynaptic inhibition of afferents, also known as "primary afferent depolarisation" (PAD) (for review see Clarac \& Cattaert 1996; Rudomin \& Schmidt 1999). PADs are GABA-mediated changes in the chloride conductance in the axonal terminals of the target neuron. Thus, an extrusion of $\mathrm{Cl}^{-}$ may cause a slight depolarisation that has its reversal potential close to the equilibrium potential of chloride, which is typically about $4-20 \mathrm{mV}$ more positive than the resting membrane potential (depending on the nerve cell and organism Kennedy et al. 1980; Edwards 1990; Burrows \& Laurent 1993). An action potential, occurring simultaneously to a PAD, will exceed the reversal potential of chloride and thereby cause an influx of $\mathrm{Cl}^{-}$ions, which causes a decrease in spike amplitude. This mechanism of spike amplitude reduction (presynaptic inhibition) is not exclusively restricted to sensory afferents but has also been shown for interneurons in the central nervous system of the leech (Nicholls \& Wallace 1978). PADs mostly occur directly at the presynapse but can also be found in some distance to the synapse. The latter requires passive propagation in the axon terminal for a persistent reduction of the action potential's amplitude (Cattaert et al. 1994; Cattaert \& El Manira 1999). Strong indications for a passive spike propagation in (at least parts of) TN1's axon were found in this study. The graded potential increased in amplitude by injection of negative current with the possibility of exceeding the amplitude of a preceding action potential without eliciting additional spikes (Figure 25Bd). Contrary to the net effect of PADs, an action potential occurring simultaneously with a graded potential is increased in amplitude (and not decreased - see Figure 27). This summating effect reveals the excitatory nature of the graded potentials found in TN1's axon and 
therefore, a change of chloride conductance seems to be unlikely. Rather ions with more positive equilibrium potentials, such as sodium or calcium, may be involved.

Recent work on hippocampal mossy fibres of rats and layer 5 pyramidal cells of ferrets showed, that a subthreshold graded potential and a simultaneous action potential cause a greater excitatory response in the postsynaptic neuron than a single spike alone (Alle \& Geiger 2006; Shu et al. 2006). The graded potential by itself (in the absence of a spike) does not seem to be sufficient to trigger transmitter release, but indications were found that these potentials may increase the presynaptic calcium entry, which then leads to an increased transmitter release during the occurrence of an action potential (see Clark \& Häusser 2006 and Alle \& Geiger 2008 for a recapitulation of both studies). The graded potentials found in these studies, however, are generated close to the soma and propagate passively (whereby spikes are actively propagated) along the axon with a maximum range of about $1 \mathrm{~mm}$ (Alle \& Geiger 2008). In TN1, the axonal output site is too far away from the dendritic input site (ca. $4.2 \mathrm{~mm}$ ) to allow passive propagation of a subthreshold graded potential. Furthermore, the graded delayed potentials to acoustic stimulation were not reported from studies carried out in the dendritic portion of TN1 (Stumpner 2001, 1999a; Stumpner \& Molina 2006). Therefore, the transient changes of membrane potential in TN1's axon are generated locally within the brain. The findings cited above show that graded potentials, independent of being inhibitory (Clarac \& Cattaert 1996) or excitatory (Shapiro et al. 1980; Alle \& Geiger 2008), are able to alter either the spike amplitude or the resting calcium level in the presynapse or both, which causes a significant change in the membrane potential of the postsynaptic neuron. This makes it conceivable, that also the contextual change of spike amplitude in TN1 (see Figure 27) will have an effect on the postsynaptic nerve cell by an increased transmitter release. To my knowledge a similar mechanism, like the (reinforced) gain modulation found in TN1, has not been published for any other system yet.

The extent of the summating effect of spikes and graded potentials, however, differed in the various preparations of TN1, which may be due to three possible reasons. First, the exact site of axonal penetration varied in the different preparations and therefore, most likely also the distance to the input site of the graded potential. Since the graded potentials may also propagate antidromically, a reduced amplitude in comparison to a spike amplitude may be observed (and therewith the effect of summation - see Clarac \& Cattaert 1996). Second, injection of positive current slightly decreases the amplitude of a graded potential (see Figure 25B). During 
penetration, the axon of TN1 may have been depolarised, which caused an additional reduction of the graded potential's amplitude, besides a possibly far distance to the input site. Third, the modulatory effect of the graded potential on the amplitude of a spike may not compulsorily affect all axonal arborisations of TN1. If the input site of the graded potential is situated, for example, anterior to the first major branching point of TN1's axon (see Figure 18), then, a summating effect will only or mainly be observed in a specific branch (or a few branches). According to that, no summation may then be seen in the unaffected branch and the amplitude of the graded potential may be strongly decreased due to the distance of the input site. In the bush cricket Mecopoda elongata this "selective" modulation of single branches has been shown for auditory afferents (Hedwig \& Baden 2009). Here, presynaptic inhibition modulates the output of single branches of one sensory neuron, which might have an influence of the tonotopic presentation of carrier frequency within the prothoracic ganglion. In the visual system of the fly, a similar mechanism can be found, where single inputs of HSE cells ${ }^{1}$ or VS cells ${ }^{2}$ (1: equatorial horizontal system cell; 2: vertical system cell) are remarkably restricted to local areas of the dendritic branches (Borst \& Egelhaaf 1992; Borst \& Haag 2002).

\subsection{Possible function of the identified auditory brain neurons}

The involvement of the brain in the process of song recognition is proposed by several authors (e.g. Schildberger 1984a; Römer \& Seikowski 1985; Atkins et al. 1988) and was also experimentally shown for grasshoppers and crickets (Ronacher et al. 1986; Bauer \& Helversen 1987, Pires \& Hoy 1992). Models have been proposed that may have the ability to explain some of the neuronal processes in song recognition. Whereas frequency spectra may be of minor importance for the evaluation of conspecific signals, the recognition of the temporal pattern seems to be crucial in nearly all species (Fonseca et al. 2000; Pollack 2000). Two models, for example, suggested a coincidence detector, which compared the acoustic information with an internal template of the conspecific song (cross correlation - Hoy \& Paul 1973, Hennig 2003) or with a secondary, delayed pathway of the auditory information (auto correlation - Reiss 1964; Weber \& Thorson 1989). A necessary prerequisite for the latter is a secondary pathway that exhibits an internal delay of the pulse pattern by (at least) one period. If the undelayed pathway then matches the pulse pattern of the delayed pathway, a maximum excitation can be achieved. Both 
models, however, have been partly rejected in the context of conspecific song recognition (Pollack \& Hoy 1979; Helversen \& Helversen 1998; Bush \& Schul 2006). Another model for a narrow behavioural response proposes a band-pass filter, which originates from combining a high- and a low-pass. This kind of filter properties has been shown for single interneurons in the brain of Gryllus bimaculatus (Schildberger 1984a). Evidence for a band-pass filter does not exclude deviant filter mechanisms that may be established in different species (for a review see Hennig et al. 2004). An additional, more recent model for recognition bases on oscillatory membrane properties, which allow pulse rate recognition by a correct resonance (pulse rhythm of the conspecific song) in a single interneuron (Bush \& Schul 2006). In another model a sufficient analysis may already occur on the thoracic level by the instantaneous discharge rate of ON1 (Nabatiyan et al. 2003; for a review see: Hedwig 2006).

Processing of conspecific carrier frequency. In the species Ancistrura nigrovittata, behavioural experiments showed that the correct temporal parameters of the male song and, quite unusually, also the sex specific carrier frequency (which is mostly of minor importance - see above) lead to conspecific recognition (Dobler et al. 1994a, b). Previous data of interneurons that receive major input in the prothoracic ganglion and exhibit an ascending axon to the brain suggested a classification of three different "channels" for processing of carrier frequency (Stumpner \& Molina 2006).

The first channel consists of AN1 and AN2, which seem to be critically involved in male song processing due to their low thresholds for sonic frequencies. The tuning to medium and high sonic frequencies with prominent optimum type intensity response functions is especially pronounced in AN1, whereby ultrasonic frequencies evoke the lowest activity which is partially due to inhibition (Stumpner 1997). An involvement in positive phonotaxis to the male calling song has been shown for the homologous AN1 in crickets (Schildberger \& Hörner 1988) and also seems to be the case in A. nigrovittata. AN2, on the other hand, also showed strong bursts to the female reply when recorded in the brain (not reported from thoracic recordings - see Stumpner \& Molina 2006). According to these findings, AN2 may also partly belong to the second category - the ultrasound channel.

The ultrasound channel includes neurons like AN4 (which has not been recorded in the brain), AN5 and TN1. Among these neurons, AN5 responds most specific to the female reply, which was also shown by the data obtained in the brain. 
If one compares the responses of AN5 (Figure 17B) and TN1 (Figure 24) to varying female reply carrier frequencies, a reduced response, to the highest ultrasonic frequencies tested, can only be found in AN5, which results in a narrower tuning to the female carrier frequency of $28 \mathrm{kHz}$. However, both neurons showed little or no response to the male song frequency (which is, in the case of TN1, contrary to the finding obtained in the thorax - see Stumpner 1999a, b; Stumpner \& Molina 2006). Due to the carrier frequency tuning possibly one additional neuron, namely ANx (that has not been recorded in the thorax), may also belong to the ultrasound channel, although nothing is known about the position of the dendritic input site. Its low thresholds to all ultrasonic frequencies tested (see Figure 30B \& C) may implicate a possible involvement in bat avoidance behaviour, in addition to TN1 (Libersat \& Hoy 1991; Stumpner \& Molina 2006).

The third channel involves AN3, a neuron that responds to nearly every sound at any carrier frequency that is presented. It is supposed to act as a "reference channel" that is active during the presence of an acoustic signal (Stumpner \& Molina 2006). Ascending vibratory neurons show no response to sound stimuli at low intensities. Therefore, it is unlikely that these neurons are involved in the processing of intraspecific long distance communication signals. Only in the close proximity of the duetting partners, where vibrational communication signals can be perceived, vibratory neurons may be relevant for the process of recognition (Heller \& Helversen 1986).

For the local brain neurons of $A$. nigrovittata recorded in this study, the same categorisation can be applied due to their responses to different carrier frequencies. The first channel - the sonic sound channel - comprises LBN1, LBN4, LBN6 and LBN7. Strong evidence has been presented that LBN1 is directly connected to AN1, which is also seen in the nearly identical intensity response characteristics to standard stimuli. The response threshold to $16 \mathrm{kHz}$ stimuli is significantly shifted to higher intensities (in comparison to AN1 responses), which shows a higher degree of correlation to the female behaviour than that found in AN1 (Figure 44A). The spike activity of LBN1 is narrowly tuned to the male song frequency at low intensities and ultrasound elicits nearly no responses. The latter is mainly due to the low input by AN1, the spike frequency filter of LBN1 and the frequency specific inhibition seen in LBN1. From the close correlation of LBN1's spike activity to the female behaviour, LBN1 seems to be the decisive neuron for the detection of the male song carrier frequency. 
The response of LBN4 is also tuned to sonic frequencies. This is seen, despite strong spontaneous activity and inhibition, at the initial excitatory potential at 8 and $16 \mathrm{kHz}$ for low intensities (Figure 58). Possibly, AN1 may cause the excitatory input to LBN4, since the low threshold EPSPs at $16 \mathrm{kHz}$ have shortest latencies of $19.3 \mathrm{~ms}$ at $50 \mathrm{~dB}$ SPL, the intensity at which AN1 also responds best.

LBN6 is a neuron that responds best to lower sonic frequencies than those found in the male calling song. A possible function of such a neuron type may be seen in the low frequency inhibition in other interneurons resulting in a narrowly tuning, for example, to the male song frequency. Although no brain neuron has been recorded that receives such frequency inhibition, a similar type of low frequency inhibition has been found on an earlier stage of auditory processing, in the dendritic region of AN1 in the thorax (Stumpner 1997, 1998).

LBN7 shows very similar response characteristics as found in AN2 and a direct connection between the two neurons is likely (Figure 70B, C). Lowest thresholds are also found for $16 \mathrm{kHz}$ during presentation of standard stimuli. Similar to AN2, also LBN7 shows a strong bursting response to the female reply at $60 \mathrm{~dB} S P L$, which therefore partly permits a categorisation of LBN7 to the ultrasonic channel.

The ultrasonic channel consists of cLBN5, cLBN8 and LBN9. Intracellular recordings of the interneuron CLBN5 revealed strongest responses to all ultrasonic frequencies tested without a specific tuning to the female carrier frequency (Figure 64C). Thus, cLBN5 may be involved in bat detection and/or may (also) be responsible for ultrasonic frequency inhibition in other neurons.

The responses of CLBN8, on the other hand, only occur to a narrow range of ultrasonic frequencies at low intensities (Figure 78B \& 82). At $40 \mathrm{~dB}$ SPL it shows strongest responses at $28 \mathrm{kHz}$ to standard stimuli, whereas the female reply at $60 \mathrm{~dB}$ SPL reveals best responses at $24 \mathrm{kHz} .24 \mathrm{kHz}$ is also represented in the frequency spectra of the female reply (Dobler et al. 1994b) and therefore, a response to this carrier frequency does not exclude a potential involvement in processing of the female reply frequency. Interestingly, the response of cLBN8 to $28 \mathrm{kHz}$ at $40 \mathrm{~dB} \mathrm{SPL}$ only (during standard stimulation), is very similar to the response of $\mathrm{ANx}$ (compare Figure 78B \& 30A). A closer examination of the graded potential, however, reveals a correlation to the spike activity of TN1 (Figure 80). Thus, similar response characteristics can be achieved by the presynaptic input of different neurons with different response characteristics. The narrow intensity dependence of cLBN8's response found at $28 \mathrm{kHz}$ during standard stimulation would suggest a good response, when a female reply is heard by a male at a large distance. According to 
this, cLBN8 may function as a detector for conspecific females that are not in the close proximity of the singing male. Due to the axonal arborisations this information from one ear may then be integrated in both auditory neuropils of the brain (see Figure 77).

When presenting an artificial duet, a response of LBN9 can only be seen at a narrow carrier frequency range that correlates with the female spectrum (Figure 85A, C). At medium and high intensities also an initial EPSP can be observed to the beginning of the syllable series of a male calling song, which is followed by inhibition. This inhibition is strongest at ultrasonic frequencies and high stimulus intensities (Figure 86). These response properties may imply a possible function of LBN9 as a long distance female detector as found in CLBN8.

In the brain also neurons are found that can be classified to the third channel (reference channel). This channel consists of LBN2, LBN10 and partly of LBN3. LBN2 shows highest spiking activity to an artificial duet (Figure 46A). When presenting an artificial male song at the four standard frequencies $(8,16,28$ and $38 \mathrm{kHz}$ ), it responds best to the male song frequency at low and medium intensities (Figure 46C). An evaluation of LBN2's response to varying female reply frequencies in an artificial duet shows highest spiking activity to $28 \mathrm{kHz}$ only. Also at $16 \mathrm{kHz}$ a slightly increased response can be observed. Contrary to AN3, which indicates the presence of any acoustic event, LBN2 may indicate the presence of any conspecific communication that occurs in the environment with a consistently lower response at other than the conspecific carrier frequencies. Hence, in the case of LBN2, the reference channel properties are narrowed to a conspecific duet. Additionally, LBN2 receives inhibition in the ultrasonic frequency range. This may indicate that this reference channel gets inhibited by predatory signals such as bat calls.

LBN10 is a neuron that exhibits high spontaneous activity and gets inhibited by a wide range of carrier frequencies (Figure 89) and thus, also by an artificial duet (Figure 88). Spikes occurring during the IPSP of LBN10 indicate that the penetration of the neuron was performed at some distance to the spike initiating zone. The spikes must have been travelling antidromically to the penetration spot, which is situated close to the input of the IPSP. Thus, the large IPSP as seen in Figure 88 might be decayed when entering the zone where action potentials are initiated. A possible function of LBN10 may be the constant inhibition of a postsynaptic neuron by its spontaneous activity. During an acoustic event this inhibition may stop and allow spiking in the postsynaptic neuron. According to this, a certain channel may be unblocked that is only active during airborne sounds. LBN10 may therefore have a 
similar effect on postsynaptic neurons as AN3, though with an inverse mode of operation.

As mentioned above, LBN3 only partly functions as a reference channel. Strong indications were presented that LBN3 receives its excitatory input from AN3 (Figure 53 \& 54). Hence, a response to the male calling song and to the female reply can be found (Figure 52A). An evaluation of LBN3's response to the female reply with varying frequencies reveals a strong response to the female carrier frequency only (Figure 52C), which then allows a partly categorisation in the ultrasound channel. The most likely function of LBN3 is the "switch" of excitatory input from AN3 into inhibitory input to LBN4.

Processing of conspecific song pattern. Several possible candidates have been suggested by Stumpner \& Molina (2006) to be involved in the temporal processing of the male syllable series from the data obtained of the intersegmental neurons in the thorax. A possible basis for band-pass filtering of pause duration in a behaviourally relevant context may be achieved by the increasing response strength of AN2 and AN4 to increasing pause durations, in combination with the decreasing response strength of AN3. Furthermore, band-pass characteristics can also be found in the activity of AN1 or TN1 (Stumpner \& Molina 2006). From the data obtained in the axon of the ascending neurons, the response properties to a variation in pause duration as recorded in the thorax were confirmed. Contrary to this, the data gathered in the axonal portions of AN1 and AN2 show a close correlation to the female behaviour in the response to short/long pulse duration, which has not been found in thoracic recordings (Stumpner \& Molina 2006). The response strength of AN1 recorded in the axon decreases strongly for the shortest pulse durations tested, similarly as the female phonotactic response (Figure 8B right; Dobler et al. 1994a). For pulse duration longer than $7 \mathrm{~ms}$ the female response is reduced, which is paralleled by the lowering of response strength in AN2 (Figure 12B right). Thus, the narrow tuning of the female behaviour to the male's syllable duration may be achieved by the combined evaluation of AN1's and AN2's response - the interneurons that also respond best to the male song frequency at low intensities (see above). A "low- and high-pass filter" for pulse duration may therefore be established on the thoracic level as proposed by Schildberger (1984a).

AN5 recorded in the brain shows a stronger response to the shortest pulse durations tested, although the overall spiking activity was very low (Figure 17A). A similar increase in response was found in the axonal recordings of TN1 (Figure 23B), 
which is a further indication (in addition to the ultrasonic frequency processing) for a potential relevance in the recognition of the short duration female reply to the conspecific song (Heller \& Helversen 1986) or the short duration of a echolocation sound emitted by a bat (Simmons 1989). The graded potentials found in the axonal branches of TN1 most likely originate from AN3 (see previous chapter and chapter 3.1.5). Action potentials occurring simultaneously to the graded potentials are increased in amplitude and therefore, may potentially increase the synaptic transmitter release and the excitement of the postsynaptic neuron. Since TN1 shows highest spike activity to $28 \mathrm{kHz}$ stimuli (at low intensities; Figure 19B) and the graded potential is highest with $16 \mathrm{kHz}$ stimuli (at low and medium intensities; Figure 26B), the possible context may then be as follows: If a male hears its own song (or possibly the song of a different male) an EPSP will be provoked in TN1. In dependence of the distance of the duetting partners the spikes to the female reply in the male's TN1 will be pronounced, when they occur in a certain time-window corresponding to the duration of the graded potential to the own trigger syllable. Therefore, the strength of excitation of TN1's postsynaptic neuron in the brain of a male will be correlated with the distance to the responding female (see Figure 28B). The behavioural responses of males also are restricted to an internal time window in which a conspecific female answer can be recognised by the male (see Heller \& Helversen 1986; Zimmermann et al. 1989; preliminary results of Pierre Gras for A. nigrovittata - orange box in Figure 28B). The responses of AN3 were similar in both recording sites and do not show a clear correlation to the female behaviour (Stumpner \& Molina 2006). The magnitude of ANx's spike activity shows a close correlation to the female behaviour, when presenting male song models consisting of white noise and varying pause durations (Figure 31). Due to the higher sensitivity for ultrasound (see above) and the high response to short pulse durations only (Figure 32), it is more likely that ANx rather is involved in bat detection than in the processing of the male song.

Nearly all of the local auditory brain neurons presented were tested for their temporal processing, which therefore allows to hypothesise about a possible relevance in the recognition process of the conspecific song. In LBN7, the strongly decreasing responses to longer pulse durations, that closely resemble the female behaviour, are also found in its presumed presynaptic neuron - AN2 (the same applies for the stimuli with variations in pause duration; see Figure $72 \&$ 73). These findings for LBN7 may also apply for LBN1, which receives direct input from AN1. Therefore, a low response of AN1 to short pulse durations may also be found in LBN1 (preliminary data supports this hypothesis). Thus, also on the second level of 
auditory processing (the lateral protocerebrum of the brain) a high- and low-pass filter for pulse duration is established (see above). LBN7 additionally shows a secondary response with a long latency of approximate $600 \mathrm{~ms}$ subsequent to high intensity stimulation (lower intensities were not tested with a sufficient post-stimulus pause). This secondary response was strongest for $28 \mathrm{kHz}$ stimuli but was also seen at $16 \mathrm{kHz}$. Although Dobler et al. (1994a) reported that females did not respond to an artificial song with an interval between syllable series and trigger syllable being greater than $500 \mathrm{~ms}$, intervals of ca. $600 \mathrm{~ms}$ can be observed in a natural male calling song with a clear response of females (see Figure 5 Top in Heller \& Helversen 1986). A response property as found in LBN7 would explain, why motivated females reply to a male song at approximately the correct time, even if a trigger syllable is missing (Dobler et al.1994a). Such a long latency response has not been observed in AN2 (the presynaptic neuron) or in any other ascending neuron.

LBN2's low response to short pulse durations (which is due to inhibition - see Figure 51) correlates to the decreasing behavioural response of females (Figure 50B), whereas the spiking activity to varying pause durations does not show an obvious correlation to the specificity of the female behaviour. During a presentation of an artificial duet and varying female reply latencies, one can see the absence of response to the reply when the pause is short (Figure 48; as already reported for AN5 - Stumpner 1999b). Thus, LBN2 may additionally contribute to the male time window, in which female responses have to occur to be recognised by the male. LBN4 possibly gets excitatory input from AN1 and strong subsequent inhibition from LBN3. When presenting an artificial duet, one can see excitation to the beginning of the syllable series as well as to the trigger pulse. Thus, LBN4 marks the beginning and the end of a male verse (Figure 57A). A potential function for this kind of male song evaluation is not obvious, since the behavioural data clearly shows a dependence on pause duration between the end of the syllable series and the trigger syllable. The beginning of the verse in correlation to the trigger pulse, on the other hand, seems to be irrelevant (Dobler et al. 1994a).

From the frequency tuning of CLBN5 an involvement in bat detection is likely, which is also assisted by the strong response to short pulse durations (Figure 65B). For varying pause durations an optimum type characteristic is found in the spike activity of cLBN5 that has no obvious correlation to the behaviour. Apart from a possible function in bat detection, an inhibitory influence on other auditory interneuron may be possible, since ultrasonic frequency inhibition is frequently found in the auditory system of $A$. nigrovittata. The overall response of cLBN5 to different 
temporal pattern is very similar to ANx (see Figure $31,32 \& 65$ ). Thus, cLBN5 may receive input from $\mathrm{ANx}$ since also $\mathrm{ANx}$ axon arborises in the caudal lateral protocerebrum, where cLBN5 has its dendritic input-site (compare Figure 29 \& 63). LBN6 shows strong spiking activity to carrier frequencies much lower than $16 \mathrm{kHz}$. Additionally, the response to the varying temporal pattern is lowest, where the behaviourally response of females is highest (Figure 68). A likely function of LBN6 may therefore be an inhibitory influence on neurons that process the calling song of males. This inhibition would lead to a high-pass frequency filter and a low response to unattractive pulse/pause patterns. The spike activity of CLBN8 is strongest to the shortest pulse durations tested (Figure 81B), which may also be expected from the narrow tuning to $24 \mathrm{kHz}$ and therefore, the possible function in detecting the female reply. The spike number does not exceed 2 at maximum, which is most likely due to the delayed ultrasonic frequency inhibition with a remarkably long duration of about $200 \mathrm{~ms}$.

In summary, on the thoracic level a filter for the male song carrier frequency is achieved by AN1's response up to ca. $50 \mathrm{~dB}$ SPL and by the combined responses of AN1 and AN2 at higher intensities. Additionally, the combined responses of AN1 and AN2 may also be sufficient for the evaluation of the male song pulse duration. None of the described intersegmental neurons show a close correlation to the complete female behaviour in regards to the pause duration, although AN2 may contribute to the decreasing behavioural response at shorter pause durations. The female reply, on the other hand, may be processed by AN5 and/or TN1, whereas TN1 may additionally have its relevance in bat avoidance behaviour (as well as ANx). This potential importance for both, song recognition and bat avoidance has also been suggested for AN2 of crickets (Nolen \& Hoy 1986; Schildberger \& Hörner 1988). AN3 is always active whenever audible sounds are present, regardless from being of species specific origin or not. The processing of conspecific song is certainly not completed on the thoracic level and the information about certain song parameters is most likely distributed over several elements. The brain, therefore, may house the decisive elements for recognition of the conspecific partner. For the male carrier frequency, the responses of LBN1 show a closer correlation to the female behaviour than those of AN1. The combination of LBN1's and LBN7's responses may, therefore, "explain" the processing of the male song pulse duration (similarly to AN1 and AN2). In the possible inhibitory response of LBN6 a sufficient band-pass filter may be established, that correlates closely to the female behaviour to both, the male 
song pulse duration and pause duration (no other neuron showed a closer correlation to the behavioural data to varying pause durations). The detection of the trigger pulse of the male calling song may be emphasised by the secondary response seen in LBN7. Brain neurons that are possibly important for the detection of the female reply are cLBN8 and LBN9. The new properties of TN1 found in the brain may also indicate a functional relevance for female detection. For bat avoidance, most likely the responses of cLBN5 may be important, since highest spike activity can be found to every ultrasonic frequency tested without specific tuning to the female reply carrier frequency. LBN2 acts as a reference channel during conspecific signalling and may necessarily need to be active to elicit positive phonotaxis to a male's call or the female reply. Such a reference channel may also have its relevance in the localisation of a conspecific signal, if responses in neurons with strong contralateral inhibition are suppressed. The release of the short female reply, which followed the male trigger pulse with a constant latency of about $30 \mathrm{~ms}$ (see Heller \& Helversen 1986; Dobler et al. 1994a), most likely does not involve neuronal activity of the brain. In this study none of the recorded interneurons had sufficiently short latencies to directly elicit the female response. Since the relevant motorneurons are situated in the mesothoracic ganglion, it is assumed that the release is due to a fast acoustomotor reflex, where brain processes are not involved (Hennig 1989). In A. nigrovittata females, however, this fast reply also depends on processing of the male carrier frequency. Variations of the carrier frequency of the male trigger syllable (while keeping the syllable group at the male carrier frequency - $16 \mathrm{kHz}$ ), showed similar behavioural responses as found for varying frequencies of the whole calling song (Dobler et al. 1994b). This would imply a local mechanism being established already in the thorax (without direct involvement of the brain) that has the ability of a fast evaluation of the carrier frequency.

Each local auditory brain neuron presented in this study (except for LBN4 with $\mathrm{N}=1$ ) was recorded (and therefore is represented) in both sexes without obvious response differences observed. This was also reported previously for the intersegmental neurons of the prothorax (Stumpner 1997; A. Stumpner - personal communication). These findings may indicate that both sexes exhibit the same sets of auditory interneurons with similar response properties and thus, possibly similar recognition processes. The only difference may then exist in a sex specific connection to descending interneurons (command neurons), which activate different pre-motor networks in the mesothoracic ganglion and therewith different kinds of sounds that are produced (Hedwig 2000). 


\subsection{Redundancy in the coding of information}

As detailed before, the presented data of this study leads to the conclusion that LBN1 is a second order interneuron and is directly connected to AN1. This conclusion is based on similar shape of intensity response curves at standard stimulation and on corresponding spike/EPSP frequencies at the same stimuli (Figure 36A, B \& 39). It is also supported by a small difference (below $2 \mathrm{~ms}$ ) between latency of AN1 spikes and LBN1 EPSPs (Hennig 1988). The same holds true for the possible connection of AN2 and LBN7. The responses of LBN7 to standard stimuli, to the male song models with varying temporal pattern and to the female reply with variation in carrier frequency (during an artificial duet) is widely similar to the spike activity of AN2 (e.g. see Figure 70).

In addition to excitation, LBN1 and LBN7 receive clear inhibition, at similar stimuli, at which also AN1 and AN2 receive inhibition. In the case of LBN1, the inhibition likely does not come from an ascending cell but from another brain neuron (due to the long latencies). Prominent IPSPs can be observed during depolarisation with lowest thresholds at ultrasonic frequencies. In nearly all recordings indications of IPSPs were observable also without depolarising current, but these IPSPs mostly were expressed only as parts of compound potentials. The resulting effect is a further reduction of responses to ultrasonic frequencies than already found in AN1 (Figure 44B). In contrast, Rheinlaender (1975) concluded that the frequency selectivity of unidentified neurons in the brain of a bush cricket does not differ from that in the thorax. In LBN1, during recordings with a depolarising current a biphasic IPSP is clearly seen (Figure 41). The biphasic nature of the IPSP might either originate from the input of two different (yet unknown) interneurons in the brain (as well as AN1 obviously gets inhibited by two different thoracic interneurons, Stumpner 1997) or from one single brain interneuron that exhibits characteristics of a phasic On-Off-response. In the latter case, one can speculate which ascending interneuron might be involved in the inhibitory pathway to LBN1. An ascending interneuron producing an On-Off-response is not known. Therefore, a neuron with a tonic broad banded response to auditory stimuli with lowest thresholds in the low ultrasonic frequency range (and possibly in the high sonic range) would carry the necessary information, since phasic responses of neurons like TN1 would only mark the beginning of the stimulus without any information about its end. The only known candidate is ascending neuron 3 (AN3) which exhibits broad banded frequency selectivity (nearly identical to the hearing threshold; see Stumpner \& Molina 2006) 
and tonic response characteristics for copying the whole length of the stimulus. All other thoracic interneurons known, exhibit phasic (On-) responses or too high thresholds for the required frequency range (Stumpner 1999a; Stumpner \& Molina 2006).

Since the excitation concealed the beginnings of the IPSPs observed in LBN7, no latencies could be determined for the inhibition. Thus, it is unknown whether the inhibition originates from an ascending or a local cell. However, stimulation with standard stimuli contralateral to the side of penetration revealed a pronounced decrease in response when compared to the responses to ipsilateral stimuli (Figure 71). This indicates that the inhibition in LBN7 (at least partially) comes from the contralateral ear, which has also been shown for the inhibitory input in AN2 by ablation experiments when cutting the contralateral auditory nerve (respective to the side of penetration; A. Stumpner - unpublished).

The two examples described above show clear indications of redundant processing mechanisms established in serially connected neurons. Also in the other brain neurons (presented in this study) processing mechanisms are found (mainly frequency specific inhibition) that are already established on the first level of processing - the prothoracic ganglion. Why does an interneuron in the brain receive frequency specific (LBN1) or contralateral (LBN7) inhibition, when it's presynaptic interneuron (AN1 and respectively AN2) in the thorax already undergoes exactly the same filtering? For LBN1 an increased threshold for ultrasonic frequencies is shown, when comparing the lowest thresholds for subsequent stations of processing (Figure 44B). This is most likely is due to two factors: (i) a spike-frequency filtering and (ii) the just mentioned inhibition. Therefore, the exclusion of ultrasonic frequencies in the pathway of male song recognition is secured by a multiple stage inhibition in a redundant fashion. This kind of redundancy can be found in various neuronal networks and it seems to be a common strategy in filtering relevant information. For example, the corollary discharge interneuron (CDI) in the central nervous system (CNS) of the cricket Gryllus bimaculatus serves the function of preventing the auditory system from desensitisation by self-generated signals (Poulet \& Hedwig 2006). Inhibitory connections are made with primary afferents and several first order interneurons in the prothoracic ganglion as well. The extensive axonal arborisations of $\mathrm{CDI}$ in every ganglion (including the brain) of the CNS suggest additional inhibitory connections to local brain neurons involved with auditory processing. Thus, self-generated noise gets inhibited on multiple stages in the auditory processing chain and not at one particularly decisive step in this chain (e.g. 
at an early stage only). Another example of redundancy can be found in the contrast enhancing network in the retina of vertebrates (for a summary see Tessier-Lavigne 2000). Ganglion cells report contrasts of luminance in their receptive field to higher brain centres. Prior to this, the information about contrast gets enhanced by horizontal and amacrine cells in the outer and inner plexiform layer of the retina. Both types of neurons in the lateral pathway receive input of surrounding photoreceptors or bipolar cells to enhance the contrast in the centre-surround receptive field of the respective ganglion cell. The vertical pathway in the retina (photoreceptor - bipolar cell - ganglion cell), therefore, is influenced in a similar fashion at more than one stage to fulfil the task of contrast enhancement.

In conclusion, filtering of relevant conspecific signals experiences a (fine) tuning in a "step by step"-fashion with the use of redundant mechanisms. Thus, the filtering of important information (as e.g. male song carrier frequency) does not rely on the processing of a single neuron, but rather on the processing of multiple units connected in serial. The output of the neuron last in chain (e.g. for male song carrier frequency: potentially LBN1), therefore, is of particular importance for the processes of recognition. This secures the reliability of the whole system rather than being dependent on the filter processes of a single neuron. If the latter would apply, a narrow filter for male song carrier frequency would already be established on the level of a single sensory neuron in the ear that may sufficiently describe a behavioural tuning to the conspecific song (Ostrowski \& Stumpner submitted). For a grasshopper it has been demonstrated that the activity of single sensory neurons suffices to transport the relevant information on song structure (in this case a broadband song - Machens et al. 2003). However, producing behaviour on the basis of just one out of ca. 37 sensory neurons may simply be too risky in terms of reliability of information processing, even though insects exist, which in principal base their decision on an individual sensory neuron's activity (e.g. certain moths - Surlykke 1984). For bush crickets, however, this narrow tuning is lost due to the convergent input of more than one sensory neuron to first order interneurons (Stumpner 1997, 1998, 2002).

Not all processing mechanisms in the brain are redundant. One particularly novel characteristic deserves to be mentioned at this point. In local brain neurons excitatory and/or inhibitory graded potentials can be observed, which exhibit extremely slow decay time constants. In cLBN8 delayed IPSPs are found that have a total duration of approximately $200 \mathrm{~ms}$ to a $50 \mathrm{~ms}$ stimulus (Figure 83). Also in LBN9 as well as LBN10 IPSPs with very long durations are found, which are caused by the 
male song syllable series and have the ability to last until the male trigger pulse (350 ms later; Figure 85A \& 88). An even greater extreme is found in the EPSP of LBN9 elicited to a short female reply ( $3 \mathrm{~ms}$ pulse) that may last for several hundred milliseconds (Figure 87). For the omega neuron of the bush cricket Tettigonia viridissima also long lasting changes in membrane potential have been reported (Römer \& Krusch 2000). These long time constants (in the order of several seconds), however, can be ascribed to post-excitatory inhibition rather than IPSPS or EPSPS directly caused by auditory stimuli.

\subsection{Comparison to other brain neurons}

The morphological appearance of nearly all local brain neurons described in this study are unique among other auditory brain neurons found in ensiferan species (only crickets), since shape and arborisation pattern clearly differ from those published to date (Boyan 1980, 1981; Schildberger 1984a, b; Atkins et al. 1988; Brodfuehrer \& Hoy 1990). LBN2 comprises great similarity in the position of the soma and the major arborisations to BNC1a (brain neuron class 1a) reported by Schildberger (1984a). BNC1a is narrowly tuned to the male calling song frequency of Gryllus bimaculatus only (ca. $4-5 \mathrm{kHz}$ ). LBN2, on the other hand, shows a narrow tuning to the male's song and additional sensitivity to the female reply in the ultrasonic range (see chapter 3.2.2). Although different stimuli were used, the responses of both neurons show a good coupling to the stimuli with variations in the temporal parameter (even though LBN2 shows a slight decrease in response to shorter pulse durations and pause durations between 5-35 ms - Figure 50). Due to the criteria for categorisation suggested by Schildberger, BNC1a seems to be a second order interneuron, which is not the case for LBN2 with EPSP latencies of approximately $35 \mathrm{~ms}$ (for the IPSP in LBN2 a direct connection to ascending interneurons may be possible). However, too little information is given about individual BNC1 neurons of crickets to allow a closer comparison. The same is true for LBN-e1 (local brain neuron-excitatory 1) described by Brodfuehrer \& Hoy (1990) for the cricket Teleogryllus oceanicus, where little emphasis had been laid upon a detailed description of single interneurons. The morphology of LBN-e1 shows some similarity to CLBN5, with the caveat that nothing can be concluded about the soma position of LBN-e1. Both neurons exhibit high-pass carrier frequency processing in the ultrasonic range (LBN-e1 additionally in the high sonic range) and therefore, may 
have relevance in predator avoidance. Because of the absence of adequate criteria of homology (except partially for the morphology) to all brain neurons known in other species, for the presented brain neurons of this study a neutral terminology "local brain neuron" (based on Brodfuehrer \& Hoy 1990) was used.

If one classifies the overall spatial extension of the brain neurons described in this doctoral thesis using the criteria suggested by Schildberger (1984a), nearly all of the neurons would belong to brain neuron class 1 (BNC1) due to the overlap with arborisations of ascending neurons and/or the direct input by those (except for LBN6, where no staining and no graded potentials were available for analysis). This finding may indicate that the auditory organisation in the brain of bush crickets may differ from that of crickets, since no local brain neuron found in this study fulfils the criteria for belonging to BNC2 neurons. Furthermore, a spatial separation of low and high carrier frequency processing neurons, as suggested by Boyan (1980), was not found.

A narrow frequency tuning to the conspecific calling song in combination with an increased threshold as in LBN1 is also true for neurons of both classes described by Schildberger (BNC1 and BNC2). Except for the BNC1 and BNC2 neurons, all other neurons identified in the brain of crickets so far (e.g. Boyan 1980; Atkins et al. 1988; Brodfuehrer \& Hoy 1990) were not tuned to the male calling song but are most likely involved in processing of predator signals (or perhaps also courtship songs). In regards to the temporal processing, low-, high-, band-pass and band-suppressor neurons, as found in crickets (except for the band suppressor - Schildberger 1984a) and the auditory nuclei of frogs (for a summary see Gerhardt \& Huber 2002), are also found in A. nigrovittata (although only pulse/pause-durations were tested). High- and low-pass filter for certain temporal parameters, which closely correlate to the female behaviour, may be found in the responses of AN1, AN2 and LBN7 (and maybe in LBN1 due to the direct connection to AN1 - see above). A narrow band-pass filter for pause duration is found in cLBN5, whereby band-suppressor properties for varying pulse/pause durations is found in LBN6. 


\section{Summary}

In this doctoral thesis intracellular recordings of identified auditory neurons in the brain of a bush cricket are presented for the first time. Single cell recordings were carried out in the species Ancistrura nigrovittata (Brunner von Wattenwyl, 1878) with special emphasis on filtering properties for the conspecific song. The coding of carrier frequency, intensity and temporal pattern was examined in local brain neurons and in intersegmental neurons for a comparison with the behavioural responses to these parameters.

Spike activity of five types of ascending intersegmental neurons was acquired from the axonal terminals, which allowed a correlation to earlier results obtained from the dendrites. The response characteristics found in both recording sites are widely similar (closest in AN3), although differences have been observed. These are e.g.:

(i) filter properties in the spike activity of AN1 and AN2 to some pulse durations, which closely correlate to the female behaviour

(ii) graded potentials in TN1's axon with the capability to facilitate the amplitude of action potentials in a graded manner close to the presynaptic output site

(iii) a physiological and a morphological description in the brain, of a so far unknown intersegmental neuron (ANx) that processes ultrasound stimuli

A characterisation of ten types of local auditory brain neurons is given, whereby novel as well as known response properties with a surprisingly high degree of redundant filter mechanisms are found. These are e.g.:

(i) a spike frequency filter and a redundant frequency dependent inhibition in LBN1 resulting in a response that has a closer correlation to the female behaviour than found in the spiking of its presynaptic neuron (AN1)

(ii) the narrowly tuned activity of LBN2 to conspecific signals of both sexes representing a channel that indicates species specific communication in the surrounding area

(iii) the tracing of auditory processing on subsequent levels up to a third order interneuron including a switch of excitation to inhibition

(iv) a greater variety of selective responses to ultrasonic stimuli and temporal parameters than described for thoracic neurons

(v) secondary spike activity following stimulation with a latency of ca. $600 \mathrm{~ms}$ and graded potentials exhibiting remarkably long time constants of several hundred milliseconds

Several of these mechanisms lead to a closer correspondence of neuronal activity to species specific behaviour than found in the thorax. 


\section{References}

Alexander RD (1967) Acoustical communication in arthropods. Ann Rev Entomol 12:495-526

Alle H, Geiger JR (2006) Combined analog and action potential coding in hippocampal mossy fibers. Science 311:1290-1293

Alle H, Geiger JR (2008) Analog signalling in mammalian cortical axons. Curr Opin Neurobiol 18:314-320

Antonov I, Antonova I, Kandel ER, Hawkins RD (2003) Activity-dependent presynaptic facilitation and Hebbian LTP are both required and interact during classical conditioning in Aplysia. Neuron 37:135-147

Atkins G, Chiba A, Atkins S, Stout JF (1988) Low-pass filtering of sound signals by a highfrequency brain neuron and its input in the cricket Acheta domestica L. J Comp Physiol A 164:269-276

Atkins G, Ligman S, Burghardt F, Stout JF (1984) Changes in phonotaxis by the female cricket Acheta domesticus L. after killing identified acoustic interneurons. J Comp Physiol A 154:795-804

Atkins G, Pollack GS (1986) Age dependent occurrence of an ascending axon on the omega neuron of the cricket, Teleogryllus oceanieus. J Comp Neurol 243:527-534

Atkins G, Pollack GS (1987a) Correlation between structure, topographic arrangement, and spectral sensitivity of sound sensitive interneurons in crickets. J Comp Neurol 266:389412

Atkins G, Pollack GS (1987b) Response properties of prothoracic, interganglionic, soundactivated interneurons in the cricket Teleogryllus oceanicus. J Comp Physiol A $161: 681-693$

Bailey WJ (2003) Insect duets: underlying mechanisms and their evolution. Physiol Entomol 28:157-174

Bauer M, Helversen O von (1987) Separate localization of sound recognizing and sound producing neural mechanisms in a grasshopper. J Comp Physiol A 161:95-101

Békésy G von (1960) Experiments in Hearing. McGraw-Hill, New York

Borst A, Egelhaaf $\mathrm{M}$ (1992) In vivo imaging of calcium accumulation in fly interneurons as elicited by visual motion stimulation. Proc Natl Acad Sci USA 89:4139-4143

Borst A, Haag J (2002) Neural networks in the cockpit of the fly. J Comp Physiol 188:419437

Boyan GS (1980) Auditory neurones in the brain of the cricket Gryllus bimaculatus (De Geer). J Comp Physiol A 140:81-93

Boyan GS (1981) Two-tone suppression of an identified auditory neuron in the brain of the cricket Gryllus bimaculatus (De Geer). J Comp Physiol A 144:117-125

Bradbury JW, Vehrencamp SL (1998) Principles of animal communication. Sunderland, MA: Sinauer

Brodfuehrer PD, Hoy RR (1990) Ultrasound sensitive neurons in the cricket brain. J Comp Physiol A 166:651-662

Burrows M, Laurent G (1993) Synaptic potentials in the central terminals of locust proprioceptive afferents generated by other afferents from the same organ. J Neurosci 13(2):808-819 
Bush SL, Schul J (2006) Pulse-rate recognition in an insect: evidence of a role for oscillatory neurons. J Comp Physiol A 192:113-121

Byrne JH, Kandel ER (1996) Presynaptic facilitation revisited: State and time dependence. J Neurosci 16:425-435

Cade WH (1984) Effects of fly parasitoids on nightly calling duration in field crickets. Can J Zool 62:226-228

Cattaert D, El Manira A, Clarac F (1994) Chloride conductance process both presynaptic inhibition and antidromic spikes in primary afferent. Brain Res 666:109-112

Cattaert D, El Manira A (1999) Shunting versus inactivation: analysis of presynaptic inhibitory mechanisms in primary afferents of the crayfish. J Neurosci 19:6079-6089

Clarac F, Cattaert D (1996) Invertebrate presynaptic inhibition and motor control. Exp Brain Res 112:163-180

Clark B, Häusser M (2006) Neural coding: hybrid analog and digital signalling in axons. Curr Biol 16:R585-R588

Dobler S, Heller KG, Helversen O von (1994a) Song pattern recognition and an auditory time window in the female bushcricket Ancistrura nigrovittata. J Comp Physiol A 175:67-74

Dobler S, Stumpner A, Heller KG (1994b) Sex-specific spectral tuning for the partner's song in the duetting bushcricket Ancistrura nigrovittata (Orthoptera: Phaneropteridae). J Comp Physiol A 175:303-310

Doherty JA (1991) Song recognition and localisation in phonotaxis behaviour of the field cricket, Gryllus bimaculatus (Orthoptera: Gryllidae). J Comp Physiol 168:213-222

Edwards DH (1990) Mechanisms of depolarizing inhibition at the crayfish giant motor synapse. I. Electrophysiology. J Neurophysiol 64:532-540

Ewing AW (1984) Acoustic signals in insect sexual behaviour. In: Lewis T (ed) Insect Communication. $12^{\text {th }}$ Symposium of the Royal Entomological Society of London. Academic Press, London, pp 223-240

Fielden A (1960): Transmission through the last abdominal ganglion of the dragonfly nymph, Anax imperator. J Exp Biol 37:832-844

Fonseca PJ, Münch D, Hennig RM (2000) Auditory perception: How cicadas interpret acoustic signals. Nature 405:297-298

Forrest TG (1994) From sender to receiver: propagation and environmental effects on acoustic signals. Am Zool 34:644-654

Furshpan EJ, Furukawa T (1962) Intracellular and extracellular responses of the several regions of the Mauthner cell of the goldfish. J Neurophysiol 25:732-771

Fuster JM, Creutzfeldt OD, Straschill M (1965) Intracellular recording of neuronal activity in the visual system. Z Vergl Physiol 49:605-622

Gerhardt HC, Huber F (2002) Acoustic communication in insects and anurans; common problems and diverse solutions. Univ of Chicago Press, Chicago

Gersdorff H von, Schneggenburger R, Weis S, Neher E (1997) Presynaptic Depression at a Calyx Synapse: The Small Contribution of Metabotropic Glutamate Receptors. J Neurosci 17:8137-8146

Gras H, Hörner M, Runge L, Schürmann FW (1990) Prothoracic DUM neurons of the cricket Gryllus bimaculatus respond to natural stimuli activity in walking behaviour. J Comp Physiol A 166:901-914

Harrison RG (1986) Pattern and process in a narrow hybrid zone. Heredity 56:337-349 
Haydon PG, Winlow W (1982) Multipolar neurones of Lymnaea stagnalis. I. Multiple spike initiation sites and propagation failure allow neuronal compartmentalization. J Comp Physiol A 147:503-510

Hedwig B (2000) Control of cricket stridulation by a command neuron: efficacy depends on the behavioral state. J Neurophysiol 83:712-722

Hedwig B (2006) Pulses, patterns and paths: neurobiology of acoustic behaviour in crickets. J Comp Physiol A 192:677-689

Hedwig B, Baden T (2009) Presynaptic processing in auditory afferents of bushcrickets. Poster abstract in Neuroforum Feb 2009 (1) Vol. XV, Supplement, 8th Meeting German Neuroscience Soc., Göttingen, Germany, Mar 25-29

Hedwig B, Knepper M (1992) NEUROLAB, a comprehensive program for the analysis of neurophysiological and behavioural data. J Neurosci Methods 45:135-148

Heinrich R, Jatho M, Kalmring K (1993) Acoustic transmission characteristics of the tympanal tracheae of bushcrickets (Tettigoniidae). II: Comparative studies of the tracheae of seven species. J Acoust Soc Am 93:3481-3489

Heller KG (1990) Evolution of song pattern in east Mediterranean Phaneropterinae: constraints by the communication system. In: Bailey WJ, Rentz DCF (eds) The Tettigoniidae: biology, systematics and evolution. Crawford House, Bathurst, pp 130151

Heller KG, Helversen D von (1986) Acoustic communication in phaneropterid bushcrickets: species-specific delay of female stridulatory response. Behav Ecol Sociobiol 18:189198

Helversen D von, Helversen O von (1983) Species recognition and acoustic localization in acridid grasshoppers: a behavioural approach. In: Huber F, Markl H (eds) Neuroethology and behavioural physiology. Springer Berlin Heidelberg, pp 95-107

Helversen D von, Helversen $O$ von (1995) Acoustic pattern recognition and orientation in orthopteran insects: parallel or serial processing? J Comp Physiol A 177:767-774

Helversen D von, Helversen O von (1998) Acoustic pattern recognition in a grasshopper: processing in the time or frequency domain? Biol Cybernet 79:467-476

Hennig RM (1988) Ascending auditory interneurons in the cricket Teleogryllus commodus (Walker): comparative physiology and direct connections with afferents. J Comp Physiol A 163:135-143

Hennig RM (1989) Neuromuscular activity during stridulation in the cricket Teleogryllus commodus. J Comp Physiol A 165:837-846

Hennig RM (2003) Acoustic feature extraction by cross-correlation in crickets? J Comp Physiol A 189:589-598

Hennig RM, Franz A, Stumpner A (2004) Processing of auditory information in insects. Microsc Res Tech 63:351-374

Hoy RR, Paul RC (1973) Genetic control of song specificity in crickets. Science 180:82-83

Jan LY, Jan YN (1982) Antibodies to horseradish peroxidase as specific neuronal markers in Drosophila and in grasshopper embryos. Proc Natl Acad Sci USA 79:2700-2704

Kennedy D, McVitte J, Calabrese R, Fricke RA, Craelius W, Chiapella P (1980) Inhibition of mechanosensory interneurons in the crayfish. I. Presynaptic inhibition from giant fibers. J Neurophysiol 43:1495-1509

Kandel ER, Siegelbaum SA (2000) Transmitter release. In: Kandel E, Schwartz J, Jessell T (eds) Principles of Neural Science (4th edition). McGraw-Hill, New York, pp 253-279 
Kita H, Armstrong W (1991) A biotin-containing compound $\mathrm{N}$-(2-aminoethyl)biotinamide for intracellular labeling and neuronal tracing studies: Comparison with biocytin. J Neurosci Methods 37:141-150

Kusano K, Landau EM (1975) Depression and recovery of transmission at the squid giant synapse. J Physiol (Lond) 245:13-32

Lang F, Brandt G, Glahe M (1993): A versatile multichannel acoustic stimulator controlled by a personal computer. In: Elsner N, Heisenberg M (eds) Gene-Brain-Behavior. Thieme, Stuttgart, p 892

Levine RB, Murphey RK (1980) Pre- and postsynaptic inhibition of identified giant interneurons in the cricket (Acheta domesticus). J Comp Physiol A 135:269-282

Libersat F, Hoy RR (1991) Ultrasonic startle behavior in bushcrickets (Orthoptera; Tettigoniidae). J Comp Physiol A 169:507-514

Lin Y, Kalmring K, Jatho M, Sickmann T, Rössler W (1993) Auditory receptor organs in the forelegs of Gampsocleis gratiosa (Tettigoniidae): morphology and function of the organs in comparison to the frequency parameters of the conspecific song. J Exp Zool 267:377-388

Machens CK, Schütze H, Franz A, Kolesnikova O, Stemmler MB, Ronacher B, Herz AV (2003) Single auditory neurons rapidly discriminate conspecific communication signals. Nat Neurosci 6:341-342

Mcllwain JT, Creutzfeld OD (1967) Microelectrode study of synaptic excitation and inhibition in the lateral geniculate nucleus of the rat. J Neurophysiol 30:1-21

Mesce KA, Klukas KA, Brelje TC (1993) Improvements for the anatomical characterization of insect neurons in whole mount: The use of cyanine-derived fluorophores and laser scanning confocal microscopy. Cell Tissue Res 271:381-397

Michelsen A (1971) The physiology of the locust ear. I. Frequency sensitivity of single cells in the isolated ear, II. Frequency discrimination based upon resonances in the tympanum, and III. Acoustical properties of the intact ear. Z Vgl Physiol 71:49-128

Michelsen A (1978) Sound reception in different environments. In: Ali MA (ed) Sensory ecology. Plenum, New York, pp 345-372

Michelsen A, Heller KG, Stumpner A, Rohrseitz K (1994) A new biophysical method to determine the gain of the acoustic trachea in bushcrickets. J Comp Physiol A 175:145151

Moiseff A, Pollack GS, Hoy RR (1978) Steering responses of flying crickets to sound and ultrasound. Proc Natl Acad Sci USA 75:4052-4056

Molina J, Stumpner A (2005) Effects of pharmacological treatment and photoinactivation on the directional responses of an insect neuron. J Exp Zoolog A 303(12):1085-1103

Mörchen A, Rheinlaender J, Schwartzkopff J (1978) Latency shift in insect auditory-nerve fibers: neuronal time cue of sound direction. Naturwissenschaften 65:656-657

Müller M, Homberg U, Kühn A (1997) Neuroarchitecture of the lower division of the central body in the brain of the locust (Schistocerca gregaria). Cell Tissue Res 288:159-176

Nabatiyan A, Poulet JFA, de Polavieja GG, Hedwig B (2003) Temporal pattern recognition based on instantaneous spike rate coding in a simple auditory system. J Neurophysiol 90:2484-2493

Nickle AD (1976) Interspecific differences in frequency and other physical parameters of pair forming sounds of bush katydids (Orthoptera: Tettigoniidae: Phaneropterinae). Ann Entomol Soc Am 69:1136-1144 
Nicolls J, Wallace BG (1978) Modulation of transmission at an inhibitory synapse in the central nervous system of the leech. J Physiol 281:157-170

Nolen TG, Hoy RR (1986) Phonotaxis in flying crickets. I. Attraction to the calling song and avoidance of bat-like ultrasound are discrete behaviors. J Comp Physiol A 159:423-439

Oldfield BP (1982) Tonotopic organisation of auditory receptors in Tettigoniidae (Orthoptera: Ensifera). J Comp Physiol A 147:461-469

Oldfield BP (1983) Central projections of primary auditory fibers in Tettigoniidae (Orthoptera: Ensifera). J Comp Physiol A 151:389-395

Oldfield BP, Kleindienst H, Huber F (1986) Physiology and tonotopic organization of auditory receptors in the cricket Gryllus bimaculatus DeGreer. J Comp Physiol A 159:457-464

Oshinsky ML, Hoy RR (2002) Physiology of auditory afferents in an acoustic parasitoid fly. J Neurosci 22:7254-7263

Otte D (1992) Evolution of cricket songs. J Orthoptera Res 1:25-49

Palmer LM, Stuart GJ (2006) Site of action potential initiation in layer 5 pyramidal neurons. J Neurosci 26:1854-1863

Pires A, Hoy RR (1992) Temperature coupling in cricket acoustic communication: I. Field and laboratory studies of temperature effects on calling song production and recognition in Gryllus firmus. J Comp Physiol A 171:69-78

Pollack GS, Hoy R (1979) Temporal pattern as a cue for species specific calling song recognition in crickets. Science 204:429-432

Pollack G (2000) Who, what, where? Recognition and localization of acoustic signals by insects. Curr Opin Neurobiol 10:763-767

Popov AV, Markovich AM, Andjan AS (1978) Auditory interneurones in the prothoracic ganglion of the cricket, Gryllus bimaculatus. I. The large segmental auditory neuron (LSAN). J Comp Physiol A 126:183-192

Poulet JF, Hedwig B (2006) The cellular basis of a corollary discharge. Science 311:518-522

Purves D, Sakmann B (1974) The effect of contractile activity on fibrillation and extrajunctional acetylcholine-sensitivity in rat muscle maintained in organ culture. J Physiol (Lond) 237:157-182

Ragge DR, Reynolds WJ (1988) The songs and taxonomy of the grasshoppers of the Chorthippus biguttulus group in the Iberian Peninsula (Orthoptera: Acrididae). J Nat Hist 22:897-929

Rheinlaender J (1975) Transmission of Acoustic Information at Three Neuronal Levels in the Auditory System of Decticus verrucivorus (Tettigoniidae, Orthoptera). J comp Physiol A 97:1-53

Reiss RF (1964) A theory of resonance. In: Reiss RF (ed) Neural theory and modeling. Stanford UP, Stanford, pp 105-137

Rheinlaender J, Mörchen A (1979) Time-intensity trading in locust auditory interneurones. Nature 281:672-674

Robbins J, van der Kloot WG (1958) The effect of picrotoxin on peripheral inhibition in the crayfish. J Physiol (Lond) 143:541-552

Robles L, Ruggero MA (2001) Mechanics of the mammalian cochlea. Physiol Rev 81:13051352

Römer H (1983) Tonotopic organization of the auditory neuropile in the bushcricket Tettigonia viridissima. Nature 306:60-62 
Römer H (1987) Representation of auditory distance within a central neuropile of the bushcricket Mygalopsis marki. J Comp Physiol A 161:33-42

Römer H, Krusch M (2000) A gain-control mechanism for processing of chorus sounds in the afferent auditory pathway of the bushcricket Tettigonia viridissima (Orthoptera; Tettigoniidae). J Comp Physiol A 186:181-191

Römer H, Marquart V, Hardt M (1988) Organization of a sensory neuropile in the auditory pathway of two groups of Orthoptera. J Comp Neurol 275:201-215

Römer H, Seikowski U (1985) Responses to model songs of auditory neurons in the thoracic ganglia and brain of the locust. J Comp Physiol A 156:845-860

Ronacher B, Helversen D von, Helversen O von (1986) Routes and stations in the processing of auditory directional information in the CNS of a grasshopper, as revealed by surgical experiments. J Comp Physiol A 158:363-374

Rudomin P, Schmidt RF (1999) Pre-synaptic inhibition in the vertebrate spinal cord revisited. Exp Brain Res 129:1-37

Ryder J, Siva-Jothy MT (2000) Male calling song provides a reliable signal of immune function in a cricket. Proc R Soc Lond B 263:1171-1175

Sakaluk SK, Belwood JJ (1984) Gecko phonotaxis to cricket calling song: a case of satellite predation. Anim Behav 32:659-662

Schildberger K (1984a) Temporal selectivity of identified auditory neurons in the cricket's brain. J Comp Physiol A 155:171-185

Schildberger K (1984b) Multimodal interneurons in the cricket brain: properties of identified extrinsic mushroom body cells. J Comp Physiol A 154:71-79

Schildberger K, Hörner M (1988) The function of auditory neurons in cricket phonotaxis. I. Infuence of hyperpolarization of identifed neurons on sound localization. J Comp Physiol A 163:621-631

Schul J (1997) Neuronal basis of phonotactic behaviour in Tettigonia viridissima: processing of behaviourally relevant signals by auditory afferents and thoracic interneurons. J Comp Physiol A 180:573-583

Schul J (1998) Song recognition by temporal cues in a group of closely related bushcricket species (genus Tettigonia). J Comp Physiol A 183:401-410

Schwabe J (1906) Beiträge zur Morphologie und Histologie der tympanalen Sinnesapparate der Orthopteren. Zoologica Stuttgart 50:1-154

Selverston AI, Kleindienst HU, Huber F (1985) Synaptic connectivity between cricket auditory interneurons as studied by selective photoinactivation. J Neurosci 5:1283-1292

Shapiro E, Castellucci VF, Kandel ER (1980) Presynaptic membrane potential affects transmitter release in an identified neuron in Aplysia by modulating the $\mathrm{Ca}^{2+}$ and $\mathrm{K}^{+}$ currents. Proc Natl Acad Sci USA 77:629-633

Shu Y, Hasenstaub A, Duque A, Yu Y, McCormick DA (2006) Modulation of intracortical synaptic potentials by presynaptic somatic membrane potential. Nature 441:761-765

Siegelbaum SA, Koester J (2000) lon channels. In: Kandel E, Schwartz J, Jessell T (eds) Principles of Neural Science (4th edition). McGraw-Hill, New York, pp 105-124

Sickmann T (1996) Vergleichende funktionelle und anatomische Untersuchungen zum Aufbau der Hör- und Vibrationsbahn im thorakalen Bauchmark von Laubheuschrecken. Cuvillier Verlag, Göttingen 
Simmons LW (1988) The calling song of the field cricket, Gryllus bimaculatus (De Geer): constraints on transmission and its role in intermale competition and female choice. Anim Behav 36:380-394

Simmons JA (1989) A view of the world through the bat's ear: the formation of acoustic images in echolocation. Cognition 33:155-199

Spencer WA, Kandel ER (1961) Electrophysiology of hippocampal neurons. IV. Fast prepotentials. J Neurophysiol 24:272-285

Stabel J, Wendler G, Scharstein H (1989) Cricket phonotaxis: localization depends on recognition of the calling song pattern. J Comp Physiol A 165:165-177

Stölting H, Stumpner A (1998) Tonotopic organization of auditory receptors in the bushcricket Pholidoptera griseoaptera (Tettigoniidae, Decticini). Cell Tissue Res 294:377-386

Stritih N, Stumpner A (2009) Vibratory interneurons of the non-hearing cave cricket indicate evolutionary origin of sound processing elements in Ensifera. Zoology 112:48-68

Stumpner A (1995) Some new local and descending auditory neurons in the prothoracic ganglion of crickets and bushcrickets. In: Elsner N, Menzel R (eds) Göttingen neurobiology report 1995. Thieme, Stuttgart, pp A274

Stumpner A (1996) Tonotopic organization of the hearing organ in a bushcricket. Physiological characterization and complete staining of auditory receptor cells. Naturwissenschaften 83:81-84

Stumpner A (1997) An auditory interneurone tuned to the male song frequency in the duetting bushcricket Ancistrura nigrovittata (Orthoptera, Phaneropteridae). J Exp Biol 200:1089-1101

Stumpner A (1998) Picrotoxin eliminates frequency selectivity of an auditory interneuron in a bushcricket. J Neurophysiol 79:2408-2415

Stumpner A (1999a) Comparison of morphology and physiology of two plurisegmental sound-activated interneurones in a bushcricket. J Comp Physiol A 185:199-205

Stumpner A (1999b) An interneurone of unusual morphology is tuned to the female song frequency in the bushcricket Ancistrura nigrovittata (Orthoptera, Phaneropteridae). J Exp Biol 202:2071-2081

Stumpner A (2001) Sound activated neurones and their potential function in a bushcricket - a synopsis. In: Elsner N, Kreutzberg GW (eds) Göttingen Neurobiology report 2001. Thieme, Stuttgart, pp A370

Stumpner A (2002) A species-specific frequency filter through specific inhibition, not specific excitation. J Comp Physiol A 188:239-248

Stumpner A, Atkins G, Stout JF (1995) Processing of unilateral and bilateral auditory inputs by the ON1 and L1 interneurons of the cricket Acheta domesticus and comparison to other cricket species. J Comp Physiol A 177:379-388

Stumpner A, Helversen D von (2001) Evolution and function of auditory systems in insects. Naturwissenschaften 88:159-170

Stumpner A, Meyer S (2001) Songs and the function of song elements in four duetting bushcricket species (Ensifera, Phaneropteridae, Barbitistes). J Insect Behav 14:511534

Stumpner A, Molina J (2006) Diversity of intersegmental auditory neurons in a bush cricket. J Comp Physiol A 192:1359-1376

Surlykke A (1984) Hearing in notodontid moths: a tympanic organ with a single auditory neurone. J Exp Biol 113:323-335 
Tauber E, Cohen D, Greenfield MD, Pener MP (2001) Duet singing and female choice in the bushcricket Phaneroptera nana. Behav 138:411-430

Tauc L (1962) Identification of active membrane areas in the giant neuron of Aplysia. J Gen Physzol 45:1099-1115

Tessier-Lavigne M (2000) Visual processing by the retina. In: Kandel E, Schwartz J, Jessell T (eds). Principles of Neural Science (4th edition). McGraw-Hill, New York, pp 507-522

Triblehorn JD, Schul J (2009) Sensory-Encoding Differences Contribute to Species-Specific Call Recognition Mechanisms. J Neurophysiol 102:1348-1357

Vedenina VYu \& Helversen O von (2003) Complex courtship in a bimodal grasshopper hybrid zone. Behav Ecol Sociobiol 54:44-54

Watson ADH, Hardt M (1996) Distribution of synapses on two local auditory interneurons ON1 and ON2 in the prothoracic ganglion of the cricket: relationships with GABAimmunoreactive neurons. Cell Tissue Res 283:231-246

Weber T, Thorson J (1989) Phonotactic behavior of walking crickets. In: Huber F, Moore TE, Loher W (eds) Cricket behavior and neurobiology. Cornell University Press, Ithaca, pp 310-339

Wiese K, Eilts K (1985) Evidence for matched frequency dependence of bilateral inhibition in the auditory pathway of Gryllus bimaculatus. Zool Jb Physiol 89:181-201

Wilson WD, Tanious FA, Barton HJ, Jones RL, Fox K, Wydra RL, Strekowski L (1990) DNA sequence dependent binding modes of 4',6-diamidino-2-phenylindole (DAPI). Biochem 29:8452-8461

Wohlers DW, Huber F (1978) Intracellular recording and staining of cricket auditory interneurons (Gryllus campestris L., Gryllus bimaculatus De Geer). J Comp Physiol A 127:11-28

Wohlers DW, Huber F (1982) Processing of sound signals by six types of neurons in the prothoracic ganglion of the cricket, Gryllus campestris L. J Comp Physiol A 146:161173

Wohlers DW, Huber F (1985) Topographical organization of the auditory pathway within the prothoracic ganglion of the cricket Gryllus campestris L. Cell Tissue Res 239:555-565

Wollner DA, Catterall WA (1986) Localization of sodium channels in axon hillocks and initial segments of retinal ganglion cells. Proc Natl Acad Sci USA 83:8424-8428

Zhantiev RD, Korsunovskaya OS (1983) Structure and functions of two auditory neurons in the bush cricket Tettigonia cantans Fuess. (Orthoptera, Tettigoniidae). Revue d'Entomol L'URSS 62:462-469

Zimmermann U, Rheinlaender J, Robinson D (1989) Cues for male phonotaxis in the duetting bushcricket Leptophyes punctatissima. J Comp Physiol A 164:621-628 


\section{Appendix}

\section{Solutions used in the experiment}

Phosphate buffer saline (PBS)

$11.5 \mathrm{~g} \mathrm{Na}_{2} \mathrm{HPO}_{4}$

$2.96 \mathrm{~g} \mathrm{NaH}_{2} \mathrm{PO}_{4} \times 2 \mathrm{H}_{2} \mathrm{O}$

$5.84 \mathrm{~g} \mathrm{NaCl}$

Dissolved in 1 litre distilled $\mathrm{H}_{2} \mathrm{O}$ and adjusted to $\mathrm{pH}$ 7.4.

\section{Phosphate buffer saline-tween (PBST)}

\section{$11.5 \mathrm{~g} \mathrm{Na}_{2} \mathrm{HPO}_{4}$}

$2.96 \mathrm{~g} \mathrm{NaH}_{2} \mathrm{PO}_{4} \times 2 \mathrm{H}_{2} \mathrm{O}$

$5.84 \mathrm{~g} \mathrm{NaCl}$

Dissolved in 1 litre distilled $\mathrm{H}_{2} \mathrm{O}$ and adjusted to $\mathrm{pH}$ 7.4. Subsequently, $1 \mathrm{~mL}$ Tween 80 was added and stored at $4^{\circ} \mathrm{C}$.

\section{Saline solution (Fielden 1960)}

$1.5 \mathrm{~g} \mathrm{NaCl}$

$0.02 \mathrm{~g} \mathrm{KCl}$

$0.04 \mathrm{~g} \mathrm{CaCl}_{2}$

$0.04 \mathrm{~g} \mathrm{NaHCO}_{3}$

$0.18 \mathrm{~g}$ TES (N-tris[hidroxymethyl]methyl-2-aminoethanesulfonic acid)

Dissolved in $200 \mathrm{~mL}$ distilled $\mathrm{H}_{2} \mathrm{O}$ and adjusted to $\mathrm{pH}$ 6.8. The saline solution was then stored at $4^{\circ} \mathrm{C}$. 


\section{Neurobiotin detection}

Procedure for the neurobiotin detection (modified after Mesce et al. 1993)

1. Fixation of dissected brains in $4 \%$ paraformaldehyde dissolved in phosphate buffer saline (PBS) for 2 hours at $4{ }^{\circ} \mathrm{C}$

2. Stepwise dehydration in ethanol $(70 \%, 90 \%, 96 \%$ and $100 \%)$ for $10 \mathrm{~min}$. each

3. Permeabilisation with Xylol (Merck) for 5 min.

4. Stepwise rehydration in ethanol $(100 \%, 96 \%, 90 \%$ and $70 \%)$ for $10 \mathrm{~min}$. each

5. Washing two times with PBS for 10 min. each

6. Incubation of the brain in a solution of $1 \mathrm{mg}$ collagenase and $1 \mathrm{mg}$ hyaluronidase per $1 \mathrm{~mL}$ PBS at $37^{\circ} \mathrm{C}$ for 1 hour

7. Washing two times with PBS for 15 min. each

8. Washing three times with phosphate buffer saline-tween (PBST) for $15 \mathrm{~min}$. each

9. Incubation in streptavidin-Cy3 (Rockland Immunochemicals) or fluorescein streptavidin (FITC, Vector laboratories) (1:200 in PBST) at room temperature over night

10. Washing three times with PBST for $15 \mathrm{~min}$. each

11. Washing three times with PBS for 15 min. each

12. Stepwise dehydration in ethanol $(70 \%, 90 \%, 96 \%$ and $100 \%)$ for $10 \mathrm{~min}$. each 


\section{List of Abbreviations}

$\begin{array}{ll}\text { C } & \text { degree Celsius } \\ \text { ca. } & \text { circa } \\ \text { CB } & \text { central body } \\ \text { CDI } & \text { corollary discharge interneuron } \\ \text { cm } & \text { centimetre } \\ \text { CNS } & \text { central nervous system } \\ \text { dB } & \text { decibel } \\ \text { dur. } & \text { duration } \\ \text { e.g. } & \text { for example } \\ \text { EPSP } & \text { excitatory postsynaptic potential } \\ \text { et al. } & \text { et alteri } \\ \text { g } & \text { gram } \\ \text { IPSP } & \text { inhibitory postsynaptic potential } \\ \text { KHz } & \text { kilohertz } \\ \text { min. } & \text { minute } \\ \text { MHz } & \text { megahertz } \\ \text { mL } & \text { millilitre } \\ \text { mm } & \text { millimetre } \\ \text { ms } & \text { millisecond } \\ \text { M } \Omega & \text { mega ohm } \\ \mathrm{N} & \text { number of specimen } \\ \mathrm{n} & \text { number of single values } \\ \text { nA } & \text { nano ampere } \\ \text { PAD } & \text { primary afferent depolarisation } \\ \mathrm{PBS} & \text { phosphate buffer saline } \\ \mathrm{PBST} & \text { phosphate buffer saline-tween } \\ \mathrm{PTX} & \text { picrotoxin } \\ \mathrm{SPL} & \text { sound pressure level } \\ \mathrm{SD} & \text { standard deviation } \\ \mathrm{SE} & \text { standard error } \\ \mu \mathrm{g} & \text { microgram } \\ \mu \mathrm{m} & \text { micrometre } \\ & \end{array}$




\section{Acknowledgements}

Most of all I like to thank Prof. Dr. Andreas Stumpner for giving me the opportunity to work in his laboratory and to write this doctoral thesis under his supervision. You awaked my interest in the neurobiology of insect hearing and strengthened it ever since. Thank you for lending me your ear anytime I had problems.

Furthermore, I owe many thanks to my other thesis committee members. Prof. Dr. Norbert Elsner I would like to thank for his support during my doctoral thesis and the wonderful trips to mount Tomaros and the hybrid eldorado. Thanks also go to Prof. Dr. Tobias Moser for his helpful suggestions on my subject at the thesis committee meetings.

Additionally, I like to thank the members of my defense committee Prof. Dr. R. Heinrich, Prof. Dr. M. Göpfert and Prof. Dr. A. Fiala for their interest in my doctoral thesis.

I am very thankful to Marianne Fischer of the "Marianne \& Dr. Fritz Walter FischerFoundation", who financed most of this work. Furthermore, I would like to thank the GGNB Sensory and Motor Neuroscience program for providing me a Bridging Fund to finish this doctoral thesis.

Many thanks go to Matthias Schink for his immense help with the animal care, to Patricia Sprysch and Silvia Gubert for their patients with the unborn, and Margret Winkler for the subsequent slicing work.

I am very grateful to Dr. Heribert Gras and Andrea Wirmer for their valuable help with nebulous statistics.

I also like to thank all my colleagues of the neurobiology and cell biology department for the familiar atmosphere at the numerous "lab meetings" with coffee and cake.

I am very grateful to my family for their continuous faith in my work and their encouraging words when I had hard times with the tiny nerve cells.

Finally, I like to thank the most important person in my life - Dani. We have some exciting times ahead of us and I am really lucky to start the new life with you as one family. 


\section{Publications}

\section{Articles in peer-reviewed journals:}

Ostrowski TD, Stumpner A (submitted) Frequency processing on subsequent auditory levels of a bush cricket.

Ostrowski TD, Sradnick J, Stumpner A, Elsner N (2009) The elaborate courtship behavior of Stenobothrus clavatus Willemse, 1979 (Acrididae: Gomphocerinae). J Orthop Res 18(2): 171-182

Heller KG, Ostrowski TD, Hemp C (in press) Singing and hearing in Aerotegmina kilimandjarica (Tettigoniidae: Hexacentrinae), a species with unusual low carrier frequency of the calling song. Bioacoustics 19(2)

\section{Poster presentations:}

Ostrowski TD, Stumpner A (2009) An uncommon gain control - amplification instead of inhibition. $32^{\text {nd }}$ Göttingen Neurobiology Conference $-8^{\text {th }}$ Meeting of the German Neuroscience Society in Göttingen, Germany

Ostrowski TD, Stumpner A (2007) Filtering of species specific song parameters via interneurons in a bush cricket's brain. $8^{\text {th }}$ International Congress in Vancouver, Canada

Ostrowski TD, Stumpner A (2007) Filtering of species specific song parameters via interneurons in a bush cricket's brain. Kleinsthirnkonferenz in Tutzingen, Germany

Ostrowski TD, Stumpner A (2007) Adaptation of auditory neurons in a bushcricket. $31^{\text {st }}$ Göttingen Neurobiology Conference $-7^{\text {th }}$ Meeting of the German Neuroscience Society in Göttingen, Germany 


\section{Curriculum Vitae}

Tim Daniel Ostrowski

Mauerstraße 21

37073 Göttingen

Phone: $\quad+49(0) 551-9961163$

E-mail: tostrow@gwdg.de

Date of birth: June $25^{\text {th }}, 1980$

Place of birth: Leer (Loga)

Marital status: unmarried

\section{Education}

$1986-1990$

$1990-1992$

$1992-1996$

$1996-1997$

$1997-2001$

2001
Elementary school Grünenplan

Intermediate school Duingen

Secondary school (Gymnasium) Alfeld/Leine

School year abroad in Lincoln, NE, USA

Secondary school (Gymnasium) Alfeld/Leine

Degree: general qualification for university entrance

\section{Academic studies}

$2001-2006$

Georg-August-University Göttingen

Subject: Biology

2006

Degree: Diploma in biology
Major subject:
Zoology
1. Minor subject: Microbiology
2. Minor subject: Psychology

Diploma thesis: "Adaptation of auditory neurons in a bush cricket"

\section{Conferral of a doctorate}

Since Feb. 2007

Georg-August-University Göttingen

GGNB (Sensory \& Motor Neuroscience - since 2008)

Institute for Zoology, Department of Neurobiology

(Supervisor: Prof. Dr. Andreas Stumpner)

Doctoral thesis: "Filtering of species specific song parameters via interneurons in a bush cricket's brain" 


\section{Stipends}

Feb. 2007 - Aug. 2009 Doctoral-scholarship of the Marianne and Dr. Fritz Walter Fischer-Foundation

Since Sept. $2009 \quad$ Bridging Fund of the Göttingen Graduate School for Neurosciences and Molecular Biosciences (GGNB)

\section{Part-time work \& research/teaching experience}

Sept. 2002 - Oct. 2009 Part-time work: Night care in a house for mentally disabled adolescents, Ifas GmbH Göttingen

May - June $2004 \quad$ Lab course: Neuroanatomy/-physiology of the hearing system in grasshoppers, University of Göttingen

Mar. - Oct. 2005 Student assistant at the University Göttingen

Teaching: first mandatory internship in Zoology: Morphology, functionality and basics of behaviour Research: Analysis of hybrids of two grasshopper species

July - Sept. 2005 \&

Field project in Greece

June - Aug. 2006

Subject: "Ethology and Geography of hybrids of two closely related grasshopper species"

June 2006 - Jan. 2007 Graduate assisted at the University Göttingen

Research: Analysis of hybrids of two grasshopper species

Feb. - Mar. 2007

Graduate assisted at the University Göttingen

Teaching: Advanced neurophysiology course

July 2008

GGNB Method course

Oct. - Nov. 2008

Teaching: "Insect audition 1"

Advanced practicals at the University Göttingen

Teaching: "Neuroscience and behaviour"

Date:

Signature 